CE MÉMOIRE A ÉTÉ RÉALISÉ À

L'UNIVERSITÉ DU QUÉBEC EN ABITIBI-TÉMISCAMINGUE

dANS le CADRE DU PROGRAMME de LA maîtRISE

EN GESTION DES PETITES ET MOYENNES ORGANISATIONS

EXTENSIONNÉ DE L'UNIVERSITÉ DU QUÉBEC

À CHICOUTIMI 


\section{Bibliothèque}

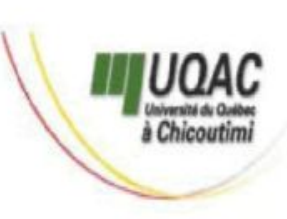

\section{Mise en garde/Advice}

Afin de rendre accessible au plus Motivated by a desire to make the grand nombre le résultat des results of its graduate students' travaux de recherche menés par ses research accessible to all, and in étudiants gradués et dans l'esprit des accordance with the rules règles qui régissent le dépôt et la governing the acceptation and diffusion des mémoires et thèses diffusion of dissertations and produits dans cette Institution, theses in this Institution, the I'Université du Québec à Université du Québec à Chicoutimi (UQAC) est fière de Chicoutimi (UQAC) is proud to rendre accessible une version make a complete version of this complète et gratuite de cette œuvre. work available at no cost to the reader.

L'auteur conserve néanmoins la The author retains ownership of the propriété du droit d'auteur qui copyright of this dissertation or protège ce mémoire ou cette thèse. thesis. Neither the dissertation or Ni le mémoire ou la thèse ni des thesis, nor substantial extracts from extraits substantiels de ceux-ci ne it, may be printed or otherwise peuvent être imprimés ou autrement reproduced without the author's reproduits sans son autorisation. permission. 
Universitê du Qứbec

en Abitibi-Témiscamingue

\section{Volume I}

REFLEXION METHODOLOGIQUE

RELATIVE A L'ETUDE DES BESOINS

D' INFORMATION DES ENTREPRISES

DU GROUPE HAREL, DROUIN

Par

Suzy Paucher

B.A.A. Adm.A.

Novembre 1990 
"Ce mémoire a été réalisé à l'Université du Québec en Abitibi-Témiscamingue dans le cadre du programme de mâtrise en gestion des petites et moyennes organisations extensionné de l'Université du Québec à Chicoutimi à l'Université du Québec en Abitibi-Témiscamingue." 
Résumé

Description, analyse et developpement d'un systeme d'information comptable pour les entreprises du groupe Harel, Drouin

Le mémoire de recherche-action mentionné ci-dessus est présenté en deux volumes. Le premier vous présente la méthodologie employée pour réaliser 1'étude. Le second vous décrit le système d'information comptable des entreprises du groupe Harel, Drouin. Dans ce volume, nous analysons le système et développons un système d'information répondant aux besoins identifiés au cours de la recherche.

Cette recherche-action a été réalisée avec la méthodologie de Davis et les diagrammes de flux de données développés par Gane et Sarson. La méthodologie de Davis nous permet de déterminer le risque associé au développement d'un système d'information. Le risque associé au développement du système d'information comptable des entreprises du groupe Harel, Drouin est moyennement faible. Dans ce cas, Davis nous propose d'utiliser la méthodologie basée sur l'analyse des caractéristiques du système opérant. L'analyse de ces caractéristiques a été réalisée à l'aide de $1 \mathrm{a}$ méthode de 1 'analyse des entrées-traitementssorties. Nous avons utilisé les diagrammes de Gane et Sarson pour faire cette analyse.

La recherche-action est complétée par une réflexion sur l'utilisation de cette méthodologie dans les petites et moyennes entreprises.

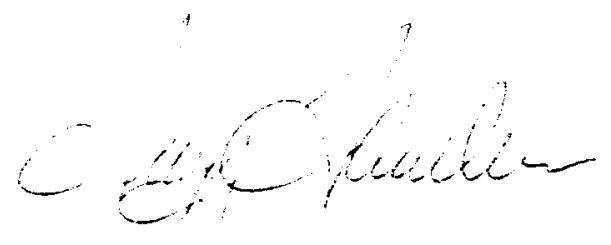

Suzy Faucher

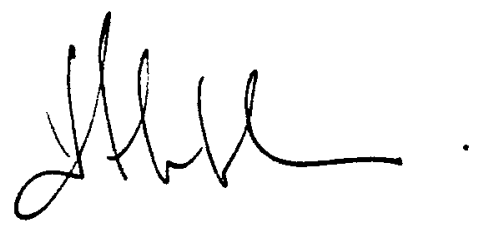

Monsieur Louis A. Lefebvre Ph.d. Directeur de recherche 


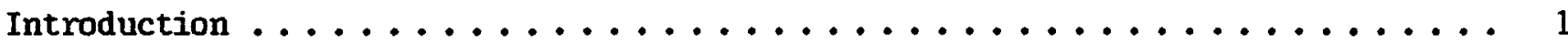

1. Définition des termes utilisés $\ldots \ldots \ldots \ldots \ldots \ldots$

1.1 Système d'information et système d'information comptable . . . . . . . 3

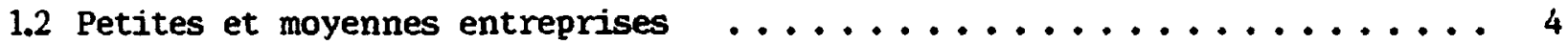

2. Définition de la recherche-action ..................... 5

3. Méthodologie employée pour effectuer la recherche-action $\ldots \ldots \ldots$. . . . 7

3.1 Evaluation de l'incertitude et sélection d'une stratégie $\ldots \ldots$. . . . . 8

3.1.1 Evaluation de $l^{\prime}$ incertitude globale du projet $\ldots \ldots \ldots$....... 8

3.1.2 Stratégie employée selon l'incertitude globale du projet . . . . . . 11

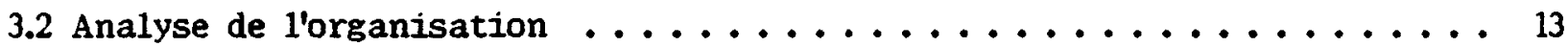

3.2.1 Présentation de l'organisation ....................... 13

3.2.2 Présentation du système d'information comptable (système objet) des entreprises du groupe Harel, Drouin ................ 14

3.3 Evaluation de l'incertitude globale du processus d'analyse des besoins d'information des entreprises du Groupe Harel, Drouin ........... 15

3.4 Stratégie et méthodologie employé . . . . . . . . . . . . . . . . . 16

3.5 Description de la méthodologie des diagrammes de flux d'information . . 18

3.6 Description de la méthodologie utilisée pour réaliser l'étude . . . . . . 20

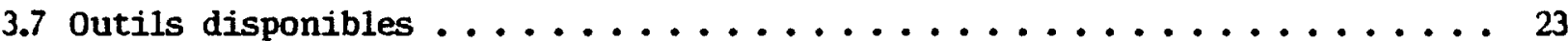

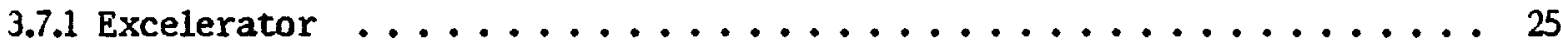

3.7 .2 Logiciel Consoi-MCD . . . . . . . . . . . . . . . . . . . . 25

3.7.3 Logiciel Easyflow . . . . . . . . . . . . . . . . . . 26 
3.7.4 Logiciel Silverrun - DFD ...................... 27

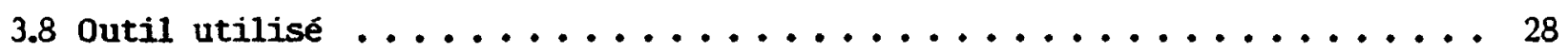

4. Connaissances à tirer du point de vue méthodologique, théorique et conceptuel de l'expérience vécue . . . . . . . . . . . . . . . . . . . . . . 29

4.1 Utilisation de la méthodologie chez les entreprises du groupe Harel

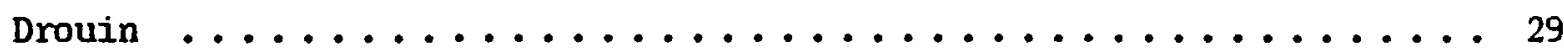

4.1 .1 Etude du système existant ............................ 30

4.1.2 Analyse des caractéristiques du systẻme opérant . . . . . . . . 31

4.1.3 Amélioration de la méthodologie .................... 31

4.2 Autres éléments à ajouter à la méthodologie ............... 32

4.2 .1 Notion de propriétaires de système .................. 32

4.2 .2 Notion d'acteurs (ou utilisateurs) du système . . . . . . . . . . 33

4.2.3 Applicabilité de la méthode dans la petite entreprise . . . . . . . 34

4.2.4 Applicabilité de la méthode dans la moyenne entreprise . . . . . . 35

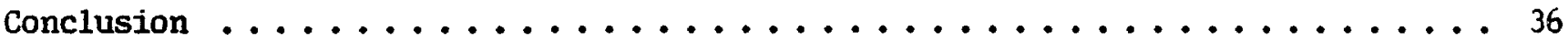

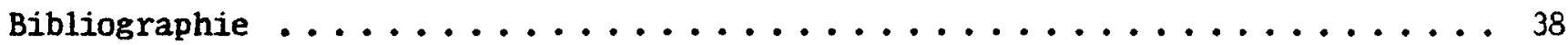




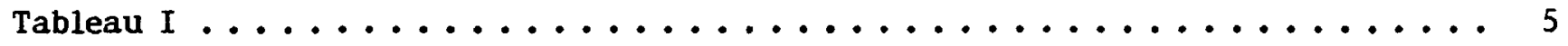

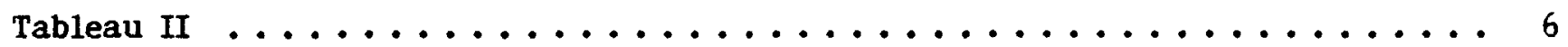

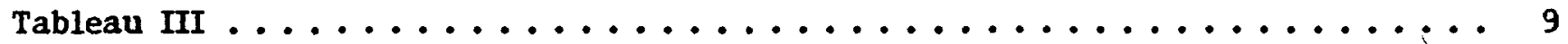

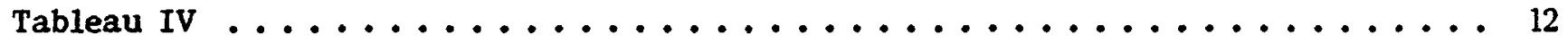

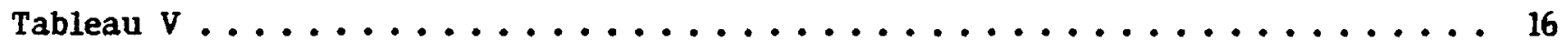

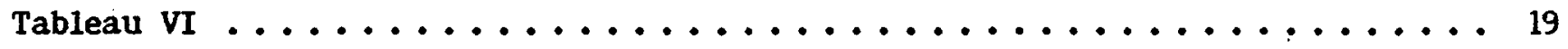

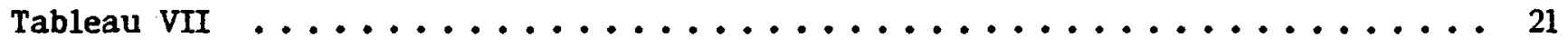




\section{Introduction}

Le mandat initial consistait à effectuer une recherche-action dans le but:

- d'identifier les besoins d'information auxquels le système d'information des entreprises du groupe Harel, Drouin ne répond pas

- déterminer les changements à effectuer au système afin qu'il réponde en tout ou en partie aux besoins identifiés.

- mettre en place un plan de développement rationnel de la gestion de l'information pour les trois prochaines années.

- proposer et mettre en place une structure et des procédés permettant d'assurer une gestion saine et efficace de la micro-informatique.

Le mandat avait été négocié sans qu'aucune étude préliminaire n'ait été effectuée. Afin de procéder à la recherche demandée, nous avons identifié les principales activités ( les sous-systèmes) composant le système d'information. Lors de l'identification des activités, nous avons constaté:

- que toutes les données relatives au système comptable étaient traitées à l'extérieur,

- que ce traitement engendrait des délais et couts importants,

- qu'il obligeait l'entreprise à effectuer des tâches qui auraient pu être évitées si un autre mode de traitement était utilisé,

- que le système informatique employé pour traiter les données était vieux et commençait à présenter des signes de fatigue. 
Les éléments précédant ont fait l'objet de discussions avec les dirigeants de l'entreprise. Lors de ces discussions, il a été convenu qu'une priorité était accordée au sous-système d'information comptable ${ }^{(1)}$ et aux sous-systèmes qui y sont reliés. donc le considérer comme un système à part entière. 
1. Définition des termes utilisés

Afin de préciser l'objet et le champ de notre recherche, nous devons préciser le sens dans lequel sont employés les termes:

- système d'information et système d'information comptable

- petites et moyennes entreprises

Ces définitions déterminent le cadre de notre recherche et de notre réflexion méthodologique.

1.1 Système d'information et système d'information comptable

Armand St-Pierre définit un système d'information comme étant "1a transformation de données brutes en des rapports utiles à la prise de décision interne et utiles également aux utilisateurs externes" (1).

Jonh G.Burch Jr. mentionne qu'une organisation peut être comme un système total composé de trois sous-systèmes nommés système d'opération, système de management et système d'information. Il définit le sous-système d'information en mentionnant :

The information subsystem is an assemblage or collection of people, machines, ideas, and activities that gather and process data in a manner that will meet the formal information requirements of an organization. Its purpose is to satisfy information requirements including accounting and routine operational needs; planning, controlling, and decision-making needs of all levels of management; and the needs of concerned parties external to the organization. (2)

Suite aux énoncés des deux auteurs, nous définissons un système d'information comme étant un ensemble d'activités réalisées par des individus, manuellement, mécanographiquement ou électroniquement, dans le but de transformer des données pour

1- ST-PIERRE, Armand, Les systèmes d'information comptables et de gestion, Une orientation informatique, Canada, Lidec inc., 1985, page 11.

2- BURCH, John G. Jr. et al., Information Systems: Theory and Pratice, U.S.A., Jonh Wiley \& Sons, Inc., Second Edition, 1979, page 27. 
obtenir de l'information utilisée par les gestionnaires de l'entreprise ou par des utilisateurs externes.

Nous définissons un système d'information comptable comme étant un sous-système du système d'information composé d'un ensemble d'activités réalisées par des individus, manuellement ou mécanographiquement à l'aide de machine comptable, ou électroniquement à l'aide d'un ordinateur, dans le but de transformer des données comptables pour obtenir de l'information financière utilisée par les gestionnaires de l'entreprise ou par des utilisateurs externes.

\subsection{Petites et moyennes entreprises}

Notre revue de littérature nous a permis de constater que les auteurs définissaient les petites et moyennes entreprises en fonction d'un des éléments suivants:

- du nombre d'employés

- de 1'actif lorsqu'il s'agit d'entreprises manufacturières; du chiffre d'affaire lorsqu'il s'agit des autres secteurs d'activité.

- du nombre d'employés et de l'actif lorsqu'il s'agit du secteur manufacturier; du nombre d'employés et du chiffre d'affaire lorsqu'il s'agit des autres secteurs d'activité.

Nous désirons attirer votre attention sur le fait que les éléments mentionnés précédemment nous permettent d'inclure dans le terme "entreprise" toutes les entreprises du secteur public et para-public, et toutes les coopératives. Le nombre d'employés et la valeur des actifs ou le chiffre d'affaire nous permettent d'avoir une idée de l'ampleur et de la complexité du système d'information. Ces derniers éléments influencent le nombre d'activités, la quantité de données à traiter et le nombre de gestionnaires pouvant avoir besoin de différents types d'information. Ils nous permettent de tenir également compte de l'impact économique des entreprises. 
Pour ces raisons, nous avons préféré définir la petite et moyenne entreprise en fonction:

- du nombre d'employés et de l'actif lorsqu'il s'agit d'entreprises manufacturières

- du nombre d'employés et du chiffre d'affaire lorsqu'il s'agit d'entreprises des autres secteurs d'activité et nous avons retenue la classification présentée par le Ministre délégué au PME dans son rapport intitulé "Les PME au Québec; états de la situation" (1). Cette classification vous est présentée dans les Tableau I et Tableau II (2).

\section{Tableau I}

Definition et classification suggérées des entreprises du secteur de la fabrication

Selon le nombre d'employés et le montant des actifs Québec, 1897

$\begin{array}{lll}\text { Nombre d'employes Montants des actifs } & \text { Type d'entreprises } \\ 0-49 & \text { Moins de } 3000000 \$ & \text { Petite } \\ 50-199 & 3000000 \$ \text { a } 12000000 \$ & \text { Moyenne } \\ 200-499 & 12000000 \$ \text { a } 30000000 \$ & \text { Grande } \\ 500 \text { et plus } & 30000000 \$ \text { et plus } & \text { Tres grande }\end{array}$

2. Définition de 1 a recherche-action

Étant donné que notre mandat consistait à effectuer une recherche-action, nous devons préciser ce qu'est la recherche-action. Cette précision permettra aux lecteurs de comprendre le cadre de l'étude et les facteurs qui nous ont influencés dans le choix de la méthodologie.

1- Rapport du Ministre délégué au PME, Les PME au Québec, Etat de la situation, Gouvernement du Québec, Ministère de 1'Tndustrie et du Commerce, Bibliothèque nationale du Québec, 1987, 320 pages

2- ibid. pages 223 et 224 
Tableau II

Definition et classification suggerées des entreprises des secteurs autres que manufacturiers.

Selon le nombre d'employes et le montant annuel des ventes Québec

Nombre d'employes Ventes annuelles Type d'entreprises

Moins de 50 Moins de $2000000 \$$ Petite

50-99 $2000000 \$$ a $20000000 \$$ Moyenne

Plus de $100 \quad$ Plus de $20000000 \$$ Grande

Source: Rapport du ministre délégué aux PME, Québec, 1986.

Nous définirons la recherche-action en vous expliquant son processus, les critères

qui doivent être rencontrés pour qu'une recherche soit une recherche-action et finalement, nous vous présenterons les objectifs de la recherche-action.

Monsieur Paul Prévost définit le processus de recherche-action comme étant "un ensemble d'activités effectuées conjointement par chercheur(s) et acteur(s) et par lesquelles la science se trouve enrichie par l'action et l'action par la science."(1) II mentionne également que:

pour que la recherche-action atteigne ses objectifs, le chercheur doit demeurer dans l'organisation durant tout le processus d'investigation, le développement des actions à prendre et des stratégies de changement et leur application et évaluation. (2)

La recherche-action implique l'existence simultanée d'une recherche et d'une action et la participation conjointe de chercheurs et d'acteurs. ${ }^{(3)}$

1- PREVOST, Paul Ph.D, Le Diagnostic-Intervention: une approche systémique au diagnostic organisationnel et à la recherche-action, LEER, 7 aout 1983, Chicoutimi, page 76.

2- ibid., page 77

3- PREVOST, Paul Ph.D, Modèle cohérent d'intégration de la recherche-action au milieu socio-économique, Chicoutimi, LEER, 1982, page 11 
Un autre auteur mentionne que "la recherche-action est un travail qui s'inscrit dans une certaine durée, ce travail ne se réduit pas à des interventions ponctuelles" (1).

Donc, pour effectuer une recherche-action, nous devons avoir une interaction entre les gens oeuvrant à l'intérieur de l'organisation et le chercheur ou la personne qui effectue une intervention. Ce chercheur doit demeurer dans l'organisation tout le temps que dure l'intervention. L'intervenant doit effectuer des recherches et poser, conjointement avec les membres de l'organisation, des actions visant à améliorer la situation.

Maintenant que nous avons déterminé ce qu'est une recherche-action, nous devons en préciser les objectifs. Notre revue de littérature nous a permis de constater que la recherche-action "vise à combiner les intérêts du chercheur et des personnes directement impliquées dans le domaine abordé..." (2) et que ses objectifs "sont de contribuer à la solution de problèmes auxquels sont confrontés les intéressés, tout en contribuant à l'élaboration d'un modèle théorique" (3).

3. Méthodologie employée pour effectuer la recherche-action

Pour effectuer notre recherche-action, nous devions recourir à une méthodologie qui nous permettrait d'effectuer une recherche et une action, conjointement avec les intervenants de l'organisation. Cette méthodologie devait nous permettre de contribuer à la solution de problèmes auxquels sont confrontés les membres de l'organisation (dans

1- GRELL, P. et WERY A, Problématique de la recherche action. Extrait de:

Revue internationale d'action communautaire, no.5, printemps 1981, numéro spécial sur la recherche-action, page 124

2- GRELL, P. et WERY A., Problématique de la recherche action. Extrait de:

Revue internationale d'action communautaire, no.5, printemps 1981, numéro spécial sur la recherche-action, page 124.

3- ibid. 
ce cas, les membres du Groupe Hare1, Drouin). De plus cette méthodologie doit nous permettre de déterminer les besoins d'information et ce, en tenant compte du risque associé au projet.

3.1 Evaluation de l'incertitude et sélection d'une stratégie

Pour déterminer le risque associé au projet et sélectionner la stratégie et la méthodologie appropriées, nous avons employé la technique proposée par Davis ${ }^{(1)}$. Ce dernier nous présente quatre (4) stratégies dont la sélection dépend de:

- des caractéristiques de l'organisation

- de l'incertitude reliée au processus d'analyse des besoins.

\subsubsection{Evaluation de l'incertitude globale du projet}

Pour évaluer l'incertitude reliée au processus d'analyse des besoins, Davis nous propose un procédé composé de cinq (5) étapes. Ces étapes vous sont résumées dans le Tableau III, Tableau IV.

Ce procédé nous suggère quatre (4) éléments (système objet, type application, utilisateurs, analystes) dont l'ensemble des caractéristiques génèrent trois (3) incertitudes (stabilité des besoins en information, capacité des utilisateurs à exprimer leurs besoins et capacité des analystes à obtenir les besoins et à les évaluer). Ces incertitudes inter-agissant ensemble déterminent le niveau d'incertitude globale du projet. mentionnés.

1- DAVIS, Gordon B. et a1., Système d'information pour le management, Edition G. Vermette inc., Bouchervil1e, 1986, Volume 1, 332 pages 
Tableau III

\begin{abstract}
1. Définir les caract éristiques

de la situation

Quatre êlêments

affectant

l'incertitude de

la détermination

des besoins en

information
\end{abstract}

1. Système objet

2. Type

d'application

3. Utilisateurs

4. Analystes

$\begin{array}{ll}\text { 2. Evaluer les } & \text { 3. Evaluer } \\ \text { processus } & \text { i incertitude } \\ \text { incertains } & \text { globale }\end{array}$

Trois incertitudes dans le processus de définition des besoins

$\begin{array}{ll}\begin{array}{l}\text { 4. Choix de } \\ \text { stratêgie }\end{array} & \begin{array}{l}\text { 5. Choix } \\ \text { des } \\ \text { méthodes }\end{array} \\ \begin{array}{l}\text { Incertitude } \\ \text { faible }\end{array} & \begin{array}{l}\text { aéthode } \\ \text { Enquête }\end{array} \\ & \text { méthode }\end{array}$

Systène

mêthode

Existence et

stabilité des besoins déjà

satisfaits

aéthode
Capacité des util isateurs à expriwer leur besoins

Coupétence des analystes à recueillir et êvaluer les besoins
Incertitude globale dans le processus de définition des besoins
Caractéris- aéthode tiques du système opérant wéthode

Expéri-
mentation
avec
prototype méthode

La première étape consiste à identifier les caractéristiques des quatre éléments. La stabilité du système objet (1), sa définition, la quantité de changements effectués à l'intérieur de ce dernier, la programmation des activités et la structure des décisions, la traditionnalité de l'application, sa simplicité, le niveau décisionnel qu'elle supporte, l'expérience des utilisateurs face aux systèmes d'information, la

1- (composé d'individus, de machine, de biens, d'argent, etc.) DAVIS, Gordon $B$. et a1., Système d'information pour le management, Edition G. Vermette inc., Boucherville, 1986, Volume 1, page 260 
formation et 1'expérience des analystes oeuvrant sur le projet sont toutes des caractéristiques qui génèrent trois incertitudes qui affectent le niveau global d'incertitude du processus d'analyse de besoins.

La seconde étape consiste à évaluer l'effet des trois incertitudes sur l'incertitude globale du projet. Davis ${ }^{(1)}$ mentionne:

que l'incertitude sur la stabilité des besoins en information dépend par exemple:

1. De l'absence de modèle du système objet, à cause d'objectifs confus, d'une organisation floue, de procédures mal définies.

2. Du manque de stabilité de la structure du système objet et des tâches qui y sont faites.

3. Du manque de stabilité dans l'utilisation du système d'information. Les activités non programmées, sans structure prédéfinie et les changements dans le personnel utilisateur créent des profils d'utilisation instables.

4. De l'existence d'un grand nombre d'utilisateurs. Le grand nombre peut affecter la stabilité des besoins s'il n'existe pas un mécanisme d'arbitrage entre les demandes divergentes afin de parvenir à un consensus.

Il mentionne également que la capacité des utilisateurs à exprimer leurs besoins est en fonction des limites humaines et des caractéristiques de l'application. Ainsi le manque de modélisation des activités ou des décisions, la complexité du système objet, le nombre d'utilisateurs, le type d'utilisateurs et leur expérience influencent le degré d'incertitude. Des utilisateurs expérimentés, un systẻme modélisé, un nombre restreint d'utilisateurs avec un niveau de participation élevée auront comme conséquence de réduire le niveau de l'incertitude.

Finalement, l'incertitude reliée à la capacité des analystes à obtenir les besoins d'information et à les évaluer dépend de la formation générale des analystes et de leur expérience dans un domaine similaire.

1- DAVIS, Gordon B. et a1., Système d'information pour le management, Edition G. Vermette inc., Boucherville, 1986, Volume 1, page 268 
La troisième étape consiste à évaluer l'incertitude globale en tenant compte de tous les éléments mentionnés jusqu'à présent. A la quatrième étape, nous choisissons une stratégie principale et une méthode.

3.1.2 Stratégie employée selon 1'incertitude globale du projet

Selon le résultat de l'évaluation de l'incertitude globale du projet, nous disposons de quatre stratégies: l'enquête, le système existant, les caractéristiques du système opérant et expérimentation par prototype.

L'enquête est employée lorsque le niveau d'incertitude est bas. Dans cette stratégie, l'analyste identifie les besoins d'information en interrogeant les usagers du système. Cette stratégie peut être employée seulement lorsque les usagers connaissent bien le système, lorsqu'ils sont capables de définir les informations dont ils ont besoins en des termes très détaillés.

Le système existant est une stratégie qui consiste à analyser les activités et fonctions d'un système existant, qu'il soit à l'intérieur de l'organisation, existant dans une organisation semblable, ou décrit dans des volumes de référence. Très utilisée par le passé, cette stratégie qui présentait le risque de nous empêcher de percevoir les évolutions souhaitables dans le système d'information, est maintenant employée à titre de stratégie complémentaire à la stratégie basée sur les caractéristiques du système opérant.

La stratégie basée sur les caractéristique du système opérant repose sur les caractéristiques du système objet et sur le fait que les besoins d'information découlent des activités du système-objet. "Donc la méthode la plus logique et la plus complète pour obtenir les besoins en information semble consister à analyser les caractéristi- 
ques de ce système objet." (1) Diverses méthodes nous sont proposées à l'intérieur de cette stratégie. Certaines de ces méthodes sont orientées plutôt vers l'organisation, d'autres vers les applications. Le Tableau IV ${ }^{(2)}$ vous présente neuf de ces méthodes en vous indiquant leur orientation.

Tableau IV

METHODE

PLUTOT VERS

L " ORGANISATION

PLUTOT VERS

L'APPLICATION

Analyse normative

$\mathbf{x}$

Analyse de la straté-

$\mathbf{x}$

gie de l'entreprise

Analyse des facteurs

essentiels de succès

$\mathbf{x}$

Analyse des processus $\quad x$

de travail

Analyse des fins et $x$

des moyens

Analyse des décisions $x$

Analyse socio-technique $\quad x$

Analyse des entrées - $x$

traitements - sortie

Réel représenté $\quad x \quad x$

L'expérimentation par prototype est employée lorsque que le niveau d'incertitude est élevée. Cette stratégie consiste à définir le système en même temps que les besoins

1-DAVIS, Gordon B. et al., Système d'information pour le management, Edition $G$. Vermette inc., Boucherville, 1986, Volume 1, page 261.

2- DAVIS, Gordon B. et al., Système d'information pour le management, Edition G. Vermette inc., Bouchervil1e, 1986, Volume 1, page 261. 
se développent. Elle repose sur les principes que les usagers ne sont pas en mesure de définir leurs besoins sans avoir essayé le système (effet d'apprentissage) et que l'analyste dispose d'outils lui permettant de développer et de modifier le système au fur et à mesure que les besoins se définissent.

\subsection{Analyse de l'organisation}

\subsubsection{Présentation de l'organisation}

Le groupe Harel, Drouin est composé d'une société de comptables (Harel, Drouin \& Associés) agréés offrant principalement des services de vêrification et de fiscalité, d'une corporation offrant des services de gestion (Harel, Drouin Gestion Conseils), d'une corporation offrant des services informatiques (La Société d'informatique de gestion). A toutes ces entités s'ajoute une corporation de gestion (Les Services Harel, Drouin inc.) qui a été créé pour des bénifices fiscaux.

La Société Harel, Drouin \& Associés existe depuis une douzaine d'années et elle est issue de la fusion de deux sociétés de comptables agréés. Les deux associés fondateurs des deux sociétés fusionnées sont encore actifs au sein de la Société Harel, Drouin. Les décisions majeures concernant la Société sont prises par l'Assemblé des associés. Cette assemblé a lieu mensuellement. Les décisions d'opérations sont prises par le comité de direction qui est composé de trois associés ayant plusieurs années d'expérience. Les décisions concernant les employés ou les dossiers des clients sont prises par l'associé qui a la responsabilité du client et de l'employé.

La Société d'informatique et de gestion fut créé au début des années 1980 par des associés de Harel, Drouin \& Associés. Harel, Drouin Gestion Conseils existe depuis environ trois ans et fut créé suite à l'augmentation du nombre de mandats de gestion. 
La Société Harel Drouin est composée d'un service de vérification, d'un service de fiscalité, d'un service de contrôle de la qualité et d'un service d'administration. Le service d'administration s'occupe des achats concernant toutes les entités du groupe Harel, Drouin et traitent toutes les données comptables. Ce service est également responsable de la production des états financiers de chaque entité.

Le directeur du service de l'administration travaille pour la Société Harel, Drouin depuis dix ans. Durant les cinq premières années, il travailla dans le service de vérification. Son expérience se résume à tenir les livres comptables des clients et à vérifier des états financiers. Durant les cinq autres années, il occupa le poste de directeur administratif. Ses principales activités consistent à exercer un contrôle interne, à superviser le traitement des données comptables, à s'assurer que les rapports comptables soient produits selon les échéanciers et à produire les états financiers. Il supervisent le travail de trois employés. Ces trois personnes travaillent au sein du service de l'administration depuis respectivement sept, quatre et trois ans. Elles ont donc toutes une bonne connaissance des tâches et fonctions reliêes à leur travail.

3.2.2 Présentation du système d'information comptable (système objet) des entreprises du groupe Hare1, Drouin

Le système d'information comptable du groupe Harel, Drouin est composé de transactions concernant toutes les sociétés ou corporations mentionnées précédemment auxquelles nous devons ajouter quelques transactions concernant d'autres corporations dont la charte existe mais qui exercent très peu d'opérations.

Comme dans tous les systèmes comptables, nous retrouvons des grand-livres, des auxiliaires des comptes à payer, des auxiliaires des comptes à recevoir, des caisses recettes et des caisses déboursés. A tous ces registres comptables nous devons ajouter des auxiliaires des travaux en cours. Ces auxiliaires fonctionnent selon le même 
principe que les autres auxiliaires. Ils sont utilisés pour enregistrer les revenus qui sont produits à partir du temps que les employés et associés travaillent dans les dossiers de leurs clients. On retrouve des auxiliaires de travaux en cours dans les firmes d'avocats, de construction, d'ingénierie. Même si ces auxiliaires ne sont pas aussi souvent utilisés que les auxiliaires des comptes à recevoir et à payer, leur utilisation en est très simple. Pour enregistrer le temps travaillé dans le dossier d'un client, nous débitons le compte travaux en cours du client et nous créditons le compte revenus. Lorsque nous facturons le client, nous créditons le compte de travaux en cours du client et débitons son compte à recevoir. Le report des transactions dans le grandlivre s'effectue de la même façon qu'avec les autres auxiliaires. La comptabilité des entreprises du groupe Harel, Drouin est donc une application très traditionnelle. Elle est simple et elle aide au travail administratif.

3.3 Évaluation de l'incertitude globale du processus d'analyse des besoins d'information des entreprises du Groupe Harel, Drouin

Pour évaluer l'incertitude globale du processus d'analyse des besoins d'information comptable nous allons, dans un premier temps, vous présenter les caractéristiques de l'organisation. Ensuite, nous allons identifier les caractéristiques du système objet, de l'application, des utilisateurs et des analystes (voir Tableau III, Tableau IV) et évaluer le niveau des trois incertitudes et leur influence sur l'incertitude globale du processus d'analyse des besoins d'information comptable des entreprises du Groupe Harel, Drouin. Les résultats de notre analyse vous sont présentés dans le Tableau V. 


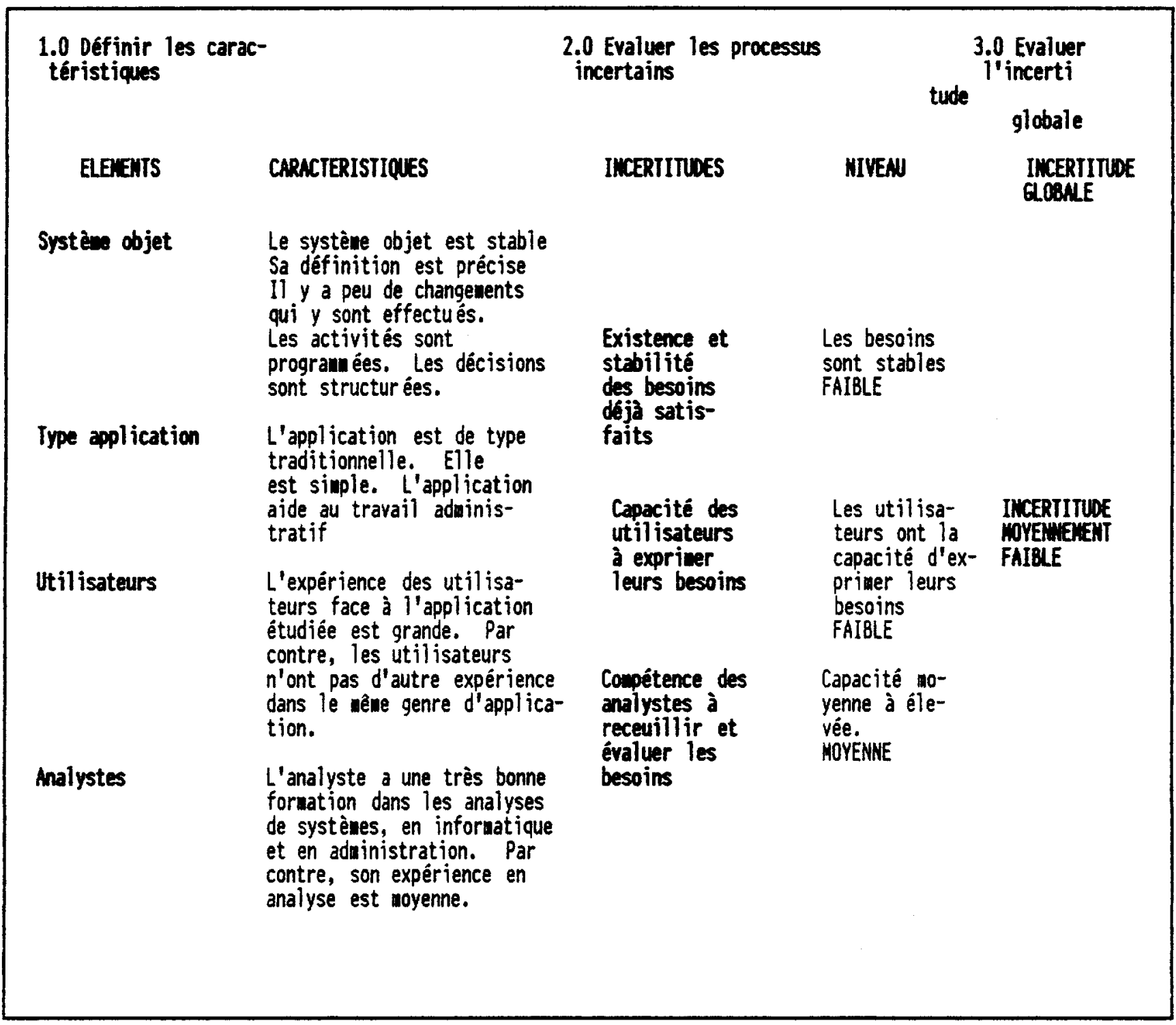

\subsection{Stratégie et méthodologie employées}

Les caractéristiques des éléments du système objet nous a permis d'évaluer le niveau d'incertitude du projet. L'incertitude est moyennement faible (voir Tableau V). Dans le cas d'un niveau d'incertitude moyennement faible nous devrions employer la stratégie basée sur le système existant. Cette stratégie a longtemps été utilisée par le passé mais maintenant nous l'utilisons comme "stratégie complémentaire avant une autre méthode portant sur le système opérant. En effet, on risque, par l'analyse du système 
existant, de ne pas percevoir les évolutions souhaitables dans le système d'information."

Nous allons donc employer la stratégie basée sur les caractéristiques du système opérant. Nous utiliserons également, comme stratégie complémentaire, la stratégie du "système existant". Cette dernière nous permettra:

- de bien connaître le fonctionnement du système utilisé actuellement dans l'organisation

- d'identifier ses forces et faiblesses.

La stratégie basée sur les caractéristiques du système opérant offre la possibilitê d'utiliser des méthodes basées sur l'organisation ou sur l'application (voir Tableau IV). Étant donné que notre recherche-action concerne le système d'information comptable du groupe Harel, Drouin (une application), nous avons eu recours à la méthode qui permet d'analyser les entrées, les traitements et les sorties. Elle consiste à effectuer:

une analyse systémique des flux et des transformations de données. On commence par une vue globale du système-objet. On décompose ce système en sous-système qui sont eux-mêmes éclatés. Cette décomposition se poursuit jusqu'a ce que les activités du traitement de l'information soient définies comme des activités spécifiques à l'intérieur d'un soussystème. (2)

1- DAVIS, Gordon B. et al., Système d'information pour le management, Edition G. Vermette inc., Boucherville, 1986, Volume 1, page 260.

2- DAVIS, Gordon B. et a1., Système d'information pour le management, Edition G. Vermette inc., Boucherville, 1986, Volume 1, page 265 
3.5 Description de la méthodologie des diagrammes de flux d'information

Pour décomposer chacun des sous-systèmes jusqu'à l'obtention d'activités spécifiques, nous avons eu recours aux graphiques proposés par Gane et Sarson ${ }^{(1)}$. Vous trouverez dans les annexes les graphiques de chaque sous-système composant le système d'information comptable. A l'intérieur de chaque graphique sont illustrées les activités spécifiques de chaque sous-système. Vous trouverez également les flux d'information reliant les activités entre elles. La description des activités et des flux de données est présentée à la suite du graphique.

Ces graphiques sont réalisés à l'aide de quatre figures (voir Tableau VI). Les graphiques sont employés pour illustrer tous les sous-systèmes composant le système d'information et la circulation des données entre chaque sous-système.

Gane et Sarson proposent de représenter l'éclatement (1a subdivision) d'un soussystème en dessinant:

les diagrammes de flux de données à l'intérieur des frontières que symbolise le rectangle du traitement du plus haut niveau. Bien évidemment toutes les flèches du flux de données qui entrent ou qui sortent du rectangle représentant le traitement au niveau supérieur doivent franchir les côtés du rectangle. (2)

Les stockages de données sont représentés à l'intérieur des limites du rectangle seulement s'ils sont créés ou consultés pour ce traitement et pour aucun autre. ${ }^{(3)}$

Les diagrammes de Gane et Sarson doivent être complétés par un dictionnaire dans lequel nous devons retrouver:

- la description détaillée du flux et les données ou structures de données le composant

1- GANE Chris, SARSON Trish, Analyse structurée des systèmes: outils \& techniques, ImpSysTech $S . A ., 1980,445$ pages

2- GANE Chris, SARSON Trish, Analyse structurée des systèmes: outils \& techniques, ImpSysTech S.A., 1980, page 62.

3- Ibid, page 63. 


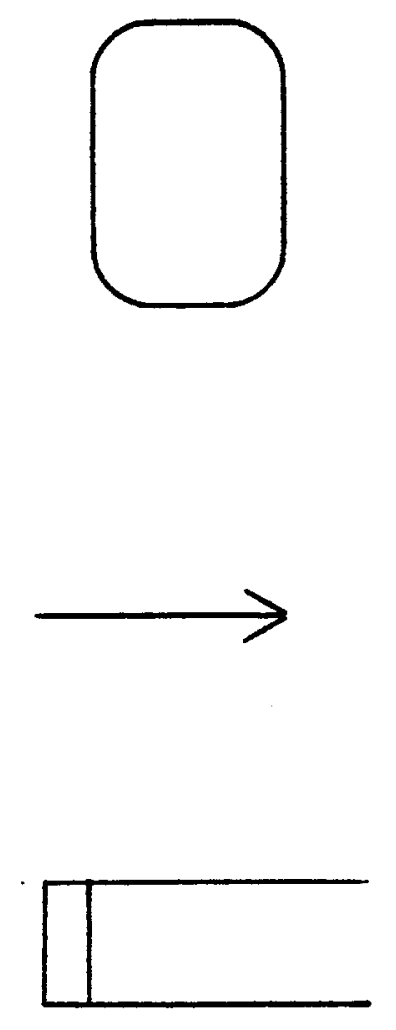

Cette figure représente une activité du système d'information. L'activité est decrite en détail dans le dictionnaire. A l'interieur d'une activite, des données sont transformees pour produire:

- d'autres données qui seront transformées à nouveau par une autre activité

- de l'information qui sera destiné a l'utilisateur.

Les activites sont identifieses par leur numero et l'inscription inscrites à l'intérieur de la figure.

Cette figure représente un flux de données ou d'information. Les flux proviennent d'une entité externe, d'une activité ou d'un stockage de donnees et vont vers un utilisateur externe, vers une autre activité ou vers un stockage. Ils sont identifies par leur origine et destination et portent un nom qui est inscrit près de la figure.

Cette figure représente un stockage de données. Les stockages de donnees sont identifiés par la lettre "D", le numéro de l'activité auquel ils sont reliés, un "/" et un numéro quelconque.

Cette figure représente une entité externe au système d'information. Les entites sont généralement des origines ou des destination de flux de données. Elles représentent généralement des utilisateurs internes ou externes.

- la description de chaque activité et un résumé de sa logique

- la description de chaque stockage et les données ou structures de données le composant

- la description de chaque entité externe. 
3.6 Description de la méthodologie utilisée pour réaliser l'étude

Pour réaliser l'analyse des besoins d'information comptable, nous avons décomposé le système d'information comptable en plusieurs sous-systèmes.

Sachant que les systèmes d'information comptable produisent généralement les registres suivant:

- auxiliaire des comptes à payer

- auxiliaire des comptes à recevoir

- auxiliaire des travaux en cours(1)

- journal des ventes

- journal des achats

- journal général

- caisse recettes

- caisse déboursés

et les rapports financiers suivants:

- bilan

- état des résultats,

nous avons identifié et analysé les sous-systèmes générant les registres et rapports mentionnés.

1- L'auxiliaire des travaux en cours est produit seulement dans les entreprises de services dont le revenu est basé sur le temps travaillé. 
* compiler les feuilles de temps: permet l'enregistrement des revenus

* enregistrer les ventes: génèrent les transactions permettant de produire le journal des ventes

* enregistrer les recettes: génèrent les transactions permettant de produire le caisse recettes

* préparer la paie: génèrent les transactions permettant de produire l'auxiliaire des comptes à payer et le caisse déboursés

* payer les factures des fournisseurs: génèrent les transactions permettant de produire l'auxliaire des comptes à payer et le caisse déboursés.

* produire les etats fianciers: consiste à compiler les transactions pour produire l'éats des résultats et le bilan.

Notre analyse a été réalisée en deux étapes: la première consistait a analyser les données et fonctions du système existant; la seconde à analyser les caractéristiques du système opérant ${ }^{(1)}$.

Pour analyser les données et fonctions du système existant, nous avons dressé la liste des sous-systèmes (voir Tableau VII) dont les objectifs étaient de réaliser les registres et rapports mentionnés précédemment. Nous avons employé les graphiques de

1- système qui repose sur les caractéristiques du système objet. 
Gane et Sarson afin de visualiser les différentes fonctions exécutées et les données qui sont traitées. Cependant, nous n'avons pas, comme le suggère la méthode, identifié . les flux d'information reliant les sous-systèmes les uns aux autres. Il était impossible d'identifier les flux d'information qui relient les sous-systèmes entre-eux. Nous avons donc analyser chacun des sous-systèmes indépendamment et pour chaque sous-système, nous avons:

- demandé aux usagers de nous décrire les tâches exécutées

- demandé aux usagers de nous fournir des exemplaires des documents utilisés

- dressé un ou plusieurs (dans le cas d'éclatement) diagrammes de flux de données

- rédigé le dictionnaire ${ }^{(1)}$ dans lequel nous retrouvons la description détaillée des flux et des activités (2).

L'analyse des caractéristiques du système opérant a été réalisée à l'aide de la méthode de l'analyse des entrées-traitements-sorties (voir Tableau IV qui présente plusieurs méthodes qui peuvent être utilisées à l'intérieur de la stratégie basée sur l'analyse des caractéristiques du système opérant). Cette méthode est "une analyse systémique des flux et des transformations de données....'analyse structurée avec les diagrammes de flux de données ... est un bon exemple d'une telle méthode" ${ }^{\prime(3)}$.

Nous avons donc utilisé l'analyse structurée pour analyser les caractéristiques du système opérant. L'analyse structurée a été réalisée également à l'aide des diagrammes de flux d'information de Gane et Sarson. Nous avons choisi d'utiliser ces diagrammes parce qu'ils offrenent la possibilité d'illustrer le système d'information dans son ensemble. Ils nous offrent des figures qui nous permettent d'identifier clairement les entités externes à notre système d'information, les regroupements de données, les flux

1-Vous trouverez les graphiques et le dictionnaire dans les annexes.

2- La description détaillée des stackages de données du système existant n'a pas encore été réalisée; elle sera ultérieure au dépôt de cet ouvrage.

3- DAVIS, Gordon B. et al., Système d'information pour le management, Edition G. Vermette inc., Boucherville, 1986, Volume 1, page 266. 
de données et les processus de traitement. Il existe d'autres méthodes d'analyse structurée. Une de ces méthodes illustre le système d'information à l'aide de cercle et de flèches. Il est très difficile de représenter les processus de traitement et de transformation des données dans des diagrammes qui ne contiennent que des cercles et des flèches. Gane et Sarson mentionne ceci:

In analysis..., we need to recognize external entities and data stores as well as data flows and transforms or processes. To represent our logical system fully, we need to add symbols to the simple program graph. Also, since we need to describe our transforms or process clearly and it is hard to get much legible writing inside a cirgle, we adopt a rectangle with rounded corners as the process symbol.

Les diagrammes de Gane et Sarson ont donc été utilisés pour analyser le système existant dans 1'organisation et pour analyser les caractéristiques du système opérant. Ils ont été modifiés jusqu'a ce qu'ils représentent:

- dans le cas du système existant, le système d'information opérant dans 1 'organisation

- dans le cas des caractéristiques du système opérant, un système d'information répondant aux besoins d'information de 1 'organisation.

\subsection{Outils disponibles}

Pour effectuer 1 'analyse des besoins d'information des entreprises du Groupe Hare1, Drouin, et développer un système d'information répondant aux besoins, nous avons entrepris des recherches afin de découvrir un logiciel qui nous permettrait de:

- développer et modifier facilement les graphiques correspondant à ceux suggérés dans la méthode de Gane et Sarson.

- déplacer facilement les figures des graphiques en redisposant les flux

1- GANE Chris, Sarson Trish, Structured Systems Analysis: tools and techniques, Improved System Technologies, Inc., New York, N.Y., 1979, page 25. 
reliant les figures les unes aux autres.

- générer les éléments composant le dictionnaire afin qu'il n'y ait aucun oub1i.

Le logiciel recherché devait fonctionner sur l'équipement qui est à la disposition de 1'analyste. Etant donné que le logiciel ne serait probablement pas utilisé après 1 'achèvement de 1 'étude et qu'il était possible que l'analyste ait à supporter le cout du logiciel, le prix de ce dernier devait s'harmoniser avec le budget disponible.

Nos recherches effectuées auprès de firmes oeuvrant dans le développement de système d'information, dans le développement de logiciels, auprès d'universités reconnues nous ont permis de connâtre l'existence des logiciels suivants:

- Excelerator

- Consoi - MCD 1 1)

- Easyflow (2).

Plusieurs mois après le début de l'analyse, nous avons découvert le logiciel Silverrun - DFD'3) qui fonctionne sur les ordinateurs Macintosh.

\footnotetext{
1- MCD = Modèle de conception de données

2- Produit par HavenTree Software Limited P.O. Box 2260 Kingston, Ontario $K 7 L 5 J 9$ Canada

3- DFD = Diagrammes de flux de données Produit par: Peat Marwick Technologies

1175 , avenue Lavigerie suite 090 Sainte-Foy, (Québec) GIV $4 P 1$ té1.: 418-657-5582
} 


\subsubsection{Excelerator}

Nous n'avons pas réussi à obtenir beaucoup d'information au sujet de ce logicie1. Nous ne savons pas selon quelle méthode il permet de développer des systèmes d'information et nous n'avons pas pu le voir en opération. La documentation fournie par 1'Université du Québec à Montréal était incomplète. Les seules informations obtenues sont les suivantes:

- le logiciel fonctionne sur ordinateur compatible IBM

- son cout est d'environ $10000 \$$

- son utilisation a nécessité à un usager 4 mois d'apprentissage

\subsubsection{Logicie1 Consoi-MCD}

Le logiciel Consoi-MCD est un outil de conception assistée par ordinateur basé sur la conception de données utilisant le formaliste individuel ou entitérelation de la méthode Merise. I1 est donc d'aucune utilité pour effectuer des études concernant I'analyse des entrées-traitements-sorties. 
3.7.3 Logicie1 Easyflow

Ce logiciel est développé par HavenTree Software' 1 '. I1 permet de réaliser des ordinogrammes en sélectionnant des figures. Parmi les figures proposées par le logiciel, nous retrouvons trois des quatre figures (voir Tableau VI) utilisées dans le cadre de la méthode de Gane et Sarson. Le logiciel permet également :

- de tracer des lignes reliant les figures les unes aux autres

- d'inscrire des chaînes de caractères à 1'intérieur ou à 1 'extérieur des figures

- de déplacer facilement les figures ou groupe de figures.

Cependant, ce logiciel ne réorganise pas les flux de données (lignes reliant les figures les unes aux autres) lorsque des figures sont déplacées et n'offre aucune aide pour la production du dictionnaire. Son principal avantage réside dans son cout (150\$) et dans le fait qu'il permet de présenter aux usagers des diagrammes sans bavure. Pour produire le dictionnaire, 1'analyste doit dresser lui même la liste des processus, des flux de données, des stockages et des entités externes.

Des oublis peuvent donc se glisser. L'ajout, l'élimination, la modification de flux de données (changement de nom ou de destination), de processus, de stockages entrainent des modifications laborieuses du dictionnaire.

1- HavenTree Software Limited P.O. Box 2260

Kingston, Ontario K7L $5 \mathrm{Jg}$

Canada 
3.7.4 Logicie1 Silverrun - DFD

Le logiciel Silverrun - DFD (découvert plusieurs mois après le début de l'analyse) est un logiciel fonctionnant sous la technologie Macintosh. C'est un outil de conception assisté par ordinateur pour la construction de diagrammes de flux de données selon la méthode développée par Gane et Sarson. Ce logiciel a été conçu par Peat Marwick Technologies. La documentation accompagnant le logiciel mentionne qu'il:

\begin{abstract}
apporte des gains de productivité de l'ordre de 50\% grâce à l'intégration et des diagrammes, et à l'aide qu'il apporte pour garantir la cohérence des diagrammes. La simplicité et la rapidité des modifications des spécifications que permet silverrun - DFD donne une nouvelle dimension à "I'analyse structurée" dont l'utilisation a été limitée à cause de la lourdeur de la documentation qu'elle entraine, en particulier dans la mise à jour des diagrammes et de la documentation qui les accompagnent.
\end{abstract}

Nous avons essayé ce logiciel. Sans pouvoir affirmer que le lógiciel permet effectivement des gains de productivité de l'ordre de $50 \%$, nous avons constaté qu'il s'opère facilement et que son exploitation maximale peut être obtenue, par une personne familière avec les concept de la micro-informatique, dans un délais variant entre 24 et 48 heures d'opération.

Ce logiciel permet de créer les diagrammes de flux de données, d'éclater un processus et de générer le dictionnaire dans une seule opération. Les flux de données sont réorganisés dès qu'une figure est déplacée. Le dictionnaire est modifié dès l'instant où des changements (ajout des processus, de flux de données, d'entités externes ou de stockages etc.) sont apportés au diagramme. La numérotation de toutes les figures est automatique. Nous croyons donc que cet outil est efficace, qu'il améliore la qualité du travail de l'analyste et augmente sa productivité. 
Nous avons tenté d'entrer en communication avec la compagnie Peat Marwich technologies afin de connaitre le prix de vente du logiciel et pour savoir s'il existait une version fonctionnant sur les ordinateurs compatibles IBM. Malheureusement, au moment d'écrire ces lignes, nous avons été dans 1'impossibilité d'entrer en communication avec la compagnie. Nous avons demandé à la firme de comptables agrées Peat Marwick si cette compagnie leur était apparentée. La firme nous a mentionné qu'elle en ignorait l'existence. L'assistance annuaire n'a aucun abonné correspondant à cette compagnie.

\subsection{Outil utilisé}

Comme vous 1'avez sans doute deviné, pour réaliser 1'analyse des besoins d'information des entreprises du Groupe Hare1, Drouin, nous avons eu recours au logiciel Easyflow'1). A 1'époque où l'analyse fut amorcée, Easyflow était le seul logiciel fonctionnant sur les ordinateurs compatibles IBM, qui était financièrement accessible et qui permettait de concevoir et de modifier des graphiques plus rapidement qu'avec un dactylo.

Etant donné que nous retrouvons, parmi les figures contenues dans le logiciel, les figures utilisées pour développer des diagrammes de flux d'information selon la méthode de Gane et Sarson, nous croyons que le logiciel aurait avantage à être amélioré de façon à:

- permettre de réorganiser le diagramme de flux de données lorsqu'un processus, stockage ou entité externe est déplacé

- produire le dictionnaire de données en:

1- Produit par HavenTree Software Limited P.O. Box 2260

Kingston, Ontario KTL $5 J 9$

Canada 
- élaborant la liste des flux de données dans laquelle on retrouverait le nom de chaque flux de données, son origine et sa destination

- en définissant le contenu des stockages de données à partir du contenu des flux de données qui y sont dirigés et ce, en indiquant à 1 'analyste les incohérences.

4. Connaissances à tirer du point de vue méthodologique, théorique et conceptuel de 1'expérience vécue

4.1 Utilisation de la méthodologie chez les entreprises du groupe Hare1 Drouin

Avant de traiter de l'applicabilité de la méthodologie chez Hare1, Drouin, nous allons déterminer le type de l'entreprise. Nous allons donc nous référer à la classification proposée par le Ministre délégué au PME(1). Selon cette classification, nous considérons la Société Hare1, Drouin' 2 ) comme étant une moyenne entreprise, la corporation Hare1, Drouin Gestion Conseil comme étant une petite entreprise et la Société d'informatique de gestion comme étant une petite entreprise.

1- voir Tableau II

2- La Société Hare1, Drouin détient une corporation de gestion dont 1 'existence n'est justifiée que pour les avantages fiscaux qu'elle rapporte. Pour cette raison, nous avons inclu les opérations de cette corporation à celle de la société. 


\subsubsection{Etude du système existant}

L'analyse systémique demande que l'analyste commence par une vue globale du système objet. Les systèmes composant 1a vue globale sont décomposés en soussystèmes qui sont éclatés jusqu'à ce que les activités du traitement de l'information soient des activités spécifiques à l'intêrieur d'un sous-système.

Pour réaliser l'étude du système existant, à partir des documents et rapports produits et utilisés, nous avons identifié chaque sous-système composant le système d'information comptable. L'analyse a débuté par une vue globale de chaque sous-système composant le système d'information comptable. Pour chaque sous-système, nous avons dessiné les diagrammes de flux d'information et éclatés chaque diagramme jusqu'à l'obtention d'activités spécifiques. Les diagrammes ont été révisés conjointement par le chercheur et les intervenants. I1s ont été modifiés jusqu'à ce qu'ils représentent le système existant tel que perçu par les personnes mentionnées précédemment. Par la suite, nous avons rédigé le dictionnaire.

Au début de l'analyse, nous n'avions aucun élément qui nous permettais d'avoir une vue globale du système. L'outil utilisé pour réaliser l'étude ne nous permettais pas de dessiner, à partir des diagrammes de flux d'information des sous-systèmes, le diagramme de flux d'information qui représentait le système globale.

L'êtude d'un système d'information comptable ne nous permet pas, comme I'exige la méthode de Gane et Sarson (voir page 18), de placer sur la frontière du processus étudié, tous les stockages de données utilisés par le processus qui est éclaté et par les autres processus. Dans un système d'information compta- 
ble, la très grande majorité des stockages contiennent de l'information qui est employée par l'ensemble des activités du système. Il est donc physiquement impossible de placer tous ces stokages sur la frontière du processus éclaté.

\subsubsection{Analyse des caractéristiques du système opérant}

L'analyse des caractéristiques du système opérant a été réalisée avec la même méthode que celle utilisée pour l'étude du système existant. Le diagramme du niveau supérieur qui contient tous les sous-systèmes du système d'information comptable répondant aux besoins d'information sera réalisé ultérieurement à 1 a rédaction de cet ouvrage. Vous trouverez les diagrammes et dictionnaires de chaque sous-système composant le système d'information comptable en annexe.

\subsubsection{Amélioration de la méthodologie}

L'étude du système existant et l'analyse des caractéristiques du système opérant pourraient être réalisés beaucoup plus rapidement avec un logiciel qui permettrait de dessiner les diagrammes de flux d'information en partant du niveau supérieur au niveau inférieur. Ce logiciel devrait modifier automatiquement les diagrammes des niveaux supérieurs lorsque nous changeons les diagrammes des niveaux inférieurs. I1 devrait également générer et de modifier les dictionnaires des niveaux supérieurs à partir des dictionnaires des niveaux inférieurs.

La méthode devrait être modifiée de façon à pouvoir identifier les stockages de données partagés par d'autres processus sans les mettre sur la frontière du processus éclaté. Les stockages partagés par d'autres processus pourrait être identifiés par une numérotation distincte et/ou par l'utilisation de couleur. 
4.2 Autres éléments à ajouter à la méthodologie

Nous croyons, à partir de l'expérience vécue, qu'il est nécessaire d'ajouter la notion de propriétaires de système et d'acteurs à la méthodologie des diagrammes de flux d'information.

Ces notions vous permettront de comprendre et d'anticiper les difficultés que peuvent rencontrer les analystes lors qu'ils effectuent l'analyse d'un système d'information. Cela vous permettra également de comprendre pourquoi il peut être difficile de vendre des services d'analyse.

\subsubsection{Notion de propriétaires de système}

Nous considérons les propriétaires de système comme étant les individus qui n'utilisent pas le système d'information mais 1 'information produite par celuici, qui ont un contrôle, qui peuvent prendre des décisions, et qui détiennent les ressources financières nécessaires à son amélioration. Si le besoin d'analyser le système d'information origine des propriétaires du système, il y a de forte chance pour que des changements soient apportés rapidement. Dans le cas contraire, beaucoup de discussions, de nombreux retards dans les délais nécessaires à la production de l'information utilisée par les propriétaires du système finiront par justifier des améliorations au système d'information.

Chez Harel, Drouin, les propriétaires du système sont représentés par les associés. Ces derniers n'effectuent aucune tâche nécessaire à la production d'information. Ils contrôlent le système en établissant les priorités relatives à la production de l'information et déterminent les délais à l'intêrieur desquels doit être produite l'information demandée. Ils détiennent les ressour- 
ces financières nécessaires à l'amélioration du système d'information mais ne sentent pas la nécessité de l'améliorer car ils ne sont pas conscients de la gymnastique exécutée pour produire l'information demandée dans les délais exigés. Ils ne connaissent pas les lacunes de leur système. Ils ne sont pas et ne veulent pas être sensibilisés aux nombreuses opérations qui doivent être exécutées pour produire l'information exigée. Le besoin d'apporter des changements au système d'information est plus intense chez les utilisateurs (acteurs) que chez les associés.

4.2.2 Notion d'acteurs (ou utilisateurs) du système

Nous considérons les acteurs comme étant les individus qui utilisent le système d'information. Ils n'ont presqu'aucun pouvoir sur les décisions relatives au système d'information. Ils effectuent les activités composant le système d'information. Ils produisent de l'information (1) à partir des données générées par différents éléments composant le système (activités, personnes de l'organisation ou externe à l'organisation). Ils sont souvent conscients des lacunes du système d'information. Sans avoir la solution aux différents problèmes et lacunes du système, ils s'interrogent sur la façon d'augmenter l'efficacité de la production de l'information.

Chez Harel, Drouin, les acteurs sont représentés par les gens travaillant au service de 1'administration. Ces derniers effectuent toutes les tâches nécessaires à la production d'information. Ils essaient de rencontrer les échéanciers exigés par les associés. Ils ne détiennent pas de ressources financières nécessaires à 1'amélioration du système d'information. Ils sont très conscients

1- Dans le cas d'Hare1, Drouin I'information consiste essentiellement en des rapports financiers et quelques rapports de gestion. 
de la nécessité de l'améliorer. Le désir d'analyser le système d'information pour $y$ apporter des modifications provient d'eux.

\subsubsection{Applicabilité de la méthode dans la petite entreprise}

La méthodologie des diagrammes des flux d'information telle qu'elle a été appliquée chez les entreprises du groupe Hare1, Drouin ne peut être appliquée dans la petite entreprise à cause des couts qu'elle entraine. De plus, dans une petite entreprise, il est possible que le risque associé à l'analyse et au développement d'un système d'information comptable soit trop êlevé pour l'utilisation de la stratégie basée sur l'analyse des caractéristiques du système opérant et de la méthode de I'analyse des entrées-traitement-sorties. A cause du risque élevé, l'utilisation de la stratégie de l'expérimentation par prototypage serait mieux adaptée. La stratégie de 1 'analyse des caractéristiques du système opérant et la méthode de l'analyse des entrées-traitements-sorties peuvent être employées dans la petite entreprise si les trois conditions suivantes sont rencontrées:

- le système objet est relativement stable (1)

- les usagers ont une certaine facilité à décrire le contenu de leurs taches

- l'analyste dispose d'un outil lui permettant d'accélérer le processus d'analyse en développant les diagrammes et le dictionnaire dans une seule opération.

Dans la petite entreprise, le système objet est souvent instable à cause de la jeunesse de l'entreprise, les usagers souvent représentés par les propriétai-

1- système où il y a des documents et pièces servant de point de départ à l'analyse. Ces documents et pièces permetteront à l'analyste d'identifiés les principales activités composant le système d'information. 
res ont de la difficulté à structurer leurs activités à cause d'un manque de formation et d'expérience et finalement 1'entreprise ne dispose pas des moyens financiers pour réaliser l'étude et mettre en place les éléments nécessaires à 1 'amélioration du système d'information.

\subsubsection{Applicabilité de la méthode dans la moyenne entreprise}

Dans 1a moyenne entreprise, la méthodologie peut être appliquée car en général, les activités sont structurées' 1 ', les utilisateurs ont une certaine facilité à exprimer leurs besoins (les utilisateurs sont plus conscients des lacunes du système en place) et l'entreprise dispose des moyens financiers nécessaires à l'étude et à l'implantation des moyens améliorant le système d'information.

Par contre, les propriétaires du système d'information (ceux qui ont un pouvoir de décision sur le système et qui disposent des moyens financiers) sont moins conscients des lacunes du systèmes car ils sont les utilisateurs de l'information produite par celui-ci. Ils seront conscients des lacunes du système lorsqu'ils réaliseront qu'une information ne peut être produite efficacement par le système en place.

1- si 1'entreprise ne s'était pas structurée au cours de son développement elle aurait probablement fermé ses portes. 
Conclusion

Nous pouvons conclure que la présente étude constitue une recherche-action pour les raisons suivantes:

- 1 'étude est constituée d'un ensemble d'activités réalisées conjointement par le chercheur (Suzy Faucher) et par les acteurs

- la science se trouve enrichie par 1'action et l'action par la science.

- l'action a été enrichie par les connaissances méthodologiques du chercheur.

- la science a été enrichie (1) par l'expérimentation de la méthodologie dans un milieu organisationnel, soit une entreprise de services

- le chercheur est demeuré dans l'organisation durant tout le temps qu'a duréé l'intervention, durant le temps du développement des processus. Au moment d'écrire ces 1ignes, I'application des processus débutait.

- l'étude visait à combler les intérêts du chercheur et des personnes directement impliquées

- les objectifs de l'étude étaient de contribuer à la solution de problèmes auxquels était confrontée l'organisation tout en contribuant à l'élaboration d'un modèle théorique.

La stratégie de 1'analyse des caractéristiques du système opérant et la stratégie qui consiste à analyser le système existant sont des stratégies qui peuvent être généralement employées dans une moyenne organisation pour effectuer 1 'analyse des besoins d'information. Ces stratégies pourront être employées si

1- Voir section 6.0 "Connaissances a tirer du point de vue méthodologique, théorique et conceptuel de l'expérience vécue." 
le système objet est stable, si sa définition est précise et si les décisions sont structurées.

L'analyse du système existant dans l'organisation permet à l'analyste de connâtre le système d'information utilisé dans 1'organisation. Cette connaissance lui permettra d'identifier les forces et faiblesses du système. En connaissant ses faiblesses, 1'analyste pourra développer un système d'information plus performant. L'analyse du système existant dans l'organisation demeure une stratégie complémentaire à $1 a$ stratégie basée sur l'analyse des caractéristiques du système opérant dans la mesure où les activités du système d'information en place sont relativement stables, structurées et documentées. Dans le cas où toutes ces conditions ne sont pas rencontrées, l'analyste perdra du temps à faire cette analyse. Il serait mieux d'analyser un système existant dans des volumes de référence ou dans une autre organisation du même genre.

Dans un contexte de petite entreprise où le système objet est instable, sa définition floue, où les décisions ne sont pas structurées, l'analyste aurait intérêt à utiliser une autre stratégie. Nous recommandons la statégie de de 1 'expérimentation par prototype. L'expérimentation doit être réalisée par étape ou par secteur d'activité. Cette expérimentation crée chez les usagers un effet d'apprentissage et l'expérimentation effectuée à l'échelle de toute l'organisation provoquera beaucoup de demandes de changements et d'ajustements. Ces demandes répresentent une trop grande charge pour un seul analyste et malheuresement, la petite entreprise québecoise n'a pas les moyens financiers pour s'offrir les services de plusieurs analystes. 


\section{Bibliographie}

BURCH, John G. Jr. et al., Information Systems: Theory and Pratice, U.S.A., Jonh Wiley \& Sons, Inc., Second Edition, 1979, 571 pages.

DAVis, Gordon B. et a1., Système d'information pour 1e management, Edition G. Vermette inc., Boucherville, 1986, Volume 1, 332 pages.

GANE Chris, SARSON Trish, Analyse structurée des systèmes: outils \& techniques, ImpSysTech S.A., 1980, 445 pages

GANE Chris, Sarson Trish, Structured Systems Analysis: tools and techniques, Improved System Technologies, Inc., New York, N.Y., 1979, 241 pages.

PREVOST, Paul Ph.D, Le Diagnostic-Intervention: une approche systémique au diagnostic organisationnel et à la recherche-action, LEER, 7 aout 1983, Chicoutimi, 92 pages.

PREVOST, Paul Ph.D., Modèle cohérent d'intégration de la recherche-action au milieu socio-économique, Chicoutimi, LEER, 1982, 94 pages.

ST-PIERRE, Armand, Les systèmes d'information comptables et de gestion, Une orientation informatique, Canada, Lidec inc., 1985, 877 pages.. 


$$
\begin{aligned}
& \text { Université du Québec } \\
& \text { en Abitibi-Témiscamingue }
\end{aligned}
$$

\author{
Volume II \\ DESCRIPTION, ANALYSE ET DEVELOPPEMENT \\ D'UN SYSTEME D'INFORMATION COMPTABLE \\ POUR LES ENTREPRISES DU GROUPE \\ HAREL, DROUIN
}

Par

Suzy Faucher

B.A.A. Adm.A.

Novembre 1990 
TABLE DES MATIERES

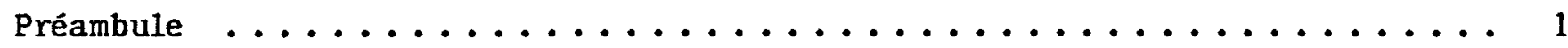

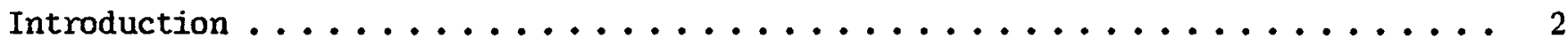

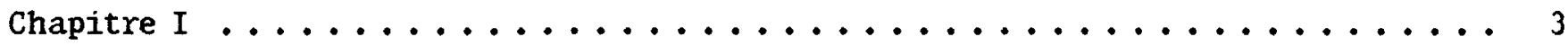

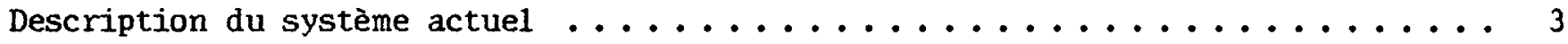

1. Présentation générale du système actuel ...................... 3

2. Compiler feuilles de temps ............................ 5

2.1 Enregistrer les données de base ....................... 5

2.2 Enregistrer données pour feuille de temps ................. 5

2.3 Imprimer 1 la feuille de temps $\ldots \ldots \ldots \ldots \ldots \ldots \ldots$

2.4 Vérifier la feuille de temps .......................... 8

2.10 Approuver la feuille de temps ....................... 8

2.5 Procéder à la fermeture de la semaine ...................... 9

2.6 Produire le sommaire mensuel ... . . . . . . . . . . . . . . . . . 9

2.9 Procéder à la fermeture du mois . . . . . . . . . . . . . . . . . 10

2.7 vérifier le sommaire . . . . . . . . . . . . . . . . . . . . 10

2.8 Vérifier la saisie des sommaires . . . . . . . . . . . . . . . . 11 


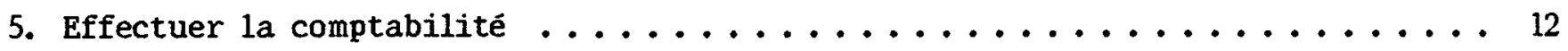

5.1 Sous-système "Enregistrer les ventes" . . . . . . . . . . . . . . . 12

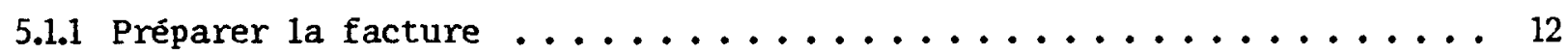

5.1.2 Dactylographier la facture ........................ 12

5.1.3 Reproduire les factures .......................... 13

5.1.4 Vérifier les copies de factures ..................... 13

5.1.5 Numéroter les copies de factures . . . . . . . . . . . . . . . . . 14

5.1.6 Additionner les copies de factures . . . . . . . . . . . . . . . . 14

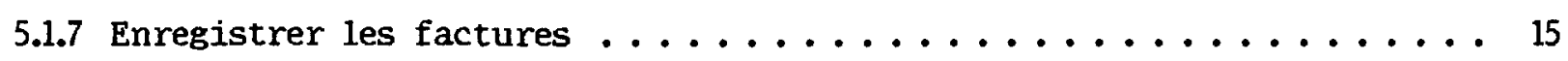

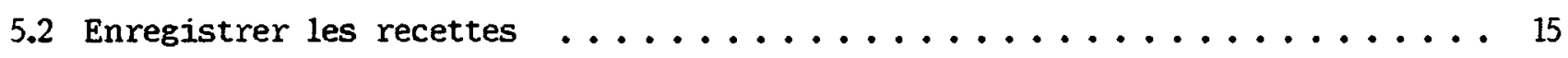

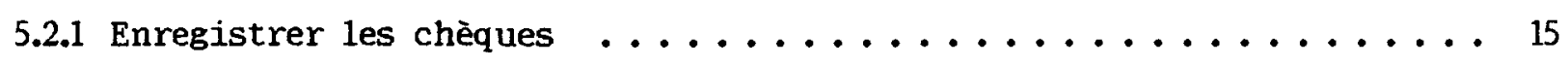

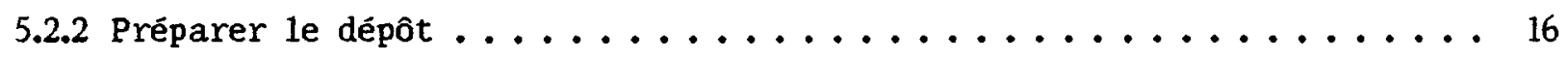

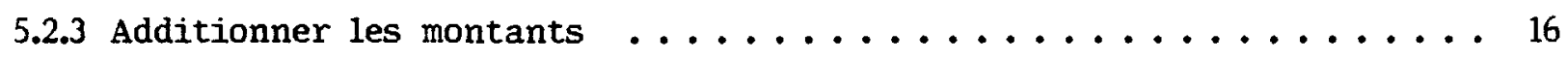

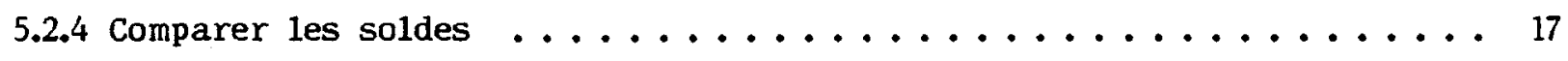

5.2.5 Enregistrer le numéro de facture .................... 17

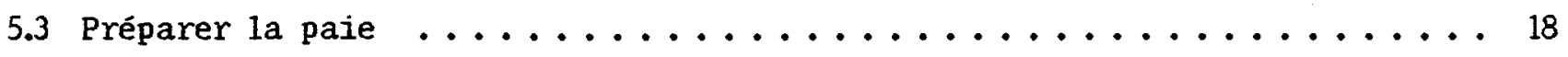

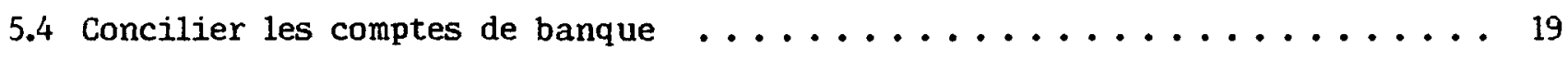

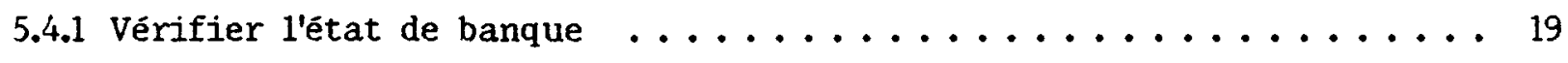

5.4.2 Identifier chèques et dépôts en circulation . . . . . . . . . . 19

5.4.3 Enregistrer les transactions bancaires ................. 20

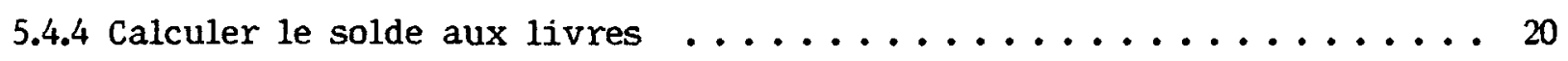

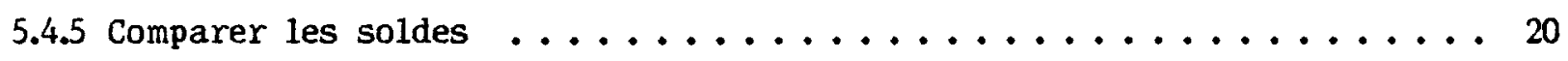

5.4.6 Compiler les transactions enregistrées .................. 21

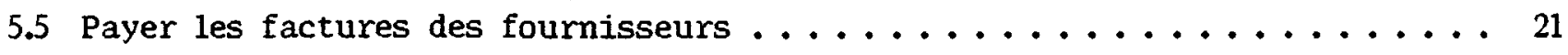

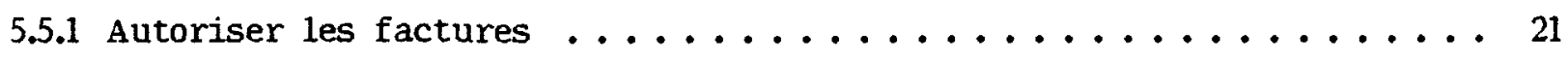

5.5.2 Sélectionner les factures à payer ....................... 21 
5.5 .3 Inscrire les codes d'imputation . . . . . . . . . . . . . . 22

5.5 .4 Préparer chèques et enveloppes . . . . . . . . . . . . . . . . 22

5.5 .5 Signer les chèques ............................ 23

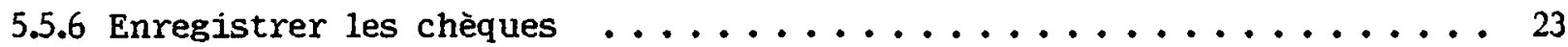

5.5.7 Faire un total de contrôle . . . . . . . . . . . . . . . . . . . 24

5.6 Produire les états financiers . . . . . . . . . . . . . . . . . . . . 24

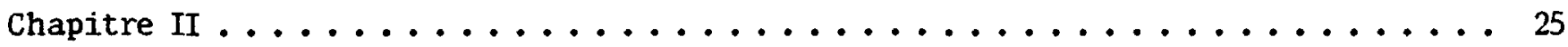

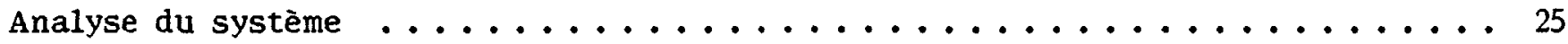

2. Analyse du processus "Compiler feuilles de temps" . . . . . . . . . . . . . . 25

2.1 Support utilisé pour la production des feuilles de temps . . . . . . . . 25

2.2 Nombre d'ordinateurs et d'imprimantes . . . . . . . . . . . . . . 25

2.3 Fermeture de la semaine ou du mois et impression de la feuille de temps . 25

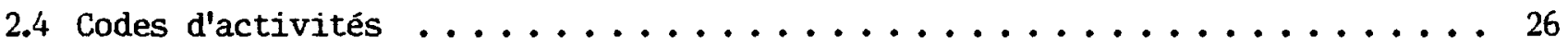

2.5 Saisie multiple ... . . . . . . . . . . . . . . . . . . . 27

2.6 Multitude de fichiers-clients . . . . . . . . . . . . . . . . . . 27

2.7 Atteinte des objectifs visés . . . . . . . . . . . . . . . . . . 27

5. Analyse du processus "Effectuer la comptabilité" ... . . . . . . . . . . 29

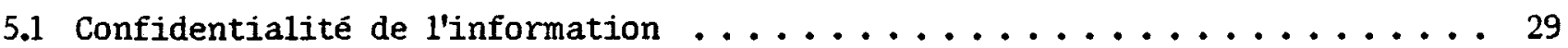

5.2 Mise à jour des auxiliaires de travaux en cours et de comptes à recevoir . 29

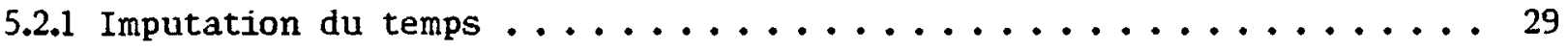

5.2.2 Rapport des travaux en cours ...................... 30

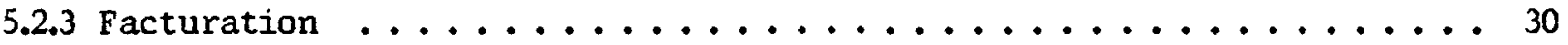


5.2.4 Enregistrement des recettes ......................... . . . 32

5.3 Conciliation bancaire ........................... 33

5.4 Payer les factures des fournisseurs ....................... 33

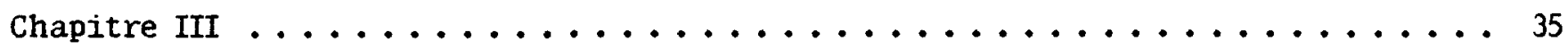

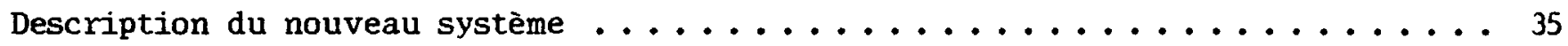

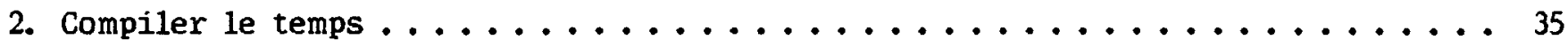

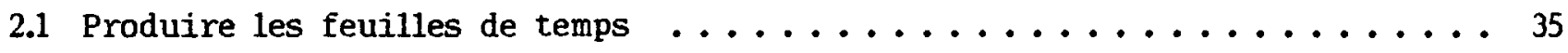

2.1.1 Enregistrer données feuilles - temps ................ 36

2.1.2 Imprimer feuille de temps ....................... 37

2.1.3 Corriger feuille de temps ...................... 37

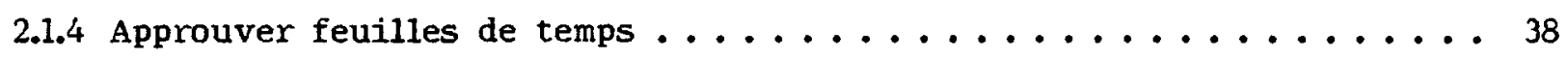

2.8 Saisir données feuilles de temps ....................... . . 38

2.8.1 Saisie de feuilles de temps produites manuellement . . . . . . . . 39

2.8.2 Saisie des feuilles de temps produites à l'aide de l'informatique . . . 40

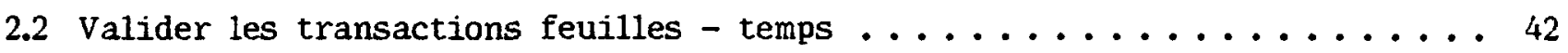

2.3 Corriger les transactions ........................... 42

2.3.1 Saisie de nouveaux clients ...................... 43

2.3.2 Modification d'une transaction .................... 43

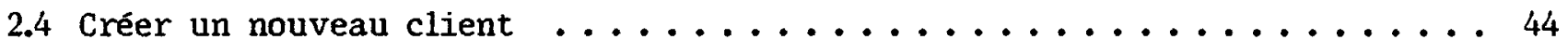

2.5 Imputer le temps ... . . . . . . . . . . . . . . . . . . . . 44

2.5 .1 Vérification de la validation ........................ 44

2.5.2 Vérification de la réception de toutes les feuilles de temps ...... 45

2.5.3 L'imputation des transactions ..................... 45 
2.6 Produire un rapport statistique $\ldots \ldots \ldots \ldots \ldots \ldots \ldots \ldots \ldots \ldots$

2.7 Contrôler feuilles de temps $\ldots \ldots \ldots \ldots \ldots \ldots \ldots \ldots \ldots \ldots$

2.7.1 Déterminer les feuilles manquantes $\ldots \ldots \ldots \ldots \ldots \ldots \ldots$. . . . . 47

2.7.2 Vérifier les feuilles manquantes $\ldots \ldots \ldots \ldots \ldots \ldots \ldots \ldots$

2.7.3 Calculer le temps supplémentaire $\ldots \ldots \ldots \ldots \ldots \ldots \ldots$

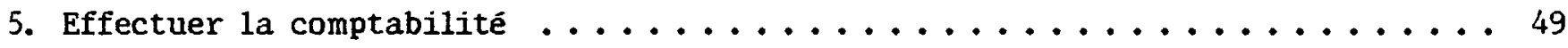

5.2 Facturer les clients ......................... 49

5.2 .1 Produire la facture $\ldots \ldots \ldots \ldots \ldots \ldots \ldots \ldots \ldots . \ldots \ldots$

5.2.2 Dactylographier la facture ................... 49

5.2 .3 Saisir la facture ....................... 50

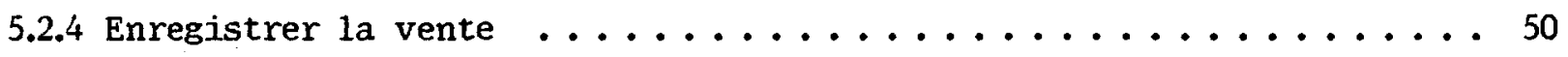

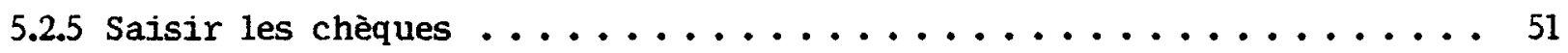

5.2.6 Enregistrer les recettes $\ldots \ldots \ldots \ldots \ldots \ldots \ldots \ldots \ldots \ldots \ldots \ldots$

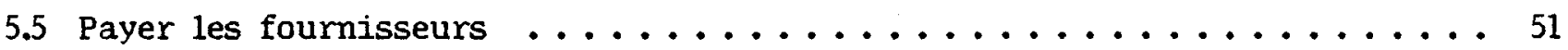

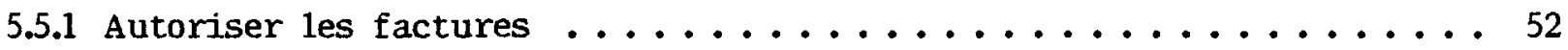

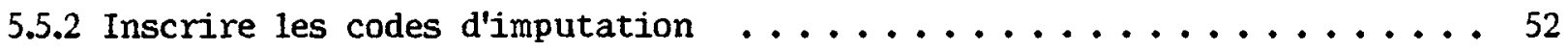

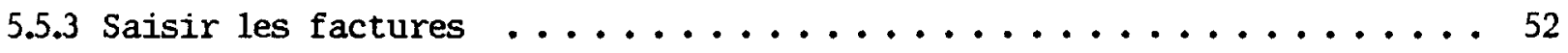

5.5.4 Imprimer la liste des factures à payer $\ldots \ldots \ldots \ldots \ldots \ldots$

5.5 .5 Retenir certaines factures $\ldots \ldots \ldots \ldots \ldots \ldots \ldots \ldots \ldots \ldots \ldots$

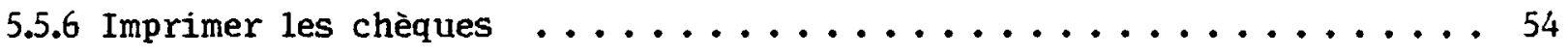

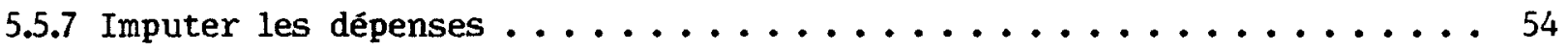

5.5.8 Enregistrer les nouveaux fournisseurs $\ldots \ldots \ldots \ldots \ldots \ldots$

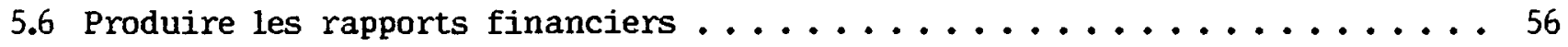

5.6 .1 Saisir les régularisations ................... 56

5.6.2 Enregistrer les écritures automatiques $\ldots \ldots \ldots \ldots \ldots \ldots$ 


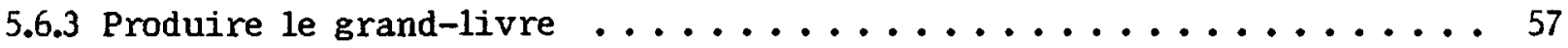

5.6.4 Produire le grand-livre cumulatif $\ldots \ldots \ldots \ldots \ldots$

5.6.5 Produire l'auxiliaire des travaux en cours. . . . . . . . . . . . 58

5.6.6 Produire l'auxiliaire des comptes à recevoir . . . . . . . . . . 5 59

5.6.7 Produire l'auxiliaire des comptes à payer . . . . . . . . . . . . 59

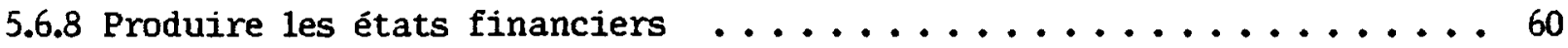


Préambule

Pour analyser le système d'information comptable des entreprises du Groupe Harel, Drouin, nous avons recenser l'ensemble des activités (1) composant le système d'information des entreprises du dit groupe. Suite à cette opération, nous avons attribué le numéro cinq (5) à l'activité concernant le traitement des données comptables des entreprises du Groupe Harel, Drouin et le numéro deux (2) à l'activité dont l'objectif est de compiler le temps travaillé à divers dossiers.

Le sujet de notre étude concerne donc la comptabilité et le traitement du temps. Nous avons inclus le traitement du temps dans notre étude, car le temps constitue la principale source de revenu des entreprises du Groupe Harel, Drouin.

1- production d'états financiers, fiscalité, administration, comptabilité, service d'information, marketing, etc. 
Introduction

Le présent document résulte d'une étude portant sur le système d'information comptable des entreprises du Groupe Harel, Drouin. Afin d'effectuer notre étude, nous avons employé la méthodologie des diagrammes de flux d'information. Cette méthodologie associée à des graphiques et des dictionnaires nous a permis d'identifier et de maitriser toutes les opérations composant le système d'information. Les diagrammes permettent d'identifier les activités et les données composant le système actuel. Ils permettent également la mise en évidence de toutes les forces et faiblesses du présent système et l'identification des besoins d'informations non comblés par le système actuel. Nous avons également utilisé cette méthodologie pour développer le nouveau système.

Ce document est donc composé de trois parties distinctes. La première partie décrit le système actuel. La seconde partie analyse le système et présente ses faiblesses. La dernière partie propose un nouveau système plus efficace qui élimine les faiblesses du premier. 


\section{Chapitre I}

Description du système actue1

\section{Présentation générale du système actuel}

Le système comptable des entreprises constituant le Groupe Harel, Drouin concerne les trois entités suivantes:

- Harel, Drouin et Associés

- Harel Drouin Gestion Conseil

- Les Services Harel Drouin

Les opérations comptables de ces entreprises sont très similaires. Le système comptable de la Société Harel Drouin est composé de différents journaux, d'un auxiliaire des travaux en cours, d'un auxiliaire des comptes à recevoir et d'un grand-livre. Il n'y a pas d'auxiliaire de comptes à payer. Le nombre de factures payées par la société ne justifie pas l'utilisation de cet auxiliaire. Actuellement, l'auxiliaire des travaux en cours est extra- comptable, c'est-à-dire que l'enregistrement de transactions dans cet auxiliaire ne modifie pas automatiquement le compte "travaux en cours" du grandlivre. Ce dernier doit être mis à jour par écriture de journal.

Le système comptable de la corporation Harel Drouin Gestion Conseil présente exactement les mêmes caractéristiques que la société mentionnée précédemment. I1 n'y a pas d'auxiliaire de comptes à payer même si la corporation effectue le paiement de ses factures. Le système comptable des Services Harel Drouin, une corporation de gestion, est constitué de différents journaux et d'un grand-livre. Cette corporation verse les salaires des employés du Groupe, paie les factures des fournisseurs de la Société et 
toutes les autres factures concernant les frais partagés (ex: papeterie) par les entreprises du Groupe. Elle a recours aux services de la Banque Royale pour le traitement des salaires. Il n'y a pas d'auxiliaire de comptes à payer. La gestion de ces comptes, le traitement et la production des chèques sont effectués manuellement. 
2. Compiler feuilles de temps

Afin de produire leurs feuilles de temps, le personnel utilise un progiciel créé à l'aide de "Symphony"(1). Ce progiciel est présenté sous forme de menu et composé de fenêtres, de bases de données et de macro-commandes. Pour utiliser ce progiciel, le personnel doit avoir à sa disposition un ordinateur sur lequel est installé symphony et une imprimante de marque "Epson." ou compatible "Epson".

Ce logiciel, qui produit un sommaire mensuel des activités par individu, a été créé dans le but de réduire les couts reliés à la saisie des feuilles de temps hebdomadaires.

\subsection{Enregistrer les données de base}

Les données de base servent à identifier l'auteur de la feuille de temps et à calculer le temps supplémentaire. Pour produire leur feuilles de temps, le personnel doit enregistrer les données de base. A la première utilisation du progiciel, les personnes inscrivent leur nom, leur numéro d'employé et le code de leur activité principale. Ces données sont enregistrées d'une façon permanente. A chaque semaine, les personnes doivent enregistrer la date de la feuille de temps et les heures régulières de la semaine pour laquelle elles produisent une feuille de temps.

2.2 Enregistrer données pour feuille de temps

A tous les jours, le personnel prend note de son emploi du temps dans son agenda. Pour enregistrer les données nécessaires à la production de la feuilles de temps,

1- marque de commerce d'un logiciel développé par Lotus Corporation. 
l'individu enregistre, sur un disque magnétique (communément appelé disquette) 1'information inscrite dans son agenda.

Avant d'expliquer le procédé utilisé pour enregistrer les données nécessaires à la production des feuilles de temps, nous devons vous signifier le sens de l'expression "fiche-activité". Les clients des entreprises du Groupe Hare1, Drouin sont identifiés pas un numéro. Les tâches appelées activités exécutées au cours d'une journée sont également identifiées par un numéro. Il y a des activités facturables et des activités non facturables. Une fiche-activité contient un code facturable et un numéro de client. Ainsi, si deux activités différentes sont exécutées pour un client, nous aurons deux fiches-activités.

Pour enregistrer les données nécessaires à la production des feuilles de temps, l'individu demande à l'ordinateur de trouver la fiche-activité désirée. Cette méthode est la plus employée. Lorsque l'usager désire utiliser cette méthode, le programme utilise une fiche spéciale que l'on nommera "fiche de recherche" dans laquelle l'usager enregistre son critère de recherche (les premières lettres du nom du client ou d'une activitê non facturable suivies d'une étoile).

Deux méthodes de recherche peuvent être utilisées: une méthode numérique et une méthode alphabétique. La méthode de recherche numérique peut être employée dans les cas d'activités facturables seulement, car elle s'effectue à l'aide du numéro de client. Les fiches-activités concernant des activités non facturables ne contiennent pas de numéro de client. Lorsque l'usager emploie cette méthode, l'ordinateur lui demande le numéro du client. 
La méthode de recherche alphabétique peut être employée pour les deux types d'activités, car elle procède à l'aide du nom du client (dans le cas d'activité facturable) ou du nom de l'activité.

L'ordinateur recherche la fiche-activité demandée. Lorsque l'ordinateur trouve plusieurs fiches correspondant au critère d'identification, il mentionne le nombre de fiches correspondant au critère fourni. L'usager sélectionne la fiche correspondant à l'activité désirée et enregistre le temps travaillé à côté de la journée correspondante. Lorsqu'il n'y a pas de fiche d'activité, l'usager doit créer la fiche à la fin de la base de données.

Fréquemment, par mégarde, l'usager crée une seconde "fiche de recherche" ou détruit la fiche de recherche. Dans le premier cas, le programme n'effectue plus adéquatement la recherche et mentionne que toutes les fiches correspondent au critère de recherche fourni. L'usager croit alors que logiciel ne fonctionne plus et demande l'aide du coordonnateur de l'informatique. Ce problème est réglể rapidement. Dans le second cas, le programme devient inutilisable. Ce problème peut être réglé de deux façons:

- la première, est de "sortir" du logiciel sans effectuer de sauvegarde et de recommencer le travail effectué.

- 1a seconde est de modifier la programmation du logiciel. Cela peut être fait seulement par le concepteur et engendre certains délais.

2.3 Imprimer la feuille de temps

Pour produire une feuille de temps, le progiciel imprime toutes les fichesactivités contenant un temps différent de zéro. 
Les fiches-activités dont le code d'activité correspond à une activité facturable (1) sont imprimées en premier. Ces fiches-activités sont suivies par la sommation du temps facturable pour chaque journée et pour la semaine. Ensuite, le progiciel imprime les fiches-activités dont le code d'activité correspond à une activité non facturable (2). Ces fiches-activités sont également suivies par la sommation du temps non facturable pour chaque journée et pour la semaine. Finalement, le progiciel imprime la sommation du temps facturable et non facturable, calcule le temps supplémentaire à l'aide de l'information enregistrée dans les données de base et imprime le résultat de ses calculs.

\subsection{Vérifier la feuille de temps}

Une fois que la feuille de temps est imprimée, la personne qui a enregistré l'information de la feuille de temps s'assure que toutes les données enregistrées sont exactes. Dans le cas où des erreurs s'y seraient glissées, elle corrige les données saisies. Pour effectuer les corrections, le procédé est le même que pour l'enregistrement. Dans le cas où il n'y a pas de correction, la feuille de temps est déposée sur le bureau du supérieur immédiat.

\subsection{Approuver 1a feuille de temps}

L'approbation de la feuille de temps s'effectue en vérifiant la répartition entre le temps facturable et le temps non facturable. Le temps imputé à chacun des clients

$1-$ code d'activité > $99 \neq 740$

2- code d'activité $<100$ ou $=740$ 
est également contralé. Quelquefois des modifications sont demandées. Lorsque la feuille de temps est approuvée, l'usager procède à la fermeture de la semaine.

\subsection{Procéder à la fermeture de la semaine}

La fermeture de la semaine consiste, pour chaque fiche d'activité, à additionner les heures inscrites, à reporter le résultat au bas de la fiche dans la semaine correspondante (lère semaine, $2^{\text {ème }}$ semaine, ... etc.) et à remettre en blanc les espaces qui avaient été utilisés pour inscrire les heures travaillées. Il arrive fréquemment que les usagers oublient de procéder à la fermeture de la semaine. Dans ce cas, lors de la production de la feuille de temps de la semaine suivante, les données de la semaine précédente sont additionnées avec celles de la semaine courante. L'usager peut remédier à ce problème de la manière suivante:

- effacer les données de la semaine courante

- procéder à la fermeture de la semaine précédente

- enregistrer à nouveau les données de la semaine courante.

Ce problème engendre une perte de temps plus ou moins longue qui varie en fonction de la quantité de données qui ont été saisies avant de constater l'erreur.

2.6 Produire le sommaire mensue1

Tout comme pour les feuille de temps, l'impression du sommaire consiste à imprimer toutes les fiches dont le total du mois est différent de zéro en faisant une distinction entre les activités facturables et non facturables. 
Le sommaire est présenté sous le même format que la feuille de temps. Cependant, nous retrouvons:

- des semaines à la place des journées

- des totaux pour chaque semaine au lieu des totaux pour chaque journée

I1 est fréquent de retrouver des informations erronées dans le sommaire mensuel. On rencontre fréquemment les erreurs suivantes:

- $\quad$ le temps supplémentaire est inexact

- la sommation des heures est erronée

- les heures travaillées au cours d'une semaine n'apparaissent pas dans le sommaire.

\subsection{Procéder à la fermeture du mois}

Une fois que la dernière semaine du mois a été fermée, l'usager peut fermer le mois. Le principe est le même que pour la fermeture de la semaine. Pour toutes les fiches qui ont un code d'activité inférieur à 100 ou égal à 740 , on additionne les heures inscrites à la lère, 2ème, Jème, 4ème et 5ème semaine de chaque fiche et on reporte le résultat dans la fiche des statistiques annuelles dans la section du temps non facturable pour le mois correspondant. Le même processus s'applique pour les fiches dont le code d'activité est supérieur à 99 et différent de 740 .

\subsection{Vérifier le sommaire}

Lorsque tous les sommaires sont imprimés et approuvés par le supérieur immédiat, ces derniers sont remis à la réceptionniste. Celle-ci s'assure que tous les sommaires 
soient remis et qu'il n'y manque aucun numéro de client. Lorsque qu'il manque des numéros de clients, elle consulte la liste des clients aux travaux en cours pour retracer le numéro du client et l'inscrit sur le sommaire.

Occasionnellement, le client n'est pas retrouvé dans la liste. Il s'agit donc d'un nouveau client. Pour que les coordonnées du client soient inscrites dans la prochaine liste de clients aux travaux en cours, la réceptionniste complète une fiche nommée "Fiche client". Elle y inscrit le nom du client et le numéro qui lui sera attribué. Manuellement, elle inscrit le nom du client et le numéro sur la liste des clients aux travaux en cours.

Dans la liste des clients aux travaux en cours, ceux-ci y apparaissent dans l'ordre alphabétique. Pour attribuer un numéro de client, la réceptionniste identifie l'endroit où il sera situé dans la liste. Le numéro du client sera donc supérieur à celui qui le précède et inférieur à celui qui le suivra.

\subsection{Vérifier la saisie des sommaires}

Les sommaires reviennent de 1a "Société d'Informatique de Gestion" accompagnés d'un rapport nommé "validation des feuilles de temps". Ce rapport contient des messages d'erreurs qui proviennent de la validation des données saisies. Chaque message est analysé afin de vérifier s'il y a lieu de procéder à des corrections. Les demandes de correction sont acheminées à la "Société d'Informatique de Gestion". 
5. Effectuer la comptabilité

5.1 Sous-système "Enregistrer les ventes"

\subsubsection{Préparer la facture}

Pour préparer une facture, l'associé ou le vérificateur senior consulte l'auxiliaire des travaux en cours pour connaître les montants imputés dans le compte du client. Etant donné que les dernières données ont été enregistrées à l'auxiliaire des travaux le $30 \mathrm{du}$ mois précédent, l'auxiliaire, au moment de la facturation, n'inclut pas le temps travaillé sur le dossier du client après le 30. Pour connaitre le temps travaillé sur le dossier d'un client entre le 30 du mois précédant et la date de la facturation, l'associé ou le vérificateur senior, consulte les personnes de son équipe afin de connaitre le nombre d'heures travaillées sur le dossier après le 30.

A partir de ces informations, l'associé ou le vérificateur senior prépare la facture en dictant le contenu sur dictaphone, en modifiant une copie de la facture antérieure ou en la rédigeant.

\subsubsection{Dactylographier la facture}

Dans le cas d'un ancien client, la secrétaire récupère, sur son appareil de traitement de texte, 1a facture antérieure et effectue les modifications nécessaires. 


\subsubsection{Reproduire les factures}

La secrétaire qui a dactylographié les factures en effectue différentes copies. Les secrétaires de Harel, Drouin et Associés reproduisent des factures sur du papier vert et sur du papier blanc. Les secrétaires de Harel, Drouin Gestion Conseil reproduisent les factures de Pierre-Paul Morin sur du papier rose, celles de Gérald Bélanger sur du papier jaune et celle de Jean-Guy Gernier sur du papier bleu. Elles reproduisent également les factures sur du papier blanc. Les copies vertes, jaunes, bleues et roses vont au département de comptabilité. Les copies blanches de Harel, Drouin \& Associés sont classées dans le dossier de correspondance et, dans certain cas, une autre copie blanche est broché sur la couverture du même dossier. Les copies blanches de Harel Drouin Gestion Conseil sont classées dans le dossier du mandat (cas de Pierre-Paul Morin) ou dans dossier des comptes à recevoir (cas de Gérald Bélanger et de Jean-Guy Grenier).

\subsubsection{Vérifier les copies de factures}

Au département de comptabilité, pour chacune des factures, le montant est mis en évidence. Pour les factures n'ayant qu'une seule page, le montant est encerclé. Dans le cas des factures de plus d'une page, le montant qui apparaît généralement sur la dernière page, est reporté sur la première page. Le montant indiqué sur la dernière page est rayé afin d'éviter toute confusion. 


\subsubsection{Numéroter les copies de factures}

Le département de comptabilité numérote les copies des factures à l'aide de deux numéroteurs (un pour les copies vertes de Harel, Drouin et Associés, l'autre pour les copies roses, jaunes et bleues de Harel Drouin Gestion Conseil).

\subsubsection{Additionner les copies de factures}

Ce processus comprend cinq additions, c'est-à-dire une addition par groupe de factures ${ }^{(1)}$. La sommation des copies vertes des factures acceptées par le système informatique est enregistrée dans le "journal des ventes temporaire" lors de l'opération.

La sommation des copies vertes des factures refusées est inscrite sur un formulaire de transaction. Il en est de même pour les copies jaunes, roses et bleues des factures de Harel Drouin Gestion Conseil. Les copies vertes des factures refusées, les copies roses, jaunes et bleues, accompagnées de formulaires de transactions sont envoyées à la Société d'informatique de gestion. Les employés de 1a Société d'informatique de gestion procéderont à l'enregistrement de ces factures.

1- - Le premier groupe est composé des copies vertes acceptées par le système informatique.

- Le deuxième groupe est composé des copies vertes refusées par le système informatique.

- Le troisième groupe est composé des copies roses.

- Le quatrième est composé des copies jaunes.

- Le cinquième est composé des copies bleues. 


\subsubsection{Enregistrer les factures}

Une personne du département de comptabilité va au bureau de la Société d'informatique de gestion pour enregistrer les factures de Harel Drouin et Associés (copies vertes). Il arrive fréquemment qu'à cause d'une défectuosité du logiciel utilisé, le système refuse l'enregistrement d'une facture en mentionnant que le client n'existe pas. Pourtant le nom et le numéro du client refusé apparaissent dans la "liste des clients au travaux en cours".

L'enregistrement des factures de Harel, Drouin et Associés (copies vertes) génère donc deux groupes de factures; un groupe représentant les factures acceptées par le système informatique et un second représentant les factures refusées. L'information inscrite sur les factures du premier groupe est enregistrée dans un journal nommé par la Société d'informatique de gestion "Journal des ventes temporaire".

\subsection{Enregistrer les recettes}

\subsubsection{Enregistrer les chèques}

Lorsque nous recevons des chèques de nos clients, nous enregistrons ces derniers sur un formulaire de transaction. Sur ce formulaire, nous inscrivons le numéro du client que nous avons retracer sur la liste de clients aux travaux en cours, le nom du client et le montant du chèque. L'information relative aux chèques reçus est également enregistrée dans un dossier que nous avons nommé "dossier comptes à recevoir"(1). Dans

1- Ce dossier est utilisé par Jean-Guy Grenier et Gérald Bélanger de Hare1, Drouin Gestion-Conseil. 
ce dossier, nous retrouvons une copie des factures qui sont envoyées aux clients. Lorsque les clients de monsieur Gérald Bélanger paient leurs factures, sa secrétaire, à l'aide du chèque, indique la mention "payée" sur la facture correspondante. Dans le cas de monsieur Grenier, sa secrétaire consulte les formulaires de transaction, afin de prendre connaissance des chèques reçus. Par la suite, pour chacun des chèques reçus, elle note sur son sommaire de comptes à recevoir la date et le numéro du chèque.

Lorsque nous ne pouvons identifier le client à cause de l'illisibilité de la signature, nous appelons à la banque émettrice pour connaitre le nom du signataire.

\subsubsection{Préparer le dépôt}

Pour préparer le dépôt, nous additionnons tous les montants des chèques reçus. Le résultat de la sommation est inscrite sur le bordereau de dépôt avec la date. Le tout est envoyé à la banque.

\subsubsection{Additionner les montants}

Nous additionnons tous les montants des chèques qui ont été inscrits sur le formulaire de transaction. Le résultat de l'addition est reporté sur le bas du formulaire de transactions. Par la suite, sur une calculatrice à ruban, nous additionnons les montants inscrits au bas du formulaire. Le ruban produit est joint aux formulaires de transactions et le tout est acheminé à la Société d'informatique de gestion. 


\subsubsection{Comparer les soldes}

Une fois que le dépôt a été préparé et que tous les formulaires ont été additionnés, nous comparons les résultats. Le montant du dépôt et la sommation des formulaires de transactions doivent être égaux. Dans le cas contraire, lorsqu'une erreur s'est glissée, nous devons vérifier le montant du dépôt et les additions effectuées.

\subsubsection{Enregistrer le numéro de facture}

Lorsque nous enregistrons des chèques sur un formulaire de transactions, nous inscrivons comme référence le numéro des factures qui ont été envoyées aux clients. La méthode utilisée pour retracer le numéro de facture est différente selon le type de facture. Pour les factures de Harel, Drouin et Associés, nous allons à la Société d'informatique de gestion consulter, à l'aide de l'écran, nos fichiers de comptes à recevoir. Occasionnellement, il arrive que certaines factures ne s'y trouvent pas, car elles avaient été refusées par le système lors de la saisie. Quant nous identifions une facture ayant le même montant que le chèque, le numéro de référence de la facture est écrit sur le formulaire de transactions. S'il n'y a pas de facture ayant le même montant que le chèque, il est possible que le chèque reçu corresponde à une facture qui avait été rejetée par le système. Pour trouver le numéro de référence, nous consultons le rapport intitulé "liste des comptes à recevoir". Si la facture n'y apparất pas, le chèque envoyé par le client correspond probablement à une avance sur des honoraires. Dans ce cas, le numéro "00000" est employé à titre de référence.

Pour les factures de Harel Drouin Gestion Conseil, les numéros de factures sont retracés dans le rapport intitulé "liste des comptes à recevoir. cause du nombre 
restreint de factures, le procédé permettant de consulter les comptes à recevoir n'a pas été développé.

\subsection{Préparer la paie}

Actuellement, nous avons recours aux services de la Banque Royale afin de produire la paie. Cette dernière calcule la paie de chacun des employés, émet le talon de paie, dépose le montant net de la paie dans le compte bancaire de chaque employé et nous fournit différents rapports nous indiquant les écritures à enregistrer dans nos registres comptables.

Étant donné:

- qu'il n'y a aucune variation dans le salaire hebdomadaire pour la très grande majorité des employés;

- que le temps supplémentaire n'est rémunéré qu'une fois par année;

- que la préparation des données nécessaires à la production de la paie ne nécessite au maximum qu'une demie-journée de travail.

Il est donc pratiquement impossible de réduire le cout et le temps nécessaires à la production de la paie. cause des dépôts bancaires, les employés n'ont pas besoin de se rendre au bureau pour récupérer leur chèque de paie. 


\subsection{Concilier les comptes de banque}

\subsubsection{Vérifier l'état de banque}

Cette opération consiste à vérifier l'exactitude de toutes les transactions ayant modifié les comptes bancaires. Une comparaison est effectuée entre tous les chèques remis par la banque et les montants inscrits sur les états de banque. Nous procédons de la même façon avec les dépôts. Rarement, des erreurs sont identifiées. Dans ces cas, elles sont signalées à la banque afin que cette dernière effectue des corrections. Une fois que toutes les transactions aient été vérifiées, les chèques sont classés en ordre numérique.

\subsubsection{Identifier chèques et dépôts en circulation}

Pour identifier les chèques et les dépôts en circulation, on regarde si les chèques enregistrés dans la conciliation bancaire du mois précédent ont été retournés par la banque. Tous les chèques enregistrés sur la conciliation bancaire du mois précédent qui n'ont pas été retournés par la banque sont inscrits sur la conciliation bancaire du mois courant. Par la suite, nous prenons l'ensemble des copies jaunes et nous regardons, pour chaque copie jaune, si le chèque a été retourné par la banque. L'information contenue sur toutes les copies jaunes, pour lesquelles nous n'avons pas reçu le chèque, est inscrite sur la conciliation bancaire du mois courant.

La procédure est la même pour les dépôts en circulation. Tous les dépôts en circulation apparaissant sur la conciliation bancaire du mois précédent, qui ne figurent pas sur l'état de banque, sont reportés sur la conciliation bancaire du mois 
courant. Il en est de même pour tous les dépôts du mois courant, qui n'apparaissent pas sur l'état de banque.

\subsubsection{Enregistrer les transactions bancaires}

Par "transactions bancaires", nous faisons référence à toutes les transactions autres que les chèques et dépôts qui ont modifié le solde du compte. Ces transactions sont enregistrées sur la conciliation bancaire du mois courant, dans le livre utilisé pour le suivi bancaire et sur un formulaire de transaction, formulaire qui sera utilisé par S.I.G. pour mettre à jour les livres comptables.

\subsubsection{Calculer le solde aux livres}

Le solde aux livres apparaissant sur la conciliation bancaire du mois courant est obtenu à partir du solde aux livres de la conciliation du mois précédent auquel nous avons soustrait les chèques et ajouté les dépôts du mois courant. Le solde ainsi obtenu doit être le même que celui qui figure dans le livre utilisé pour le suivi bancaire (5.4.5 comparer les soldes).

\subsubsection{Comparer les soldes}

La dénomination de cette activité regroupe deux processus semblables effectués avec des données différentes. Ainsi, le premier processus consiste à comparer le solde obtenu lors de l'opération "calculer le solde aux livres" avec le solde inscrit dans le livre utilisé pour le suivi bancaire. 
Le second processus consiste à comparer le solde aux livres redressé de la conciliation bancaire du mois courant au livre utilisé pour le suivi bancaire après l'enregistrement des transactions bancaires (autres que les chèques et dépôts).

\subsubsection{Compiler les transactions enregistrées}

Cette opération consiste à additionner ou soustraire, selon le cas, les transactions enregistrées sur la conciliation bancaire et à inscrire, sur la conciliation, les montants obtenus. Elle consiste également à additionner ou soustraire, selon le cas, les transactions enregistrées dans le livre de banque afin d'obtenir le nouveau solde.

\subsection{Payer les factures des fournisseurs}

\subsubsection{Autoriser les factures}

Pour autoriser une facture, un associé y inscrit ses initiales ou sa signature. Pour être payées, toutes les factures doivent être autorisées à l'exception des factures concernant des paiements mensuels.

\subsubsection{Sélectionner les factures à payer}

A toutes les semaines, pour chaque fournisseur, on retire du lots de factures autorisées toutes les factures qui doivent être payées, c'est-à-dire celles qui sont datées d'environ trente jours. 


\subsubsection{Inscrire les codes d'imputation}

Les codes d'imputation sont les numéros des comptes du grand-livre dans lesquels sera enregistrée chaque facture. Certaines factures peuvent avoir plus d'un numéro de compte.

\subsubsection{Préparer chèques et enveloppes}

Après avoir inscrits les codes d'imputation sur les factures, les chèques sont préparés. Sur les chèques, nous inscrivons:

- la date du chèque

- le nom du fournisseur

- le montant du chèque

Sur le talon du chèque, nous inscrivons:

- la date du chèque

- le ou les codes d'imputation et le montant correspondant

- le nom du fournisseur

Le chèque et le talon sont reproduits en deux copies: une blanche et une jaune. Le talon du chèque n'est pas conservé.

Lorsqu'il y a un numéro de client inscrit sur la facture, nous l'enregistrons avec le montant et la référence sur une feuille de déboursés lesquels sont facturables. Les employés de la Société d'informatique de gestion utilisent cette feuille pour enregistrer 
les déboursés engagés par la société Harel, Drouin dans l'auxiliaire des travaux en cours.

\subsubsection{Signer les chèques}

Le directeur administratif (monsieur Réal Brière) et l'associé responsable de l'administration (monsieur Gilles Drouin) sont les seules personnes autorisées à signer les chèques. Le directeur administratif appose sa signature en premier lieu. Cependant, avant de signer un chèque, ce dernier s'assure que la personne qui a autorisé le paiement de la facture est en droit de le faire. Par la suite, il vérifie:

- l'exactitude du code d'imputation

- le bénéficiaire

- la date du chèque

Ensuite, les chèques sont remis à l'associé responsable de l'administration qui, après avoir effectué une vérification sommaire des factures, y appose sa signature.

Lorsque les chèques sont signés, nous séparons les chèques des copies. Nous postons les chèques et les copies de factures, nommées pièces, aux fournisseurs. Les copies et les factures concernées par les chèques sont reliées ensembles.

\subsubsection{Enregistrer les chèques}

Afin d'avoir un suivi du solde des comptes bancaires, l'information contenue sur les copies jaunes est enregistrée dans le livre nommé "livre de banque" (voir DFI, D5/1) de la corporation ou de la société émettrice du chèque. 
5.5.7 Faire un total de contrôle

Pour obtenir un total de contrôle, on additionne, à l'aide d'une calculatrice à ruban, tous les montants de chèques inscrits sur la copie jaune. On joint le ruban produit aux copies jaunes. Le tout est envoyé à la Société d'informatique de gestion.

\subsection{Produire les états financiers}

Au début de notre étude, nous avions pensé que la production des états financiers constituaient une activité inhérente au système d'information. Cela est plus ou moins exact.

La Société d'informatique de gestion produit des états financiers mensuels. Ces états sont incomplets, car ils ne comprennent pas les comptes à payer, les travaux en cours et les revenus. Ils sont donc d'aucune utilité.

Par contre, à chaque trimestre, à partir de la balance de vérification fournie par la Société d'informatique de gestion, des états financiers sont créés et chaque poste de ceux-ci est analysé.

cause de la stricte confidentialité de l'information, nous avons été dans l'impossibilitê de décrire et d'analyser cette activité. 


\section{Chapitre II}

Analyse du système

2. Analyse du processus "Compiler feuilles de temps"

2.1 Support utilisé pour la production des feuilles de temps

Les feuilles de temps sont produites à l'aide du logiciel symphony. L'utilisation de ce logiciel présente quelques problèmes, car il est très facile de modifier volontairement ou par inadvertance la programmation du logiciel. Cela a pour conséquence de causer des erreurs en cours d'exécution, de produire des feuilles de temps ou des sommaires contenant de l'information erronée.

\subsection{Nombre d'ordinateurs et d'imprimantes}

A la fin du mois, une grande partie du personnel désire produire leurs feuilles de temps. Cette situation provoque un goulot d'étranglement, puisque le nombre d'ordinateurs et d'imprimantes "Epson" est insuffisant pour faire face à la demande. Cette situation peut être facilement améliorée en suggérant au personnel de produire la feuille de temps dès la fin de la semaine.

2.3 Fermeture de la semaine ou du mois et impression de la feuille de temps

Tel que conçu actuellement, le programme ne permet pas de modifier le contenu d'une feuille de temps après la fermeture de la semaine. Dans le cas où des corrections 
sont nécessaires, elles sont effectuées manuellement sur la feuille de temps et ne sont pas incluses dans le sommaire mensuel. Le sommaire doit également être corrigé.

Il est également assez fréquent que les gens "ferment" plus d'une fois la même semaine. Dans ce cas, le temps consacré à chaque activité de cette semaine est égale à zéro; le contenu de chaque journée ayant été mis à zéro lors de la première fermeture.

Pour corriger cette situation, la personne doit, à partir d'une disquette vierge, enregistrer à nouveau toutes les données de ses feuilles de temps du mois (cela implique l'enregistrement des noms et des numéros de clients qui avaient été enregistrés jusqu'à ce moment-là). L'autre solution est de corriger manuellement le sommaire. Dans un cas comme dans l'autre, les objectifs d'automatisation et d'économie ne sont pas atteints.

\subsection{Codes d'activités}

Le personnel de Harel Drouin et Associés et celui de Harel Drouin Gestion-Conseils utilisent la même liste de code d'activités (voir D 2/4). Cette 1iste de code d'activités comprend principalement des activités reliées au domaine de la vérification et de l'expertise comptable.

Les activités du personnel de Harel Drouin Gestion-Conseils diffèrent grandement des activités du personnel de Harel Drouin et Associés. Ainsi, le personnel de Harel Drouin Gestion-Conseils impute l'ensemble de leurs mandats sous les codes d'activités 500 (spécial) et 990 (divers). Harel Drouin Gestion-Conseils ne peut analyser la répartition du temps ou la facturation par activité. De plus, cette situation rend plus 
difficile la facturation et l'identification des travaux effectués à l'intérieur de chacun des mandats.

\subsection{Saisie multiple}

L'enregistrement des données pour la feuille de temps (voir processus 2.2) implique des recherches de fiche-activité. Lorsqu'une fiche-activité n'existe pas, nous devons créer la nouvelle fiche. Si plusieurs personnes ont travaillé sur le dossier d'un nouveau client, toutes ces personnes doivent enregistrer les informations nécessaires à la création de la nouvelle fiche dans leur banque de données.

Les sommaires mensuels sont envoyés chez la Société d'informatique de gestion afin que son personnel saisissent les données contenues dans 1e sommaire. Ces données seront utilisées pour mettre à jour l'auxiliaire des travaux en cours.

\subsection{Multitude de fichiers-clients}

Nous pouvons considérer l'ensemble des fiches dont le code d'activités est plus grand que 99 est différent de 740 comme étant un fichier client. Nous avons donc autant de fichiers-clients que de personnes qui produisent des feuilles de temps.

\subsection{Atteinte des objectifs visés}

Etant donné les nombreux problèmes rencontrés par les personnes lors de la production des feuilles de temps, le manque de souplesse du logiciel, les corrections 
effectuées manuellement et le temps que les gens prennent à produire leurs feuille de temps, il est évident que cette manière de procéder ne permet pas d'effectuer de réelles économies.

La façon la plus efficace de produire les feuilles de temps serait d'intégrer cette production à la mise à jour de l'auxiliaire des travaux en cours et ce, sans intervention humaine. Ainsi, nous serions assurés de l'exactitude des numéros et noms de clients, des codes d'activités et du temps imputé. De plus, nous éviterions tous les couts reliés à la saisie des sommaires. 
5. Analyse du processus "Effectuer la comptabilité"

5.1 Confidentialité de l'information

Tel que conçu actuellement, le système nécessite l'intervention des employés de la Société d'informatique de gestion afin de mettre à jour certains livres comptables. Cette situation ne nous permet pas de limiter physiquement et logiquement l'accès aux données comptables et n'assure pas la confidentialité de l'information.

5.2 Mise à jour des auxiliaires de travaux en cours et de comptes à recevoir

\subsubsection{Imputation du temps}

Au niveau de la mise à jour des travaux en cours, nous pouvons identifier plusieurs inefficacités. Dans un premier temps, nous constatons que la Société d'informatique de gestion effectue la saisie des sommaires mensuels, lesquels sont produits à partir d'un logiciel. Il y a donc une double saisie: saisie des données nécessaires à la production des sommaires et saisie des sommaires. Nous pourrions économiser les conts reliés à la saisie du sommaire si nous pouvions utiliser directement les données déjà enregistrées afin de mettre à jour les différents auxiliaires des travaux en cours. Cela nous permettrait également d'éliminer les délais qu'il y a présentement entre la production des feuilles de temps (1e 30 du mois) et la production des rapports reliés aux travaux en cours (vers le 12 du mois suivant). 


\subsubsection{Rapport des travaux en cours}

Nous constatons également que le rapport des travaux en cours, tel que présenté actuellement, et les codes d'activités employés ne répondent pas aux besoins de Harel Drouin Gestion Conseil. La liste des codes actuellement utilisée contient principalement des codes d'activités facturables reliés au domaine de l'expertise comptable. Les associés et employés de Harel Drouin Gestion Conseil imputent donc l'ensemble de leurs activités sous les codes 500 (spécial) et 990 (divers). En conséquence, le rapport de l'auxiliaire des travaux en cours ne peut être utilisé pour la facturation, car ce dernier fournit, pour chaque client et pour chaque employé ayant travaillé sur le dossier du client, le cumulatif hebdomadaire des heures travaillées sous le même code d'activités. Pour palier à cette situation, durant le cours de notre étude, le personnel de Harel Drouin Gestion Conseil a mis sur pied un système parallèle de travaux en cours. Nous avons donc des frais d'administration ou de gestion qui pourraient être éliminés avec un système d'information répondant aux besoins des différentes entités. Ce système devrait prévoir des codes d'activités reliés aux différents types de mandats exécutés par Harel Drouin Gestion Conseil, un emplacement nous permettant d'inscrire la description du travail effectué et la possibilité d'obtenir un rapport détaillé énumérant toutes les transactions ayant modifié l'auxiliaire des travaux en cours.

\subsubsection{Facturation}

L'enregistrement des factures présente également plusieurs problèmes. La méthodologie employée pour enregistrer les comptes à recevoir varie selon qu'il s'agit de factures d'Harel Drouin et Associés ou d'Harel Drouin Gestion Conseil. Les factures d'Harel Drouin et Associés sont saisies à l'écran (voir processus 5.1.1 Enregistrer les 
ventes). Cependant, il arrive que le système informatique refuse 1a saisie d'une facture en alléguant l'inexistence du client. Les factures refusées et les factures d'Harel Drouin Gestion Conseil sont remises aux employés de la Société d'informatique de gestion qui procèdent à la saisie. Le temps employé à effectuer la saisie de factures qui sont refusées par le système informatique et le temps du déplacement de l'employé de la Société Harel, Drouin aux bureaux de la Société d'informatique de gestion représentent des frais qui pourraient être épargnés.

Le second problème concerne le journal des ventes. Ce dernier contient seulement les factures saisies à l'écran et acceptées par le système informatique. Nous pouvons affirmer que ce journal nous fournit de l'information erronée, car il ne contient pas toutes les factures modifiant les auxiliaires des travaux en cours et des comptes à recevoir. Cette situation a comme conséquence d'alourdir le système d'enregistrement des recettes (5.2.4 enregistrement des recettes).

Nous augmenterons l'efficacité de nos employés et la qualité de notre information en utilisant un système qui permettrait la saisie des factures à nos bureaux, de retrouver dans le journal des ventes toutes les transactions ayant modifié les auxiliaires des travaux en cours et des comptes à recevoir.

Un système efficient devrait nous permettre, à partir de l'information contenue dans l'auxiliaire des travaux en cours, de produire des factures à l'écran et d'enregistrer celles-ci dans le journal des ventes. Il devrait permettre également la saisie de factures produites manuellement. La mise à jour des auxiliaires devrait être effectuée suite à une commande fournie par l'usager et ce, à partir du journal des ventes. Un tel 
système nous permettrait d'économiser presque tous les frais reliés à la dactylographie des factures et ceux reliés à la saisie de ces factures.

\subsubsection{Enregistrement des recettes}

Le principal problème relié à l'enregistrement des recettes réside au niveau des différents procédés employés pour identifier les factures pour lesquelles des chèques ont été reçus.

Pour les factures de Harel Drouin Gestion Conseil, le numéro de facture est identifié en consultant la liste des comptes à recevoir. Actuellement, la liste n'étant pas volumineuse, cette méthode ne présente aucun problème. Cependant, avec les années et une augmentation des clients, cette façon de rechercher un numéro de facture risque de devenir fastidieuse.

Dans le cas des factures de Harel Drouin et Associés, les numéros de factures sont généralement retracés à l'écran en consultant les comptes à recevoir. Cependant, il arrive qu'il est impossible de retracer les numéros de factures à l'écran. Dans ces cas, il s'agit d'avances sur honoraires ou bien, de factures qui avaient été refusées par le système informatique. (voir processus 5.1.6) Dans le cas des factures refusées, les numéros de factures sont retrouvés dans la liste des comptes à recevoir, liste volumineuse et difficile à manipuler.

Un système efficace devrait permettre la consultation de tous les comptes à recevoir à l'écran. Cela permettrait l'identification rapide des numéros de factures. Un système avec "code de sécurité" permettrait aux secrétaires de consulter les comptes 
à recevoir de leur patron et éliminerait leur coots reliés au maintient de systèmes parallèles. (voir processus 5.1.3) Le système devrait nous permettre d'obtenir la liste de toutes les factures dont le montant correspond au montant du chèque reçu.

\subsection{Conciliation bancaire}

Au niveau des conciliations bancaires, nous constatons qu'il y a une perte de temps au niveau de l'enregistrement des transactions bancaires, puisque ces dernières sont enregistrées deux fois: la première consiste à enregistrer ces transactions sur un "formulaire de transaction" qui est envoyé chez la Société d'informatique de gestion pour mettre à jour les livres comptables et la seconde consiste à enregistrer ces transactions dans le livre utilisé pour le suivi bancaire.

Un système efficace nous permettrait d'enregistrer les transactions bancaires dans le compte au grand-livre correspondant et éliminerait les livres de "suivi bancaire". Les administrateurs des entreprises du Groupe Harel, Drouin pourraient connaitre plus rapidement la situation réelle de l'encaisse.

\subsection{Payer les factures des fournisseurs}

Dans notre étude, nous avons identifié plusieurs tâches qui seraient éliminées avec l'amélioration du système d'information.

Actuellement, tous les chèques sont produits manuellement et l'information relative à ces chèques est inscrite par nos employés dans le livre du suivi bancaire et saisie par les employés de la Société d'informatique de gestion afin de mettre à jour les livres 
comptables. La production des chèques représente environ deux journées de travail et l'enregistrement de l'information dans le livre du suivi bancaire, une demie journée de travail.

L'utilisation d'un système informatique intégrant un auxiliaire des comptes à payer nous permettrait d'économiser deux journées de travail en produisant électroniquement les chèques. De plus, cela nous assurerait une meilleure gestion de nos comptes à payer. L'utilisation d'enveloppes à fenêtre nous permettrait d'éliminer la dactylographie des adresses des fournisseurs sur les enveloppes. La saisie de 1'information relative aux chèques dans le livre servant au suivi bancaire ne serait plus nécessaire et la saisie de l'information par les employés de la Société d'informatique de gestion serait remplacée par l'enregistrement des factures.

Le système informatique utilisé devra permettre la consultation des comptes à payer à l'écran et l'entrée des chèques produits manuellement. 


\section{Chapitre III}

Déscription du nouveau système

\section{Compiler le temps}

Ce processus contient des activités que nous pouvons qualifier de manuelles, des activités effectuées sur micro-ordinateurs et des activités qui seront effectuées sur mini-ordinateur. Tout le processus repose sur le principe que les feuilles de temps sont produites hebdomadairement.

Étant donné que nous ne pouvons élaborer un système d'information qui assumera entièrement tous les cas d'exceptions (1a fréquence de ces exceptions ne justifiant pas les couts rattachés à l'élaboration d'un système les traitant automatiquement), des procédés manuels devront quand même être exécutés afin d'intégrer ces cas au système global. Par exemple, si un associé désire effectuer une mise à jour de ses travaux en cours au milieu d'une semaine, le temps associé aux journées qui suivent la mise à jour devra être égal à 0 . Le travail exécuté pendant ces journées devra être enregistré la semaine suivante et être considéré comme du temps supplémentaire.

\subsection{Produire les feuilles de temps}

Avant de décrire le sous-processus 2.1 Produire les feuilles de temps, nous désirons mentionner que ce processus n'a aucune relation physique avec le système informatique qui sera utilisé pour la comptabilité du Groupe Harel Drouin. 
Le logiciel qui sera utilisé pour produire les feuilles de temps, les fichiers contenant les coordonnées de feuilles de temps et la liste des codes d'activités sera installé sur chaque micro-ordinateur. Chaque individu aura une disquette qui contiendra son identification, ses transactions de feuilles de temps et son fichier client. Le fichier du processus 2.1 contenant les transactions de feuilles de temps et certaines feuilles de temps sera employé par le processus "2.0 Compiler le temps" afin d'enregistrer les transactions facturables dans l'auxiliaire des travaux en cours dans le souscompte-client correspondant.

\subsubsection{Enregistrer données feuilles - temps}

Ce processus consiste à enregistrer l'emploi du temps à partir de l'agenda. Pour produire une feuille de temps, l'employé ou l'associé fournit à l'ordinateur la date de la feuille de temps ${ }^{(1)}$ pour laquelle il désire enregistrer de l'information. Le logiciel de feuille de temps valide cette date et lit, dans le fichier des coordonnées de feuilles de temps, les dates des jours qui doivent apparâtre sur la feuille de temps.

Par la suite, l'individu n'a qu'à inscrire la date de la journée pour laquelle il désire enregistrer une transaction, le code d'activité, le temps consacré à effectuer l'activité. Si l'activité est facturable, on enregistre également le numéro et le nom du client.

1- la date de la feuille de temps correspond à la date du samedi terminant la semaine. 


\subsubsection{Imprimer feuille de temps}

Pour imprimer sa feuille de temps, l'individu n'a qu'à fournir au logiciel la date de la feuille de temps. A l'aide de cette date, le logiciel lira, dans le fichier des coordonnées de feuilles de temps, les dates des jours compris dans la semaine de la feuille de temps demandée, le nombre d'heures régulières pour chaque journée ${ }^{(1)}$ et sélectionnera les transactions qui devront être imprimées.

Pour chaque journée comprise dans la feuille de temps, le logiciel imprimera les transactions (un numéro leur sera attribué lors de la saisie et ce numéro sera imprimé sur la feuille de temps), calculera le temps travaillé au cours de la journée et le temps supplémentaire (1e temps supplémentaire est calculé en fonction du temps facturable et du temps non facturable en omettant le temps consacré au cours de formation donnés ou suivis le samedi). Lorsque toutes les transactions auront été imprimées, le logiciel calculera le temps supplémentaire travaillé pour chaque journée et pour la semaine et imprimera le résultat de ses calculs.

\subsubsection{Corriger feuille de temps}

Pour corriger des transactions composant une feuille de temps, l'usager fournira au système le numéro de la transaction qu'il désire corriger. Le programme affichera la transaction à l'écran et l'individu n'aura qu'à modifier ce qu'il désire. Si une transaction est modifiée, le logiciel obligera l'individu à réimprimer sa feuille de temps.

1- le nombre d'heures régulières sert à calculer le temps supplémentaire. 


\subsubsection{Approuver feuilles de temps}

Toutes les feuilles de temps produites par les employés doivent être paraphées par le supérieur immédiat. Il arrive que le supérieur identifie une erreur. Dans ce cas, une correction est demandée et la feuille de temps est imprimée à nouveau.

\subsection{Saisir données feuilles de temps}

La saisie des données des feuilles de temps se compose de deux activités distinctes: la saisie des feuilles de temps produites manuellement et la saisie des feuilles de temps produites à l'aide de l'informatique. La saisie des feuilles de temps produites manuellement et la saisie des feuilles de temps produites à l'aide de l'informatique sont constituées d'activités différentes.

Par contre, dans les deux cas, le processus de saisie des feuilles de temps demande à l'usager de lui fournir la date pour laquelle il désire saisir des feuilles de temps. Le système vérifie si la date correspond à une date de feuille de temps qui a été préalablement enregistrée dans le système. Si aucune date n'y correspond, un message s'affichera. Dans le cas contraire, le système prend connaissance des dates des journées incluses dans la semaine de la feuille de temps. (1)

1- Ces dates seront utilisées pour sélectionner les transactions qui ont été saisies à l'aide de micro-ordinateur et pour valider les journées des transactions provenant de feuilles produites manuellement. 


\subsubsection{Saisie de feuilles de temps produites manuellement}

Lorsqu'un usager saisit des feuilles de temps produites manuellement, tous les champs saisis doivent être validés. Tous les champs d'une transaction devront être affichés à l'écran et l'usager peut modifier n'importe quel champ de la transaction en déplaçant son curseur par l'avant ou par l'arrière et ce, tant et aussi longtemps qu'il n'a pas mentionné au système qu'il doit accepter la transaction. Pour chaque feuille de temps à saisir, le système demandera le numéro de l'employé ou de l'associé pour lequel on désire enregistré des données de feuilles de temps et validera le numéro saisi avec ceux inscrits dans le fichier mâttre employés associés.

Pour chaque transaction à saisir, le système:

- validera la date de la transaction avec les dates des journées comprises dans la semaine de la feuille de temps

- demandera si l'activité est facturable.

Dans les cas d'activités facturables, le système:

- demandera le numéro et le nom du client

- s'assurera que les numéros et les noms fournis sont identiques à ceux inscrits dans le fichier maître des clients

- dans l'affirmative, le système demandera le code de l'activité, validera le code, affichera le nom de l'activité correspondant au code saisi

- dans le cas où il y a un client ayant le numéro saisi et dont le nom n'est pas le même, le système affichera le nom inscrit dans le fichier mâtre et demandera si c'est le bon client. Si ce n'est pas le bon client, le système supposera qu'il s'agit d'un nouveau client, effacera le numéro saisi et demandera à nouveau le nom du 
client (sans numéro de client, cette transaction sera inscrite dans le rapport de validation, voir section 2.2 )

- le système demandera le temps de l'activité et validera ce dernier (le temps doit être un multiple de 0.25 ).

- le système enregistrera la description du travail effectué.

Dans le cas d'activités non facturables:

- le système demandera le code de l'activité

- le système demandera le temps de l'activité et validera ce dernier (le temps doit être un multiple de 0.25).

- $\quad$ le système enregistrera la description du travail effectué.

2.8.2 Saisie des feuilles de temps produites à l'aide de l'informatique

Dans le cas de feuilles de temps produites à l'aide de l'informatique le processus est sensiblement le même.

Pour chaque transaction à saisir, le système:

- validera le numéro de l'employé ou associé avec ceux inscrits dans le fichier maître employés-associés.

- validera la date de la transaction avec les dates des journées comprises dans la semaine de la feuille de temps

- validera le code d'activité 
Dans le cas d'activités facturables, le système:

- s'assurera que le numéro et le nom fournis sont identiques à ceux inscrits dans le fichier maftre des clients

- le système lira le temps de l'activité et validera ce dernier (le temps doit être un multiple de 0.25).

- le système lira la description du travail effectué.

Dans le cas d'activités non facturables:

- le système lira le temps de l'activité et validera ce dernier (le temps doit être un multiple de 0.25).

- le système lira la description du travail effectué.

Dans un des cas suivants, le système mettra un code indiquant que la transaction n'est pas acceptée et cette dernière apparaftra automatiquement dans le rapport de validation:

- le numéro de l'employé ou de l'associé ne correspond à aucun numéro inscrit dans le fichier maître employés - associés.

- le code d'activité n'est pas valide

- le numéro et le nom du client ne correspond pas à ce qui est enregistré dans le fichier maftre-clients

- le temps de l'activité n'est pas un multiple de 0.25

- lorsqu'il n'y a pas de numéro de client 


\subsection{Valider les transactions feuilles - temps}

Ce processus est exécuté automatiquement par le système à chaque fois qu'une transaction est saisie ou corrigée. La validation des feuilles de temps consiste tout simplement à prendre chacune des transactions et à vérifier leur exactitude. Cette validation attribue à chaque transaction un code signifiant que la transaction est acceptée ou refusée et produit un rapport dans lequel on retrouve toutes les transactions dont le code de validation signifie que la transaction est refusée, c'est à dire celles:

- qui n'ont pas de numéro de client

- dont le nom du client ne correspond pas à celui qui est enregistré dans le fichier maitre-clients

- celles dont le code d'activité est égal à 0 (ces transactions ne sont pas considérées comme refusées)

- toutes les transactions dont le code de validation indique que la transaction n'est pas acceptée (s'applique aux transactions provenant de feuilles de temps produites à l'aide de l'informatique, référence section 2.8 .2 ).

\subsection{Corriger les transactions}

Le processus de correction d'une ou de plusieurs transactions nous permettra de corriger un ou plusieurs champs d'une transaction et de procéder à la saisie des noms des nouveaux clients. Pour ce faire, le système demandera à l'usager le type d'opération désiré (correction de transactions ou saisie de nouveaux clients). 


\subsubsection{Saisie de nouveaux clients}

Toutes les transactions, même celles qui n'apparaissent pas dans le rapport de validation, peuvent être corrigées par l'usager autorisé. Cependant, dès que le contenu d'une transaction est modifié, un code de non-validation est assigné à la transaction. Ce processus est suivi automatiquement du processus 2.2.

Dans le cas de saisie de noms de nouveaux clients, le système demande à l'usager de lui donner le nom du nouveau client. Il vérifie, dans le fichier mâtre-clients s'il y a un client ayant le même nom. Dans l'affirmative, le système en informe l'usager et lui demande s'il désire quand même créer ce nouveau client. Si l'usager répond par l'affirmative, un numéro de client sera désigné et l'usager n'aura plus qu'à sélectionner les transactions pour lesquelles il désire imputer le nom et le numéro de client. S'il n'y a pas de client ayant le même nom, le système désignera un numéro de client, l'usager n'aura qu'à sélectionner les transactions.

\subsubsection{Modification d'une transaction}

Dans le cas d'une modification, les transactions devront être triées dans l'ordre alphabétique des noms et prénoms d'employés ou associés afin que les transactions soient regroupées par feuilles de temps. Ainsi, il sera plus facile pour l'usager de retracer les transactions qu'il désire corriger. Tous les champs corrigés devront être validés comme pour la saisie des transactions de feuilles de temps. Dès que le contenu d'une transaction est changé, le code de validation est modifié de façon à ce qu'il signifie que la transaction n'a pas été validée. Tout comme la saisie des noms des nouveaux 
clients, la modification des transactions sera suivie automatiquement du processus 2.2 Valider les transactions.

2.4 Créer un nouveau client

Le processus de création d'un nouveau client est exécuté automatiquement à l'intérieur du processus de correction de transactions (processus 2.3). Cependant le système devra être assez souple pour permettre la création de nouveaux clients sans qu'il soit nécessaire de corriger des transactions. Ainsi, 1'usager pourra saisir les coordonnées d'un nouveau client avant même de procéder à la saisie de transactions. Dans ce cas, pour enregistrer le nom et le numéro du client dans les transactions, l'usager devra procéder par modification de transactions. (voir 2.3.1).

\subsection{Imputer le temps}

L'imputation du temps consiste à inscrire dans l'auxiliaire des travaux en cours toutes les transactions facturables pour un associé donné ou pour tous les associés. Le système demandera donc à l'usager le numéro de l'associé pour lequel il désire une imputation aux travaux en cours.

\subsubsection{Vérification de la validation}

Avant de procéder à l'imputation des travaux en cours, le système regardera dans le fichier qui contient toutes les transactions erronées (nommé Rapport de validation D2/1 dans le diagramme), s'il y a des transactions ayant le numéro de l'associé fourni 
par 1'usager. Dans l'affirmative, le système en informera l'usager et lui demandera s'il désire quand même procéder à la mise à jour.

\subsubsection{Vérification de la réception de toutes les feuilles de temps}

Avant de procéder à l'imputation des travaux en cours, le système devra également vérifier si toutes les feuilles de temps de l'associé ont été enregistrées dans le système. Pour procéder à cette vérification, le système regardera dans un fichier nommé D2/5 Feuilles de temps manquantes s'il y a des enregistrements ayant le numéro de l'associé fourni par l'usager. Dans l'affirmative, le système en informera l'usager et lui demandera s'il désire quand même procéder à l'imputation des transactions.

\subsubsection{L'imputation des transactions}

Finalement, pour imputer les transactions dans les travaux en cours, le système lira chacune des transactions contenues dans le fichier des transactions de feuilles de temps D2/6. Pour chaque transaction lue, il regardera si la transaction contient le numéro de l'associé fourni. Si la transaction est validée, si elle contient le numéro fourni et que son code d'activité signifie que c'est une transaction non facturable, cette dernière sera inscrite dans le fichier "D10 Cumulatif des transactions". Si le code de la transaction signifie que son temps est facturable, le système lira dans le fichier maitre employés-associés le taux horaire correspondant au numéro de l'employé ou associé concerné par la transaction, multipliera ce taux horaire par le temps de la transaction et inscrira la transaction et son montant dans l'auxiliaire des travaux en cours. 
Le système additionnera le montant de la transaction au cumulatif de l'associé afin de pouvoir générer l'écriture de journal reliée à la mise à jour des travaux en cours. Cette écriture sera enregistrée dans le fichier "D8 Écritures de journal".

Le code d'imputation de la transaction lue dans le fichier "D2/6 transactions feuilles de temps" (que la transaction soit facturable ou non facturable) sera modifié afin qu'il signifie que la transaction a été traitée).

Le système inscrira, dans le fichier "D2/7 Dernière date d'imputation", le numéro de l'associé et la date de l'imputation de ces travaux en cours.

\subsection{Produire un rapport statistique}

Ce processus consiste à produire différents rapports statistiques demandés par les usagers. Pour connaitre les rapports statistiques produits par ce processus, consultez le dictionnaire section "2.6 Produire rapports statistiques."

\subsection{Contrôler feuilles de temps}

Ce processus est exécuté automatiquement par le système de façon continuelle. Il est donc transparent à l'utilisateur. Son objectif est de vérifier continuellement l'enregistrement des feuilles de temps dans le système et de permettre de connaître les

noms des personnes qui n'ont pas remis leur feuille de temps et ce, pour un associé donné. Cette vérification est effectuée pour toutes les semaines de feuilles de temps non complétées excluant la semaine courante. 


\subsubsection{Déterminer les feuilles manquantes}

Ce processus consiste à vérifier s'il y a, pour chaque semaine de feuilles de temps non-complétée et pour chaque employé ou associé actif inscrit dans le fichier maître employés-associés, une transaction ayant le numéro lu dans le fichier maître mentionné et la date d'une des journées lues dans le fichier des coordonnées de feuilles de temps.

A chaque fois que le processus de contrôle des feuilles de temps est exécuté, le contenu du fichier des feuilles de temps manquantes est modifié. Ainsi, lorsqu'aucune inscription pour une semaine donnée n'est pas enregistrée dans le fichier des feuilles de temps manquantes $\mathrm{D} 2 / 5$, un code est enregistré dans le fichier des coordonnées de feuilles de temps pour indiquer que toutes les feuilles de temps ont été reçues.

\subsubsection{Vérifier les feuilles manquantes}

Ce processus consiste à afficher à l'écran ou à imprimer dans un rapport, la liste des personnes n'ayant pas remis leur feuille de temps, et ce, pour une semaine et un associé.

L'employé autorisé fournit au système la date de la feuille de temps pour laquelle il désire procéder à la vérification et le numéro de l'associé pour lequel la vérification est désirée. Si aucun numéro d'associé n'est fourni au système, celui-ci affichera la liste pour tous les associês. 


\subsubsection{Calculer le temps supplémentaire}

Lorsque toutes les feuilles de temps ont été reçues, le système procède au calcul du temps supplémentaire. Pour cette opération, le système doit trier toutes les transactions dans l'ordre de date et dans l'ordre de numéro d'employés ou d'associés. Pour chaque journée de la semaine de feuilles de temps qui vient d'être identifiée comme étant complétée, pour chaque employé ou associé, le système additionnera le temps de toutes les transactions de la journée (en omettant les transactions dont le code d'activité signifie " cours de formation donnés ou suivis le samedi"). Ce temps sera soustrait au temps régulier de la journée qui est inscrit dans le fichier des coordonnées de feuilles de temps. Si le résultat de la soustraction est différent de zéro, la date de la transaction, le numéro de l'employé ou de l'associé, sera inscrit dans le fichier "D10 Cumulatif des transactions" avec un code d'activité signifiant que la transaction représente du temps supplémentaire.

Une fois que les transactions de temps supplémentaire ont été inscrites dans le fichier "D10 Cumulatif des transactions", le système inscrira, dans le fichier "D10 Cumulatif des transactions", toutes les transactions facturables de feuilles de temps dont le code d'imputation signifie qu'elles ont été utilisées pour l'imputation des travaux en cours et les effacera du fichier "D2/6 transactions de feuilles de temps". 
5. Effectuer la comptabilité

\subsection{Facturer les clients}

Dans ce processus, vous allez constater que les factures seront dictées et dactylographiées, et que les numéros de référence des factures assignés par le système informatique seront transcrits sur les factures. Le client désirait qu'il est en soit ainsi. Nous sommes conscients que ce n'est pas la façon la plus efficace de procéder et nous croyons que le client le réalisera tôt ou tard. Pour cette raison, nous désirons que le système offre la possibilité de générer des factures directement à partir de l'auxiliaire des travaux en cours. Le texte de ces factures sera conçu à partir de paragraphes préalablement enregistrés dans le système informatique.

\subsubsection{Produire la facture}

Pour produire une facture, l'associé ou le chef d'équipe consulte l'auxiliaire des travaux en cours et dicte la facture à sa secrétaire.

\subsubsection{Dactylographier la facture}

A partir de l'information dictée, la secrétaire rédige la facture sur son appareil de traitement. Ensuite, sur du papier vert, elle effectue une copie. Cette copie est remise au département de la comptabilité. 


\subsubsection{Saisir la facture}

La première étape avant d'enregistrer la facture dans le système informatique est d'additionner les montants des factures. La somme obtenue sera utilisée pour vérifier l'exactitude de la saisie en comparant la somme calculée par l'ordinateur avec la somme obtenue à partir des factures. Le système devra nous permettre d'imprimer les factures saisies afin de faciliter la recherche d'erreurs.

Lors de la saisie, le système informatique assignera un numéro de référence à la facture. Ce numéro de référence sera inscrit sur la copie verte de la facture. II apparaitra dans le journal de la facturation.

\subsubsection{Enregistrer la vente}

L'enregistrement de la vente consiste à inscrire les factures saisies dans les fichiers des auxiliaires des comptes à recevoir et des travaux en cours.

Lors de l'enregistrement de la vente, le système additionnera les montants de chaque facture. La somme obtenue sera employée pour générer l'écriture de journal qui aura comme conséquence de modifier, dans le grand-livre, les soldes des comptes à recevoir et des travaux en cours. La description associée à l'écriture sera générée automatiquement et sera suivie de la date enregistrée dans le système informatique. 


\subsubsection{Saisir les chèques}

La saisie des chèques envoyés par les clients aura pour conséquence de modifier les soldes des comptes à recevoir et de la banque. Lorsque nous saisirons les chèques, nous enregistrons le numéro du client, la date de son chèque, le numéro de référence de la facture visée par le chèque et le montant du chèque. Le système devra nous permettre de consulter l'auxiliaire des comptes à recevoir afin d'identifier le numéro de référence de la facture, et ce, sans quitter la présente opération.

\subsubsection{Enregistrer les recettes}

L'enregistrement des recettes consiste à inscrire les chèques saisis dans l'auxiliaire des comptes à recevoir.

Lors de l'enregistrement des recettes, tous les montants des chèques sont additionnés ensemble. Le montant obtenu sera utilisé pour générer l'écriture de journal qui aura comme conséquence de modifier, dans le grand-livre, les soldes des comptes à recevoir et de la banque. La description associêe à l'écriture sera générée automatiquement et sera suivie de la date enregistrée dans le système informatique.

\subsection{Payer les fournisseurs}

Dans le processus "Payer les fournisseurs", nous avons automatisé le maximum de tâches. Ce processus contient des opérations qui affectent automatiquement les registres comptables d'une corporation et d'une société et ce, sans intervention humaine. 


\subsubsection{Autoriser les factures}

L'autorisation des factures consiste seulement à parapher les factures ou les comptes de dépenses par un associé ou un employé autorisé (1). Lorsqu'une facture est paraphée, cela signifie que le paiement en est autorisé. Les factures et comptes de dépenses autorisés sont acheminés au dêpartement de la comptabilitê afin d'inscrire les codes d'imputation.

\subsubsection{Inscrire les codes d'imputation}

Ce processus consiste, pour chaque facture ou compte de dépenses autorisé, à inscrire sur la facture ou le compte de dépenses, les numéros de comptes de grandlivre dans lesquels seront assignés les différents éléments de la facture et le numéro du compte fournisseur. Dans le cas de dépenses facturables, le numéro du client est inscrit sur la facture.

\subsubsection{Saisir les factures}

La saisie des factures consiste à enregistrer dans le système, les codes d'imputation et le montant attribué à chacun d'eux. Lors de cette saisie, le système affichera le nom du fournisseur correspondant au numéro saisi (les employés présentant des comptes de dépenses sont considérés comme fournisseurs) et affichera la description du compte de grand-livre correspondant au numéro saisi. Ce processus génèrera automatiquement les écritures de journal et reportera automatiquement lesdites écritures dans

1- Seul l'employé responsable de l'informatique ou de la comptabilité peut autoriser le paiement de factures. 
le grand-livre. Pour certains comptes de dépenses, le système demandera le numéro du client. Ce numéro sera employé lors du processus 5.5.7 Imputer les dépenses pour imputer les dépenses facturables dans l'auxiliaire des travaux en cours.

\subsubsection{Imprimer la liste des factures à payer}

Pour imprimer la liste des factures à payer, le système regardera dans le fichier maitre-fournisseurs le nombre de jours après lesquels les factures de chaque fournisseur deviennent à échéance. Avec cette information, pour chaque facture inscrite dans l'auxiliaire des comptes à payer, le système calculera la date d'échéance. Si la date d'échéance de la facture est inférieure ou égale à la date fournie par 1'usager, le système imprimera la facture dans la liste des factures à payer. Sera également inscrites dans la liste des comptes à payer, les coordonnées des fournisseurs qui ont une ou plusieurs factures à payer.

\subsubsection{Retenir certaines factures}

Cette opération consiste à sélectionner les factures qui ne seront pas payées ou celles pour lesquelles un paiement partiel sera effectué. Un code indiquant que 1a facture est retenue ou qu'un paiement partiel sera effectué sera inscrit dans l'auxiliaire des comptes à payer. Dans les cas de paiements partiels, l'information relative aux paiements sera inscrite dans un fichier destiné à cet effet.

Durant cette opération, le personnel retirera des dossiers contenants les factures à payer toutes les factures qui seront payées. Ces factures seront classées avec les copies de chèques dans les dossiers des fournisseurs. 


\subsubsection{Imprimer les chèques}

Les chèques sont imprimés par le système informatique dès que la liste des factures à payer ne nécessite plus de corrections. Pour chaque fournisseur inscrit dans la liste finale des factures à payer, un chèque couvrant l'ensemble des factures payables (1) sera émis. Les coordonnées du fournisseur nécessaires à la production du chèque proviennent du fichier mastre-fournisseurs.

Le numéro, la date et le montant de chaque facture seront imprimés sur le talon du chèque. Le montant du chèque sera calculé à partir des montants des factures imprimés sur le talon du chèque. Les copies des chèques seront classées avec les factures payées et le tout sera rangé dans les dossiers des fournisseurs.

L'impression des chèques produira des écritures automatiques qui affecteront les comptes à payer, l'auxiliaire des comptes à payer et le compte de banque correspondant.

\subsubsection{Imputer les dépenses}

Ce processus visent à reporter automatiquement toutes les dépenses encourues par la corporation "Les Services Harel Drouin" dans les livres de la société "Harel Drouin et associés". Des écritures automatiques seront générées et auront comme conséquence de créditer les comptes de dépenses de la corporation, de débiter le compte INTER-compagnie

1- c'est à dire les factures pour lesquelles il n'y a pas de code de retenu. Dans les cas de factures pour lesquelles une code indiquant qu'un paiement partiel sera effectué, le système regardera dans le fichier des paiement partiels, le montant du paiement. 
de la corporation, de débiter les comptes de dépenses de la société (pour les dépenses non facturables), de débiter le comptes de travaux en cours (pour les comptes de dépenses facturables) et de créditer le compte intercompagnie de la société. L'imputation des dépenses pourra générer les écritures suivantes:

\section{Dans le cas de la corporation:}

INTER $\quad \mathrm{Xxx}$

$$
\text { dépenses } \mathrm{xxx}
$$

Dans le cas de la société:

$\begin{array}{lll}\text { dépenses } & \mathrm{xxx} \\ \text { travaux en cours } & \mathrm{xxx} & \\ \text { INTER } & & \mathrm{xxx}\end{array}$

Nous incluons également dans ce processus, 1'inscription, dans 1e fichier "D10 Cumulatif des transactions", des dépenses non facturables provenant des comptes de dépenses des employés. Cela nous permettra de contrôler ces dépenses via la production des rapports statistiques (voir processus 2.0 Imputer le temps).

\subsubsection{Enregistrer les nouveaux fournisseurs}

Il s'agit d'enregistrer le nom du fournisseur, son adresse et son délai de paiement. 


\subsection{Produire les rapports financiers}

Nous désirons informer le lecteur que ce processus n'a pas été validé auprès du directeur administratif. Ce dernier a quitté ses fonctions récemment et le poste demeure vacant.

Dans cette section, le terme "période" réfère à un mois de l'année financière. Le système permettra d'enregistrer des transactions même si une nouvelle année financière est commencée.

Le processus a comme objectif d'informer les sociétaires ou les actionnaires sur la situation financière de leur entreprise. Il peut être exécuté mensuellement ou annuellement. Dans le cas où il est exécuté mensuellement, l'activité 5.6.2 Saisir les régularisations ne sera pas effectuée.

Tous les rapports demandés par l'usager peuvent être affichés à l'écran ou imprimés. Le système permet également de consulter à l'écran, les transactions et le solde d'un compte du grand-livre.

\subsubsection{Saisir les régularisations}

Ce processus, exécuté après la fin de l'année financière, permet d'enregistrer des écritures qui modifieront les soldes de certains comptes du grand-1ivre. A titre d'exemple, une erreur au niveau d'un numéro de compte de grand-livre peut justifier une écriture de régularisation. Les écritures de régularisations peuvent être enregistrées dans n'importe quelle période financière. 


\subsubsection{Enregistrer les écritures automatiques}

Dans tous les systèmes comptables, il y a des écritures dont le montant demeure constant et qui affectent mensuellement les mêmes comptes du grand-livre. Pour éviter de répéter mensuellement la saisie de ces écritures, le système nous permet d'enregistrer ces écritures qu'une seule fois au début de l'année financière. Automatiquement, le système procédera à l'inscription de ces écritures dans les registres comptables.

\subsubsection{Produire le grand-livre}

La production du grand-livre génère un document dans lequel on retrouve, pour chaque compte du grand-livre:

- le solde du début de la période

- les transactions de la période

- le solde à la fin de la période.

Pour produire le grand-livre, le système informatique trie les transactions par ordre de numéro de compte et de date. Pour calculer le solde au début de la période, il compare le mois de la transaction avec la période. Dans les cas où le mois est inférieur à la période, le montant de la transaction est additionné. Lorsqu'il n'y a plus de transaction dont le mois est inférieur à la période, le système imprimera le solde calculé. Dans le cas où le mois de la transaction est égal à la përiode, la transaction est imprimée, et son montant additionné. Dans le cas où le mois de la transaction est supérieur à la période, la transaction n'est pas imprimée et son montant est ignoré. 
Lorsque le système a traité toutes les transactions ayant le même numéro de compte, il imprime le solde du compte à la fin de la période.

\subsubsection{Produire le grand-livre cumulatif}

La production du grand-livre cumulatif génère un document dans lequel on retrouve toutes les transactions comptables ayant modifié la situation financière des entreprises.

Pour produire le grand-livre cumulatif, le système informatique trie les transactions dans l'ordre de numéro de compte et de date. Les transactions sont imprimées dans l'ordre utilisé pour le tri. Lorsque le système imprime les transactions d'un compte, il additionne le montant des transactions. Lorsque toutes les transactions d'un compte sont imprimées, le système imprime le solde obtenu. Le même processus est exécuté pour tous les comptes d'opération et de bilan.

\subsubsection{Produire l'auxiliaire des travaux en cours.}

L'impression de l'auxiliaire des travaux en cours génère un rapport dans lequel on retrouve l'ensemble des transactions ayant modifié les comptes clients. Le système nous permet une impression sélective des comptes-clients. Nous pouvons imprimer l'auxiliaire des travaux en cours pour l'ensemble des clients, pour certains clients ou pour un seul. 
L'auxiliaire des travaux en cours est imprimé pour une période donnée et il nous fournit de l'information sur les soldes (1) au début de la période, les transactions de la pêriode et les soldes de la fin de période.

\subsubsection{Produire 1'auxiliaire des comptes à recevoir}

L'impression de l'auxiliaire des comptes à recevoir génère un rapport dans lequel on retrouve l'ensemble des transactions ayant modifié les comptes-clients et ce, peu importe la date des transactions. Le système nous permet une impression sélective des comptes à recevoir. Nous pouvons imprimer l'auxiliaire pour l'ensemble des clients, pour certains clients ou pour un seul. Le système nous permet également d'imprimer toutes les factures inscrites dans 1'auxiliaire ou seulement les factures pour lesquelles nous avons reçu un paiement partiel ou pour lesquelles aucun paiement n'a été reçu.

\subsubsection{Produire l'auxiliaire des comptes à payer}

L'impression de l'auxiliaire des comptes à payer génère un rapport dans lequel on retrouve l'ensemble des transactions ayant modifié les comptes des fournisseurs et ce, peu importe la date des transactions. Le système nous permet une impression sélective des comptes à payer. Nous pouvons imprimer l'auxiliaire pour l'ensemble des fournisseurs, pour certains ou pour un seul. Le système nous permet également d'imprimer toutes les factures inscrites dans l'auxiliaire ou seulement les factures pour lesquelles nous avons effectué un paiement partiel ou pour lesquelles aucun paiement n'a été effectué.

1- solde de profit et perte, de facturation, de temps et de déboursés 
5.6.8 Produire les états financiers

La production des états financiers vise à compiler l'ensemble des opérations de l'entreprise dans le but de produire un document représentant l'image de l'entreprise à une période précise dans l'année. Dans le cas des entreprises du Groupe Hare1, Drouin, la fin de l'année fiscale est le 31 janvier.

Pour produire les états financiers, on additionne au solde du début l'ensemble des transactions ayant affecté chaque compte du grand-livre. On regroupe également ensemble plusieurs soldes afin d'obtenir des regroupements de postes. 
Annexe I

\section{Dictionnaire}

Ancien système 


\section{TABLE DES MATIÈRES}

\section{Processus 2.0, Compiler le temps …....... 1}

Diagrammes de flux de données $\ldots \ldots \ldots \ldots \ldots \ldots \ldots \ldots$

2.0 Compiler le temps $\ldots \ldots \ldots \ldots \ldots \ldots \ldots \ldots \ldots \ldots \ldots \ldots \ldots \ldots$

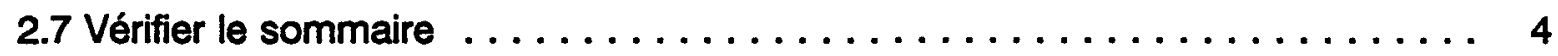

Processus de traitement $\ldots \ldots \ldots \ldots \ldots \ldots \ldots \ldots \ldots \ldots \ldots$ 5

Enregistrer les données de base, $2.1 \ldots \ldots \ldots \ldots \ldots \ldots \ldots \ldots \ldots \ldots 6$

Enregistrer les données pour la feuille de temps, $2.2 \ldots \ldots \ldots \ldots \ldots \ldots .7$

Imprimer la feuille de temps, $2.3 \ldots \ldots \ldots \ldots \ldots \ldots \ldots \ldots \ldots \ldots .6$

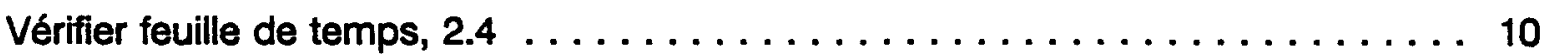

Procéder à la fermeture de la semaine, $2.5 \ldots \ldots \ldots \ldots \ldots \ldots \ldots \ldots \ldots 11$

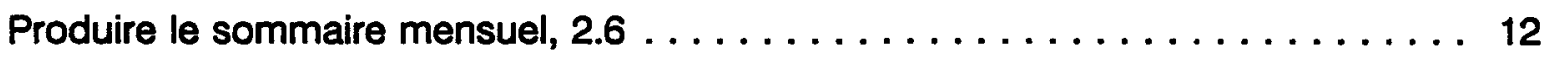

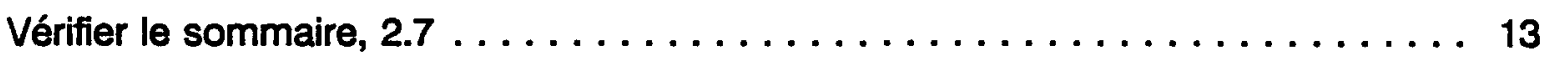

Enregistres les numéros manquants $2.7 .1 . \ldots \ldots \ldots \ldots \ldots \ldots \ldots \ldots \ldots$

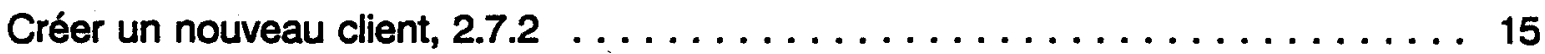

Vérifier la saisie des sommaires, $2.8 \ldots \ldots \ldots \ldots \ldots \ldots \ldots \ldots \ldots \ldots \ldots$

Procéder à la fermeture du mois, $2.9 \ldots \ldots \ldots \ldots \ldots \ldots \ldots \ldots \ldots \ldots$

Approuver la feuille de temps, $2.10 \ldots \ldots \ldots \ldots \ldots \ldots \ldots \ldots \ldots \ldots \ldots 18$

Flux de données $\ldots \ldots \ldots \ldots \ldots \ldots \ldots \ldots \ldots \ldots \ldots \ldots$

Nom et temps du dossier, b-2.2 $\ldots \ldots \ldots \ldots \ldots \ldots \ldots \ldots \ldots \ldots \ldots \ldots 20$

Données de base, b-2.1 ............................. 21

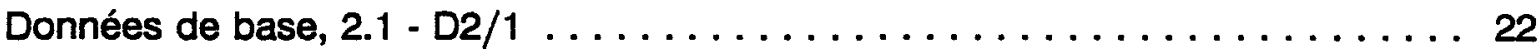

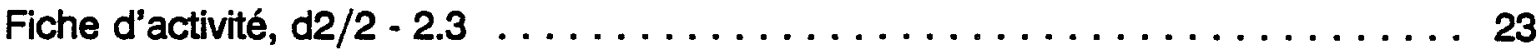

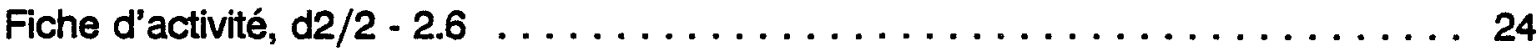




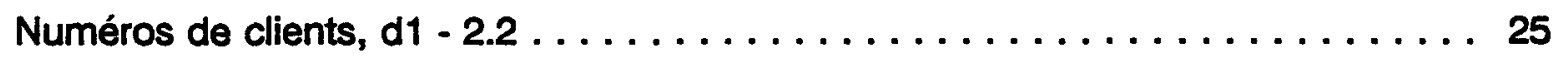

Numéros de clients, $d t-2.7 .1 \ldots \ldots \ldots \ldots \ldots$

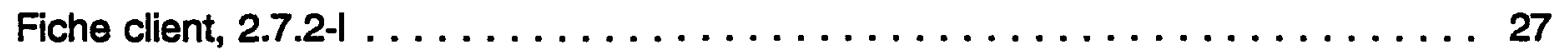

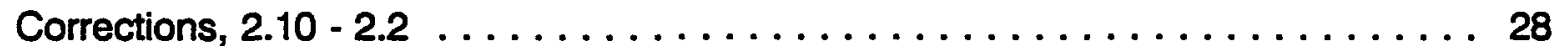

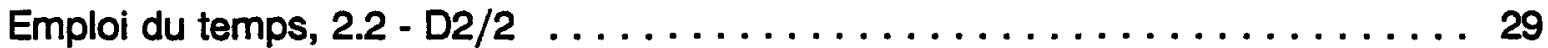

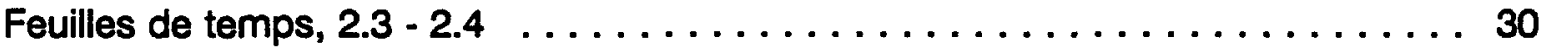

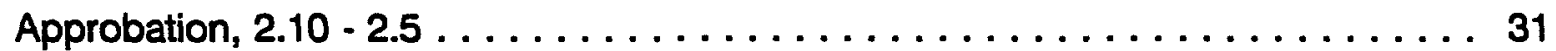

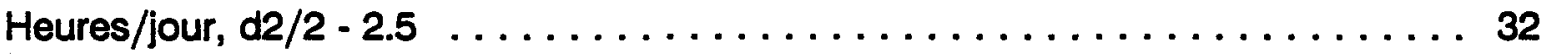

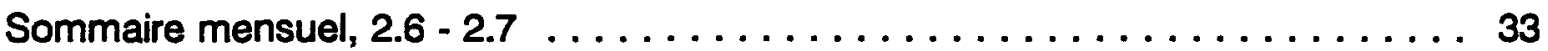

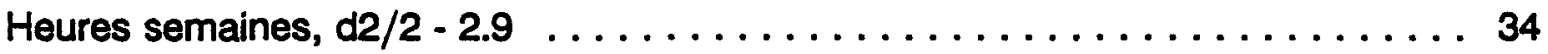

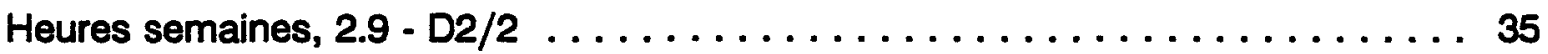

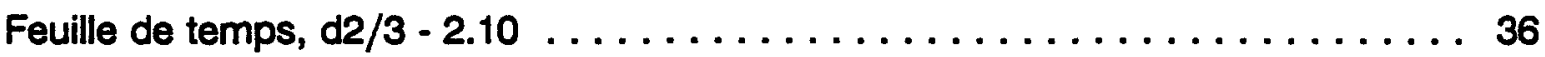

Heures facturables et non facturables $2.9-\mathrm{D} 2 / 5 \ldots \ldots \ldots \ldots$

Validation des feuilles de temps, e-2.8 . . . . . . . . . . . . . . . 38

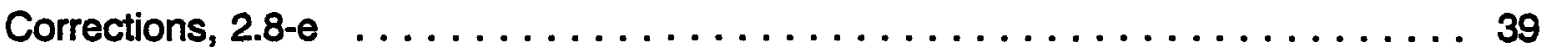

\section{Processus 5.1, Enregistrer les ventes ...... 40}

Diagrammes de flux de données $\ldots \ldots \ldots \ldots \ldots \ldots \ldots . \ldots 41$

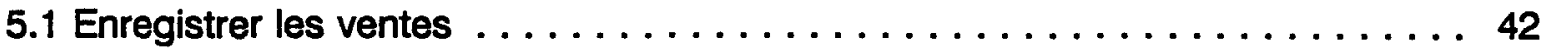

Processus de traitement $\ldots \ldots \ldots \ldots \ldots \ldots \ldots \ldots \ldots \ldots 43$

Préparer la facture, $5.1 .1 \ldots \ldots \ldots \ldots \ldots \ldots$

Dactylographier la facture, $5.1 .2 \ldots \ldots \ldots \ldots \ldots \ldots$

Reproduire les factures, $5.1 .3 \ldots \ldots \ldots \ldots \ldots \ldots$

Vérifier les copies de factures, $5.1 .4 \ldots \ldots \ldots \ldots \ldots \ldots$

Numéroter les copies de factures, $5.1 .5 \ldots \ldots \ldots \ldots$. . . . . . . . . 48

Additionner le copies de factures, $5.1 .6 \ldots \ldots \ldots \ldots \ldots$

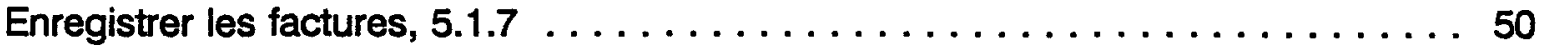

Vérifier l'enregistrement des factures, $5.1 .8 \ldots \ldots \ldots \ldots \ldots \ldots \ldots \ldots \ldots \ldots$ 


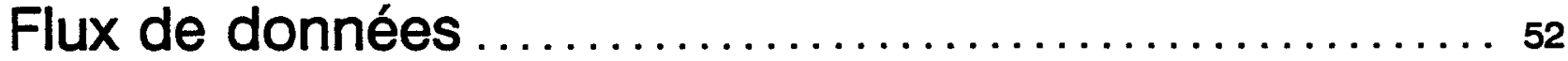

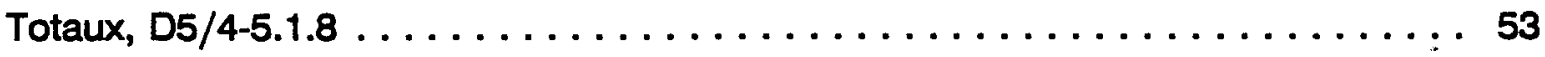

Total copies vertes acceptées $\ldots \ldots \ldots \ldots \ldots \ldots \ldots \ldots \ldots \ldots \ldots \ldots 4$

Copies vertes factures, $5.1 .7-D 5 / 2 \ldots \ldots \ldots \ldots \ldots \ldots \ldots \ldots \ldots \ldots \ldots$

Copies vertes factures, $5.1 .7-D 5.1 / 4 \ldots \ldots \ldots \ldots \ldots \ldots \ldots \ldots \ldots \ldots \ldots 6$

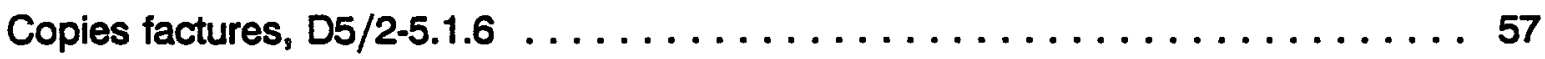

Formulaires de transactions, $D 5 / 8-e \ldots \ldots \ldots \ldots \ldots \ldots \ldots \ldots \ldots \ldots \ldots$

Formulaires de transaction, $5.1 .6-\mathrm{D} 5 / 8 \ldots \ldots \ldots \ldots \ldots \ldots \ldots \ldots \ldots \ldots$

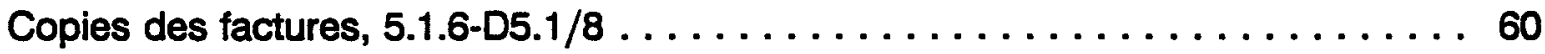

Copies des factures, $D 5.1 / 8-e \ldots \ldots \ldots \ldots \ldots \ldots \ldots \ldots \ldots \ldots \ldots 61$

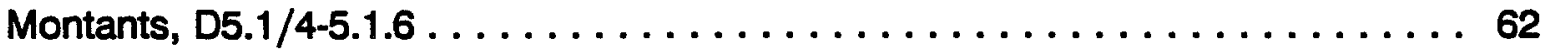

Copies de factures, $D 5.1 / 3-5.1 .6 \ldots \ldots \ldots \ldots \ldots \ldots \ldots \ldots \ldots \ldots 6 . \ldots \ldots \ldots \ldots$

Copies vertes des factures $\mathrm{D} 5.1 / 3-5.1 .7 \ldots \ldots \ldots \ldots \ldots \ldots \ldots \ldots \ldots \ldots 64$

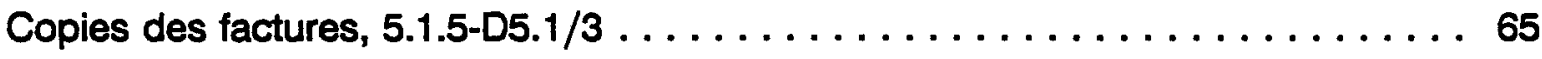

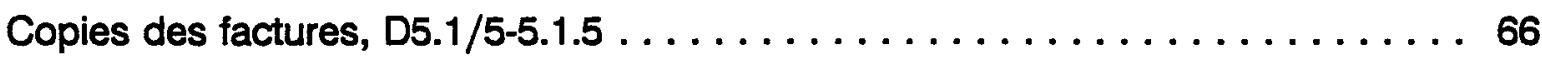

Copies des factures, $5.1 .4-D 5.1 / 5 \ldots \ldots \ldots \ldots \ldots \ldots \ldots \ldots \ldots \ldots 67$

Copies des factures, $05.1 / 1-5.1 .4 \ldots \ldots \ldots \ldots \ldots \ldots \ldots \ldots \ldots \ldots \ldots 68$

Copie blanche, $5.1 .3-D 5 / 7 \ldots \ldots \ldots \ldots \ldots \ldots \ldots \ldots \ldots \ldots \ldots 69$

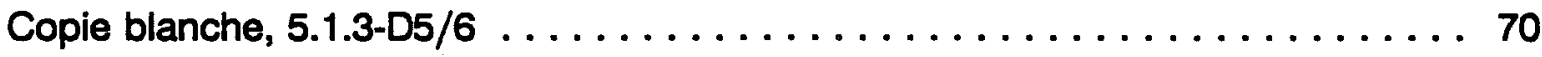

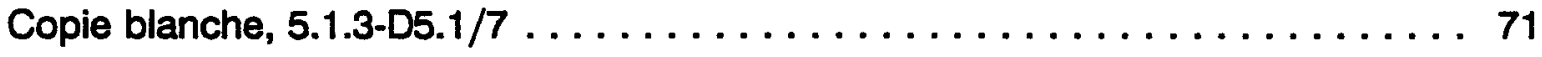

Copies de couleur, $5.1 .3-05.1 / 1 \ldots \ldots \ldots \ldots \ldots \ldots \ldots \ldots \ldots \ldots \ldots 72$

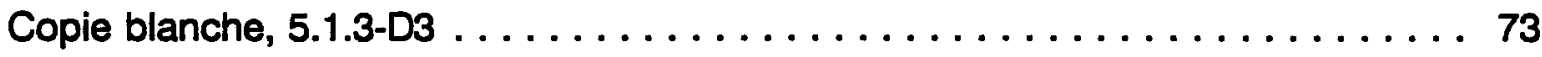

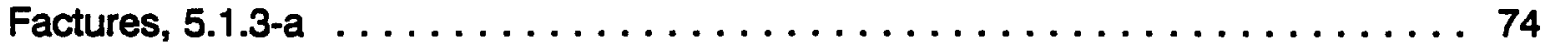

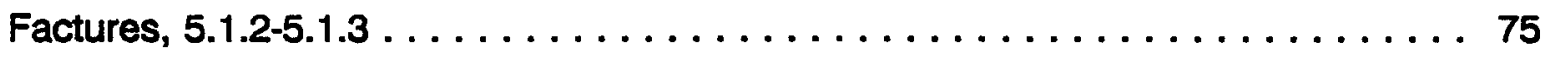

Brouillon de factures, $5.1 .1-5.1 .2 \ldots \ldots \ldots \ldots \ldots \ldots \ldots \ldots \ldots \ldots \ldots$

Travail effectué, b-5.1.1 $\ldots \ldots \ldots \ldots \ldots \ldots \ldots \ldots \ldots \ldots \ldots \ldots \ldots$

Nombre d'heures, b-5.1.1 ........................ 78

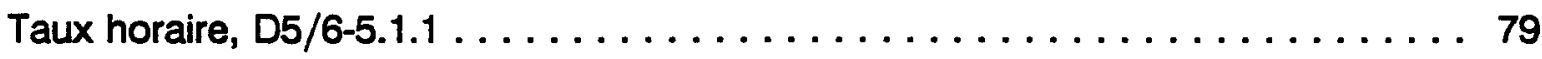

Montant imputé, $05 / 6-5.1 .1 \ldots \ldots \ldots \ldots \ldots \ldots \ldots \ldots \ldots \ldots \ldots . \ldots \ldots$

Copie facture antérieure, $\mathrm{d} 3-5.1 .1 \ldots \ldots \ldots \ldots \ldots \ldots \ldots \ldots \ldots \ldots . \ldots 1$ 


\section{Processus 5.2, Enregistrer les recettes .... 82}

Diagrammes de flux de données $\ldots \ldots \ldots \ldots \ldots \ldots \ldots$.........

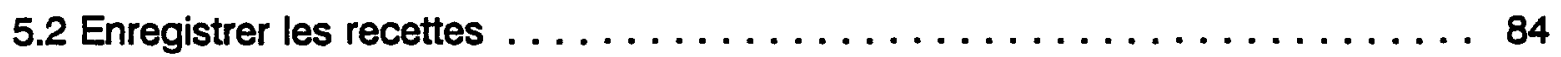

Processus de traitement $\ldots \ldots \ldots \ldots \ldots \ldots \ldots \ldots \ldots \ldots . \ldots . \ldots . \ldots$

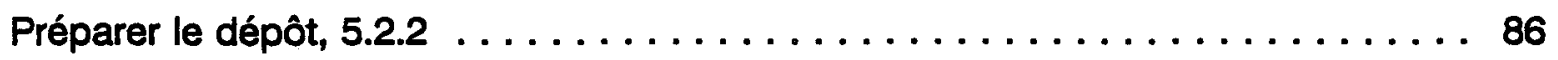

Enregistrer les chèques, $5.2 .1 \ldots \ldots \ldots \ldots \ldots \ldots \ldots \ldots \ldots \ldots$

Additionner les montants, $5.2 .3 \ldots \ldots \ldots \ldots \ldots \ldots \ldots \ldots$

Comparer les soldes, $5.2 .3 \ldots \ldots \ldots \ldots \ldots \ldots$. . . . . . . . . . . 89

Enregistrer le numéro de la facture, $5.2 .5 \ldots \ldots \ldots \ldots \ldots \ldots$

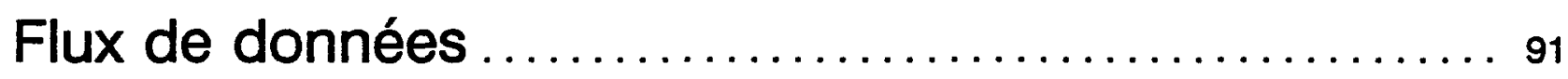

Numéro du client, d1-5.2.1 . . . . . . . . . . . . . . . . . 92

Information relative au chèque, $5.2 .1-D 5 / 8 \ldots \ldots \ldots \ldots \ldots$

Chèques, $5.2 .1-5.2 .2 \ldots \ldots \ldots \ldots \ldots \ldots \ldots \ldots \ldots \ldots \ldots \ldots$

Nom du client, $f-5.2 .1 \ldots \ldots \ldots \ldots \ldots \ldots \ldots$

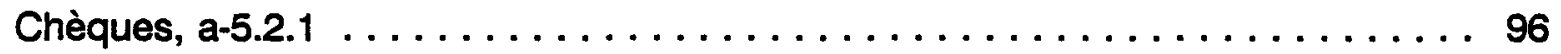

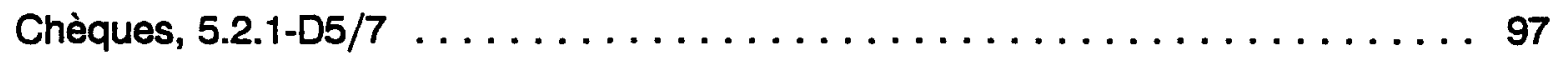

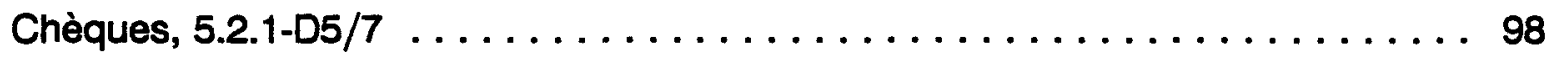

Montant du dépôt, $5.2 .2-5.2 .4 \ldots \ldots \ldots \ldots \ldots \ldots \ldots \ldots$

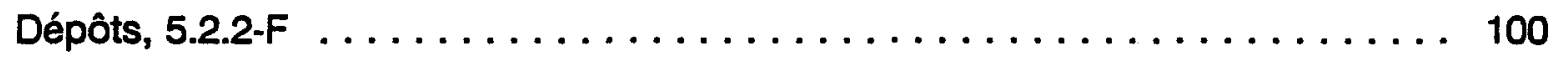

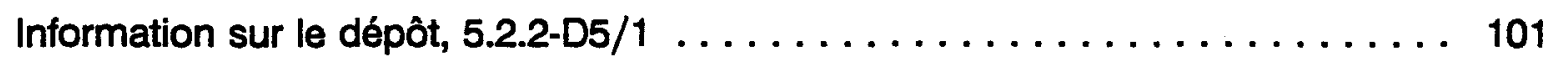

Numéro de facture, $5.2 .5-D 5 / 8 \ldots \ldots \ldots \ldots \ldots \ldots \ldots$. . . . . . . . . . . 102

Numéro de facture, $05 / 2-5.2 .5 \ldots \ldots \ldots \ldots \ldots \ldots \ldots \ldots \ldots$

Numéro de facture, $\mathrm{D} 5 / 5-5.2 .5 \ldots \ldots \ldots \ldots \ldots \ldots \ldots$. . . . . . . . . . . . 104

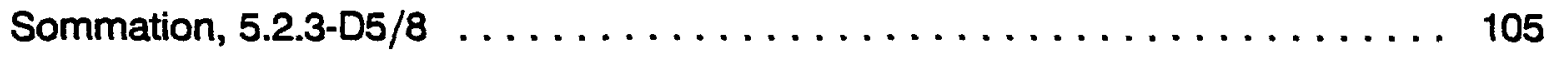

Formulaire de transactions, $D 5 / 8-e \ldots \ldots \ldots \ldots \ldots \ldots \ldots \ldots$

Sommation des transactions, $5.2 .3 \ldots \ldots \ldots \ldots \ldots \ldots \ldots \ldots \ldots$

Montant, $D 5 / 8-5.2 .3 \ldots \ldots \ldots \ldots \ldots \ldots \ldots \ldots \ldots \ldots$ 


\section{Processus 5.4, Concilier les comptes de banque

Diagrammes de flux de données $\ldots \ldots \ldots \ldots \ldots \ldots \ldots \ldots, 110$

5.4 Concilier les comptes de banque $\ldots \ldots \ldots \ldots \ldots \ldots \ldots \ldots \ldots \ldots$

Processus de traitement $\ldots \ldots \ldots \ldots \ldots \ldots \ldots \ldots \ldots \ldots, 112$

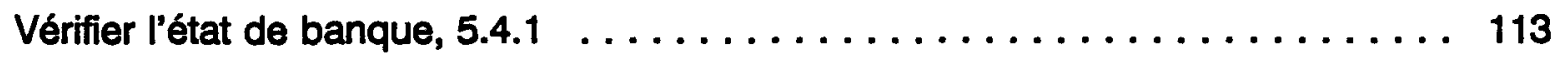

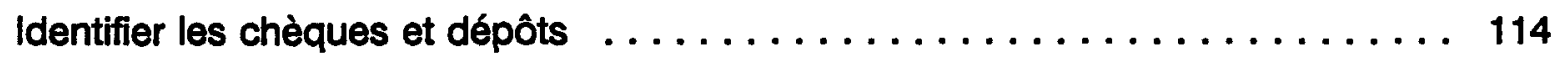

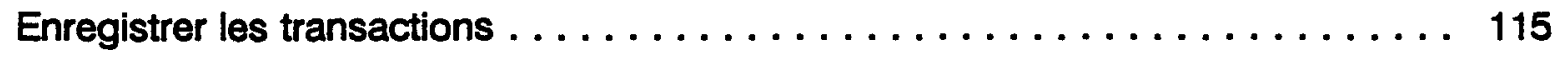

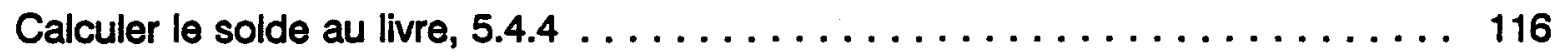

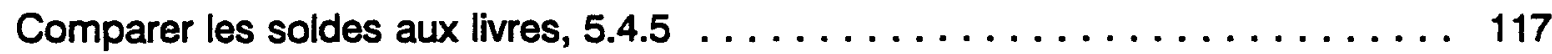

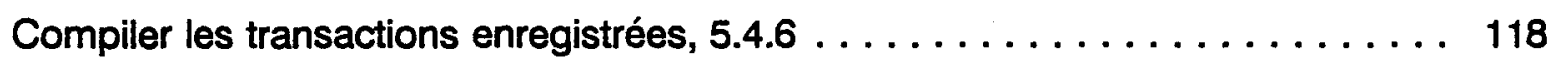

Flux de données ................................ 119

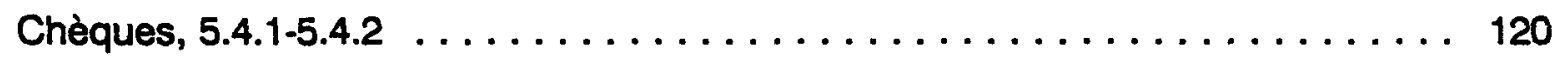

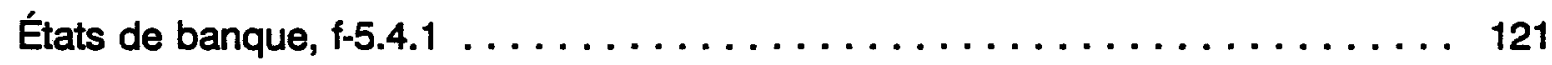

Chèques, f-5.4.1 $\ldots \ldots \ldots \ldots \ldots \ldots \ldots \ldots \ldots \ldots \ldots \ldots \ldots \ldots \ldots, 122$

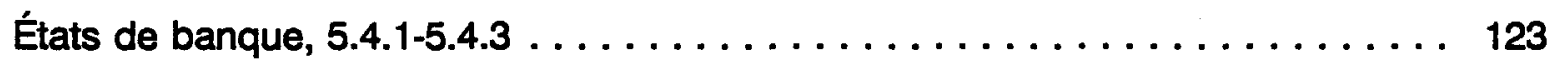

États de banque, $5.4 .3,05.4 / 4 \ldots \ldots \ldots \ldots \ldots \ldots \ldots \ldots \ldots \ldots \ldots \ldots 124$

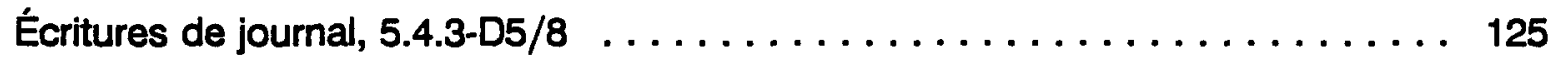

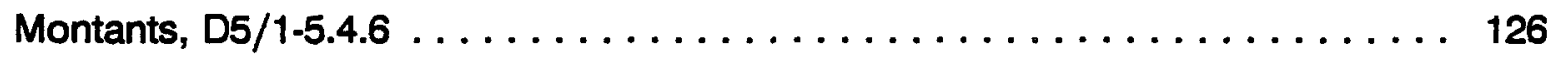

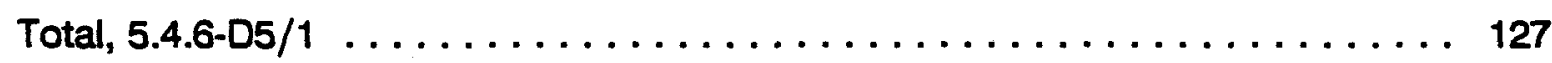

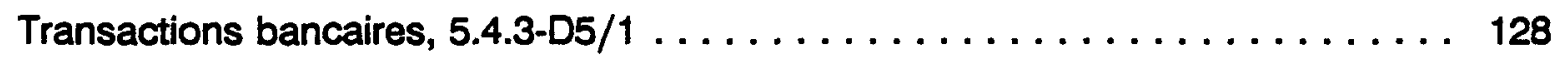

Transactions bancaires, $5.4 .3-D 5.4 / 3 \ldots \ldots \ldots \ldots \ldots \ldots \ldots \ldots \ldots \ldots 129$

Copie jaune chèque et talon, $05.4 / 1-5.4 .2 \ldots \ldots \ldots \ldots \ldots \ldots \ldots \ldots \ldots 130$

Chèques et dépôts en circulation, $D 5.4 / 2-5.4 .2 \ldots \ldots \ldots \ldots \ldots \ldots \ldots \ldots 131$

Chèques et dépôts en circulation, $5.4 .2-D 5.4 / 3 \ldots \ldots \ldots \ldots \ldots \ldots \ldots \ldots 132$

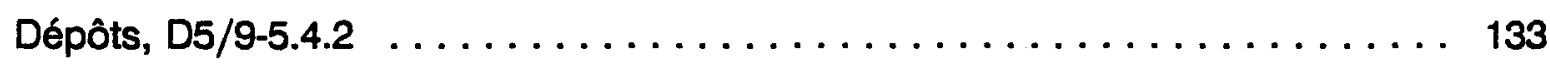

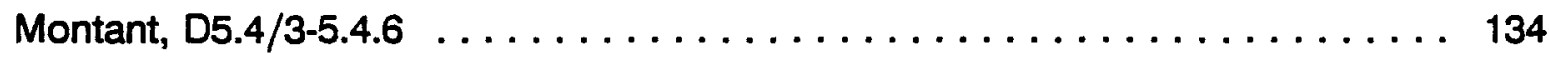

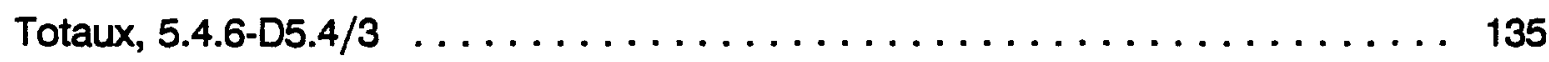




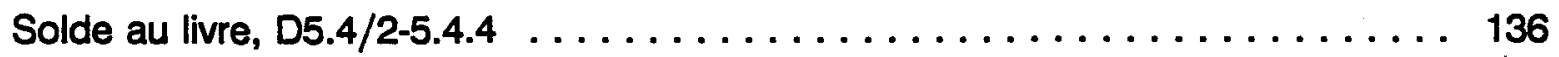

Total des chèques, $0-5.4 .4 \ldots \ldots \ldots \ldots \ldots \ldots \ldots \ldots \ldots \ldots \ldots \ldots \ldots$

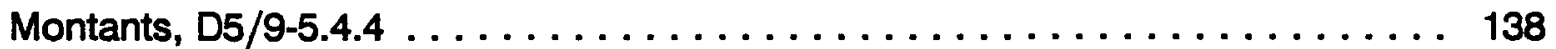

Solde au livre, $5.4 .4-D 5.4 / 3 \ldots \ldots \ldots \ldots \ldots \ldots \ldots \ldots \ldots \ldots \ldots \ldots \ldots . \ldots \ldots$

Solde, 5.4.4-5.4.5 ............................ 140

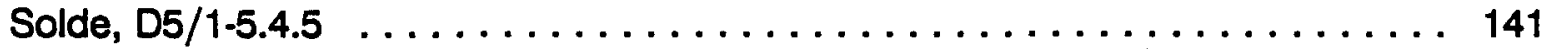

Formulaires de transactions, $D 5 / 8-e \ldots \ldots \ldots \ldots \ldots \ldots \ldots \ldots \ldots \ldots . \ldots \ldots$

Copies jaunes des chèques et talon, e-D5.4/1 $\ldots \ldots \ldots \ldots \ldots \ldots \ldots \ldots 143$

\section{Processus 5.5, Payer les factures des four-} nisseurs

Diagrammes de flux de données $\ldots \ldots \ldots \ldots \ldots \ldots \ldots \ldots$

5.5 Payer les factures des fournisseurs $\ldots \ldots \ldots \ldots \ldots \ldots \ldots \ldots \ldots \ldots 146$

Processus de traitement $\ldots \ldots \ldots \ldots \ldots \ldots \ldots \ldots \ldots \ldots \ldots \ldots$

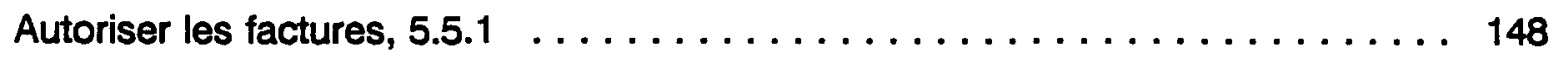

Sélectionner les factures qui seront payées, $5.5 .2 \ldots \ldots \ldots \ldots \ldots \ldots \ldots 149$

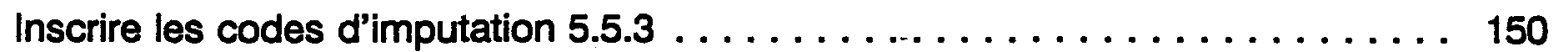

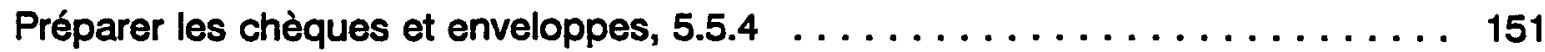

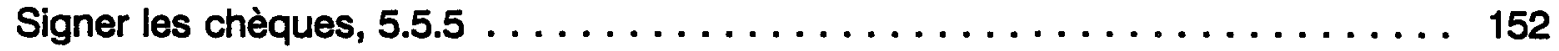

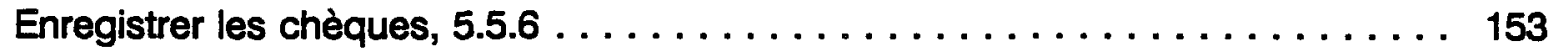

Faire un total de contrôle, $5.5 .7 \ldots \ldots \ldots \ldots \ldots \ldots \ldots \ldots \ldots \ldots \ldots$

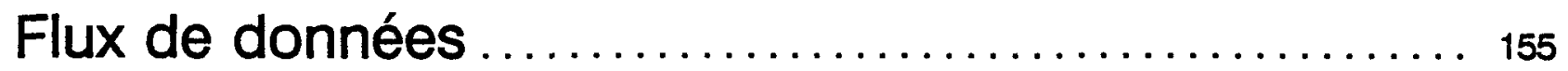

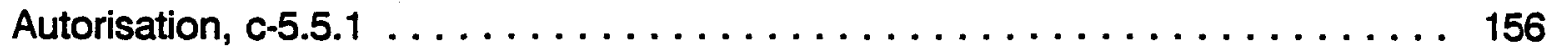

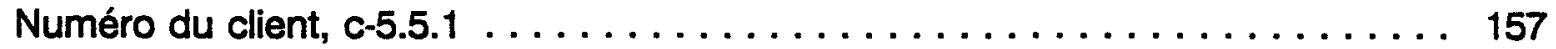

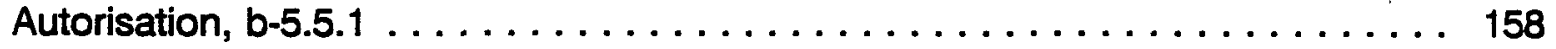

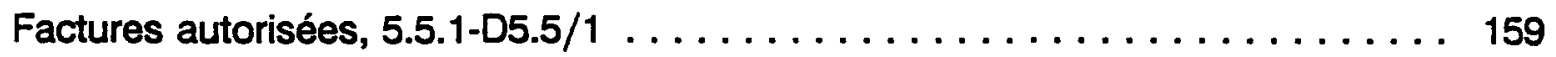

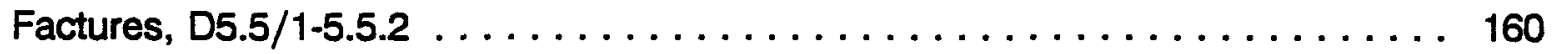


Numéro de compte, $05.5 / 3-5.5 .3 \ldots \ldots \ldots \ldots \ldots \ldots \ldots \ldots \ldots \ldots 1$

Numéro de compte, $05.5 / 4-5.5 .3 \ldots \ldots \ldots \ldots \ldots \ldots \ldots \ldots \ldots \ldots . \ldots \ldots 2$

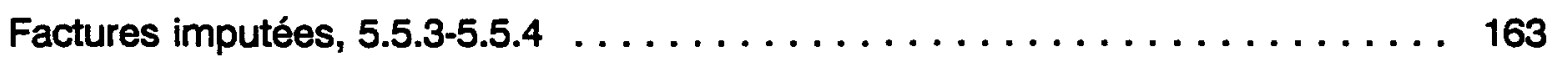

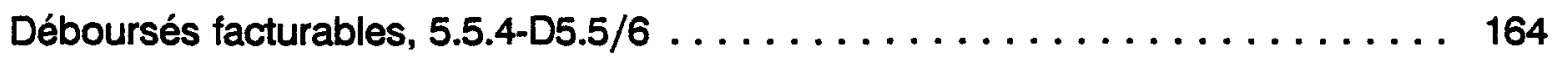

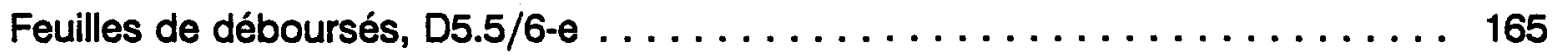

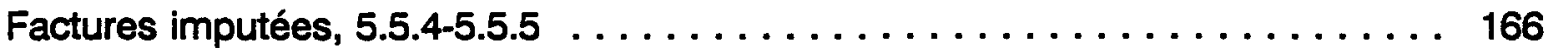

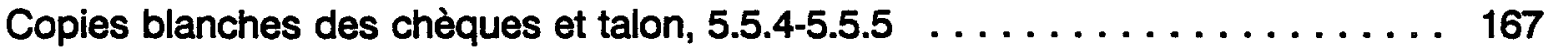

Copies blanches des chèques et talons, $5.5 .4-5.5 .5 \ldots \ldots \ldots \ldots \ldots \ldots \ldots 168$

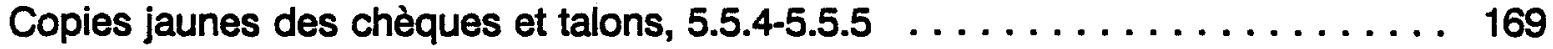

Copies jaunes des chèques et talons, $5.5 .6-5.5 .7 \ldots \ldots \ldots \ldots \ldots \ldots \ldots 170$

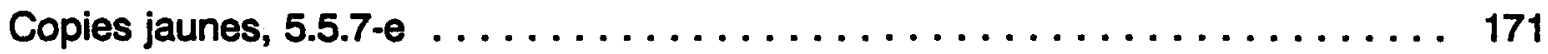

Total de contrôle, $5.5 .7-\theta \ldots \ldots \ldots \ldots \ldots \ldots \ldots \ldots \ldots \ldots \ldots \ldots . \ldots \ldots$

Chèques et pièces, $5.5 .5-d \ldots \ldots \ldots \ldots \ldots \ldots \ldots \ldots \ldots \ldots \ldots \ldots \ldots \ldots \ldots$

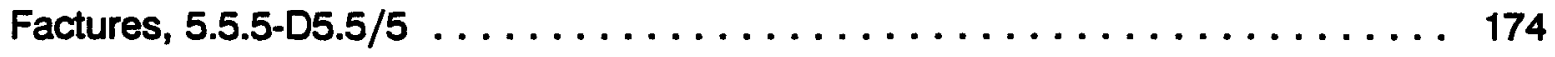

Copies blanches des chèques et talons, $5.5 .5-D 5.5 / 5 \ldots \ldots \ldots \ldots \ldots \ldots \ldots 175$

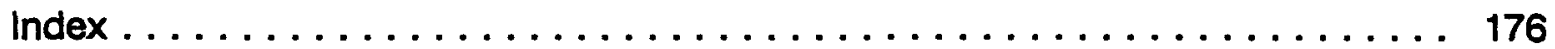




\section{Processus 2.0, Compiler le temps}


Diagrammes de flux de données 


\subsection{Compiler le temps}

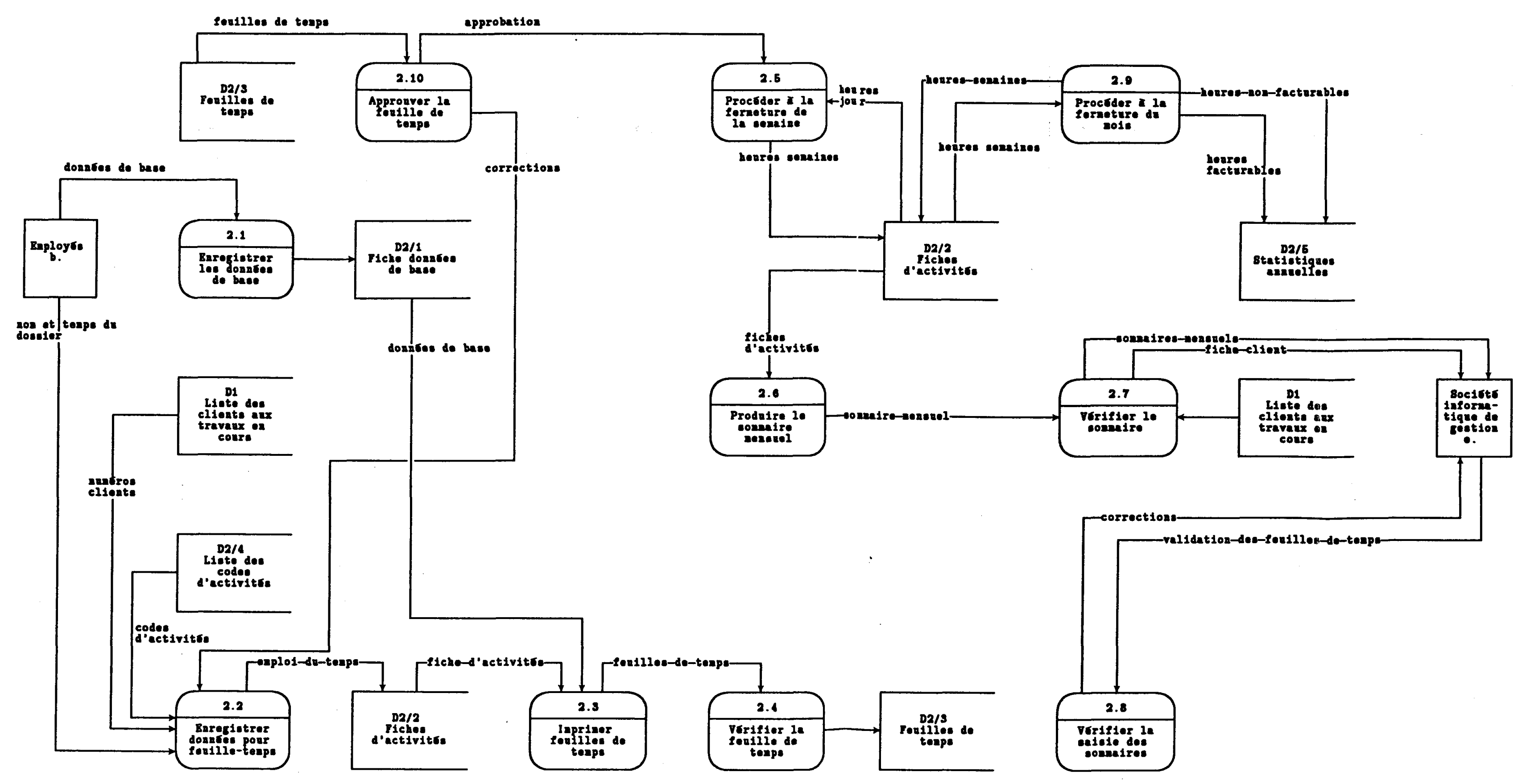




\subsection{Vérifier le sommaire}

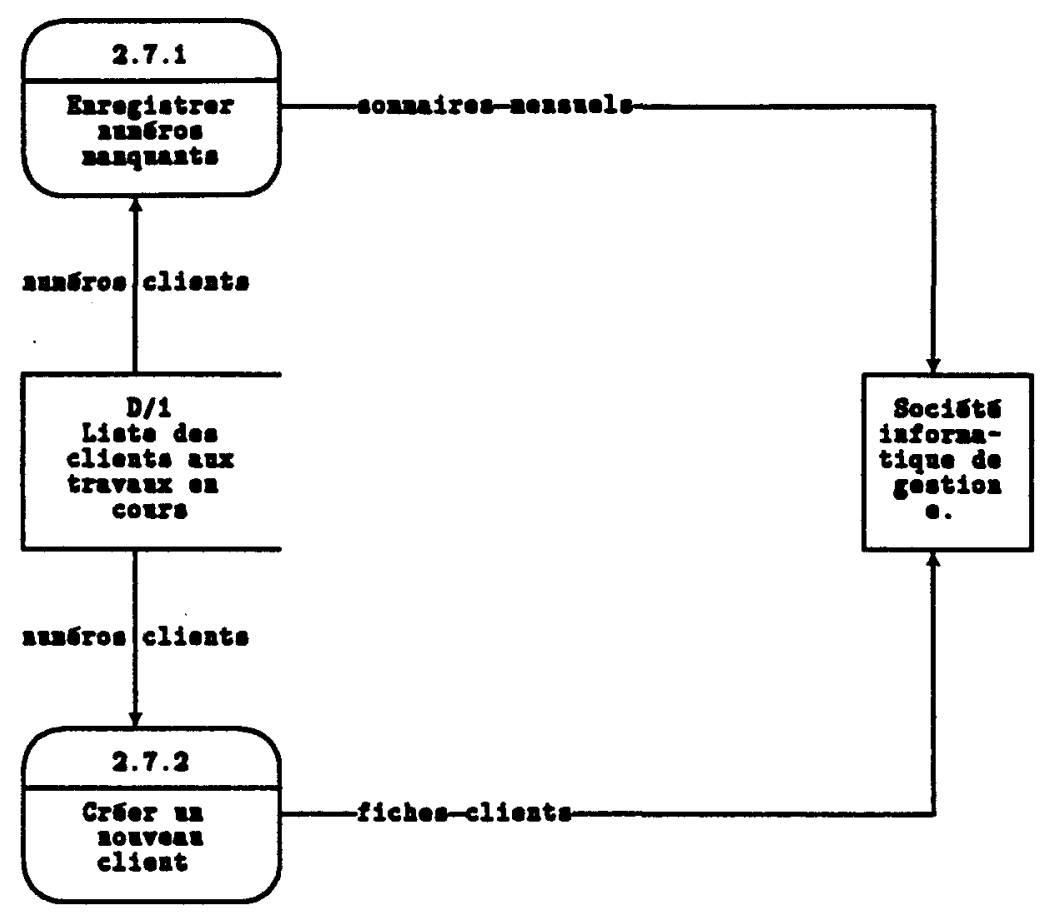


Processus de traitement 
Processus de traitement

\section{Enregistrer les données de base, 2.1}

\section{DESCRIPTION}

Enregistrer à toutes les semaines, à l'aide du programme de feuille de temps monté sur SYMPHONY (CHIFFRIER ÉLECTRONIQUE) les données de base nécessaires à la production des feuilles de temps.

\section{ENTRÉES}

Données de base, b-2.1
SORTIE

Données de base, 2.1-D2/1

\section{RÉSUMÉ DE LA LOGIQUE}

- enregistrer le nom de l'employé

- enregistrer le numéro de l'employé

- enregistrer la date de la feuille de temps

- enregistrer les heures régulières normales $(7 \mathrm{hres})$ de travail pour les journées allant de lundi à vendredi pour la feuille de temps qui sera produite. Ces heures serviront à calculer le temps supplémentaire. 
Processus de traitement

Enregistrer les données pour la feuille de temps, 2.2

\section{DESCRIPTION}

Enregistrer le temps, le code d'activités, le numéro du client pour chaque client/activités et chaque activité de la semaine.

\section{ENTRÉES}

Temps par dossier, b-2.2
SORTIE

Emploi du temps, 2.2-D2/2

\section{RÉSUMÉ DE LA LOGIQUE}

- Passer en mode recherche (F7F3)

- Enregistrer le nom du client ou le nom de l'activité (pour les activités non chargeables) pour lequel on recherche la fiche.

Si la fiche n'existe pas, enregistrer le nom de l'activité ou du client, enregistrer le numéro de l'activité et le nombre d'heures

à exécuter l'activité, et ce pour chaque jour de la semaine. 
Processus de traitement

\section{Imprimer la feuille de temps, 2.3}

\section{DESCRIPTION}

Prendre chaque fiche et procéder à l'impression en faisant une distinction entre le temps facturable et le temps non facturable. Sur la feuille de temps apparait pour chaque journée, le nombre d'heures associées à chaque activité, le nombre d'heures totales pour chacune des activités, le total des heures facturables, le total des heures non facturables, le total des heures et le nombre d'heures supplémentaires.

\section{ENTRÉES}

Fiches d'activité, D2/2 - 2.3
SORTIE

Feuille de $2.3-2.4$

\section{RÉSUMÉ DE LA LOGIQUE}

- $\quad$ imprimer nom de l'employé, numéro de l'employé, date de la feuille de temps Si code activité 99 et 740

imprimer nom du client

description du travail effectué

numéro du client

code de l'activité

heures totales

heures lundi

heures mardi

heures mercredi

heures jeudi

heures vendredi

heures samedi

total heures facturables

total heures facturables lundi

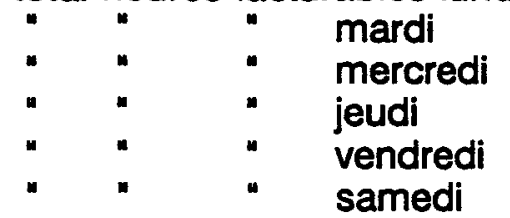




\section{Imprimer la feuille de temps (suite)}

si code d'activité 100 ou 740 imprimer:

nom de l'activité

code de l'activité

heures totales

heures lundi

heures mardi

heures mercredi

heures jeudi

heures vendredi

heures samedi

total heures non facturables

$"$ " " lundi

" " " mardi

" " " " mercredi

" " . " jeudi

" " " " vendredi

- " " samedi

total heures facturables et non facturables

total heures supplémentaires 


\section{Vérifier feuille de temps, 2.4}

\section{DESCRIPTION}

Vérifier la feuille de temps afin de s'assurer que toutes les journées ont été complétées.

\section{ENTRÉES}

Feuille de temps, $2.3-2.4$
SORTIE

Feuille de temps, $2.4-\mathrm{D} 2 / 3$

\section{RÉSUMÉ DE LA LOGIQUE}

- regarder si chaque journée $>7$ heures sinon, identifier la fiche activité pour laquelle il y a une erreur. 
Processus de traitement

\section{Procéder à la fermeture de la semaine, 2.5}

\section{DESCRIPTION}

Pour chaque fiche/activités, nous cumulons le nombre d'heures pour la semaine. Ce total sera utilisé pour produire le sommaire mensuel.

ENTRÉES

Heures jour, D2/2 - 2.5

Approbations, $2.10-2.5$
SORTIE

Heures semaines, $2.5-\mathrm{D} 2 / 2$

RÉSUMÉ DE LA LOGIQUE

Pour chaque fiche/activité, cumuler les heures pour les jours allant dudimanche au samedi. Inscrire le total de ces heures dans le cumulatif de la semaine.

Remettre le nombre d'heures pour chaque journée de la semaine en blanc. 
Processus de traitement

\section{Produire le sommaire mensuel, 2.6}

\section{DESCRIPTION}

A la fin de chaque mois, chaque employé produit son sommaire mensuel. Ce sommaire fournit de l'information sur les activités de l'employé durant le mois et sert à S.I.G. pour la mise à jour des travaux en cours.

\section{ENTRÉES}

Fiche d'activité, D2/2 - 2.6
SORTIE

Sommaire mensuel, $2.6 \cdot 2.7$

\section{RÉSUMÉ DE LA LOGIQUE}

Si le code d'activité 100 ou $=740$, inscrire

dans la section heures non facturables le nom de l'activité, le nombre d'heures pour la $1^{\text {èr }}$ semaine, la $2^{\text {ème }}$, la $3^{\text {ème }}$, la $4^{\text {ème }}$, la $5^{\text {ème }}$ semaine et le total du mois.

Si le code d'activités 100 et 740 , inscrire dans la section "heures facturables" le nom du client et le nombre d'heures pour la $1^{\text {ere }}, 2^{\text {eme }}, 3^{\text {ème }}, 4^{\text {ème }}$, et $5^{\text {eme }}$ semaine, ainsi que le total du mois.

Faire le total des heures facturables et non facturables pour chacune des semaines. Calculer temps supplémentaires pour chaque semaine. Calculer le nombre d'heures totales pour le mois et calculer le nombre d’heures en temps supplémentaire. 
Processus de traitement

\section{Vérifier le sommaire, 2.7}

\section{DESCRIPTION}

A chaque mois, la réceptionniste procède à la vérification des sommaires pour s'assurer qu'il n'y a pas d'erreurs au niveau des heures et des numéros de client.

\section{ENTRÉES}

Sommaires mensuels, $2.6 \cdot 2.7$
SORTIE

Sommaires mensuels, $2.7-1$

\section{RÉSUMÉ DE LA LOGIQUE}

Étant donné que les feuilles de temps ont été vérifiées et approuvées, il n'est pas supposé avoir d'erreurs sur les sommaires. Dans le cas où il y en aurait, les corrections sont effectuées directement sur la feuille du sommaire. 
Processus de traitement

\section{Enregistres les numéros manquants 2.7.1.}

\section{DESCRIPTION}

Sur le sommaire, inscrire les numéros de client manquants.

\section{ENTRÉES}

Sommaire mensuel, 2.6 - 2.7
SORTIE

Sommaires mensuels, 2.7.1-1

\section{RÉSUMÉ DE LA LOGIQUE}

Consulter la liste de clients aux travaux en cours (alphabétique), prendre note du numéro de client et l'inscrire sur le sommaire.

Si le client n'est pas sur la liste, créer un nouveau client. 
Processus de traitement

\section{Créer un nouveau client, 2.7 .2}

\section{DESCRIPTION}

II s'agit de créer un nouveau client lorsque le client n'apparaît pas dans la "liste des clients aux travaux en cours".

ENTRÉES

Numéros clients D1-2.7.2

RÉSUMÉ DE LA LOGIQUE

Regarder dans la liste de clients aux travaux en cours le numéro du client précédant l'endroit où le client sera situé.

Déterminer son numéro.

Compléter la fiche client.
SORTIE

Fiche client, 2.7.2-1 
Processus de traitement

\section{Vérifier la saisie des sommaires, 2.8}

\section{DESCRIPTION}

La vérification de la saisie des sommaires consiste à prendre le rapport nommé validation des feuilles de temps, et le vérifier les erreurs indiquées sur ce rapport. S'il y a effectivement une erreur, une correction est demandée.

\section{ENTRÉES}

Validation des feuilles de temps, $1-2.8$

\section{RÉSUMÉ DE LA LOGIQUE}

- regarder les messages d'erreurs sur le rapport

- regarder si erreur réelle, si oui

- demander correction
SORTIE

Corrections, 2.8-1 
Processus de traitement

\section{Procéder à la fermeture du mois, 2.9}

\section{DESCRIPTION}

La fermeture du mois consiste, pour chaque fiche-activité, à additionner le nombre d'heures mensuelles au cumulatif annuel d'heures facturables ou non facturables, selon le cas, et à remettre à zéro $(0)$, le nombre d'heures mensuelles, le nombre d'heures pour la $1^{\text {ore }}$, la $2^{2 m e}$, la $3^{\mathrm{dme}}$, la $4^{\mathrm{dme}}$ et la $5^{\mathrm{dme}}$ semaine.

ENTRÉES

Heures semaine, D2/2 - 2.9
SORTIE

Heures semaine, $2.9-\mathrm{D} 2 / 2$

Heures facturables, $2.9-\mathrm{D} 2 / 5$

Heures non facturables, $2.9 \cdot \mathrm{D} 2 / 5$

\section{RÉSUMÉ DE LA LOGIQUE}

Pour chaque fiche d'activités, si le code d'activités 99 et $F 740$, additionner le nombre d'heures mensuelles pour cette activité au nombre d'heures facturables pour le mois correspondant, remettre les champs suivants à zéro: le total du mois, les heures $1^{\text {tre }}, 2^{\text {eme }}, 3^{\text {eme }}, 4^{\text {ende }}$ et $5^{\text {eme }}$ semaine.

Si le code d'activité 100 ou $=740$, additionner le nombre d'heures mensuelles pour cette activité au nombre d'heures non facturables pour le mois correspondant, remettre à 0 les champs suivants: le total du mois, les heures pour la $1^{\text {dre }}$, la $2^{\text {dme }}$, la $3^{\text {dme }}$, la $4^{\text {eme }}$ et la $5^{\text {dme }}$ semaine. 
Processus de traitement

\section{Approuver la feuille de temps, 2.10}

\section{DESCRIPTION}

Approuver la répartition de l'emploi du temps entre le temps facturable et le temps non facturable. Approuver le temps qui est facturé pour les activités facturables.

\section{ENTRÉES}

Feuille de temps, D2/3 - 2.10
SORTIE

Approbation, $2.10-2.5$

Corrections, $2.10-2.2$

\section{RÉSUMÉ DE LA LOGIQUE}

Si non, approbation

Si oui, corrections

Vérifier la répartition du temps entre les activités facturables et les activités non-facturables. Si trop de temps non facturables faire des corrections.

Vérifier le temps facturé à chaque dossier apparaissant sur la feuille de temps. Si trop ou pas assez de temps facturé, faire des corrections.

Approuver la feuille de temps 
Flux de données 
Flux de données

\section{Nom et temps du dossier, b-2.2}

REF. SOURCE: $b$

REF. DESTIN.: 2.2
DESCRIPTION: Employés

DESCRIPTION: Enregistrer les données pour la feuille de temps

\section{DESCRIPTION DÉTAILLÉE:}

A chaque jour, l'employé note dans son agenda le nom du dossier sur lequel il a effectué du travail et le temps qu'il lui a consacré. Les périodes de temps sont compilées par quart d'heures.

\section{STRUCTURE DE DONNÉES INCLUSES:}

- nom du client

- temps noté en période entière d'une heure et en période de quart d'heures.

VOLUME:

Varie selon semaines. 
Flux de données

\section{Données de base, b-2.1}

REF. SOURCE: $b$

REF. DESTIN.: 2.1
DESCRIPTION: Employés

DESCRIPTION: Enregistrer données de base

DESCRIPTION DÉTAILLÉE:

Toutes les données statistiques nécessaires pour produire feuilles de temps.

STRUCTURE DE DONNÉES INCLUSES:

- nom de l'employé

- numéro d'employé

- code de l'activité principale

- date de la fin de la semaine

- nombre d'heures régulières/journée

VOLUME:

1 seule fiche/sem/employé. 
Flux de données

\section{Données de base, 2.1 - D2/1}

REF. SOURCE: 2.1

REF. DESTIN.: D2/1
DESCRIPTION: Enregistrer données de base

DESCRIPTION: Fiche données de base

\section{DESCRIPTION DÉTAILLÉE:}

Données statiques nécessaires pour produire les feuilles de temps.

STRUCTURE DE DONNÉES INCLUSES:

- nom de l'employé

- numéro de l'employé

- code de l'activité principale

- date de la fin de la semaine

- nombre d'heures régulières/journée

VOLUME:

1 seule fiche par employé par semaine 
Flux de données

\section{Fiche d'activité, d2/2 - 2.3}

REF. SOURCE: D2/2

REF. DESTIN.: 2.3
DESCRIPTION: Fiches d'activité

DESCRIPTION: Imprimer la feuille de temps

\section{DESCRIPTION DÉTAILLÉE:}

Détail pour une semaine des heures travaillées pour chaque fiche-activité et le cumulatif pour chaque semaine précédant la semaine courante. Le cumulatif pour les semaines suivant la courante est à zéro.

\section{STRUCTURE DE DONNÉES INCLUSES:}

- nom du client

- description du travail effectué

- numéro de code du client

- projet

- code d'activité

- total heures

- heures: dimanche à samedi

- cumulatif: $1^{\text {ere }}$ à $5^{\text {ème }}$ semaine

VOLUME:

Maximum 3000 . 
Flux de données

\section{Fiche d'activité, d2/2 - 2.6}

REF. SOURCE: D2/2

REF. DESTIN.: 2.6
DESCRIPTION: Fiches d'activité

DESCRIPTION: Produire le sommaire mensuel

\section{DESCRIPTION DÉTAILLÉE:}

Chaque fiche d'activité contient toute l'information pour produire le sommaire mensuel, sommaire qui est utilisé pour la mise à jour des travaux en cours.

Le nombre d'heures pour les journées allant du dimanche au samedi, pour chacune des fiches est à zéro. Les cumulatifs pour les semaines allant de 1 à 4 inclusivement sont supérieurs à zéro. Le cumulatif de la $5^{\text {ème }}$ semaine peut être différent de 0 , selon le nombre de semaines dans le mois (4 ou 5).

\section{STRUCTURE DE DONNÉES INCLUSES:}

- $\quad$ nom du client

- description du travail effectué

- numéro de code du client

- projet

- code d'activité

- total heures

- heures: dimanche à samedi

- cumulatif: $1^{\text {ere }}$ à $5^{\text {ème }}$ semaine

VOLUME:

Varie selon les employés. 
Flux de données

\section{Numéros de clients, d1 - 2.2}

REF. SOURCE: D1

REF. DESTIN.: 2.2
DESCRIPTION: Liste des clients aux travaux en cours

DESCRIPTION: Enregistrer les données pour la feuille de temps

DESCRIPTION DÉTAILLÉE:

Le numéro du client est celui qui est inscrit dans la liste des clients aux travaux en cours.

STRUCTURE DE DONNÉES INCLUSES:

- numéro du client: 8 chiffres

VOLUME:

1 par client. 
Flux de données

\section{Numéros de clients, d1 - 2.7.1}

REF. SOURCE: D1

REF. DESTIN.: 2.7.1
DESCRIPTION: Liste des clients aux travaux en cours

DESCRIPTION: Enregistrer numéros manquants

DESCRIPTION DÉTAILLÉE:

Le numéro du client retrouvé dans la liste de clients aux travaux en cours.

STRUCTURE DE DONNÉES INCLUSES:

- numéro du client: 8 chiffres

VOLUME:

Variable. 
Flux de données

Fiche client, 2.7.2-I

REF. SOURCE: 2.7 .2

DESCRIPTION: Créer un nouveau client

REF. DESTIN.: e

DESCRIPTION: Société d'Informatique de Gestion

DESCRIPTION DÉTAILLÉE:

Une fiche de client qui est utilisée pour créer un nouveau client ou le modifier.

STRUCTURE DE DONNÉES INCLUSES:

No:

Code: "C" pour création

" $M$ " pour modification

"A" pour annuler

No client: 8 chiffres

Nom du client: 73 caractères alpha-numériques

No de l'associé: 3 caractères numériques.

VOLUME:

20 par mois. 
Flux de données

\section{Corrections, $2.10-2.2$}

REF. SOURCE: 2.10

REF. DESTIN.: 2.2
DESCRIPTION: Approuver la feuille de temps

DESCRIPTION: Enregistrer les données pour la feuille de temps

\section{DESCRIPTION DÉTAILLÉE:}

Lorsqu'un employé produit sa feuille de temps, celle-ci doit être approuvée par son supérieur immédiat. Quelques fois, le supérieur immédiat demande à un employé de réduire le temps chargé dans le dossier pour diverses raisons.

STRUCTURE DE DONNÉES INCLUSES:

- nom du client

- nombre d'heures à facturer

VOLUME:

Variable. 
Flux de données

\section{Emploi du temps, 2.2 - D2/2}

REF. SOURCE: 2.2

DESCRIPTION: Enregistrer les données pour la feuille de temps

REF. DESTIN.: D2/2

DESCRIPTION: Fiches d'activités

DESCRIPTION DÉTAILLÉE:

Pour chaque fiche-activité, le temps travaillé est inscrit dans la journée correspondante.

STRUCTURE DE DONNÉES INCLUSES:

- nom du client

- numéro du client

- code d'activité

- temps/jour consacré à l'activité

VOLUME:

Variable. 
Flux de données

\section{Feuilles de temps, $2.3-2.4$}

REF. SOURCE: 2.3

REF. DESTIN.: 2.4
DESCRIPTION: Imprimer la feuille de temps

DESCRIPTION: Vérifier la feuille de temps

\section{DESCRIPTION DÉTAILLÉE:}

Contient toute l'information sur l'emploi du temps d'un employé pour une semaine donnée. A chaque semaine, l'employé produit une feuille de temps, dans certains cas, un employé peut produire plus d'une feuille de temps. Cela se produit lorsqu'un employé travaille pour Harel, Drouin et Ass. et pour Harel, Drouin Gestion-Conseils. Lorsqu'un employé facture à des taux horaires différents, il doit également produire plusieurs feuilles de temps.

\section{STRUCTURE DE DONNÉES INCLUSES:}

- nom de l'employé

- numéro de l'employé

- date de la feuille de temps

- le nom du client ou le nom de l'activité

- numéro de code de l'activité

- nombre d'heures pour chacune des activités

- total des heures pour la semaine et pour chaque journée

- total des heures supplémentaires pour la semaine et pour chaque journée.

VOLUME:

Environ 120 par mois. 
Flux de données

\section{Approbation, $2.10-2.5$}

REF. SOURCE: 2.10

DESCRIPTION: Approuver la feuille de temps

REF. DESTIN.: 2.5

DESCRIPTION: Procéder à la fermeture de la semaine

\section{DESCRIPTION DÉTAILLÉE:}

L'approbation provient du supérieur immédiat. Celui-ci accepte la répartition du temps de l'employé entre les activités facturables et les non facturables. De plus, le supérieur accepte le temps imputé à chacune des activités chargeables.

STRUCTURE DE DONNÉES INCLUSES:

oui ou non

VOLUME:

1 par semaine. 
Flux de données

\section{Heures/jour, d2/2 - 2.5}

REF. SOURCE: D2/2

REF. DESTIN.: 2.5
DESCRIPTION: Fiches d'activités

DESCRIPTION: Procéder à la fermeture de la semaine

\section{DESCRIPTION DÉTAILLÉE:}

II s'agit de lire dans chaque fiche-activités le nombre d'heures pour chaque journée.

STRUCTURE DE DONNÉES INCLUSES:

heures travaillées le dimanche

" " "lundi

" " " mardi

" " " "mercredi

" " "jeudi

" " vendredi

" " samedi

VOLUME: 
Flux de données

\section{Sommaire mensuel, $2.6-2.7$}

REF. SOURCE: 2.6

DESCRIPTION: Produire le sommaire mensuel

REF. DESTIN.: 2.7

DESCRIPTION: Vérifier le sommaire

\section{DESCRIPTION DÉTAILLÉE:}

Contient toute l'information nécessaire à la mise à jour de l'auxiliaire des travaux en cours. Pour chaque fiche-activité, le sommaire nous fournit le nombre d'heures travaillées par semaine et le total du mois. II nous indique également le temps supplémentaire par semaine et pour le mois.

STRUCTURE DE DONNÉES INCLUSES:

- numéro de l'employé

- nom de l'employé

- mois de sommaire

- nom de l'activité

- description travail effectué

- $\quad$ numéro du client

- numéro de code de l'activité

Pour chaque activité:

- le nombre d'heures totales pour le mois, pour la $1^{\text {dre }}$ a la $5^{\text {dme }}$ semaine;

- le total des heures facturables et non facturables pour le mois et chacune des semaines;

- le temps supplémentaire pour le mois et pour chacune des semaines.

VOLUME:

Environ 120 par mois. 
Flux de données

\section{Heures semaines, d2/2 - 2.9}

REF. SOURCE: D2/2

REF. DESTIN.: 2.9
DESCRIPTION: Fiches d'activités

DESCRIPTION: Procéder à la fermeture du mois

\section{DESCRIPTION DÉTAILÉE:}

Pour chaque fiche-activité, nous allons lire le nombre d'heures pour chacune des semaines.

STRUCTURE DE DONNÉES INCLUSES:

heures $1^{\text {dre }}$ à $5^{\text {dme }}$ semaine

VOLUME:

5 par fiche d'activité. 
Flux de données

\section{Heures semaines, $2.9-$ D2/2}

REF. SOURCE: 2.9

REF. DESTIN.: D2/2
DESCRIPTION: Procéder à la fermeture du mois DESCRIPTION: Fiches activités

DESCRIPTION DÉTAILLÉE:

Pour chaque fiche-activité, nous allons inscrire zéro $(0)$ pour la $1^{\text {èr }}$ à la $5^{\text {d̀me }}$ semaine.

STRUCTURE DE DONNÉES INCLUSES:

$$
\begin{aligned}
& \text { heures } 1^{\text {dre }} \text { semaine }=0 \\
& \text { " } 3^{\text {ome }} "=0 \\
& \text { - } 4^{\mathrm{ème}} \cdot=0 \\
& \text { - } 5^{\mathrm{sm \theta}} \cdot=0
\end{aligned}
$$

VOLUME:

5 par fiche. 
Flux de données

\section{Feuille de temps, d2/3 - 2.10}

REF. SOURCE: D2/3

REF. DESTIN.: 2.10
DESCRIPTION: Feuilles de temps

DESCRIPTION: Approuver feuilles de temps

\section{DESCRIPTION DÉTAILLÉE:}

Contient toute l'information sur l'emploi du temps d'un employé pour une semaine donnée.

\section{STRUCTURE DE DONNÉES INCLUSES:}

- nom de l'employé

- numéro de l'employé

- date de la feuille de temps

- nom du client ou de l'activité

- numéro de code de l'activité

- nombre total d'heures pour chaque activité

- nombre d'heures par jour pour chaque activité

- total des heures pour la semaine et pour chaque journée

- total des heures supplémentaires pour la semaine et pour chaque journée.

VOLUME:

1 par employé. 
Flux de données

\section{Heures facturables et non facturables $2.9-$ D2/5}

REF. SOURCE: 2.9

REF. DESTIN.: D2/5
DESCRIPTION: Procéder à la fermeture du mois

DESCRIPTION: Statistiques annuelles

DESCRIPTION DÉTAILLÉE:

Le cumulatif des heures facturables et des heures non facturables pour le mois courant.

STRUCTURE DE DONNÉES INCLUSES:

- total heures facturables

- total heures non-facturables

VOLUME:

1 fois par mois. 
Flux de données

\section{Validation des feuilles de temps, e-2.8}

REF. SOURCE: $e$

REF. DESTIN.: 2.8
DESCRIPTION: Société d'Informatique de Gestion

DESCRIPTION: Vérifier la saisie des feuilles de temps

\section{DESCRIPTION DÉTAILLÉE:}

La liste de toutes les transactions de feuilles de temps pour lesquelles une erreur a été identifiée. Les erreurs sont identifiées par des étoiles ou par un message sous la ligne de la transaction.

STRUCTURE DE DONNÉES INCLUSES:

- numéro de la transaction

- numéro d'employé

- date de la transaction

- numéro du client

- code d'activités

- nombre d'heures pour l'activité

- nombre d'heures facturables

- nombre d'heures non facturables

- heures totales

- heures supplémentaires

- type de transaction

VOLUME:

Environ 200. 
Flux de données

\section{Corrections, 2.8-e}

REF. SOURCE: 2.8

REF. DESTIN.: e
DESCRIPTION: Vérifier la saisie des sommaires

DESCRIPTION: Société d'Informatique de Gestion

\section{DESCRIPTION DÉTAILLÉE:}

La correction est indiquée sur le rapport "validation des feuilles de temps". La correction peut être au niveau du numéro de client, du code d'activité, du nombre d'heures pour l'activité, du nombre d'heures pour le temps facturable, du nombre d'heures pour le temps non-facturable.

STRUCTURE DE DONNÉES INCLUSES:

Une ou plusieurs des champs suivants:

- numéro du client

- code d'activités

- heures facturables

- heures non facturables

VOLUME:

Variable. 
Processus 5.1, Enregistrer les ventes 
Diagrammes de flux de données 


\subsection{Enregistrer les ventes}

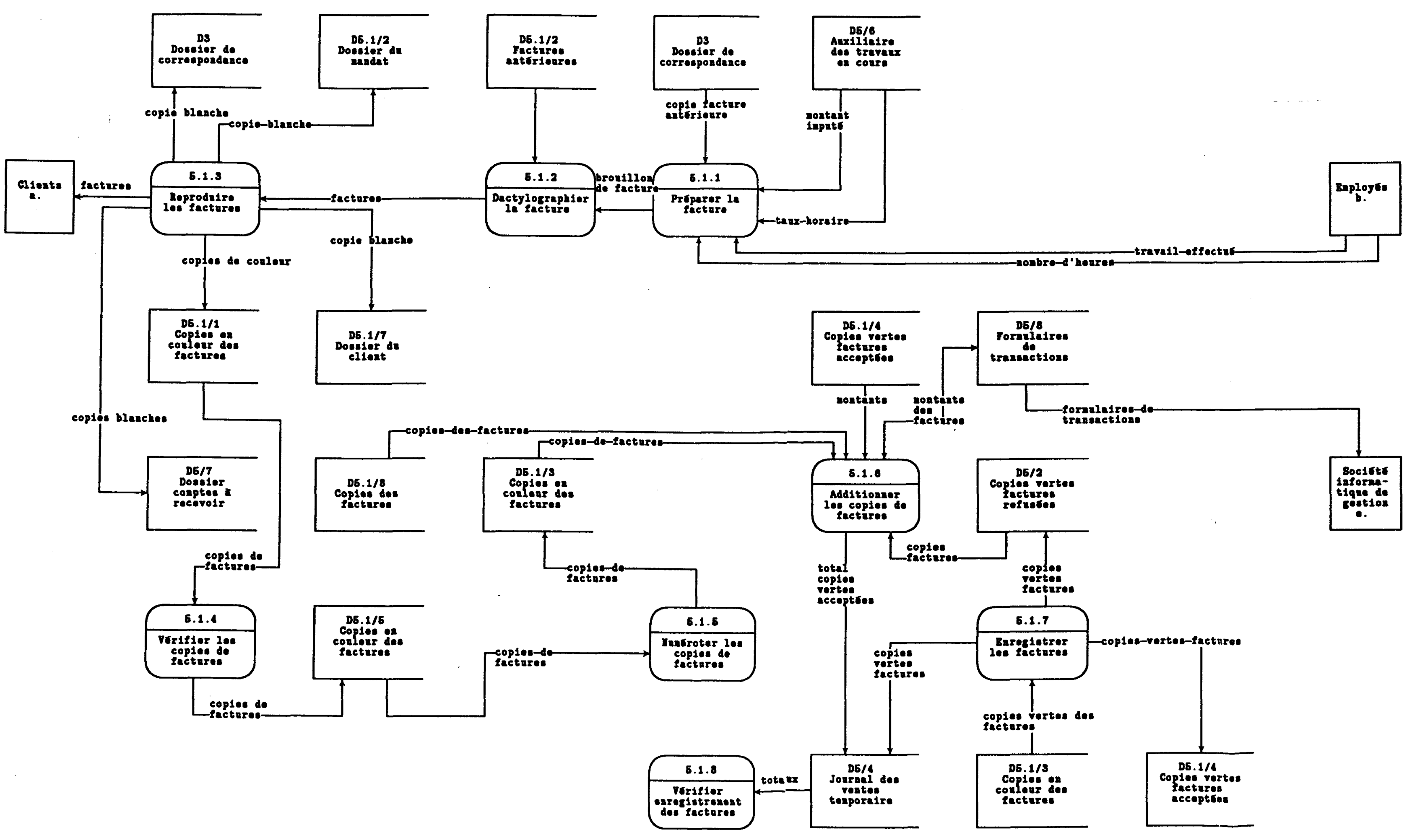


Processus de traitement 
Processus de traitement

\section{Préparer la facture, 5.1.1}

\section{DESCRIPTION}

Pour préparer la facture, l'associé ou le vérificateur senior prend l'auxiliaire des travaux en cours pour connaître le montant imputé au dossier de son client. Étant donné que l'auxiliaire des travaux en cours est mis à jour le 30 du mois précédent, l'associé ou le vérificateur senior doit demander aux personnes de son équipe le temps travaillé sur le dossier entre le 30 du mois précédent jusqu'au jour de la facturation. Par la suite, la facture est dictée ou des corrections sont apportées sur la copie de la facture antérieure.

\section{ENTRÉES}

Copie facture antérieure, D3-5.1.1

Montant imputé, D5/6-5.1.1

Taux horaires, D5/6-5.1.1

Nombre d'heures, b-5.1.1

Travail effectué, b-5.1.1

\section{SORTIE}

Brouillon de facture,

$5.1 .1-5.1 .2$

\section{RÉSUMÉ DE LA LOGIQUE}

- regarder l'auxiliaire des travaux en cours, prendre note des montants imputés au dossier

- demander aux employés le temps travaillé sur le dossier depuis le lendemain de la mise à jour des travaux en cours

- regarder, dans l'auxiliaire des travaux en cours, le taux horaire

- si Harel, Drouin \& Associés

si ancien client

- dicter le contenu de la facture ou

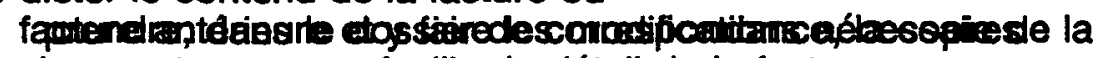
sinon noter sur une feuille de détail de la facture

de lasta Drouin Gestion Conseil noter sur une feuille le détail 
Processus de traitement

\section{Dactylographier la facture, 5.1.2}

\section{DESCRIPTION}

Il s'agit ici, selon le cas, à l'aide d'un appareil de traitement de textes, de modifier l'information contenue sur une facture antérieure ou de dactylographier le contenu de la facture.

ENTRÉES

Facture antérieure, D5.1/2-5.1.2

Brouillon de facture, 5.1.1-5.1.2
SORTIE

Copie de la facture courante

5.1.2-D3

RÉSUMÉ DE LA LOGIQUE:

- si facture de Harel, Drouin et Associés

- si ancien client

- retrouver la facture précédente

- faire les modifications nécessaires

- imprimer la facture

- reproduire la facture

- mettre la copie dans le dossier de correspondance

- si nouveau client

- dactylographier la facture

- reproduire la facture

- mettre la copie dans dossier de correspondance

- si facture de Harel Drouin Gestion Conseil

- dactylographier la facture

- reproduire la facture

- mettre la facture dans le dossier de correspondance 
Processus de traitement

\section{Reproduire les factures, 5.1.3}

\section{DESCRIPTION}

Une fois que les factures ont été dactylographiées, ces dernières sont reproduites sur des feuilles blanches et sur des feuilles de couleur.

ENTREES

Factures 5.1.2-5.1.3

\section{SORTIE}

Copies blanches, 5.1.3-D3 Copies de couleur, 5.1.3-D5.1/1 Copie blanche, 5.1.3-D5/7 Copie blanche, 5.1.3-D5.1/6 Copie blanche, 5.1.3-D5.1/7

Factures 5.1.3-a

\section{RÉSUMÉDE LALOGIQUE:}

- reproduire toutes les factures sur du papier blanc Harel Drouin Gestion Conseil

Pierre-Paul Morin 1 copie blanche Jean-Guy Grenier 2 copies blanches

Gérald Bélanger 1 copie blanche

- si facture de Harel, Drouin et Associés, placer la copie blanche dans le dossier de correspondance

sinon

si facture de Pierre-Paul Morin placer la copie blanche dans le dossier du mandat si facture de Jean-Guy Grenier placer une copie blanche dans dossier du client placer autre copie blanche dans dossier comptes à recevoir

si facture de Gérald Bélanger placer copie blanche dans dossier de compte à recevoir

- si facture Harel, Drouin et Associés reproduire les factures sur du papier vert

- si facture de Harel Drouin Gestion Conseil

si facture de Pierre-Paul Morin reproduire les factures sur du papier rose si facture de Jean-Guy Grenier reproduire les factures sur du papier bleu si facture de Gérald Bélanger reproduire les factures sur du papier jaune 
Processus de traitement

\section{Vérifier les copies de factures, 5.1.4}

\section{DESCRIPTION}

Prendre toutes les copies de couleur des factures et mettre en évidence le montant de la facture. Si la facture contient plus d'une page, reporter le montant de la facture sur la première page.

ENTRÉES

Copies de factures, $D 5.1 / 1,-5.1 .4$
SORTIE

Copies de factures, 5.1.4D5.1.5

RÉSUMÉ DE LA LOGIQUE:

- si la facture a une seule page encercler le montant de la facture

- $\quad$ sinon reporter le montant de la facture sur la première page 
Processus de traitement

Numéroter les copies de factures, 5.1.5

\section{DESCRIPTION}

Regrouper ensemble les factures vertes, les roses, les jaunes et les bleues. Numéroter séquentiellement avec des numérateurs différents les factures vertes, roses, jaunes et bleues.

\section{ENTRÉES}

Copies de factures, D5.1/5-5.1.5
SORTIE

Copies de factures, 5.1.4-

D5.1/5

- $\quad$ si factures vertes, prendre le numérateur de copies de Harel, Drouin et Associés et numéroter les copies de factures

si factures roses, jaunes ou bleues, prendre le cachet de Harel Drouin Gestion Conseil et numéroter les copies de factures 
Processus de traitement

\section{Additionner le copies de factures, 5.1.6}

\section{DESCRIPTION}

Prendre toutes les copies vertes enregistrées dans le journal des ventes, additionner les montants et l'inscrire dans le journal des ventes. Prendre toutes les copies vertes rejetées, en additionner les montants et inscrire ce montant avec le numéro de compte sur un formulaire de transactions. Prendre toutes les copies roses, jaunes et vertes, additionner les montants et inscrire le résultat de l'addition sur le formulaire de transactions.

\section{ENTRÉES}

Copies de factures, D5.1/3-5.1.6

Montants, D5.1/4-5.1.6

Copies de factures, D5/2-5.1.6
SORTIE

Montants des factures, 5.1.6

D5/8

Copies des factures, 5.1.6

$D 5,1 / 8$

Total copies vertes acceptées

5.1.6-D5/4

\section{RÉSUMÉ DE LA LOGIQUE}

- prendre copies vertes factures acceptées

- additionner les factures

- enregistrer montant et numéro de compte dans "journal des ventes temporaire"

- prendre copies vertes factures refusées

- additionner les factures

- inscrire le numéro de compte et le montant sur un formulaire de transactions

- prendre copies roses, jaunes et bleues

- additionner les factures

- inscrire le numéro de compte et le montant sur un formulaire de transaction 
Processus de traitement

\section{Enregistrer les factures, 5.1.7}

\section{DESCRIPTION}

Une personne du département de la comptabilité va à la Société d'informatique de gestion (S.I.G.) et enregistre les copies vertes des factures dans le "journal des ventes temporaire". Fréquemment, le système informatique de S.I.G. refuse certaines factures en mentionnant que le client n'existe pas. L'enregistrement des factures produit deux groupes de factures, les factures acceptées par le système et les factures rejetées.

ENTRÉES

Copies vertes des factures, D5.1/35.1.7
SORTIE

Copies vertes des factures, 5.1.7-D5/2

Copies vertes des factures,

5.1.7-D5/4

Copies vertes des factures,

5.1.7-D5.1/4

\section{RÉSUMÉ DE LA LOGIQUE}

- taper le numéro du client

- si client existe, enregistrer: - numéro de la facture

- la date de la facture

- le nom du client

- le montant de la facture

- si client n'existe pas

- mettre la facture de côté 
Processus de traitement

\section{Vérifier l'enregistrement des factures, 5.1.8}

\section{DESCRIPTION}

Vérifier le "journal des ventes temporaire" afin de s'assurer que les écritures balancent. Si les écritures ne balancent pas, trouver et corriger l'erreur.

\section{ENTRÉES}

Totaux D5/4-5.1.8

RÉSUMÉ DE LA LOGIQUE

- regarder si les transactions enregistrées balancent

si oui, ne rien faire

si non, trouver l'erreur (''addition ou l'enregistrement)
SORTIE

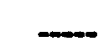


Flux de données 
Flux de données

\section{Totaux, D5/4-5.1.8}

REF. SOURCE: D5/4

DESCRIPTION: "Journal des ventes temporaire"

REF. DESTIN.: 5.1.8

DESCRIPTION: vérifier l'enregistrement des factures

\section{DESCRIPTION DÉTAILLÉE:}

Total des crédits et des débits enregistrés dans le "journal des ventes temporaire."

STRUCTURE DE DONNÉES INCLUSES:

2 chiffres.

VOLUME:

2 chiffres par mois. 
Flux de données

\section{Total copies vertes acceptées}

REF. SOURCE: 5.1.6

REF. DESTIN.: D5/4
DESCRIPTION: Additionner les copies de factures

DESCRIPTION: "Journal des ventes temporaire"

DESCRIPTION DÉTAILLÉE:

Total de toutes les factures qui ont été acceptées par le système informatique.

STRUCTURE DE DONNÉES INCLUSES:

Chiffre.

VOLUME:

1 par mois. 
Flux de données

\section{Copies vertes factures, 5.1.7-D5/2}

REF. SOURCE: $\mathbf{5 . 1 . 7}$

REF. DESTIN.: D5/2
DESCRIPTION: Enregistrer les factures

DESCRIPTION: Copies vertes factures refusées

\section{DESCRIPTION DÉTAILLÉE:}

Contient le nom du client, l'adresse, le nom du contact, le numéro du client, la description du travail effectué et le montant. Circulent dans ce flux seulement les factures qui ont été refusées par le système informatique lors de l'opération "enregistrer les factures".

STRUCTURE DE DONNÉES INCLUSES:

Nom du client

Adresse du client (no, rue, numéro de bureau, ville, province, code postal)

Nom du contact: à qui est destinée la facture

Description du travail effectué

Le montant de la facture

VOLUME:

Environ 100 
Flux de données

\section{Copies vertes factures, 5.1.7-D5.1/4}

REF. SOURCE: 5.1.7

REF. DESTIN.: D5.1/4
DESCRIPTION: Enregistrer les factures

DESCRIPTION: Copies vertes factures acceptées

\section{DESCRIPTION DÉTAILLÉE:}

Contient le nom du client, l'adresse, le nom du contact, le numéro du client, la description du travail effectué et le montant. Circulent dans ce flux seulement les factures qui ont été acceptées par le système informatique lors de l'opération "enregistrer les factures".

STRUCTURE DE DONNÉES INCLUSES:

Nom du client

Adresse du client (no, rue, numéro de bureau, ville, province, code postal)

Nom du contact: à qui est destinée la facture

Description du travail effectué

Le montant de la facture

VOLUME:

Environ 260 
Flux de données

\section{Copies factures, D5/2-5.1.6}

REF. SOURCE: D5/2

DESCRIPTION: Copies vertes factures refusées

REF. DESTIN.: 5.1.6

DESCRIPTION: Additionner les copies de factures

\section{DESCRIPTION DÉTAILLÉE:}

Contient le nom du client, l'adresse, le nom du contact, le numéro du client, la description du travail effectué et le montant. Circulent dans ce flux seulement les factures qui ont été refusées par le système informatique lors de l'opération "enregistrement des factures".

STRUCTURE DE DONNÉES INCLUSES:

Nom du client

Adresse du client (no, rue, numéro de bureau, ville, province, code postal)

Nom du contact: à qui est destinée la facture

Description du travail effectué

Le montant de la facture

VOLUME:

environ 100. 
Flux de données

\section{Formulaires de transactions, D5/8-e}

REF. SOURCE: D5/8

DESCRIPTION: Formulaire de transactions

REF. DESTIN.: e

DESCRIPTION: Société d'informatique de gestion

DESCRIPTION DÉTAILLÉE:

Comprend les formulaires qui servent à inscrire différentes écritures de journal. Ces formulaires sont envoyés à la Société d'informatique afin qu'elle mette à jour les différents livres comptables et auxiliaires.

STRUCTURE DE DONNÉES INCLUSES:

Un formulaire contient généralement 4 écritures comptables ( 1 pour les factures vertes rejetées, 1 pour les factures jaunes, 1 pour les factures bleues et 1 pour les factures roses), numéro de compte, numéro de sous compte, montant, description.

VOLUME:

1 par mois. 
Flux de données

\section{Formulaires de transaction, 5.1.6-D5/8}

REF. SOURCE: 5.1 .6

DESCRIPTION: Additionner les copies de factures

REF. DESTIN.: D5/8

DESCRIPTION: Société d'informatique de gestion

DESCRIPTION DÉTAILLÉE:

Contient le total des copies roses, le total des copies jaunes, le total des copies bleues des factures de Harel Drouin Gestion Conseil et le total des copies vertes des factures rejetées de Harel, Drouin et Associés.

STRUCTURE DE DONNÉES INCLUSES:

Numéro de compte, numéro du sous compte, montant, description (honoraires mois année).

VOLUME:

4 par mois. 
Flux de données

\section{Copies des factures, 5.1.6-D5.1/8}

REF. SOURCE: 5.1 .6

DESCRIPTION: Additionner les copies de factures

REF. DESTIN.: D5.1/8

DESCRIPTION: Copies des factures

DESCRIPTION DÉTAILLÉE:

Contient toutes les copies numérotées des factures de Harel Drouin Gestion Conseil (jaunes, roses et bleues) et les copies vertes numérotées qui ont été refusées par le système informatique lors de l'opération "enregistrer les factures".

STRUCTURE DE DONNÉES INCLUSES:

- copies vertes numérotées qui ont été refusées par le système informatique lors de l'opération "enregistrer les factures"

- les copies de factures jaunes, roses et bleues numérotées

Référer au flux de données 5.1.5-D5.1/3 pour connaître le contenu d'une copie de factures.

VOLUME: environ 150. 
Flux de données

\section{Copies des factures, D5.1/8-e}

REF. SOURCE: 5.1.6

REF. DESTIN.: e
DESCRIPTION: Copies des factures

DESCRIPTION: Société d'informatique de gestion

DESCRIPTION DÉTAILLÉE:

Contient toutes les copies numérotées des factures de Harel Drouin Gestion Conseil (jaunes, roses et bleues) et les copies vertes numérotées qui ont été refusées par le système informatique lors de l'opération "enregistrer les factures".

STRUCTURE DE DONNÉES INCLUSES:

- copies vertes numérotées qui ont été refusées par le système informatique lors de l'opération "enregistrer les factures"

- les copies de factures jaunes, roses et bleues numérotées

Référer au flux de données 5.1.5-D5.1/3 pour connaître le contenu d'une copie de factures.

VOLUME:

Environ 150. 
Flux de données

\section{Montants, D5.1/4-5.1.6}

REF. SOURCE: D5.1/4

REF. DESTIN.: 5.1.6
DESCRIPTION: Copies vertes factures acceptées DESCRIPTION: Additionner les copies de factures

DESCRIPTION DÉTAILLÉE:

Montants inscrits sur la première page de la facture.

STRUCTURE DE DONNÉES INCLUSES:

Montant de la facture.

VOLUME:

Environ 250. 
Flux de données

\section{Copies de factures, D5.1/3-5.1.6}

REF. SOURCE: D5.1/3

DESCRIPTION: Copies en couleur des factures

REF. DESTIN.: 5.1.6

DESCRIPTION: Additionner les copies de factures

\section{DESCRIPTION DÉTAILLÉE:}

Contient le nom du client, l'adresse, le nom du contact, le numéro du client, la description du travail effectué et le montant. Circulent dans ce flux de données les copies roses, bleues et jaunes des factures de Harel Drouin Gestion Conseil.

\section{STRUCTURE DE DONNÉES INCLUSES:}

Nom du client

adresse du client (no, rue, numéro bureau, ville, province, code postal)

nom du contact: à qui est destinée la facture

description du travail effectué

le montant de la facture

VOLUME:

Environ 200 par mois. 
Flux de données

\section{Copies vertes des factures D5.1/3-5.1.7}

REF. SOURCE: D5.1/3

REF. DESTIN.: 5.1.7
DESCRIPTION: Copies en couleur des factures

DESCRIPTION: Enregistrer les factures

DESCRIPTION DÉTAILLÉE:

Contient le nom du client, l'adresse, le nom du contact, le numéro du client, la description du travail effectué et le montant.

STRUCTURE DE DONNÉES INCLUSES:

Nom du client adresse du client (no, rue, numéro bureau, ville, province, code postal) nom du contact: à qui est destinée la facture description du travail effectué

le montant de la facture

VOLUME:

Environ 400 par mois. 
Flux de données

\section{Copies des factures, 5.1.5-D5.1/3}

REF. SOURCE: 5.1 .5

DESCRIPTION: Numéroter les copies de factures

REF. DESTIN.: D5.1/3

DESCRIPTION: Copies en couleur des factures

\section{DESCRIPTION DÉTAILLÉE:}

Contient le nom du client, l'adresse, le nom du contact, le numéro du client, la description du travail effectué et le montant.

. copies vertes: Harel, Drouin et associés

. copies roses: Harel Drouin Gestion Conseil, Pierre-Paul Morin

- copies bleues: Harel Drouin Gestion Conseil, Jean-Guy Grenier

. copies jaunes: Harel Drouin Gestion Conseil, Gérald Bélanger

STRUCTURE DE DONNÉES INCLUSES:

Nom du client

adresse du client (no, rue, numéro bureau, ville, province, code postal)

nom du contact: à qui est destinée la facture

description du travail effectué

le montant de la facture

VOLUME:

Environ 400 par mois. 
Flux de données

\section{Copies des factures, D5.1/5-5.1.5}

REF. SOURCE: D5.1/5

REF. DESTIN.: 5.1 .5
DESCRIPTION: Copies en couleur des factures

DESCRIPTION: Numéroter les copies de factures

\section{DESCRIPTION DÉTAILLÉE:}

Contient le nom du client, l'adresse, le nom du contact, le numéro du client, la description du travail effectué et le montant.

. copies vertes: Harel, Drouin et associés

. copies roses: Harel Drouin Gestion Conseil, Pierre-Paul Morin

. copies bleues: Harel Drouin Gestion Conseil, Jean-Guy Grenier

. copies jaunes: Harel Drouin Gestion Conseil, Gérald Bélanger

\section{STRUCTURE DE DONNÉES INCLUSES:}

Nom du client

adresse du client (no, rue, numéro bureau, ville, province, code postal)

nom du contact: à qui est destinée la facture

description du travail effectué

le montant de la facture

\section{VOLUME:}

Environ 400 par mois. 
Flux de données

\section{Copies des factures, 5.1.4-D5.1/5}

REF. SOURCE: 5.1 .4

REF. DESTIN.: D5.1/5
DESCRIPTION: Vérifier les copies de factures

DESCRIPTION: Copies en couleur des factures

\section{DESCRIPTION DÉTAILLÉE:}

Contient le nom du client, l'adresse, le nom du contact, le numéro du client, la description du travail effectué et le montant.

. copies vertes: Harel, Drouin et associés

- copies roses: Harel Drouin Gestion Conseil, Pierre-Paul Morin

. copies bleues: Harel Drouin Gestion Conseil, Jean-Guy Grenier

. copies jaunes: Harel Drouin Gestion Conseil, Gérald Bélanger

\section{STRUCTURE DE DONNÉES INCLUSES:}

Nom du client adresse du client (no, rue, numéro bureau, ville, province, code postal)

nom du contact: à qui est destinée la facture description du travail effectué

le montant de la facture

VOLUME:

Environ 400 par mois. 
Flux de données

\section{Copies des factures, D5.1/1-5.1.4}

REF. SOURCE: D5.1/1

DESCRIPTION: Copies en couleur des factures

REF. DESTIN.: 5.1.4

DESCRIPTION: Vérifier les copies factures

\section{DESCRIPTION DÉTAILLÉE:}

Contient le nom du client, l'adresse, le nom du contact, le numéro du client, la description du travail effectué et le montant.

. copies vertes: Harel, Drouin et associés

. copies roses: Harel Drouin Gestion Conseil, Pierre-Paul Morin

. copies bleues: Harel Drouin Gestion Conseil, Jean-Guy Grenier

- copies jaunes: Harel Drouin Gestion Conseil, Gérald Bélanger

STRUCTURE DE DONNÉES INCLUSES:

Nom du client

adresse du client (no, rue, numéro bureau, ville, province, code postal)

nom du contact: à qui est destinée la facture

description du travail effectué

le montant de la facture

VOLUME:

Environ $\mathbf{4 0 0}$ par mois. 
Flux de données

\section{Copie blanche, 5.1.3-D5/7}

REF. SOURCE: 5.1.3

REF. DESTIN.: D5/7
DESCRIPTION: Reproduire les factures

DESCRIPTION: Dossier du client

\section{DESCRIPTION DÉTAILLÉE:}

Contient le nom du client, l'adresse, le nom du contact, le numéro du client, la description du travail effectué et le montant. Cette copie, qui va dans le dossier du client est utilisée seulement par Jean-Guy Grenier de Harel Drouin Gestion Conseil.

\section{STRUCTURE DE DONNÉES INCLUSES:}

Nom du client

adresse du client (no, rue, numéro bureau, ville, province, code postal) nom du contact: à qui est destinée la facture description du travail effectué

le montant de la facture

VOLUME:

Environ 20 par mois. 
Flux de données

\section{Copie blanche, 5.1.3-D5/6}

REF. SOURCE: 5.1 .3

REF. DESTIN.: D5/6
DESCRIPTION: Reproduire les factures

DESCRIPTION: Dossier du mandat

\section{DESCRIPTION DÉTAILLÉE:}

Contient le nom du client, l'adresse, le nom du contact, le numéro du client, la description du travail effectué et le montant. Cette copie, qui va dans le dossier du mandat est utilisée seulement par Pierre-Paul Morin de Harel Drouin Gestion Conseil.

STRUCTURE DE DONNÉES INCLUSES:

Nom du client

adresse du client (no, rue, numéro bureau, ville, province, code postal) nom du contact: à qui est destinée la facture description du travail effectué

le montant de la facture

\section{VOLUME:}

Environ 25 par mois. 
Flux de données

\section{Copie blanche, 5.1.3-D5.1/7}

REF. SOURCE: 5.1.3

DESCRIPTION: Reproduire les factures

REF. DESTIN.: D5.1/7

DESCRIPTION: Dossier du client

\section{DESCRIPTION DÉTAILLÉE:}

Contient le nom du client, l'adresse, le nom du contact, le numéro du client, la description du travail effectué et le montant. Cette copie, qui va dans le dossier du client, est utilisé seulement par Jean-Guy Grenier de Harel Drouin Gestion Conseil.

STRUCTURE DE DONNÉES INCLUSES:

Nom du client

adresse du client (no, rue, numéro bureau, ville, province, code postal)

nom du contact: à qui est destinée la facture

description du travail effectué

le montant de la facture

VOLUME:

Environ 20 par mois. 
Flux de données

\section{Copies de couleur, 5.1.3-D5.1/1}

REF. SOURCE: 5.1 .3

DESCRIPTION: Reproduire les factures

REF. DESTIN.: D5.1/1

DESCRIPTION: Copies en couleur des factures

\section{DESCRIPTION DÉTAILLÉE:}

Contient le nom du client, l'adresse, le nom du contact, le numéro du client, la description du travail effectué et le montant.

. copies vertes: Harel, Drouin et associés

. copies roses: Harel Drouin Gestion Conseil, Pierre-Paul Morin

. copies bleues: Harel Drouin Gestion Conseil, Jean-Guy Grenier

. copies jaunes: Harel Drouin Gestion Conseil, Gérald Bélanger

STRUCTURE DE DONNÉES INCLUSES:

Nom du client

adresse du client (no, rue, numéro bureau, ville, province, code postal) nom du contact: à qui est destinée la facture description du travail effectué

le montant de la facture

VOLUME:

Environ 400 par mois. 
Flux de données

\section{Copie blanche, 5.1.3-D3}

REF. SOURCE: 5.1 .3

DESCRIPTION: Reproduire les factures

REF. DESTIN.: D3

DESCRIPTION: Dossier de correspondance

DESCRIPTION DÉTAILLÉE:

Contient le nom du client, l'adresse, le nom du contact, le numéro du client, la description du travail effectué et le montant.

STRUCTURE DE DONNÉES INCLUSES:

Nom du client

adresse du client (no, rue, numéro bureau, ville, province, code postal)

nom du contact: à qui est destinée la facture

description du travail effectué

le montant de la facture

VOLUME:

Environ $\mathbf{4 0 0}$ par mois. 
Flux de données

Factures, 5.1.3-a

REF. SOURCE: 5.1 .3

DESCRIPTION: Reproduire les factures

REF. DESTIN.: a

DESCRIPTION: Client

DESCRIPTION DÉTAILLÉE:

Contient le nom du client, l'adresse, le nom du contact, le numéro du client, la description du travail effectué et le montant.

STRUCTURE DE DONNÉES INCLUSES:

Nom du client

adresse du client (no, rue, numéro bureau, ville, province, code postal)

nom du contact: à qui est destinée la facture

description du travail effectué

le montant de la facture

VOLUME:

Environ 400 par mois. 
Flux de données

Factures, 5.1.2-5.1.3

REF. SOURCE: 5.1.2

DESCRIPTION: Dactylographier la facture

REF. DESTIN.: 5.1 .3

DESCRIPTION: Reproduire les factures

DESCRIPTION DÉTAILLÉE:

Contient le nom du client, l'adresse, le nom du contact, le numéro du client, la description du travail effectué et le montant.

STRUCTURE DE DONNÉES INCLUSES:

Nom du client

adresse du client (no, rue, numéro bureau, ville, province, code postal)

nom du contact: à qui est destinée la facture

description du travail effectué

le montant de la facture

\section{VOLUME:}

Environ 400 par mois. 
Flux de données

\section{Brouillon de factures, 5.1.1-5.1.2}

REF. SOURCE: $\mathbf{5 . 1 . 1}$

DESCRIPTION: Préparer la facture

REF. DESTIN.: 5.1 .2

DESCRIPTION: Dactylographier les factures

DESCRIPTION DÉTAILLÉE:

Copies de la facture antérieure sur laquelle on apporter les corrections afin de produire la facture courante.

STRUCTURE DE DONNÉES INCLUSES:

Nom du client

adresse du client (no, rue, numéro bureau, ville, province, code postal) nom du contact: à qui est destinée la facture description du travail effectué

le montant de la facture

VOLUME:

Maximum 400 par mois. 
Flux de données

\section{Travail effectué, b-5.1.1}

REF. SOURCE: $b$

REF. DESTIN.: 5.1.1
DESCRIPTION: Employés

DESCRIPTION: Préparer la facture

DESCRIPTION DÉTAILLÉE:

Cette information provient de l'employé qui, à partir de son agenda, informe le vérificateur senior ou l'associé sur le travail effectué dans le dossier.

STRUCTURE DE DONNÉES INCLUSES:

Description narrative du travail effectué.

VOLUME:

On ne peut quantifier cette information. 
Flux de données

\section{Nombre d'heures, b-5.1.1}

REF. SOURCE: $b$

REF. DESTIN.: 5.1.1
DESCRIPTION: Employés

DESCRIPTION: Préparer la facture

\section{DESCRIPTION DÉTAILLÉE:}

Nombre d'heures qu'un employé a travaillées sur le dossier d'un client depuis la dernière mise à jour des travaux en cours.

STRUCTURE DE DONNÉES INCLUSES:

Chiffires.

\section{VOLUME:}

1 chiffre par employé ayant travaillé sur le dossier. 
Flux de données

\section{Taux horaire, D5/6-5.1.1}

REF. SOURCE: D5/6

DESCRIPTION: Auxiliaire des travaux en cours

REF. DESTIN.: 5.1.1

DESCAIPTION: Préparer la facture

DESCRIPTION DÉTAILLÉE:

Taux horaire de chaque employé ayant travaillé sur le dossier du client.

STRUCTURE DE DONNÉES INCLUSES:

Chiffre.

VOLUME:

1 par employé. 
Flux de données

\section{Montant imputé, D5/6-5.1.1}

REF. SOURCE: D5/6

REF. DESTIN.: 5.1.1
DESCRIPTION: Auxiliaire des travaux en cours

DESCRIPTION: Préparer la facture

DESCRIPTION DÉTAILLÉE:

Le total du temps facturable et des déboursés imputés au client pour le mois.

STRUCTURE DE DONNÉES INCLUSES:

Chiffre.

VOLUME:

1 par client. 
Flux de données

\section{Copie facture antérieure, d3-5.1.1}

REF. SOURCE: D3

DESCRIPTION: Dossier de correspondance

REF. DESTIN.: 5.1.1

DESCRIPTION: Préparer la facture

DESCRIPTION DÉTAILLÉE:

La copie de la facture antérieure qui est brochée dans le dossier de correspondance est utilisée par les vérificateurs seniors et certains associés de Harel, Drouin et Associés comme brouillon de facture. Sur cette copie, ils effectuent les corrections nécessaires à la production de la nouvelle facture.

Dans le cas de Harel Drouin Gestion Conseil, il n'existe pas de copie antérieure car il s'agit toujours de nouveau client.

\section{STRUCTURE DE DONNÉES INCLUSES:}

Nom du client

adresse du client (no, rue, numéro bureau, ville, province, code postal)

nom du contact: à qui est destinée la facture

description du travail effectué

le montant de la facture

\section{VOLUME:}

1 par dossier. 


\section{Processus 5.2, Enregistrer les recettes}




\section{Diagrammes de flux de données}




\subsection{Enregistrer les recettes}

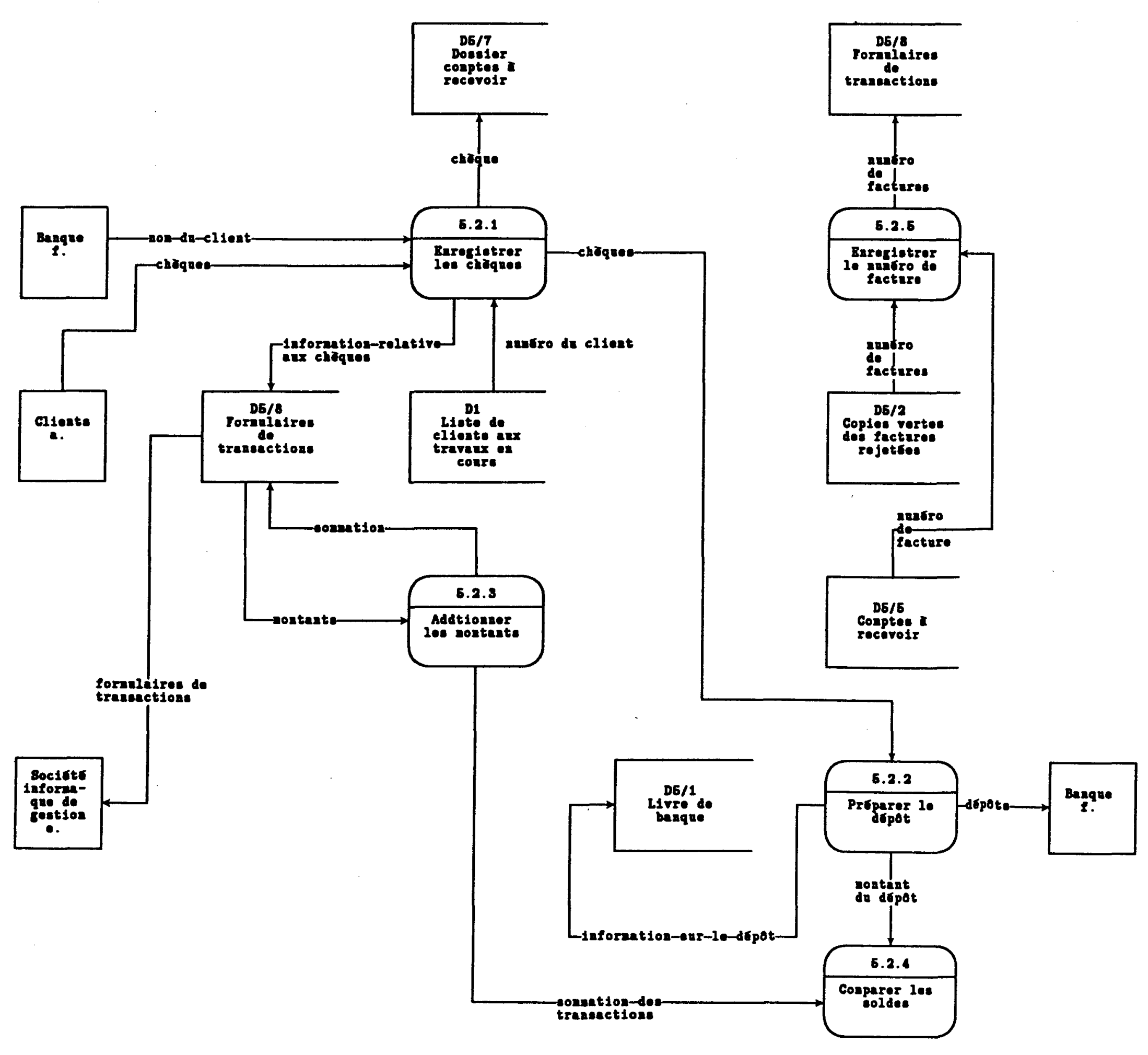




\section{Processus de traitement}


Processus de traitement

\section{Préparer le dépôt, 5.2.2}

\section{DESCRIPTION}

Consiste à inscrire sur le bordereau de dépôt, le montant du dépôt et à estamper la mention "pour dépót seulement".

ENTRÉES

Chèques, 5.2.1-5.2.2
SORTIE

Dépôt, 5.5.2-F

RÉSUMÉ DE LA LOGIQUE:

- Additionner tous les chèques reçus

- Inscrire le résultat de l'addition sur le bordereau de dépôt

- Inscrire la date du dépót sur le bordereau de dépôt 
Processus de traitement

\section{Enregistrer les chèques, 5.2.1}

\section{DESCRIPTION}

Consiste à reporter l'information relative aux chèques sur le formulaire de transactions afin d'effectuer la mise à jour des comptes à recevoir.

ENTRÉES

Nom du client, F-5.2.1

Chèques, a-5.2.1

Numéro du client, D1-5.2.1

Information relative aux chèques, D5/8-5.2.1

\section{SORTIE}

Information relative aux chèques, 5.2.1-D5/8

Chèque, 5.2.1-D5/7

Chèques, 5.2.1-5.2.2

RÉSUMÉ DE LA LOGIQUE:

- Si signature illisible - appeler à la banque émettrice pour connaître le nom du signataire

- Inscrire le nom du client dans la section description

- Inscrire le numéro du client

- Inscrire le montant du chèque 
Processus de traitement

\section{Additionner les montants, 5.2.3}

\section{DESCRIPTION}

Pour chaque formulaire de transactions prendre les montants qui y sont inscrits et les additionner. Inscrire le chiffre obtenu au bas du formulaire. Additionner avec une calculatrice à ruban tous les montants inscrits au bas de chaque formulaire de transactions. Joindre le ruban de la calculatrice aux formulaires de transactions assemblés.

\section{ENTRÉES}

Montants, D5/8-5.2.3

\section{SORTIE}

Sommation des transactions, 5.2.3-5.2.4

Sommation, 5.2.3-D5/8

\section{RÉSUMÉ DE LA LOGIQUE:}

- Additionner tous les montants inscrits sur un formulaire

- Inscrire le résultat de l'addition au bas du formulaire

- Additionner, avec une calculatrice à ruban, tous les montants inscrits au bas du formulaire

- Brocher le ruban de la calculatrice et les formulaires ensembles. 
Processus de traitement

\section{Comparer les soldes, 5.2.3}

\section{DESCRIPTION}

Comparer le montant du dépôt enregistré sur le bordereau de dépôt avec le montant obtenu en additionnant ensemble tous les formulaires de transactions.

\section{ENTRÉES}

Montants de dépôt, 5.2.2-5.2.4

Sommation des transactions, 5.2.3-5.2.4
SORTIE

Aucune

\section{RÉSUMÉ DE LA LOGIQUE:}

- Comparer le montant du dépôt et le total des montants inscrits au bas des formulaires de transactions

- Si différence vérifier le montant du dépôt et vérifier les montants inscrits au bas des formulaires de transactions. 
Processus de traitement

\section{Enregistrer le numéro de la facture, 5.2.5}

\section{DESCRIPTION}

II s'agit de retracer le numéro de la facture qui a été envoyée aux clients et de l'inscrire sur le formulaire de transactions.

\section{ENTRÉES}

Numéro de facture, D5/4, 5.2.5

Numéro de facture, $D 5 / 2-5.2 .5$

Numéro de facture, D5/5-5.2.5

Numéro de facture, D5/3-5.2.5
SORTIE

Numéro de facture, 5.2.5-D5/8

\section{RÉSUMÉ DE LA LOGIQUE:}

- Si facture de Harel, Drouin et Associés

- chercher, à l'aide de l'écran cathodique, dans les comptes à recevoir la facture correspondante au chèque reçu

- Si la facture n'apparaît pas

- si le nombre de factures rejetées est restreint

- regarder dans les factures rejetées

- sinon regarder dans le rapport nommé "Liste des comptes à recevoir, Harel, Drouin et Associés"

- Si facture de Harel Drouin et Gestion Conseil

- regarder dans le rapport nommé "Liste des comptes à recevoir Harel Drouin Gestion Conseil" 
Flux de données 
Flux de données

\section{Numéro du client, d1-5.2.1}

REF. SOURCE: D1

DESCRIPTION: Liste de clients travaux en cours

REF. DESTIN.: 5.2.1

DESCRIPTION: Enregistrer les chèques

DESCRIPTION DÉTAILLÉE:

Numéro du client qui est nécessaire pour la mise à jour des comptes à recevoir.

STRUCTURE DE DONNÉES INCLUSES:

Numéro

VOLUME:

300 par mois. 
Flux de données

\section{Information relative au chèque, 5.2.1-D5/8}

REF. SOURCE: 5.2.1

REF. DESTIN.: D5/8
DESCRIPTION: Enregistrer les chèques

DESCRIPTION: Formulaires de transactions

\section{DESCRIPTION DÉTAILLÉE:}

Le nom du client, son numéro, le montant du chèque nécessaire à la mise à jour des comptes à recevoir.

STRUCTURE DE DONNÉES INCLUSES:

Numéro de compte, numéro du sous-compte, montant du chèque, nom du client.

VOLUME:

300 par mois. 
Flux de données

\section{Chèques, 5.2.1-5.2.2}

REF. SOURCE: 5.2.1

DESCRIPTION: Enregistrer les chèques

REF. DESTIN.: 5.2 .2

DESCRIPTION: Préparer le dépôt

DESCRIPTION DÉTAILLÉE:

Contient l'information relative au paiement d'une facture.

STRUCTURE DE DONNÉES INCLUSES:

Date du chèque, nom du destinataire, montant du chèque en chiffre, montant du chèque en lettre ou inscrit à l'aide d'un protectographe, signature.

VOLUME:

300 par mois. 
Flux de données

\section{Nom du client, f-5.2.1}

REF. SOURCE: $F$

REF. DESTIN.: 5.2.1
DESCRIPTION: Banques

DESCRIPTION: enregistrer les chèques

\section{DESCRIPTION DÉTAILLÉE:}

Lorsque la signature du client n'est pas lisible et qu'il n'y a aucune référence sur le chèque, nous appelons à la banque pour connaître le nom de la personne dont le numéro de compte apparaît sur le chèque.

STRUCTURE DE DONNÉES INCLUSES:

Nom

VOLUME:

Quelques-uns par mois. 
Flux de données

Chèques, a-5.2.1

REF. SOURCE: a

DESCRIPTION: Clients

REF. DESTIN.: 5.2.1

DESCRIPTION: Enregistrer les chèques

DESCRIPTION DÉTAILLÉE:

Chèques envoyés par les clients pour payer nos honoraires.

STRUCTURE DE DONNÉES INCLUSES:

Date du chèque, nom du destinataire, montant du chèque en chiffre, montant du chèque en lettre ou inscrit à l'aide d'un protectographe, signature.

VOLUME:

300 par mois. 
Flux de données

\section{Chèques, 5.2.1-D5/7}

REF. SOURCE: 5.2.1

DESCRIPTION: Enregistrer les chèques

REF. DESTIN.: D5/7

DESCRIPTION: Dossier comptes à recevoir

\section{DESCRIPTION DÉTAILLÉE:}

Information relatives aux chèques envoyées par les clients. Ce flux est employé seulement par Jean-Guy Grenier et Gérald Bélanger de Harel Drouin Gestion Conseil.

STRUCTURE DE DONNÉES INCLUSES:

Date du chèque, nom du client ayant émis le chèque, montant du chèque.

VOLUME:

Maximum 20 par mois. 
Flux de données

\section{Chèques, 5.2.1-D5/7}

REF. SOURCE: 5.2.2

DESCRIPTION: Enregistrer les chèques

REF. DESTIN.: $\mathbf{5 . 2 . 4}$

DESCRIPTION: Dossier comptes à recevoir

\section{DESCRIPTION DÉTAILLÉE:}

Information relatives aux chèques envoyées par les clients. Ce flux est employé seulement par Jean-Guy Grenier et Gérald Bélanger de Harel Drouin Gestion Conseil.

STRUCTURE DE DONNÉES INCLUSES:

Date du chèque, nom du client ayant émis le chèque, montant du chèque.

VOLUME:

Maximum 20 par mois. 
Flux de données

\section{Montant du dépôt, 5.2.2-5.2.4}

REF. SOURCE: 5.2.2

REF. DESTIN.: F
DESCRIPTION: Préparer le dépôt

DESCRIPTION: Comparer les soldes

DESCRIPTION DÉTAILLÉE:

Montant qui provient de la sommation des chèques reçus.

STRUCTURE DE DONNÉES INCLUSES:

Chiffres

VOLUME:

20 par mois. 
Flux de données

Dépôts, 5.2.2-F

REF. SOURCE: $\mathbf{5 . 2 . 2}$

REF. DESTIN.: D5/1
DESCRIPTION: Préparer le dépôt

DESCRIPTION: Banques

\section{DESCRIPTION DÉTAILLÉE:}

Tous les chèques et le bordereaux de dépôt.

STRUCTURE DE DONNÉES INCLUSES:

Une centaine de chèques

Date du dépôt

Montant du dépôt

Signature du déposant

VOLUME:

8 dépôts par mois. 
Flux de données

\section{Information sur le dépôt, 5.2.2-D5/1}

REF. SOURCE: $\mathbf{5 . 2 . 2}$

REF. DESTIN.: D5/1
DESCRIPTION: Préparer le dépôt

DESCRIPTION: Livres de banque

DESCRIPTION DÉTAILLÉE:

Date et le montant du dépôt.

STRUCTURE DE DONNÉES INCLUSES:

Date

"Dépôt"

Montant du dépôt

VOLUME:

8 par mois. 
Flux de données

\section{Numéro de facture, 5.2.5-D5/8}

REF. SOURCE: $\mathbf{5 . 2 . 5}$

DESCRIPTION: Enregistrer le numéro de facture

REF. DESTIN.: D5/8

DESCRIPTION: Formulaire de transactions

DESCRIPTION DÉTAILLÉE:

Numéro qui est estampillé sur la copie de la facture par le département de la comptabilité.

STRUCTURE DE DONNÉES INCLUSES:

Numéro

VOLUME:

Environ 400 par mois. 
Flux de données

\section{Numéro de facture, D5/2-5.2.5}

REF. SOURCE: D5/2

REF. DESTIN.: 5.2 .5
DESCRIPTION: Copies vertes des factures rejetées

DESCRIPTION: Enregistrer numéro de facture

DESCRIPTION DÉTAILLÉE:

Numéro qui est estampillé sur la copie de la facture par le département de la comptabilité.

STRUCTURE DE DONNÉES INCLUSES:

Numéro

VOLUME:

400 par mois. 
Flux de données

\section{Numéro de facture, D5/5-5.2.5}

REF. SOURCE: D5/53

REF. DESTIN.: 5.2 .5
DESCRIPTION: Comptes à recevoir

DESCRIPTION: Enregistrer numéro de facture

\section{DESCRIPTION DÉTAILLÉE:}

Numéro qui est estampillé sur la copie de la facture par le département de la comptabilité.

STRUCTURE DE DONNÉES INCLUSES:

Numéro

VOLUME: 400 par mois. 
Flux de données

\section{Sommation, 5.2.3-D5/8}

REF. SOURCE: $\mathbf{5 . 2 . 3}$

DESCRIPTION: Additionner les montants

REF. DESTIN.: D5/8

DESCRIPTION: Formulaires de transactions

\section{DESCRIPTION DÉTAILLÉE:}

Le numéro du compte bancaire et l'addition de toutes les transactions enregistrées sur un formulaire de transactions. Le résultat de cette addition représente le montant du dépôt.

STRUCTURE DE DONNÉES INCLUSES:

Numéro du compte

Montant du dépôt

VOLUME:

1 par formulaire. 
Flux de données

\section{Formulaire de transactions, D5/8-e}

REF. SOURCE: D5/8

DESCRIPTION: Formulaire de transactions

REF. DESTIN.: $\theta$

DESCRIPTION: Société d'informatique de gestion

DESCRIPTION DÉTAILLÉE:

Formulaires qui contiennent les noms des clients, les montants des chèques, le numéro de compte dans lequel seront imputés les chèques, les numéros des sous-comptes et les numéros des factures payées. La dernière écriture du formulaire contient le numéro du compte bancaire dans lequel sera versé le dépôt et le montant du dépôts.

STRUCTURE DE DONNÉES INCLUSES:

Numéro de la facture

Numéro de compte

Numéro du sous-compte

Montant

Description

Total des montants inscrits

VOLUME:

8 par mois. 
Flux de données

\section{Sommation des transactions, 5.2.3}

REF. SOURCE: $\mathbf{5 . 2 . 3}$

REF. DESTIN.: 5.2.4
DESCRIPTION: Additionner les montants

DESCRIPTION: Comparer les soldes

\section{DESCRIPTION DÉTAILLÉE:}

La sommation de tous les chèques enregistrés sur les formulaires de transactions.

STRUCTURE DE DONNÉES INCLUSES:

Chiffres

VOLUME:

1 
Flux de données

\section{Montant, D5/8-5.2.3}

REF. SOURCE: D5/8

REF. DESTIN.: 5.2.3
DESCRIPTION: Formulaires de transactions

DESCRIPTION: Additionner les montants

\section{DESCRIPTION DÉTAILLÉE:}

Montant de chacun des chèques enregistrés sur les formulaires de transactions.

STRUCTURE DE DONNÉES INCLUSES:

Chiffre

VOLUME:

35 par formulaire. 
Processus 5.4, Concilier les comptes de banque 
110

Diagrammes de flux de données 


\subsection{Concilier les comptes de banque}

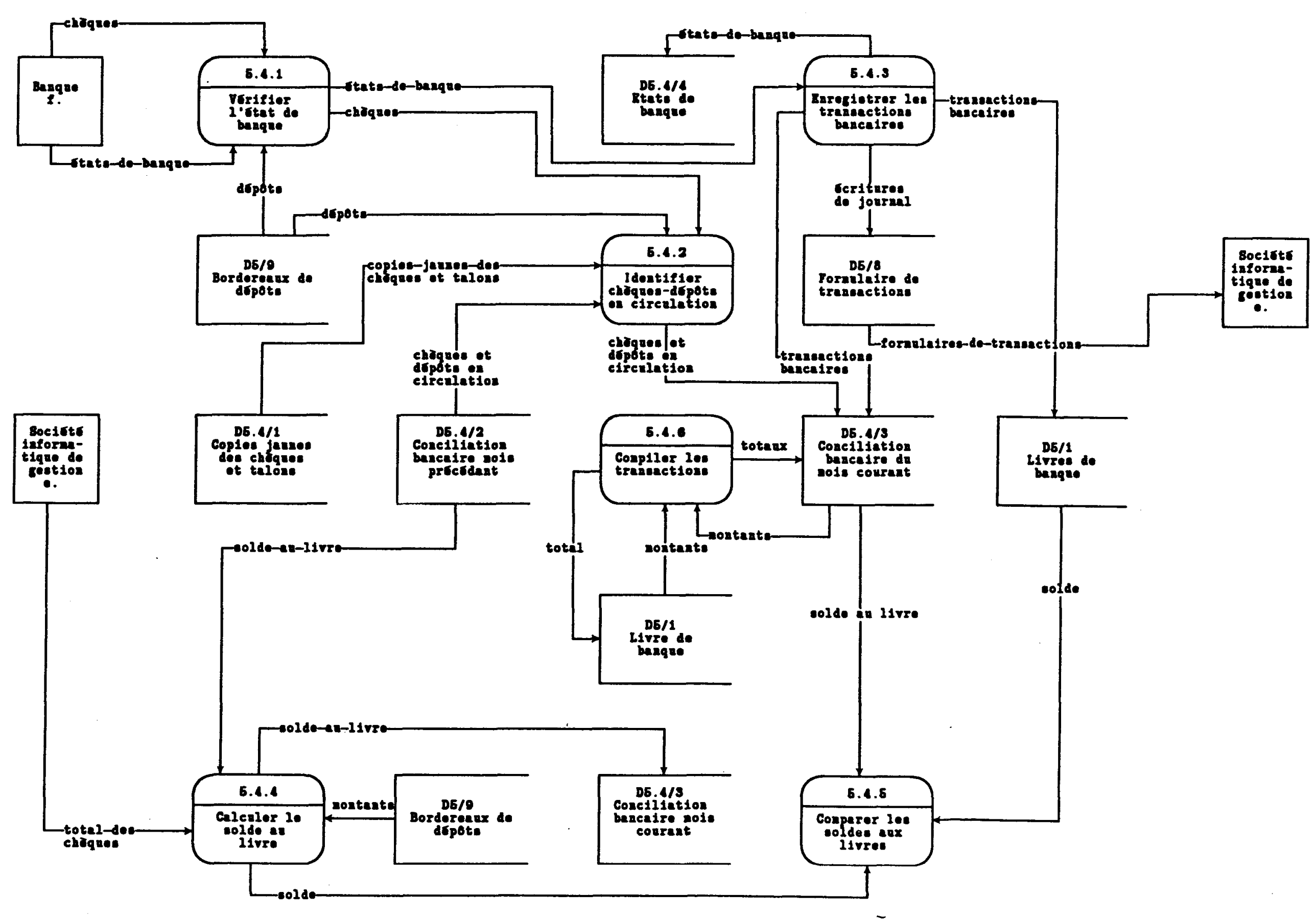


Processus de traitement 
Processus de traitement

\section{Vérifier l'état de banque, 5.4.1}

\section{DESCRIPTION}

Lorsqu'on vérifie l'état de banque, on s'assure que les chèques retournés par la banque soient enregistrés correctement sur l'état de banque. De plus, on s'assure que tous les montants débités au compte de banque figurent parmi les chèques reçus et, s'il ne s'agit pas de chèque, que les transactions inscrites soient reconnues par le département de comptabilité (intérêts, frais bancaires, etc). Le département de comptabilité vérifie cinq états de banque.

\section{ENTRÉES}

Chèques, f-5.4.1

États de banque, f-5.4.1

Dépôts, D5/9-5.4.1
SORTIE

Chèques, 5.4.1-5.4.2

États de banque, 5.4.1-5.4.3

\section{RÉSUMÉ DE LA LOGIQUE:}

- pour toutes les pièces reçues de la banque:

- prendre la pièce (généralement un chèque) et le pointer sur l'état de banque

- S'il y a des débits autre que des frais bancaires et paiements quelconque qui ne sont pas justifiés par une pièce (chèque, avis de débit, etc.) retournée par la banque, appeler la banque pour signaler l'erreur

- si le montant du chèque ou dépôt ne correspond pas à ce qui est inscrit sur l'état de banque appeler la banque pour signaler

l'erreur

- classer les chèques dans l'ordre numérique

- prendre les bordereaux de dépôts du mois et pointer sur le bordereau et sur l'état de banque les dépôts qui apparaissent sur l'état de banque 
Processus de traitement

\section{Identifier les chèques et dépôts} EN CIRCULATION

\section{DESCRIPTION}

Une fois que les chèques sont classés dans l'ordre numérique, chaque chèque est comparé à la liste de chèques apparaissant sur la conciliation bancaire du mois précédent ou aux copies jaunes des chèques. Tous les chèques apparaissant sur la conciliation bancaire du mois précédant pour lesquels nous n'avons pas le chèque, toutes les copies jaunes pour lesquels nous n'avons pas le chèque sont considérées comme étant des chèques en circulation.

\section{ENTRÉES}

Chèques, 5.4.1-5.4.2
SORTIE

Chèques et dépôts en circulation,

5.4.2-D5.4/3

Copie jaune chèque et talon,

$$
\text { 5.4/1-5.4.2 }
$$

Chèques et dépôt en circulation,

$$
\text { D5.4/2-5.4.2 }
$$

Dépôts, D5/9-5.4.2

\section{RÉSUMÉ DE LA LOGIQUE:}

- reporter sur la conciliation bancaire du mois courant, tous les chèques qui apparaissent sur la conciliation bancaire du mois précédent pour lesquels nous n'avons pas le chèque

- reporter, sur la conciliation bancaire courante, l'information contenue sur toutes les copies jaunes pour lesquelles nous n'avons pas reçu le chèque

- reporter sur la conciliation bancaire du mois tous les dépôts du mois qui n'ont pas été pointés lors de l'opération "vérifier l'état de banque" 
Processus de traitement

\section{Enregistrer les transactions \\ BANCAIRES, 5.4.3}

\section{DESCRIPTION}

L'opération "Enregistrer les transactions bancaires" consiste à inscrire sur la conciliation bancaire, sur un formulaire de transaction et dans le livre servant au suivi bancaire, toutes les transactions qui ont modifié le solde bancaire et qui n'ont pas été enregistrées dans les livres comptables (ex: frais de banque, intérêts etc.)

ENTRÉES

État de banque, 5.4.1-5.4.3
SORTIE

États de banque, 5.4.3-D5.5/4

Écritures de journal, 5.4.3-D5/8

Transactions bancaires, 5.4.3-D5/1

\section{RÉSUMÉ DE LA LOGIQUE:}

- prendre l'état de banque et enregistrer toutes les transactions qui ne sont pas pointées:

- dans le livre de banque

- sur un formulaire de transactions

- sur la conciliation bancaire du mois courant 
Processus de traitement

\section{Calculer le solde au livre, 5.4.4}

\section{DESCRIPTION}

On calcule le solde qui devrait être inscrit dans le livre de banque en additionnant au "solde aux livres" apparaissant sur la conciliation bancaire du mois précédant les dépôts du mois et en y soustrayant les chèques. Le montant ainsi obtenu est inscrit sur la conciliation du mois courant.

\section{ENTRÉES}

Total des chèques, e-5.4.4

Montants, D5/9-5.4.5

Solde au livre, D5.4/2-5.4.4

\section{RÉSUMÉ DE LA LOGIQUE:}

- prendre le solde au livre sur la conciliation bancaire du mois précédant

- y additionner tous les dépôts effectués durant le mois

- y soustraire le total des chèques effectués durant le mois

- reporter le montant ainsi obtenu sur la conciliation bancaire du mois courant
SORTIE

Solde au livre, 5.5.4-D5.4/3

Solde, 5.4.4-5.4.5 
Processus de traitement

\section{Comparer les soldes aux livres, 5.4.5}

\section{DESCRIPTION}

Suite au processus "comparer les soldes aux livres" le solde obtenu est comparé au solde inscrit dans le livre de banque. Advenant que le solde obtenu lors de l'opération "calculer le solde au livre" est différent du solde inscrit au livre de banque, des recherches sont entreprises afin d'identifier l'endroit où s'est glissée l'erreur et pour y apporter les corrections.

ENTRÉES

SORTIE

Solde, 5.4.4-5.4.5.

Solde D5/1-5.4.5

RÉSUMÉ DE LA LOGIQUE:

Si solde calculé à l'opération 5.4.4 égale solde au livre de banque

- rien faire

- sinon chercher l'erreur et la corriger 
Processus de traitement

\section{Compiler les transactions enregistrées, 5.4.6}

\section{DESCRIPTION}

Pour chacune des conciliations bancaires et chaque "livre de banque", nous compilons les transactions inscrites afin d'ajuster le "solde au livre" et le "solde de la banque" et de mettre à jour le solde du "livre de banque".

ENTRÉES

Montants, D5.4/3-5.4.6

Montants, D5/1-5.4.6
SORTIE

Totaux, 5.4.6-D5.4/3

Total, 5.4.6-D5/1

\section{RÉSUMÉ DE LA LOGIQUE:}

- $\quad$ prendre le "livre de banque" y ajouter tous les débits, y enlever tous les crédits, y inscrire le résultat obtenu

- prendre la conciliation bancaire

au solde de banque:

- ajouter les dépôts en circulation

- enlever tous les chèques en circulation

- inscrire le nouveau solde de banque obtenu

au solde du livre

- ajouter tous les intérêts créditeurs

- enlever tous les intérêts débiteurs

- enlever les frais de banque

- enlever tous les autres débiteurs

inscrire le nouveau solde au livre obtenu 
Flux de données 
Flux de données

Chèques, 5.4.1-5.4.2

REF. SOURCE: 5.4.1

REF. DESTIN.: 5.4 .2
Vérifier l'état de banque

Identifier les chèques en circulation

\section{DESCRIPTION DÉTAILLÉE:}

Chèques produits par:

Harel, Drouin et Associés

Harel Drouin Gestion Conseil

Harel Drouin et compagnie

Harel Drouin in trust

Les Services Harel Drouin

STRUCTURE DE DONNÉES INCLUSES:

Date du chèque

Nom du destinataire

Montant du chèque dactylographié

Montant du chèque inscrit à l'aide d'un protectographe

Signatures.

VOLUME: 400 par mois. 
Flux de données

\section{États de banque, f-5.4.1}

REF. SOURCE: $f$

REF. DESTIN.: 5.4.1
Banque

Vérifier l'état de banque

\section{DESCRIPTION DÉTAILLÉE:}

Contient toutes les transactions ayant affecté un compte de banque. Cinq états de banque parviennent au bureau de Harel Drouin: Harel, Drouin et Associés, Harel Drouin Gestion Conseil, Harel Drouin et cie, Les Services Harel Drouin et Harel Drouin in trust.

STRUCTURE DE DONNÉES INCLUSES:

Identification de la banque

Identification du client

Numéro de compte

Numéro de page

Date à laquelle la période se termine

Nombre d'effets

Type de compte

Date début période

Montant au début de la période

Pour chacune des transactions:

- description de la transaction

- montant de la transaction

. date de la transaction

solde à la fin

VOLUME:

5 par mois. 
Flux de données

Chèques, f-5.4.1

REF. SOURCE: $f$

REF. DESTIN.: 5.4.1
Banque

Vérifier l'état de banque

\section{DESCRIPTION DÉTAILLÉE:}

Chèques produits par:

Harel, Drouin et associés

Harel Drouin Gestion Conseil

Harel Drouin et cie

Harel Drouin in trust

Les Services Harel Drouin

STRUCTURE DE DONNÉES INCLUSES:

Date du chèque

Nom du destinataire

Montant du chèque dactylographié

Montant du chèque inscrit à l'aide d'un protectographe

Signatures.

VOLUME:

400 par mois. 
Flux de données

\section{États de banque, 5.4.1-5.4.3}

REF. SOURCE: 5.4.1

Vérifier l'état de banque

REF. DESTIN.: 5.4.3

Enregistrer les transactions bancaires

DESCRIPTION DÉTAILLÉE:

Contient toutes les transactions ayant affecté un compte de banque.

STRUCTURE DE DONNÉES INCLUSES:

Identification de la banque

Identification du client

Numéro de compte

Numéro de page

Date à laquelle la période se termine

Nombre d'effets

Type de compte

Date début période

Montant au début de la période

Pour chacune des transactions:

- description de la transaction

- montant de la transaction

- date de la transaction

solde à la fin

VOLUME:

5 par mois. 
Flux de données

\section{États de banque, 5.4.3, D5.4/4}

REF. SOURCE: $\mathbf{5 . 4 . 3}$

REF. DESTIN.: D5.4/4
Enregistrer les transactions bancaires

États de banque

DESCRIPTION DÉTAILLÉE:

Contient toutes les transactions ayant affecté un compte de banque.

STRUCTURE DE DONNÉES INCLUSES:

Identification de la banque

Identification du client

Numéro de compte

Numéro de page

Date à laquelle la période se termine

Nombre d'effets

Type de compte

Date début période

Montant au début de la période

Pour chacune des transactions:

- description de la transaction

- montant de la transaction

- date de la transaction

solde à la fin

VOLUME: 5 par mois. 
Flux de données

\section{Écritures de journal, 5.4.3-D5/8}

REF. SOURCE: $\mathbf{5 . 4 . 3}$

REF. DESTIN.: D5/8
Enregistrer les transactions bancaires

Formulaires de transactions

\section{DESCRIPTION DÉTAILLÉE:}

Représentent toutes les transactions passées dans les comptes de banques (ex.: frais de banque, intérêts) qui n'ont pas été enregistrées dans les livres. Les Services Harel Drouin accuse le plus grand nombre de transactions.

STRUCTURE DE DONNÉES INCLUSES:

Jour de la transaction

Référence: régularisation

Numéro de compte

Numéro du sous-compte

Montant

Description

VOLUME:

Maximum environ 40 par mois pour chaque compte. 
Flux de données

\title{
Montants, D5/1-5.4.6
}

\author{
REF. SOURCE: D5/1 \\ Livres de banque \\ REF. DESTIN.: 5.4.6 \\ Additionner les transactions enregistrées
}

\section{DESCRIPTION DÉTAILLÉE:}

Le montant de chaque transaction qui a été enregistrée dans le livre de banque.

STRUCTURE DE DONNÉES INCLUSES:

Chiffres.

VOLUME:

Maximum 40 par compte de banque. 
Flux de données

\section{Total, 5.4.6-D5/1}

REF. SOURCE: 5.4 .6

REF. DESTIN.: D5/1
Additionner les transactions enregistrées

Livres de banque

\section{DESCRIPTION DÉTAILLÉE:}

Résultat provenant de l'addition ou de la soustraction du montant d'une transaction bancaire au solde précédant.

STRUCTURE DE DONNÉES INCLUSES:

Chiffres.

VOLUME:

Maximum 40 par compte de banque. 
Flux de données

\section{Transactions bancaires, 5.4.3-D5/1}

REF. SOURCE: 5.4 .3

Enregistrer les transactions bancaires

REF. DESTIN.: D5/1

Livres de banque

\section{DESCRIPTION DÉTAILLÉE:}

Débit ou un crédit ayant affecté le solde de banque.

STRUCTURE DE DONNÉES INCLUSES:

Date de la transaction

Nom de la transaction

Montant de la transaction (placé pour la colonne débit ou crédit selon le cas)

Solde du compte de banque.

\section{VOLUME:}

Maximum 40 par compte. 
Flux de données

\section{Transactions bancaires, 5.4.3-D5.4/3}

REF. SOURCE: $\mathbf{5 . 4 . 3}$

REF. DESTIN.: D5.4/3
Enregistrer les transactions bancaires

Conciliation bancaire mois courant

\section{DESCRIPTION DÉTAILLÉE:}

Débits ou crédits ayant affecté la banque. Ces transactions sont inscrites sur la conciliation bancaire afin de modifier le solde de banque qui est enregistré au livre.

STRUCTURE DE DONNÉES INCLUSES:

Chiffres

VOLUME:

Maximum 40 par compte. 
Flux de données

\section{Copie jaune chèque et talon, D5.4/1-5.4.2}

REF. SOURCE: D5.4/1

REF. DESTIN.: 5.4 .2
Copie jaune chèque et talon

Identifier chèques en circulation

DESCRIPTION DÉTAILLÉE:

Copie du chèque et du talon.

STRUCTURE DE DONNÉES INCLUSES:

Numéro du chèque

Nom de l'émetteur

Adresse de l'émetteur

Montant du chèque inscrit à l'aide du protectographe

Montant du chèque

Date du chèque

Nom du destinataire

Mention non négociable

Nom de la banque

Adresse de la banque

Date du chèque

Code(s) d'imputation

Montant(s) imputé(s)

Nom du fournisseur

Information "déjà chargé" ou "à charger"

VOLUME:

400 par mois. 
Flux de données

\section{Chèques et dépôts en circulation, D5.4/2-5.4.2}

REF. SOURCE: D5.4/2

REF. DESTIN.: $\mathbf{5 . 4 . 2}$
Conciliation bancaire mois précédant

Identifier chèques en circulation

DESCRIPTION DÉTAILLÉE:

Tous les chèques et les dépôts qui apparaissaient sur la conciliation bancaire du mois précédant et qui n'ont pas encore figurés sur l'état de banque.

STRUCTURE DE DONNÉES INCLUSES:

Chèques en circulation, pour chaque chèque:

date du chèque

numéro du chèque

nom du destinataire

Montant pour les dépôts en circulation:

date du dépôt

montant du dépôt

VOLUME: 
Flux de données

\section{Chèques et dépôts en circulation, 5.4.2-D5.4/3}

REF. SOURCE: 5.4 .2

REF. DESTIN.: D5.4/3
Chèques en circulation

Conciliation bancaire mois courant

\section{DESCRIPTION DÉTAILLÉE:}

Tous les chèques et les dépôts qui ont été effectuées mais qui ne figurent pas à l'état de banque.

STRUCTURE DE DONNÉES INCLUSES:

Chèques en circulation, pour chaque chèque:

date du chèque

numéro du chèque

nom du destinataire

montant

dépôt en circulation

date du dépôt

montant du dépôt

VOLUME: 
Flux de données

\section{Dépôts, D5/9-5.4.2}

REF. SOURCE: D5/9

REF. DESTIN.: 5.4 .2
Bordereaux de dépôts

Identifier chèques et dépôts en circulation

\section{DESCRIPTION DÉTAILLÉE:}

Vérifier si les montants des dépôts inscrits sur l'état de banque correspondent au montant des dépôts effectués.

STRUCTURE DE DONNÉES INCLUSES:

Chiffres.

VOLUME:

Environ 20 par mois. 
Flux de données

\section{Montant, D5.4/3-5.4.6}

REF. SOURCE: D5.4/3

REF. DESTIN.: 5.4 .6
Conciliation bancaire mois courant

Additionner les transactions enregistrées

\section{DESCRIPTION DÉTAILLÉE:}

Les chiffres de chaque transaction enregistrée sur la conciliation bancaire du mois courant.

STRUCTURE DE DONNÉES INCLUSES:

Chiffres.

VOLUME: Maximum 40 par conciliation. 
Flux de données

\section{Totaux, 5.4.6-D5.4/3}

REF. SOURCE: 5.4 .6

REF. DESTIN.: D5.4/3
Additionner les transactions enregistrées

Conciliation bancaire mois courant

\section{DESCRIPTION DÉTAILLÉE:}

Total des débits qui affectent le solde du livre, total des crédits qui affectent le solde au livre, nouveau solde au livre.

Total des débits qui affectent le solde bancaire, total des crédits qui affectent le solde bancaire, nouveau solde bancaire.

STRUCTURE DE DONNÉES INCLUSES:

Chiffres.

VOLUME:

6 par conciliation. 
Flux de données

\section{Solde au livre, D5.4/2-5.4.4}

REF. SOURCE: D5.4/2

REF. DESTIN.: 5.4 .4
Conciliation bancaire mois précédant

Calculer le solde au livre

\section{DESCRIPTION DÉTAILLÉE:}

C'est le solde au livre calculé à partir de la conciliation bancaire du mois précédant.

STRUCTURE DE DONNÉES INCLUSES:

Chiffre.

VOLUME:

1 par conciliation. 
Flux de données

\section{Total des chèques, e-5.4.4}

REF. SOURCE: $\theta$

REF. DESTIN.: 5.4.4
Société d'informatique de gestion

Calculer le solde au livre

\section{DESCRIPTION DÉTAILLÉE:}

Total qui représente la sommation des chèques produits au cours du mois.

STRUCTURE DE DONNÉES INCLUSES:

Chiffre.

VOLUME:

5 par mois. 
Flux de données

Montants, D5/9-5.4.4

REF. SOURCE: D5/9

REF. DESTIN.: 5.4.4
Bordereaux de dépôt

Calculer le solde au livre

DESCRIPTION DÉTAILLÉE:

Montants de tous les dépôts effectués durant le mois.

STRUCTURE DE DONNÉES INCLUSES:

Chiffres.

VOLUME:

25 par mois. 
Flux de données

\section{Solde au livre, 5.4.4-D5.4/3}

REF. SOURCE: 5.4 .4

Calculer le solde au livre

REF. DESTIN.: D5.4/3

Conciliation bancaire mois courant

DESCRIPTION DÉTAILLÉE:

Solde obtenu à partir du solde de la conciliation bancaire du mois précédant auquel nous avons ajouter tous les dépôts effectués au cours du mois et enlever tous les chèques produits durant le mois.

STRUCTURE DE DONNÉES INCLUSES:

Chiffre.

VOLUME:

5 par mois. 
Flux de données

Solde, 5.4.4-5.4.5

REF. SOURCE: $\mathbf{5 . 4 . 4}$

Calculer le solde au livre

REF. DESTIN.: 5.4 .5

Comparer les soldes aux livres

\section{DESCRIPTION DÉTAILLÉE:}

Solde obtenu à partir du solde de la conciliation bancaire du mois précédant auquel nous avons ajouter tous les dépóts effectués au cours du mois et enlever tous les chèques produits durant le mois.

STRUCTURE DE DONNÉES INCLUSES:

Chiffre.

VOLUME:

5 par mois. 
Flux de données

Solde, D5/1-5.4.5

REF. SOURCE: D5/1

REF. DESTIN.: 5.4.5
Livres de banque

Comparer les soldes aux livres

DESCRIPTION DÉTAILLÉE:

Montant inscrit sous la colonne "solde" que l'on retrouve dans "le livre de banque".

STRUCTURE DE DONNÉES INCLUSES:

Chiffre.

VOLUME:

5 
Flux de données

\section{Formulaires de transactions, D5/8-e}

REF. SOURCE: D5/8

REF. DESTIN.: e
Formulaires de transactions

Société d'informatique de gestion

DESCRIPTION DÉTAILLÉE:

Formulaires qui contiennent toutes les informations nécessaires à la mise à jour des livres comptables.

STRUCTURE DE DONNÉES INCLUSES:

page

nom du client

période

numéro du client

numéro du journal

nom du journal

mois

année

pour chacune des transactions:

jour de la transactions

référence: régularisation

numéro de compte

numéro du sous-compte

montant

description

VOLUME:

Maximum 6 par mois. 
Flux de données

\section{Copies jaunes des chèques et talon, e-D5.4/1}

REF. SOURCE: e

REF. DESTIN.: D5.4/1
Société d'informatique de gestion

Copies jaunes des chèques et talon

\section{DESCRIPTION DÉTAILLÉE:}

Copie du chèque et talon.

STRUCTURE DE DONNÉES INCLUSES:

Partie du chèque:

- numéro du chèque

- nom de l'émetteur

- adresse de l'émetteur

- montant du chèque inscrit à l'aide du protectographe

- montant du chèque

- date du chèque

- nom du destinataire

- mention non négociable

- nom de la banque

- adresse de la banque

Partie talon:

- date du chèque

- code(s) d'imputation

- montant(s) imputé(s)

- nom du fournisseur

- information si déjà chargé ou à charger

VOLUME:

400 par mois. 
Processus 5.5, Payer les factures des fournisseurs 


\subsection{Payer les factures des fournisseurs}

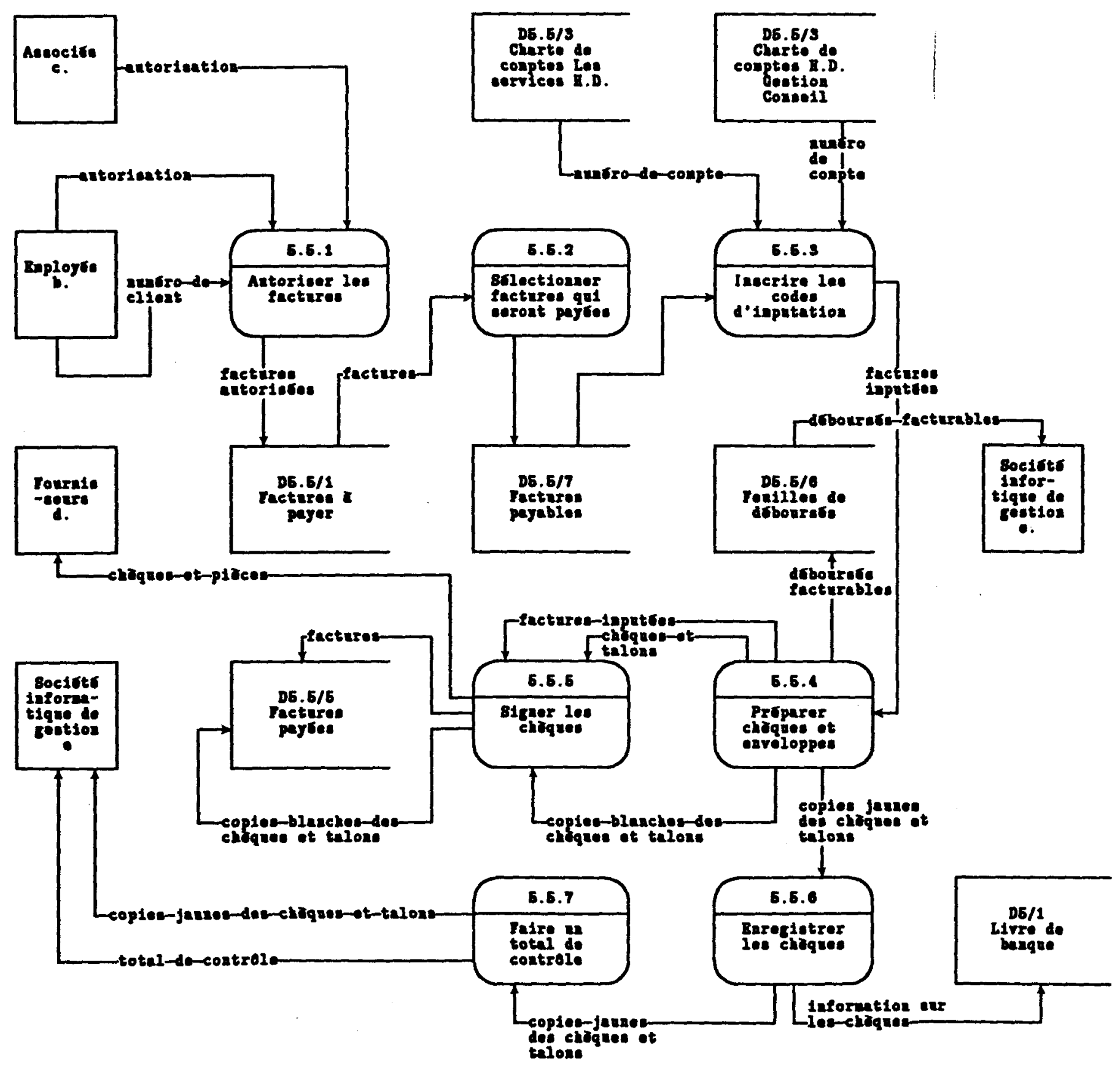




\section{Processus de traitement}


Processus de traitement

\section{Autoriser les factures, 5.5.1}

\section{DESCRIPTION}

L'autorisation d'une facture consiste à la parapher. Dans la majorité des cas les factures sont autorisées par les associés. Certains employés peuvent également autoriser des factures concernant l'achat de fournitures. Certaines factures autorisées sont accompagnées d'un numéro de client.

ENTRÉES

Autorisation, c-5.5.1

Numéro de client, c-5.5.1

Autorisation, b-5.5.1

RÉSUMÉ DE LA LOGIQUE:

- Regarder la facture

si c'est une location ne pas demander d'autorisation

si c'est un achat demander l'autorisation à l'associé concerné
SORTIE

Factures autorisées, 5.5.1-

D5.5/1 
Processus de traitement

Sélectionner les factures qui seront payées, 5.5.2

\section{DESCRIPTION}

Prendre la chemise de factures à payer et en retirer toutes les factures qui doivent être payées.

ENTRÉES

Factures, D5.5/1
SORTIE

Factures payables, 5.5.2-5.5.3

RÉSUMÉ DE LA LOGIQUE

Saisir la facture, regarder la date de la facture si la facture date de 30 jours mettre la facture de côté sinon la laisser dans la chemise 
Processus de traitement

\section{Inscrire les codes d'imputation 5.5.3}

\section{DESCRIPTION}

Prendre chacune des factures payables et y indiquer les codes d'imputation. S'il y a plusieurs factures pour le même fournisseur, les factures sont additionnées et le code d'imputation est inscrit sur le ruban de l'addition.

\section{ENTRÉES}

Factures payables, D5.5/7-5.5.3
SORTIE

Factures imputées, 5.5.4

RÉSUMÉ DE LA LOGIQUE:

Si facture de Harel Drouin et Associés

regarder dans la charte de compte correspondante le numéro de compte dans lequel sera imputé la facture

Si facture de Harel Drouin Gestion Conseil regarder dans la charte de compte correspondante le numéro du compte dans lequel sera imputé la facture

Si facture de Service Harel Drouin

regarder dans la charte de compte correspondante le numéro de compte dans lequel sera imputé la facture 
Processus de traitement

\section{Préparer les chèques et enveloppes, 5.5.4}

\section{DESCRIPTION}

Prendre chaque facture ou groupe de factures et faire le chèque qui paiera la ou les factures. Sur le talon du chèque, inscrire la date du chèque, la code d'imputation, le montant du chèque et le nom du fournisseur. Si la facture doit être facturée à un client, celle-ci est enregistrée sur une "formule de transactions".

ENTRÉES

Factures imputées, 5.5.3-5.5.4
SORTIE

Factures, 5.5.4-5.5.5

Chèques et talon, 5.5.4-5.5.5

Copies blanches des chèques et talon, 5.5.4-5.5.5

Déboursés facturables, 5.5.4-D5.5/6 Copies jaunes des chèques et talon 5.5.5-5.5.6

RÉSUMÉ DE LA LOGIQUE:

Inscrire sur le chèque:

la date du chèque

le montant du chèque

le nom du destinataire

le montant du chèque avec la protectographe.

Inscrire sur le talon du chèque:

la date du chèque

le(s) code(s) d'imputation

le(s) montant(s)

le nom du fournisseur

Si il y a un numéro de client sur la facture, inscrire sur une formule de transaction:

le numéro du client

le montant de la facture

le nom du fournisseur

Inscrire le nom du bénéficiaire et l'adresse sur une enveloppe. 
Processus de traitement

\section{Signer les chèques, $\mathbf{5 . 5 . 5}$}

\section{DESCRIPTION}

Les chèques sont signés par deux personnes: le directeur administratif (Réal Brière) et le directeur du bureau (Gilles Drouin). Le directeur administratif vérifie l'autorisation des factures, les codes d'imputation, le nom du bénéficiaire et la date du chèque.

ENTRÉES

Factures, 5.5.4-5.5.5

Chèques et talon, 5.5.4-5.5.5

Copies blanches des chèques et talon, 5.4.4-5.5.5
SORTIE

Chèques et talon, 5.5.5-d

Factures, 5.5.5-D5.5/5

Copies blanches chèques et talon, 5.5.5-D5.5/5

\section{RÉSUMÉ DE LA LOGIQUE:}

- $\quad$ regarder si la personne qui a autorisé la facture l'est, sinon demander l'autorisation

- regarder si code(s) d'imputation est (sont) exact(s) sinon modifier code(s) d'imputation

- vérifier si le nom du bénéficiaire est exact, sinon annuler le chèque, faire un autre chèque

- vérifier la date du chèque, si date inexacte, annuler le chèque, faire un autre chèque. 
Processus de traitement

\section{Enregistrer les chèques, 5.5.6}

\section{DESCRIPTION}

Prendre chaque copie jaune des chèques et les enregistrer un à un dans le livre de banque dans la colonne crédit.

\section{ENTRÉES}

Copies jaunes des chèques et talon, 5.5.4-5.5.6

\section{SORTIE}

Copies jaunes des chèques et talon, 5.5.6-5.5.7

Chèques et talon, 5.5.4-5.5.6

RÉSUMÉ DE LA LOGIQUE:

- inscrire la date du chèque

- inscrire le nom du bénéficiaire

- inscrire le montant du chèque

- calculer et inscrire le solde en banque 
Processus de traitement

\section{Faire un total de contrôle, 5.5.7}

\section{DESCRIPTION}

Prendre toutes les copies jaunes des chèques et additionner, sur une calculatrice à ruban, les montants qui y sont inscrits. Le montant ainsi obtenu est joint aux copies jaunes et est utilisé par les "K-punch" de la Société d'informatique de gestion pour vérifier l'entrée des données.

ENTRÉES

Copies jaunes des chèques et talon, 5.5.6-5.5.7

RÉSUMÉ DE LA LOGIQUE:

- Additionner le montant du chèque
SORTIE

Copies jaunes des chèques et talon, 5.5.7-e

Total de contrôle, 5.5.7-e 
Flux de données 
Flux de données

\section{Autorisation, c-5.5.1}

REF. SOURCE: $C$

REF. DESTIN.: 5.5.1
DESCRIPTION: Associés

DESCRIPTION: Autoriser les factures

DESCRIPTION DÉTAILLÉE:

L'autorisation consiste simplement à parapher une facture par un associé.

STRUCTURE DE DONNÉES INCLUSES:

Signature ou initiales d'un associé.

VOLUME:

400 par mois. 
Flux de données

\section{Numéro du client, c-5.5.1}

REF. SOURCE: $C$

REF. DESTIN.: 5.5.1
DESCRIPTION: Associés

DESCRIPTION: Autoriser les factures

DESCRIPTION DÉTAILLÉE:

Quelque fois, lorsqu'une facture est reliée au traitement d'un dossier d'un client, cette dernière sera facturée au client. Dans ce cas, l'associé concerné indique le numéro du client sur la facture.

STRUCTURE DE DONNÉES INCLUSES:

Numéro du client: 8 chiffres.

VOLUME:

Environ 200. 
Flux de données

\section{Autorisation, b-5.5.1}

REF. SOURCE: $b$

DESCRIPTION: Employés

REF. DESTIN.: 5.5.1

DESCRIPTION: Autoriser les factures

DESCRIPTION DÉTAILLÉE:

L'autorisation consiste simplement à parapher une facture par un employé dûment autorisé. Ces employés peuvent autoriser que des factures concernant des fournitures.

STRUCTURE DE DONNÉES INCLUSES:

Signature ou initiales.

VOLUME:

Environ 400 par mois. 
Flux de données

\section{Factures autorisées, 5.5.1-D5.5/1}

REF. SOURCE: $\mathbf{5 . 5 . 1}$

REF. DESTIN.: D5.5/1
DESCRIPTION: Autoriser les factures

DESCRIPTION: Factures à payer

DESCRIPTION DÉTAILLÉE:

Factures paraphées par un associé ou un employé selon le cas.

STRUCTURE DE DONNÉES INCLUSES:

Nom du fournisseur, date de la facture, description de l'achat, quantité, prix, unitaire, montant global, taxe de vente, total de la facture, autorisation.

VOLUME:

Environ 400 par mois. 
Flux de données

\section{Factures, D5.5/1-5.5.2}

REF. SOURCE: $5.5 / 1$

REF. DESTIN.: $\mathbf{5 . 5 . 2}$
DESCRIPTION: Factures à payer

DESCRIPTION: Sélectionner les factures qui seront payées

\section{DESCRIPTION DÉTAILLÉE:}

Contient l'ensemble des factures qui seront payées. Toutes ces factures doivent être autorisées sauf celles concernant la location de certains équipements ou représentant des mensualités.

STRUCTURE DE DONNÉES INCLUSES:

Nom du fournisseurs

Description des items achetés

Quantité achetées

Total de la facture

Autorisation.

VOLUME:

Entre 400 et 600 par mois. 
Flux de données

\section{Numéro de compte, D5.5/3-5.5.3}

REF. SOURCE: D5.5/3

REF. DESTIN.: 5.5 .3
DESCRIPTION: Charte de comptes Les Service Harel Drouin

DESCRIPTION: Inscrire les codes d'imputation

\section{DESCRIPTION DÉTAILLÉE:}

Numéro provenant de la charte de comptes de Les Services Harel Drouin.

STRUCTURE DE DONNÉES INCLUSES:

Chiffres.

VOLUME:

Entre 400 et 600 par mois. 
Flux de données

\section{Numéro de compte, D5.5/4-5.5.3}

REF. SOURCE: D5.5/4

REF. DESTIN.: 5.5.3 DESCRIPTION: Charte de comptes Harel, Drouin Gestion

DESCRIPTION: Inscrire les codes d'imputation

DESCRIPTION DÉTAILLÉE:

Numéro provenant de la charte de comptes de Harel Drouin Gestion Conseil.

STRUCTURE DE DONNÉES INCLUSES:

Numéro.

VOLUME:

Environ 50 par mois. 
Flux de données

Factures imputées, 5.5.3-5.5.4

REF. SOURCE: $\mathbf{5 . 5 . 3}$

DESCRIPTION: Inscrire les codes d'imputation

REF. DESTIN.: $\mathbf{5 . 5 . 4}$

DESCRIPTION: Préparer chèques et enveloppes

DESCRIPTION DÉTAILLÉE:

Factures contenant un ou plusieurs codes d'imputation.

STRUCTURE DE DONNÉES INCLUSES:

Nom du fournisseurs

Description des items achetés

Quantité des items achetés

Total de la facture

Autorisation

Code(s) d'imputation

Montant(s) à imputer.

VOLUME:

Environ 400 par mois. 
Flux de données

\section{Déboursés facturables, 5.5.4-D5.5/6}

REF. SOURCE: 5.5 .4

REF. DESTIN.: D5.5/6
DESCRIPTION: Préparer chèques et enveloppes

DESCRIPTION: Formulaire de transaction

\section{DESCRIPTION DÉTAILLÉE:}

Contient toute l'information nécessaires pour facturer un déboursé au client pour lequel le déboursé à été effectué. L'écriture est imputée à l'auxiliaire des travaux en cours et, afin de reconnaître les déboursés, le chiffre 999 est utilisé comme numéro d'employé et le code d'activité employé est le "980".

STRUCTURE DE DONNÉES INCLUSES:

Nom du client

Description de la facture

Numéro du client

Code d'activité

Montant.

VOLUME:

Environ 200 par mois. 
Flux de données

\section{Feuilles de déboursés, D5.5/6-e}

REF. SOURCE: D5.5/6

REF. DESTIN.: e
DESCRIPTION: Feuilles de déboursés

DESCRIPTION: Société d'informatique de gestion

\section{DESCRIPTION DÉTAILLÉE:}

Contient le nom du client, la description du déboursé, le numéro du client, le numéro du code d'activité et le montant. Cette feuille est envoyée à la Société d'informatique de gestion pour mettre à jour l'auxiliaire des travaux en cours. Sur le rapport de l'auxiliaire des travaux en cours, le code 980 représente un déboursé facturé au client. On utilise le numéro d'employé 999 pour enregistrer les déboursés facturables.

\section{STRUCTURE DE DONNÉES INCLUSES:}

Numéro d'employé: toujours 999

Nom de l'entreprise: Harel Drouin Ass. ou Harel Drouin Gestion Conseil

Date: date à laquelle sont inscrites les données

Nom du client

Description

Numéro du client

Code d'activité: toujours 980

Le montant.

VOLUME:

6 feuilles par mois. 
Flux de données

\section{Factures imputées, 5.5.4-5.5.5}

REF. SOURCE: $\mathbf{5 . 5 . 4}$

DESCRIPTION: Préparer chèques et enveloppes

REF. DESTIN.: $\mathbf{5 . 5 . 5}$

DESCRIPTION: Signer les chèques

DESCRIPTION DÉTAILLÉE:

Factures contenant un ou plusieurs codes d'imputation.

STRUCTURE DE DONNÉES INCLUSES:

Nom du fournisseurs

Description des items achetés

Quantité des items achetés

Total de la facture

Autorisation

Code(s) d'imputation

Montant(s) à imputer.

VOLUME:

Environ 400 par mois. 
Flux de données

Copies blanches des chèques et talon, 5.5.4-5.5.5

REF. SOURCE: $\mathbf{5 . 5 . 4}$

DESCRIPTION: Préparer chèques et enveloppes

REF. DESTIN.: $\mathbf{5 . 5 . 5}$

DESCRIPTION: Signer les chèques

\section{DESCRIPTION DÉTAILLÉE:}

Copie blanche du chèque et du talon.

STRUCTURE DE DONNÉES INCLUSES:

Partie chèque:

- Numéro du chèque

- Nom de l'émetteur

- Adresse de l'émetteur

- Montant du chèque inscrit à l'aide du protectographe

- Montant du chèque dactylographié

- Date du chèque

- Nom du destinataire

- Mention "non négociable"

- Nom de la banque

- Adresse de la banque

Partie talon:

- Date du chèque

- Code(s) d'imputation

- Montant(s) imputé(s)

- Nom du fournisseurs

- Information si à chargé ou déjà charger.

VOLUME:

400 par mois. 
Flux de données

Copies blanches des chèques et talons, 5.5.4-5.5.5

REF. SOURCE: $\mathbf{5 . 5 . 4}$

DESCRIPTION: Préparer chèques et enveloppes

REF. DESTIN.: $\mathbf{5 . 5 . 5}$

DESCRIPTION: Signer les chèques

DESCRIPTION DÉTAILLÉE:

Copie du chèque et du talon.

STRUCTURE DE DONNÉES INCLUSES:

Partie chèque:

- Numéro du chèque

- Nom de l'émetteur

- Adresse de l'émetteur

- Montant du chèque inscrit à l'aide du protectographe

- Montant du chèque dactylographié

- Date du chèque

- Nom du destinataire

- Mention "non négociable"

- Nom de la banque

- Adresse de la banque

Partie talon:

- Date du chèque

- Code(s) d'imputation

- Montant(s) imputé(s)

- Nom du fournisseurs

- Information si à chargé ou déjà charger.

VOLUME:

400 par mois. 
Flux de données

\section{Copies jaunes des chèques et talons, 5.5.4-5.5.5}

REF. SOURCE: 5.5 .4

DESCRIPTION: Préparer chèques et enveloppes

REF. DESTIN.: 5.5 .5

DESCRIPTION: Enregistrer les chèques

\section{DESCRIPTION DÉTAILLÉE:}

Copie blanche du chèque et du talon.

STRUCTURE DE DONNÉES INCLUSES:

Partie chèque:

- Numéros du chèque

- Nom de l'émetteur

- Adresse de l'émetteur

- Montant du chèque inscrit à l'aide du protectographe

- Montant du chèque dactylographié

- Date du chèque

- Nom du destinataire

- Mention "non négociable"

- Nom de la banque

- Adresse de la banque

Partie talon:

- Date du chèque

- Code(s) d'imputation

- Montant(s) imputé(s)

- Nom du fournisseurs

- Information si à chargé ou déjà charger.

VOLUME:

Environ 400 par mois. 
Flux de données

\section{Copies jaunes des chèques et talons, 5.5.6-5.5.7}

REF. SOURCE: $\mathbf{5 . 5 . 6}$

DESCRIPTION: Enregistrer les chèques

REF. DESTIN.: 5.5 .7

DESCRIPTION: Faire un total de contrôle

DESCRIPTION DÉTAILLÉE:

Copie blanche du chèque et du talon.

STRUCTURE DE DONNÉES INCLUSES:

Partie chèque:

- Numéros du chèque

- Nom de l'émetteur

- Adresse de l'émetteur

- Montant du chèque inscrit à l'aide du protectographe

- Montant du chèque dactylographié

- Date du chèque

- Nom du destinataire

- Mention "non négociable"

- Nom de la banque

Partie talon:

- Adresse de la banque

- Date du chèque

- Code(s) d'imputation

- Montant(s) imputé(s)

- Nom du fournisseurs

- Information si à chargé ou déjà charger.

VOLUME:

400 par mois. 
Flux de données

\section{Copies jaunes, 5.5.7-e}

REF. SOURCE: $\mathbf{5 . 5 . 7}$

REF. DESTIN.: e
DESCRIPTION: Faire un total de contrôle

DESCRIPTION: Société d'informatique de gestion

\section{DESCRIPTION DÉTAILLÉE:}

Copie du chèque et du talon. Le papier sur lequel est effectué cette copie est blanc.

STRUCTURE DE DONNÉES INCLUSES:

Partie chèque:

- Numéros du chèque

- Nom de l'émetteur

- Adresse de l'émetteur

- Montant du chèque inscrit à l'aide du protectographe

- Montant du chèque dactylographié

- Date du chèque

- Nom du destinataire

- Mention "non négociable"

- Nom de la banque

Partie talon:

- Adresse de la banque

- Date du chèque

- Code(s) d'imputation

- Montant(s) imputé(s)

- Nom du fournisseurs

- Information si à chargé ou déjà charger.

VOLUME:

400 par mois. 
Flux de données

\section{Total de contrôle, 5.5.7-e}

REF. SOURCE: $\mathbf{5 . 5 . 7}$

REF. DESTIN.: $e$
DESCRIPTION: Faire un total de contrôle DESCRIPTION: Société d'informatique de gestion

\section{DESCRIPTION DÉTAILLÉE:}

Total de toutes les copies jaunes qui sont envoyées à la Société d'informatique de gestion.

STRUCTURE DE DONNÉES INCLUSES:

Total.

VOLUME:

1 par lot de copies jaunes. 
Flux de données

\section{Chèques et pièces, 5.5.5-d}

REF. SOURCE: $\mathbf{5 . 5 . 5}$

DESCRIPTION: Signer les chèques

REF. DESTIN.: d

DESCRIPTION: Fournisseurs

\section{DESCRIPTION DÉTAILLÉE:}

Chèques pour payer les factures.

STRUCTURE DE DONNÉES INCLUSES:

- Nom de l'émetteur, son adresse, numéro du chèque, date (jour, mois, année)

- Son adresse

- Numéro du chèque

- Date (jour, mois, année)

- Nom du destinataire

- Montant (en chiffres)

- Montant inscrit avec le protectographe

- Signatures (2)

- Nom de la banque

- Adresse de la banque.

\section{VOLUME:}

Environ $\mathbf{4 0 0}$ par mois. 
Flux de données

\section{Factures, 5.5.5-D5.5/5}

REF. SOURCE: $\mathbf{5 . 5 . 5}$

REF. DESTIN.: D5.5/5
DESCRIPTION: Signer les chèques

DESCRIPTION: Factures payées

\section{DESCRIPTION DÉTAILLÉE:}

Contient l'ensemble des factures qui seront payées. Toutes ces factures ont été autorisées sauf celles concernant la location de certaines équipements ou représentant des mensualités. Il est impossible de connaître le nombre de factures payées dans un mois. Cependant, nous pouvons affirmer qu'il y a plus de 400 factures qui sont payées car nous produisons environ 400 chèques par mois.

\section{STRUCTURE DE DONNÉES INCLUSES:}

Nom du fournisseurs

Description des items achetés

Quantité

Total de la facture

Autorisation (selon le type de facture)

Code(s) d'imputation

Montant(s) à imputer.

\section{VOLUME:}

Plus de $\mathbf{4 0 0}$ par mois. 
Flux de données

\section{Copies blanches des chèques et talons, 5.5.5-D5.5/5}

REF. SOURCE: $\mathbf{5 . 5 . 5}$

DESCRIPTION: Signer les chèques

REF. DESTIN.: D5.5/5

DESCRIPTION: Factures payées

DESCRIPTION DÉTAILLÉE:

Copie blanche du chèque et du talon.

STRUCTURE DE DONNÉES INCLUSES:

Partie chèque:

- Numéro du chèque

- Nom de l'émetteur

- Adresse de l'émetteur

- Montant du chèque inscrit à l'aide du protectographe

- Montant du chèque dactylographié

Partie talon:

- Date du chèque

- Nom du destinataire

- Mention "non négociable"

- Nom de la banque

- Adresse de la banque

- Date du chèque

- Code(s) d'imputation

- Montant(s) imputé(s)

- Nom du fournisseur

- Information si déjà facturé ou à facturé. 


\section{INDEX}

\section{Processus 2.0, Compiler le temps}

Diagrammes de flux de données $\ldots \ldots \ldots \ldots \ldots \ldots \ldots, 2$

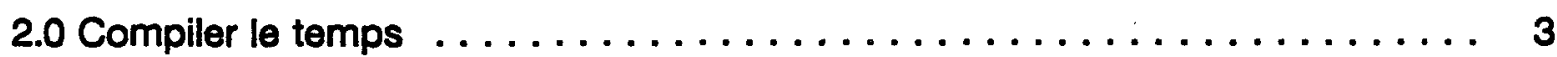

2.7 Vérifier le sommaire $\ldots \ldots \ldots \ldots \ldots \ldots \ldots \ldots \ldots \ldots \ldots \ldots \ldots$

Processus de traitement $\ldots \ldots \ldots \ldots \ldots \ldots \ldots \ldots \ldots \ldots \ldots \ldots$

Approuver la feuille de temps, $2.10 \ldots \ldots \ldots \ldots \ldots \ldots \ldots \ldots \ldots \ldots \ldots$

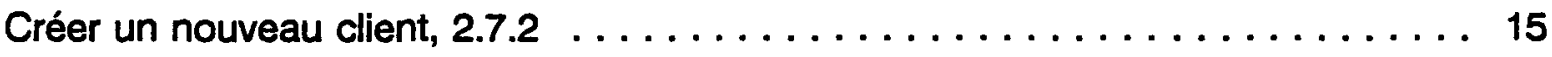

Enregistrer les données pour la feuille de temps, $2.2 \ldots \ldots \ldots \ldots \ldots \ldots \ldots 7$

Enregistrer les données de base, $2.1 \ldots \ldots \ldots \ldots \ldots \ldots \ldots \ldots \ldots \ldots$

Enregistres les numéros manquants $2.7 .1 . \ldots \ldots \ldots \ldots \ldots \ldots \ldots \ldots \ldots \ldots 14$

Imprimer la feuille de temps, $2.3 \ldots \ldots \ldots \ldots \ldots \ldots \ldots \ldots \ldots \ldots 8$

Procéder à la fermeture du mois, $2.9 \ldots \ldots \ldots \ldots \ldots \ldots \ldots \ldots \ldots \ldots$

Procéder à la fermeture de la semaine, $2.5 \ldots \ldots \ldots \ldots \ldots \ldots \ldots \ldots \ldots 11$

Produire le sommaire mensuel, $2.6 \ldots \ldots \ldots \ldots \ldots \ldots \ldots \ldots \ldots \ldots \ldots \ldots \ldots$

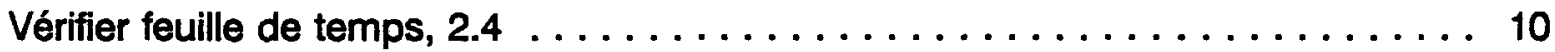

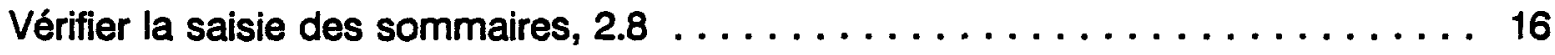

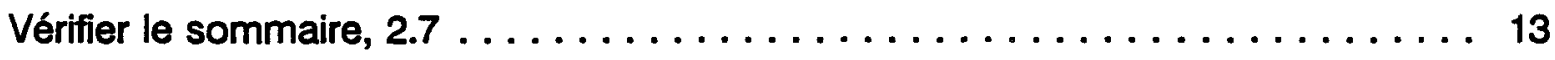

Flux de données $\ldots \ldots \ldots \ldots \ldots \ldots \ldots \ldots \ldots \ldots \ldots \ldots$

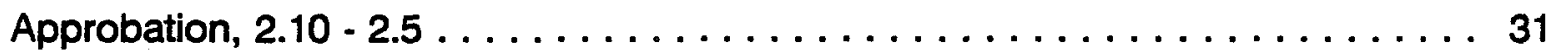

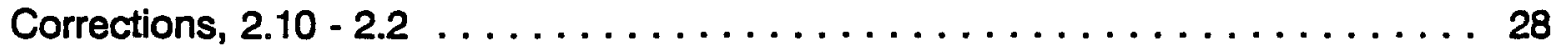

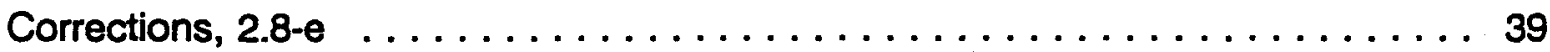

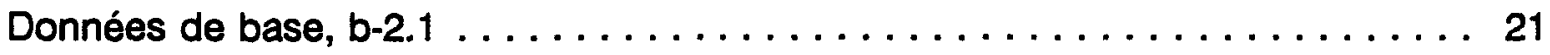

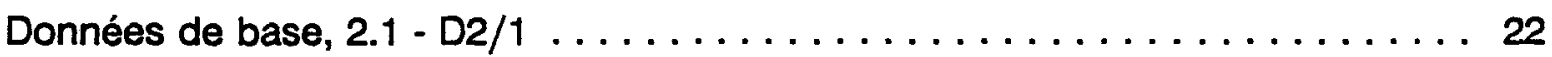

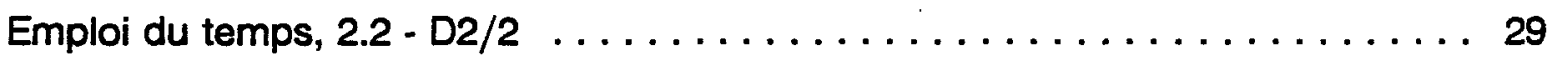

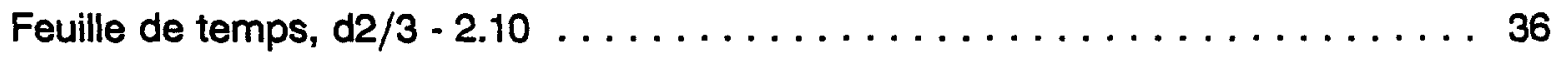




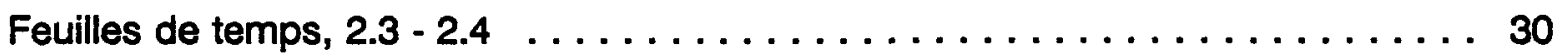

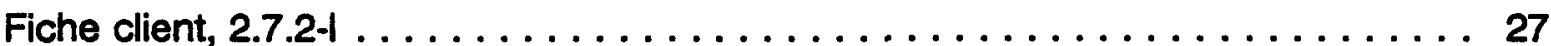

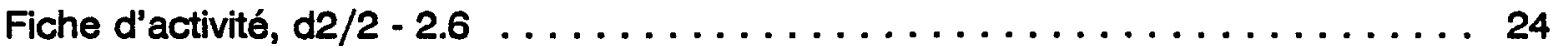

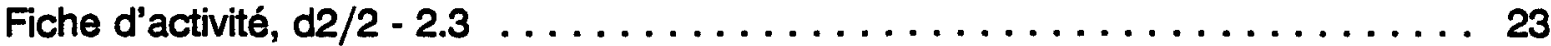

Heures facturables et non facturables $2.9-02 / 5 \ldots \ldots \ldots \ldots \ldots$

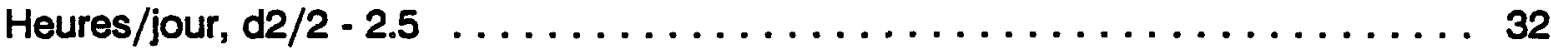

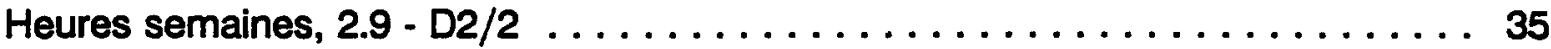

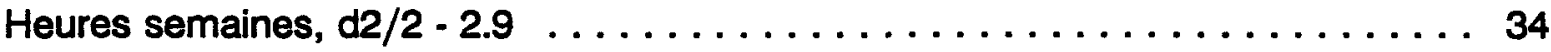

Nom et temps du dossier, b-2.2 . . . . . . . . . . . . . . . . . . . 20

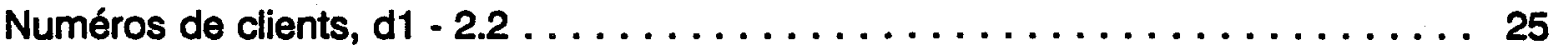

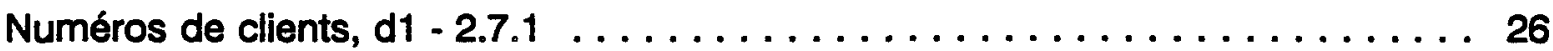

Sommaire mensuel, $2.6-2.7 \ldots \ldots \ldots \ldots \ldots \ldots \ldots \ldots \ldots \ldots \ldots$

Validation des feuilles de temps, $e-2.8 \ldots \ldots \ldots \ldots \ldots \ldots \ldots$

\section{Processus 5.1, Enregistrer les ventes ...... 40}

Diagrammes de flux de données .................41

5.1 Enregistrer les ventes $\ldots \ldots \ldots \ldots \ldots \ldots \ldots \ldots \ldots \ldots \ldots \ldots \ldots \ldots \ldots$

Processus de traitement $\ldots \ldots \ldots \ldots \ldots \ldots \ldots \ldots \ldots \ldots \ldots 43$

Additionner le copies de factures, $5.1 .6 \ldots \ldots \ldots \ldots \ldots \ldots$

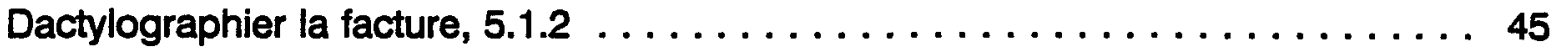

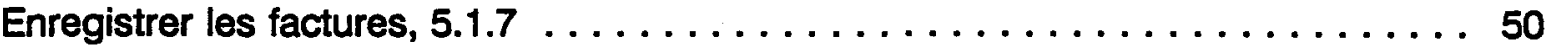

Numéroter les copies de factures, $5.1 .5 \ldots \ldots \ldots \ldots \ldots \ldots \ldots \ldots$

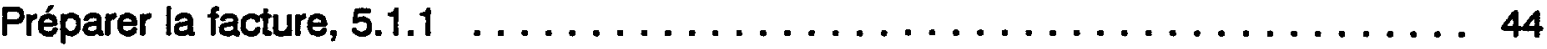

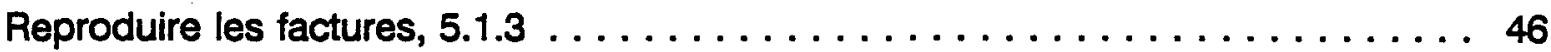

Vérifier l'enregistrement des factures, $5.1 .8 \ldots \ldots \ldots \ldots \ldots \ldots \ldots \ldots \ldots \ldots \ldots \ldots$

Vérifier les copies de factures, $5.1 .4 \ldots \ldots \ldots \ldots \ldots \ldots \ldots \ldots \ldots$

Flux de données ............................. 52

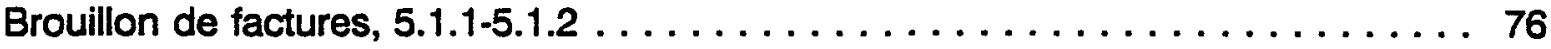

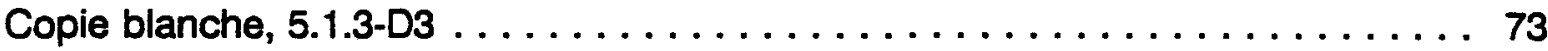

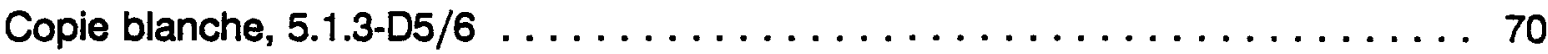


Copie blanche, $5.1 .3-05 / 7 \ldots \ldots \ldots \ldots \ldots$

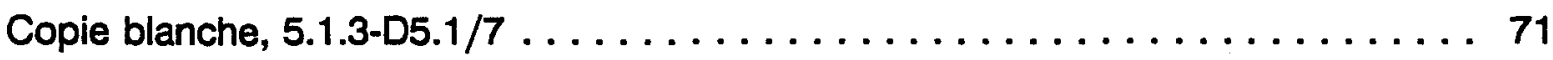

Copie facture antérieure, d3-5.1.1 . . . . . . . . . . . . . . 81

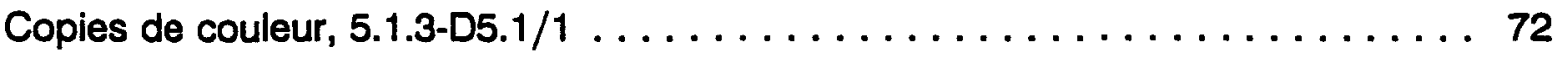

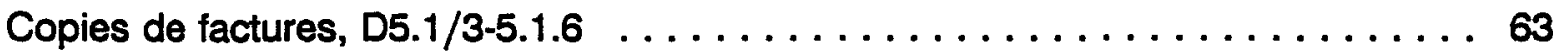

Copies des factures, $5.1 .5-D 5.1 / 3 \ldots \ldots \ldots \ldots \ldots$

Copies des factures, $05.1 / 5-5.1 .5 \ldots \ldots \ldots \ldots \ldots 6$

Copies des factures, $05.1 / 1-5.1 .4 \ldots \ldots \ldots \ldots \ldots$. . . . . . . . . . 68

Copies des factures, $5.1 .4-D 5.1 / 5 \ldots \ldots \ldots \ldots$. . . . . . . . . . . . . . . 67

Copies des factures, $5.1 .6-D 5.1 / 8 \ldots \ldots \ldots \ldots$. . . . . . . . . . . . . . 60

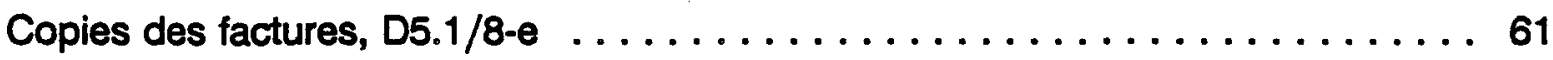

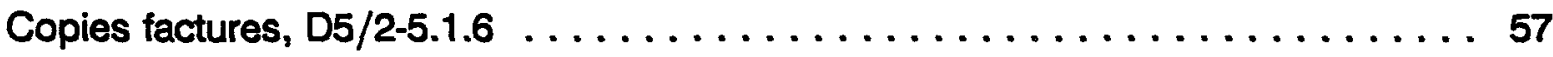

Copies vertes des factures $05.1 / 3-5.1 .7 \ldots \ldots \ldots \ldots \ldots \ldots \ldots$

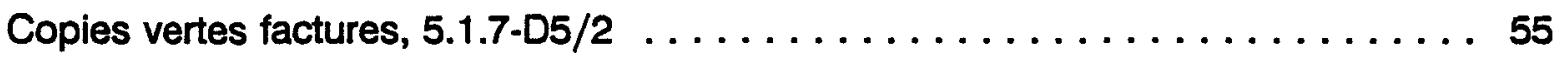

Copies vertes factures, $5.1 .7-D 5.1 / 4 \ldots \ldots \ldots \ldots \ldots \ldots$

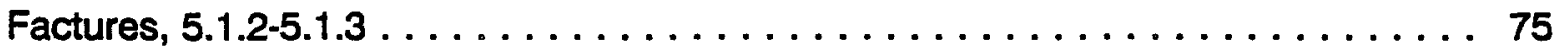

Factures, $5.1 .3-a \ldots \ldots \ldots \ldots \ldots \ldots \ldots \ldots \ldots \ldots \ldots \ldots \ldots \ldots$

Formulaires de transaction, $5.1 .6-\mathrm{D} 5 / 8 \ldots \ldots \ldots \ldots \ldots \ldots$

Formulaires de transactions, $D 5 / 8-e \ldots \ldots \ldots \ldots \ldots \ldots \ldots$

Montant imputé, D5/6-5.1.1 . . . . . . . . . . . . . . . . 80

Montants, $05.1 / 4-5.1 .6 \ldots \ldots \ldots \ldots \ldots 2$

Nombre d'heures, b-5.1.1 . . . . . . . . . . . . . . . . . . . 78

Taux horaire, $\mathrm{D} 5 / 6-5.1 .1 \ldots \ldots \ldots \ldots \ldots$. . . . . . . . . . . . . . . 79

Total copies vertes acceptées . . . . . . . . . . . . . . . . . . . . . . . 54

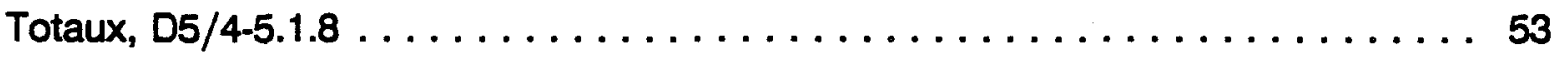

Travail effectué, b-5.1.1 $\ldots \ldots \ldots \ldots \ldots \ldots \ldots \ldots$ 


\section{Processus 5.2, Enregistrer les recettes .... 82} Diagrammes de flux de données ................... 83

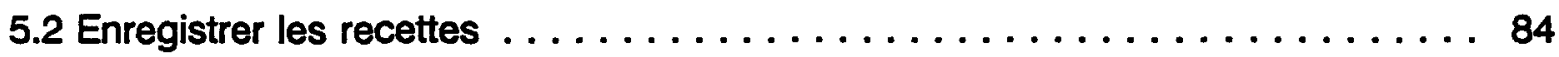

Processus de traitement $\ldots \ldots \ldots \ldots \ldots \ldots \ldots \ldots \ldots \ldots, 85$

Additionner les montants, $5.2 .3 \ldots \ldots \ldots \ldots \ldots \ldots$. . . . . . . . . 88

Comparer les soldes, $5.2 .3 \ldots \ldots \ldots \ldots \ldots \ldots \ldots \ldots \ldots$

Enregistrer le numéro de la facture, $5.2 .5 \ldots \ldots \ldots \ldots \ldots \ldots$

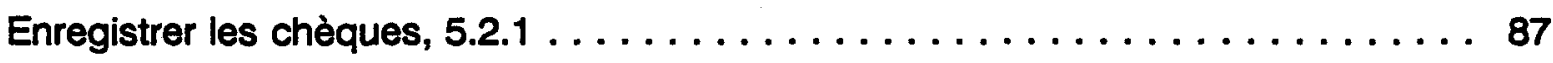

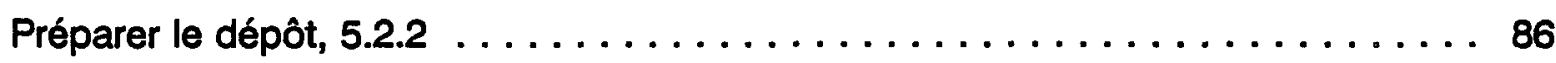

Flux de données .............................. 91

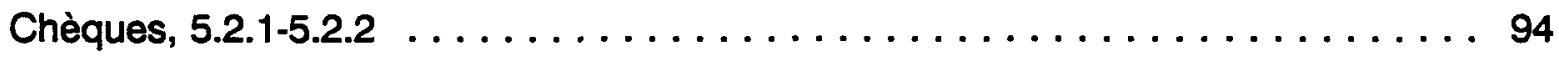

Chèques, $5.2 .1-D 5 / 7 \ldots \ldots \ldots \ldots \ldots \ldots \ldots \ldots \ldots \ldots \ldots \ldots$

Chèques, $5.2 .1-D 5 / 7 \ldots \ldots \ldots \ldots \ldots \ldots \ldots \ldots \ldots \ldots \ldots$

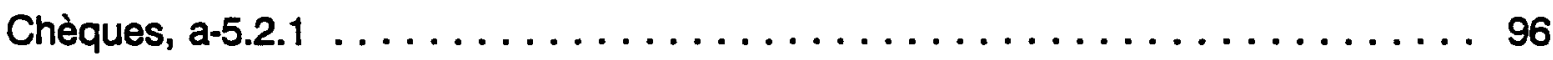

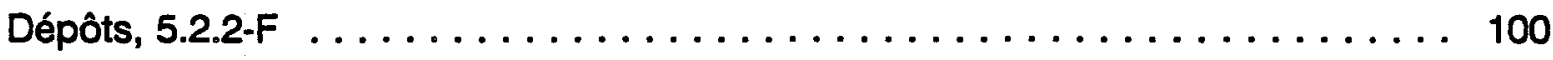

Formulaire de transactions, $D 5 / 8-e \ldots \ldots \ldots \ldots \ldots \ldots \ldots$

Information relative au chèque, $5.2 .1-D 5 / 8 \ldots \ldots \ldots \ldots \ldots$

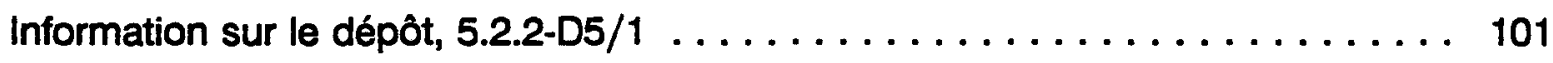

Montant, D5/8-5.2.3 . . . . . . . . . . . . . . . . . . . . . . 108

Montant du dépôt, $5.2 .2-5.2 .4 \ldots \ldots \ldots \ldots \ldots \ldots$. . . . . . . . . . . . . . 99

Nom du client, $\mathrm{f}-5.2 .1 \ldots \ldots \ldots \ldots \ldots \ldots \ldots$

Numéro de facture, $D 5 / 5-5.2 .5 \ldots \ldots \ldots \ldots \ldots \ldots \ldots$. . . . . . . . . . . 104

Numéro de facture, $\mathrm{D} 5 / 2-5.2 .5 \ldots \ldots \ldots \ldots \ldots \ldots \ldots \ldots \ldots$

Numéro de facture, $5.2 .5-05 / 8 \ldots \ldots \ldots \ldots \ldots \ldots$. . . . . . . . . . . 102

Numéro du client, dt-5.2.1 . . . . . . . . . . . . . . . . . . 92

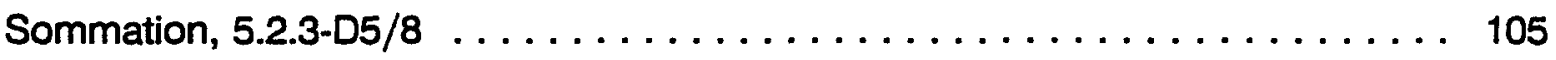

Sommation des transactions, $5.2 .3 \ldots \ldots \ldots \ldots \ldots$. . . . . . . . . . 107 


\section{Processus 5.4, Concilier les comptes de} banque

Diagrammes de flux de données 109

5.4 Concilier les comptes de banque 110 111

Processus de traitement $\ldots \ldots \ldots \ldots \ldots \ldots \ldots \ldots \ldots \ldots \ldots, 112$

Calculer le solde au livre, $5.4 .4 \ldots \ldots \ldots \ldots \ldots \ldots \ldots \ldots \ldots \ldots \ldots$

Comparer les soldes aux livres, $5.4 .5 \ldots \ldots \ldots \ldots \ldots \ldots \ldots \ldots \ldots \ldots \ldots 117$

Compiler les transactions enregistrées, $5.4 .6 \ldots \ldots \ldots \ldots \ldots \ldots \ldots \ldots \ldots 118$

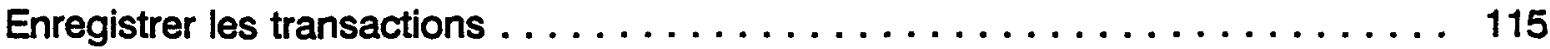

Identifier les chèques et dépôts $\ldots \ldots \ldots \ldots \ldots \ldots \ldots \ldots \ldots \ldots \ldots \ldots 114$

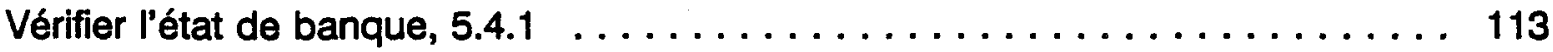

Flux de données ............................. 119

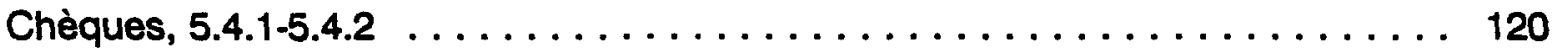

Chèques et dépôts en circulation, 5.4.2-D5.4/3 $\ldots \ldots \ldots \ldots \ldots \ldots \ldots \ldots 132$

Chèques et dépôts en circulation, $05.4 / 2-5.4 .2 \ldots \ldots \ldots \ldots \ldots \ldots \ldots \ldots 131$

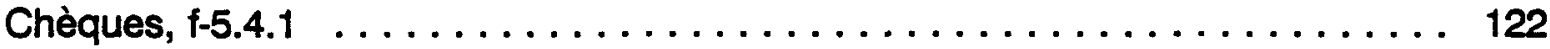

Copie jaune chèque et talon, $05.4 / 1-5.4 .2 \ldots \ldots \ldots \ldots \ldots \ldots \ldots \ldots \ldots 130$

Copies jaunes des chèques et talon, e-D5.4/1 $\ldots \ldots \ldots \ldots \ldots \ldots \ldots \ldots 143$

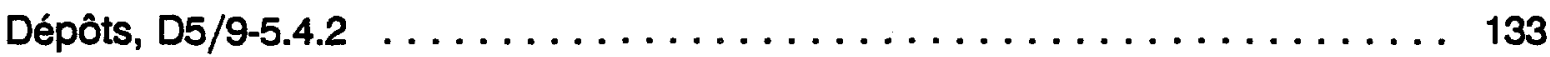

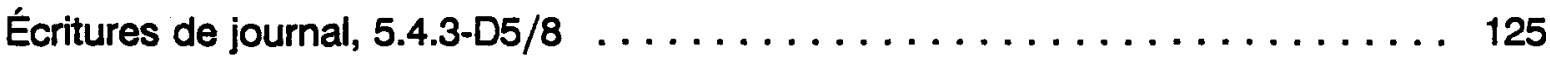

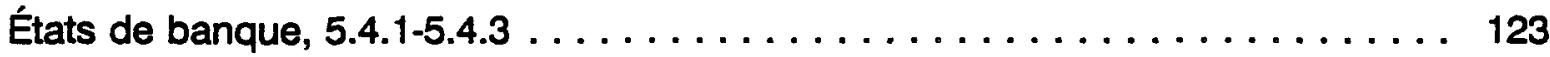

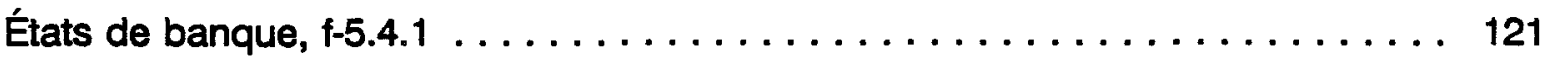

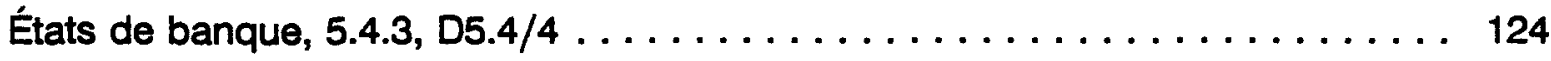

Formulaires de transactions, $D 5 / 8-e \ldots \ldots \ldots \ldots \ldots \ldots \ldots \ldots \ldots \ldots . \ldots \ldots$

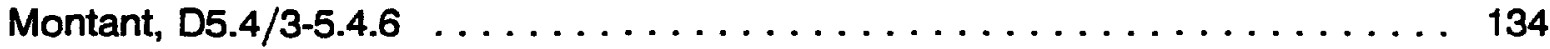

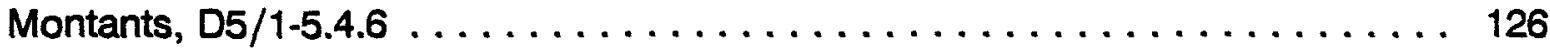

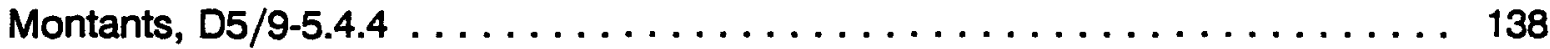

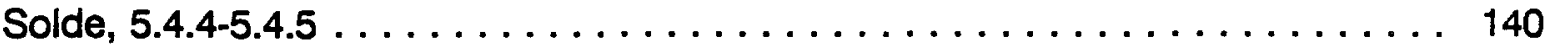


Solde au livre, $5.4 .4-D 5.4 / 3 \ldots \ldots \ldots \ldots \ldots \ldots \ldots \ldots \ldots$

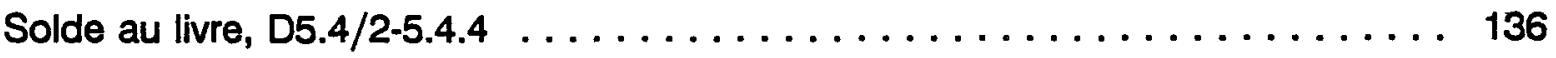

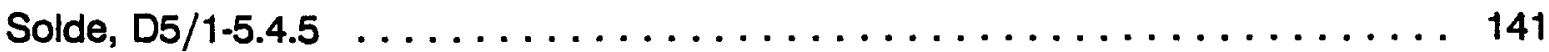

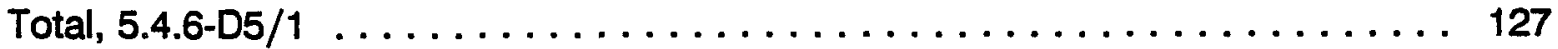

Total des chèques, e-5.4.4 . . . . . . . . . . . . . . . . . . . . . 137

Totaux, 5.4.6-D5.4/3 . . . . . . . . . . . . . . . . . . . 135

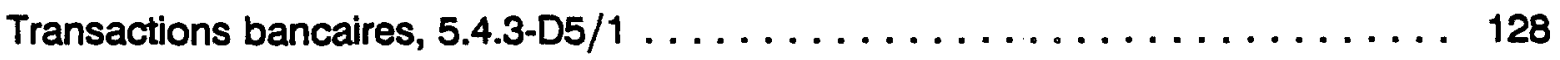

Transactions bancaires, $5.4 .3-D 5.4 / 3 \ldots \ldots \ldots \ldots \ldots \ldots \ldots$

\section{Processus 5.5, Payer les factures des fournisseurs ..................................... 144}

Diagrammes de flux de données ................. 145

5.4 Payer les fournisseurs $\ldots \ldots \ldots \ldots \ldots \ldots \ldots \ldots \ldots \ldots$

Processus de traitement $\ldots \ldots \ldots \ldots \ldots \ldots \ldots \ldots \ldots \ldots \ldots \ldots \ldots$

Autoriser les factures, $5.5 .1 \ldots \ldots \ldots \ldots \ldots \ldots$

Enregistrer les chèques, $5.5 .6 \ldots \ldots \ldots \ldots \ldots \ldots \ldots \ldots \ldots \ldots$

Faire un total de contrôle, $5.5 .7 \ldots \ldots \ldots \ldots \ldots \ldots \ldots \ldots \ldots \ldots$

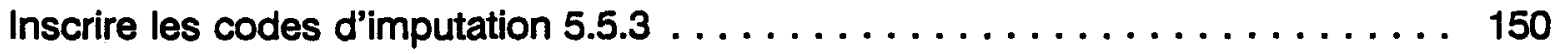

Préparer les chèques et enveloppes, $5.5 .4 \ldots \ldots \ldots \ldots \ldots \ldots 15 \ldots \ldots \ldots$

Sélectionner les factures qui seront payées, $5.5 .2 \ldots \ldots \ldots$. . . . . . . . . . 149

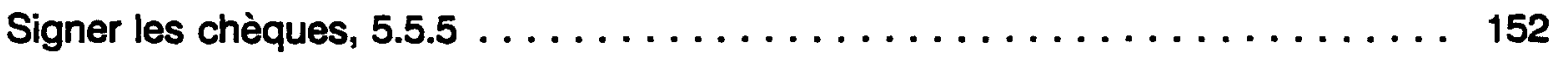

Flux de données .......................... 155

Autorisation, $b-5.5 .1 \ldots \ldots \ldots \ldots \ldots \ldots \ldots \ldots \ldots \ldots$

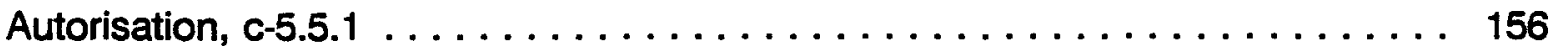

Chèques et pièces, $5.5 .5-d \ldots \ldots \ldots \ldots \ldots \ldots \ldots \ldots \ldots \ldots$

Copies blanches des chèques et talons, 5.5.4-5.5.5 . . . . . . . . . . 168

Copies blanches des chèques et talon, $5.5 .4-5.5 .5 \ldots \ldots \ldots \ldots 7$

Copies blanches des chèques et talons, 5.5.5-D5.5/5 . . . . . . . . . 175

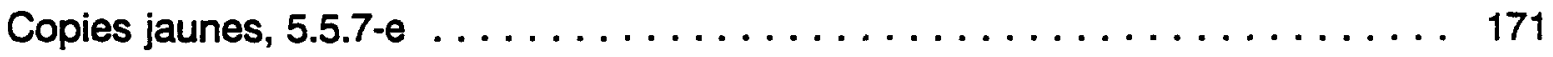


Copies jaunes des chèques et talons, $5.5 .4-5.5 .5 \ldots \ldots \ldots \ldots \ldots \ldots \ldots 169$

Copies jaunes des chèques et talons, $5.5 .6-5.5 .7 \ldots \ldots \ldots \ldots \ldots \ldots \ldots \ldots$

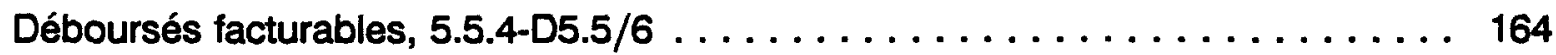

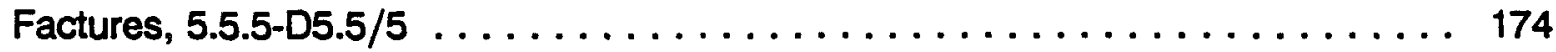

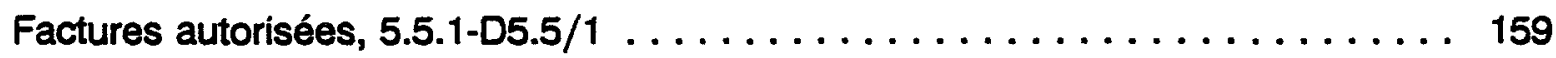

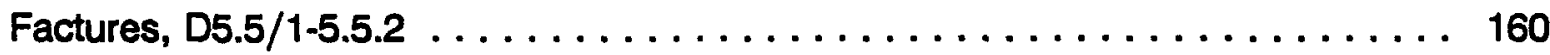

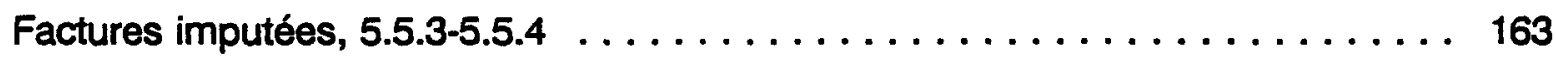

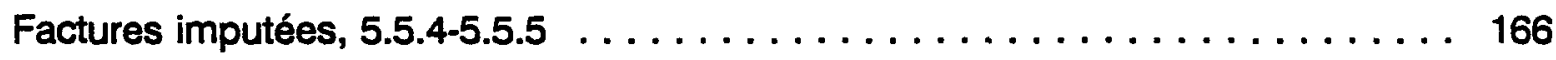

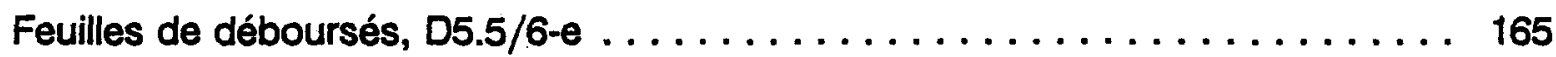

Numéro de compte, D5.5/4-5.5.3 ..................... 162

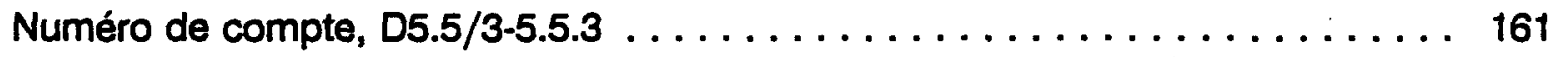

Numéro du client, c-5.5.1 ........................ 157

Total de contrôle, $5.5 .7-e \ldots \ldots \ldots \ldots \ldots \ldots \ldots \ldots \ldots \ldots \ldots \ldots . \ldots \ldots \ldots \ldots$ 
Annexe II

\section{Dictionnaire}

Nouveau système 


\section{TABLE DES MATIÈRES}

\section{Processus 2.0, Compiler feuille de temps 1}

Diagramme de flux d'information ................... 2

2.0 Compiler feuille de temps $\ldots \ldots \ldots \ldots \ldots \ldots \ldots \ldots \ldots \ldots \ldots \ldots$

Processus de traitement $\ldots \ldots \ldots \ldots \ldots \ldots \ldots \ldots \ldots \ldots, 4$

Produire les feuilles de temps, $2.1 \ldots \ldots \ldots \ldots \ldots \ldots \ldots \ldots \ldots$

Saisir données feuilles de temps, $2.8 \ldots \ldots \ldots \ldots \ldots \ldots \ldots \ldots \ldots \ldots \ldots \ldots \ldots$

Valider feuilles de temps, $2.2 \ldots \ldots \ldots \ldots \ldots \ldots \ldots \ldots \ldots \ldots \ldots$

Corriger les transactions, $2.3 \ldots \ldots \ldots \ldots \ldots \ldots \ldots \ldots \ldots \ldots \ldots \ldots \ldots \ldots$

Créer un nouveau client, $2.4 \ldots \ldots \ldots \ldots \ldots \ldots \ldots \ldots \ldots \ldots \ldots$

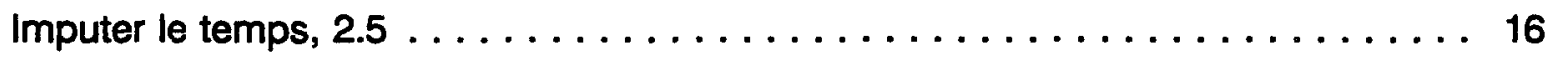

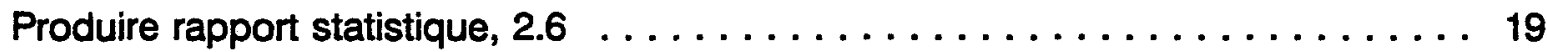

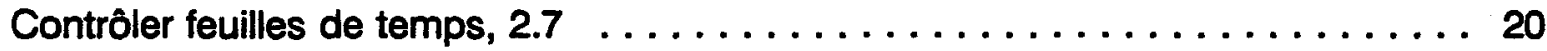

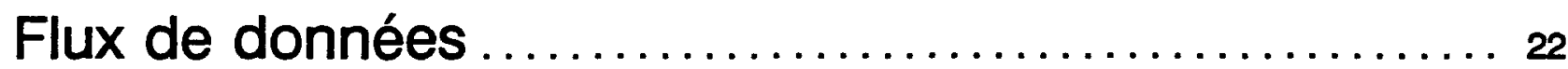

Données de feuilles de temps, b-2.1 ................... 23

Transactions, b-2.8 . . . . . . . . . . . . . . . . . . . . . . . 24

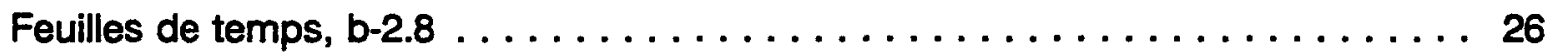

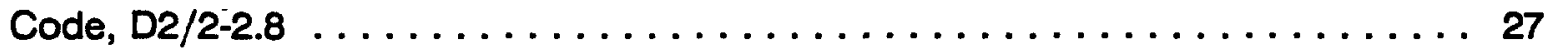

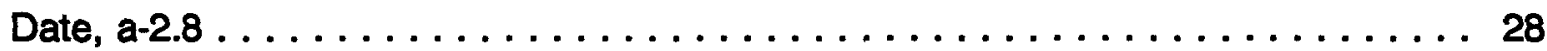

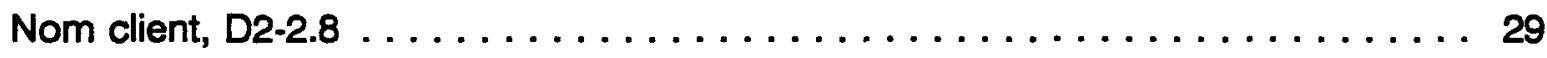

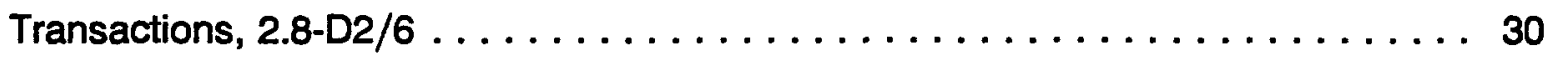

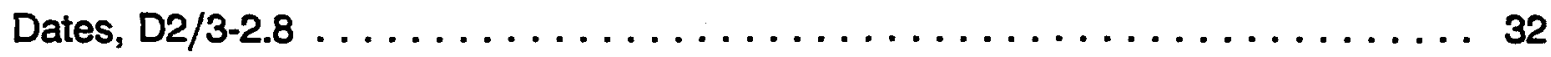

Numéro, $2.2-\mathrm{D} 2 \ldots \ldots \ldots \ldots \ldots \ldots \ldots \ldots \ldots \ldots \ldots \ldots \ldots \ldots \ldots$

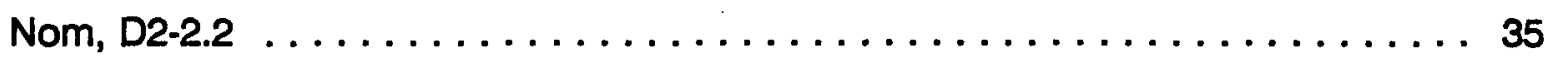


Transactions, $2.2-\mathrm{D} 2 / 6 \ldots \ldots \ldots \ldots \ldots \ldots \ldots \ldots \ldots \ldots \ldots \ldots \ldots \ldots \ldots \ldots \ldots$

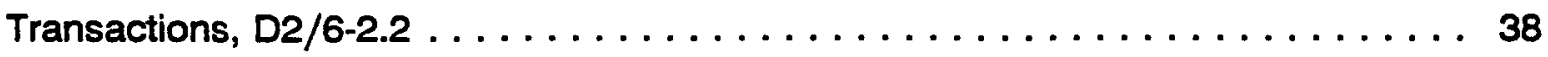

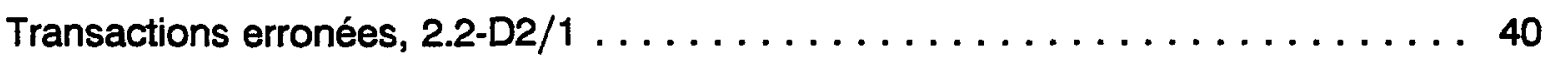

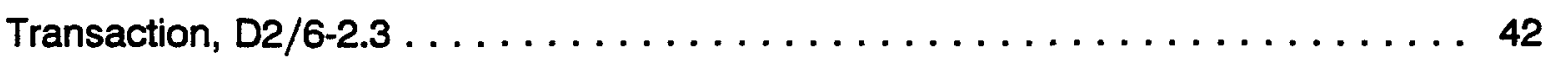

Code, $2.3-D 2 / 2 \ldots \ldots \ldots \ldots \ldots \ldots \ldots \ldots \ldots \ldots \ldots \ldots \ldots \ldots \ldots 44$

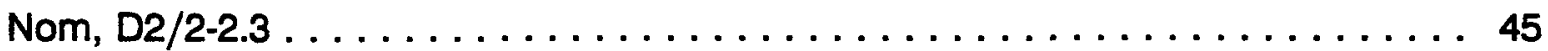

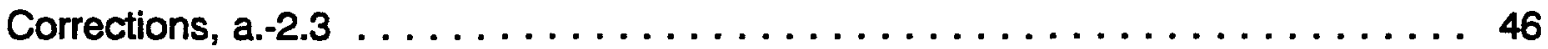

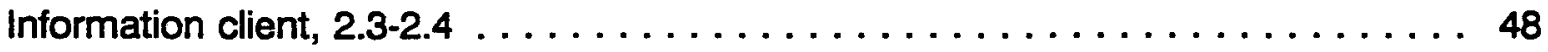

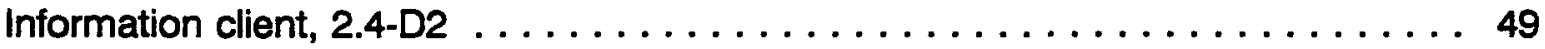

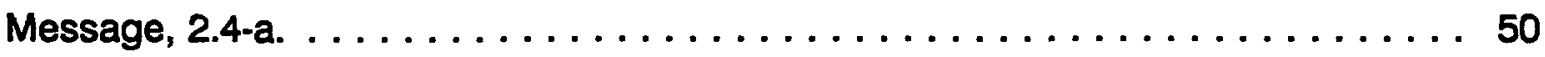

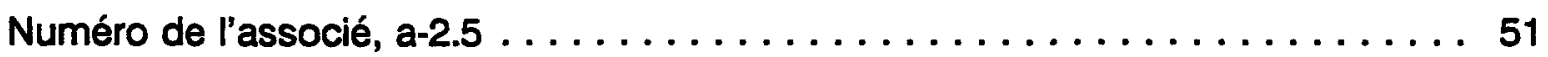

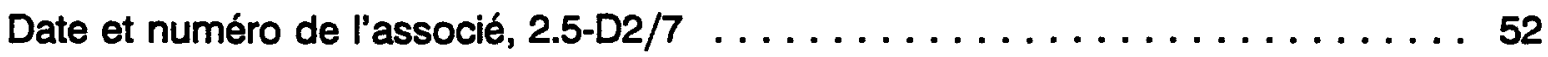

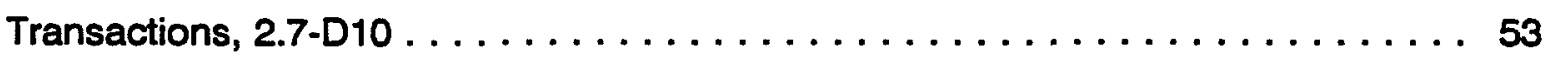

Transactions facturables, $2.5-\mathrm{D} 7 \ldots \ldots \ldots \ldots \ldots \ldots \ldots \ldots \ldots \ldots \ldots \ldots$

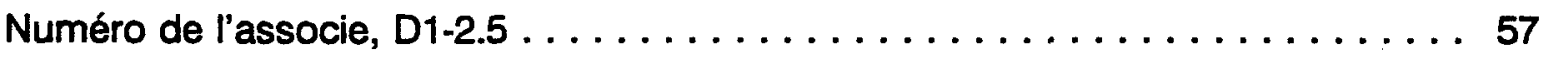

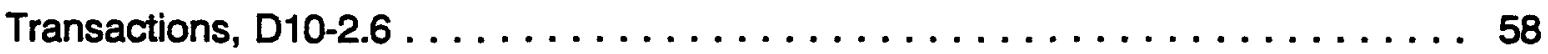

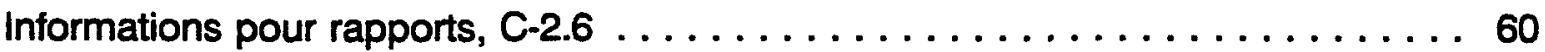

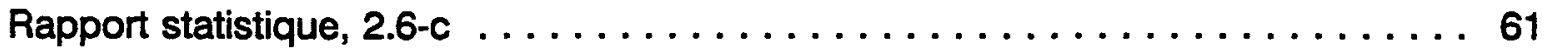

Coordonnées, D2/3-2.7 .........................62

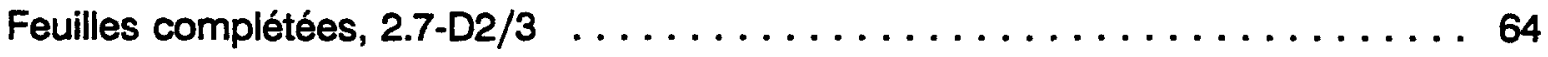

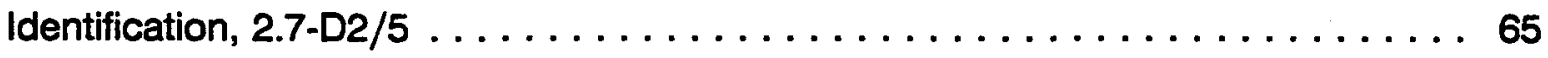

Feuilles de temps manquantes, $2.7-A \ldots \ldots \ldots \ldots \ldots \ldots \ldots \ldots \ldots 67$

Date de la feuille de temps, A-2.7 ..................... 69

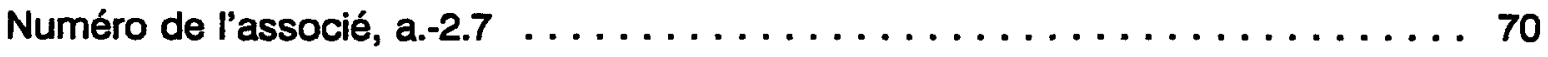

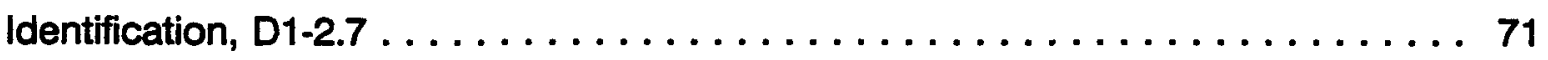

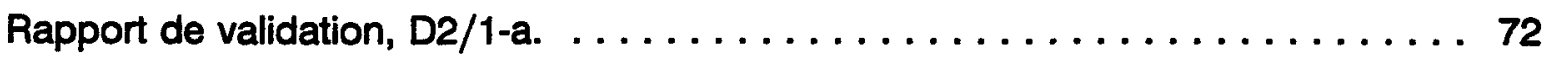

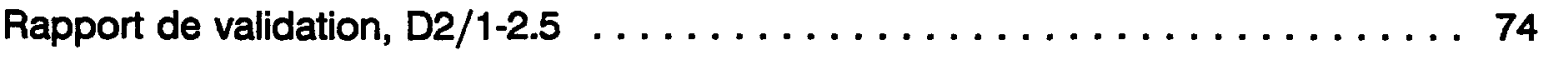

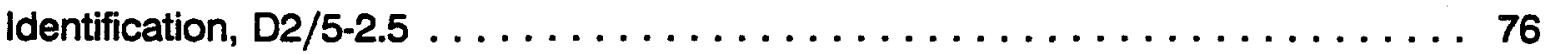

Temps supplémentaire, $2.7-\mathrm{D} 2 / 4 \ldots \ldots \ldots \ldots \ldots \ldots \ldots \ldots \ldots \ldots \ldots$

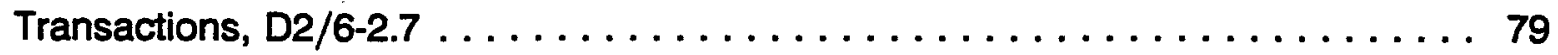

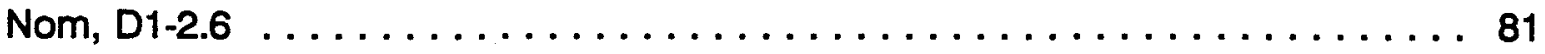

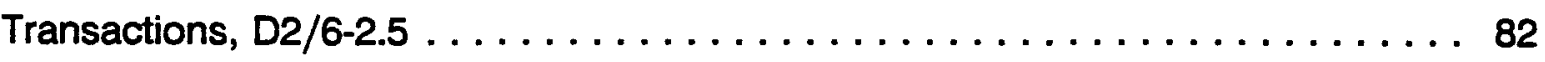

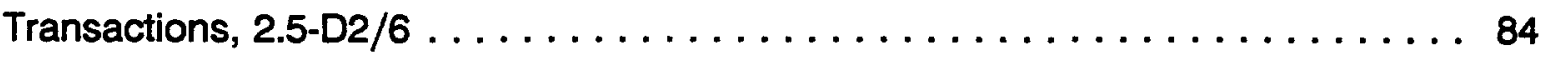

Information client, a.-2.4 $\ldots \ldots \ldots \ldots \ldots \ldots \ldots \ldots \ldots \ldots \ldots \ldots \ldots \ldots$ 
Transaction, $2.3-\mathrm{D} 2 / 6 \ldots \ldots \ldots \ldots \ldots \ldots \ldots \ldots \ldots \ldots \ldots \ldots \ldots$

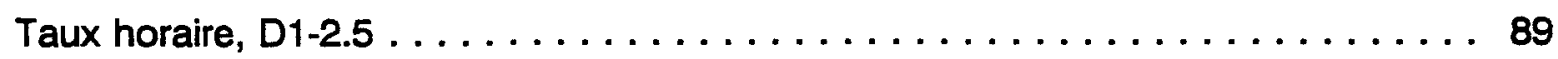

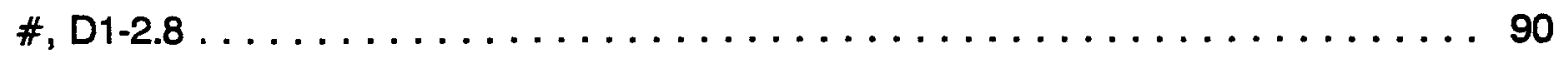

Écritures de journal, $2.5-\mathrm{DB} \ldots \ldots \ldots \ldots \ldots \ldots \ldots \ldots \ldots \ldots \ldots \ldots \ldots \ldots \ldots \ldots$

\# écritures, $\mathrm{D9}-2.5 \ldots \ldots \ldots \ldots \ldots \ldots \ldots \ldots \ldots \ldots \ldots \ldots \ldots \ldots \ldots \ldots \ldots \ldots$

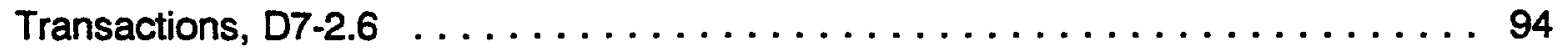

\section{Processus 2.1, Produire les feuilles de} temps

Diagramme de flux d'information $\ldots \ldots \ldots \ldots \ldots \ldots \ldots \ldots . . . \ldots 7$

2.1 Produire les feuille de temps $\ldots \ldots \ldots \ldots \ldots \ldots \ldots \ldots \ldots \ldots \ldots$

Processus de traitement $\ldots \ldots \ldots \ldots \ldots \ldots \ldots \ldots \ldots \ldots, 99$

Enregistrer données feuilles-temps, $2.1 .1 \ldots \ldots \ldots \ldots \ldots \ldots \ldots \ldots \ldots$

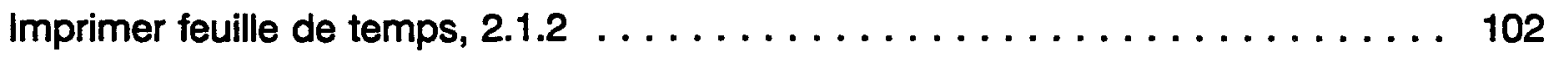

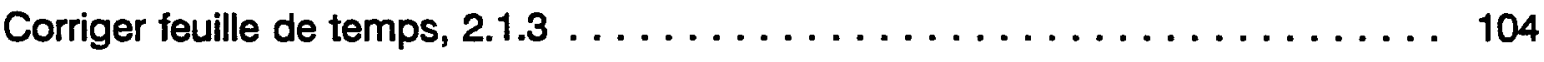

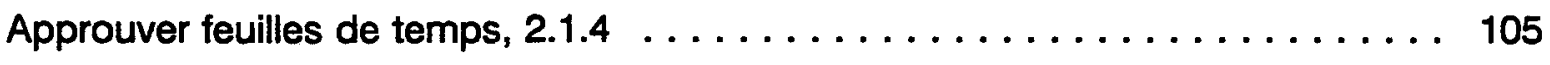

Flux de données ......................... 106

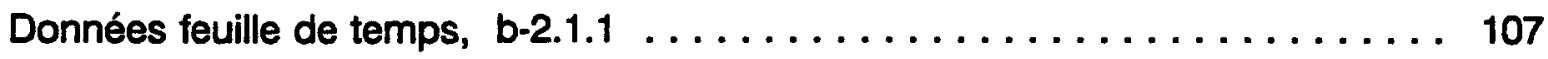

Date feuille de temps, b-2.1.1 ....................... 108

Nom et numéro, b-2.1.1 ........................... 109

Nom du client, 2.1.2-D2.1/2 $\ldots \ldots \ldots \ldots \ldots \ldots \ldots \ldots \ldots \ldots \ldots \ldots \ldots . \ldots \ldots$

Identification, 2.1.1-D2.1/2 $\ldots \ldots \ldots \ldots \ldots \ldots \ldots \ldots \ldots \ldots \ldots \ldots \ldots .112$

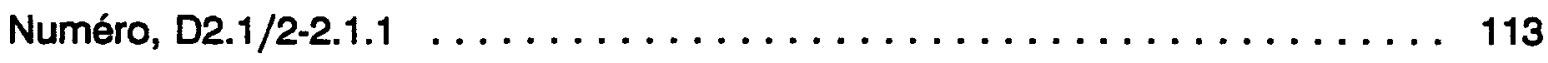

Nom, D2.1/2-2.1.1 ............................. 114

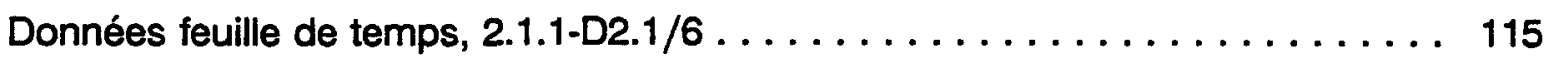

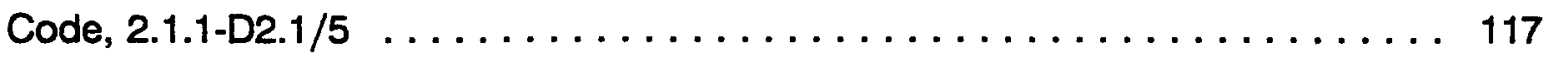

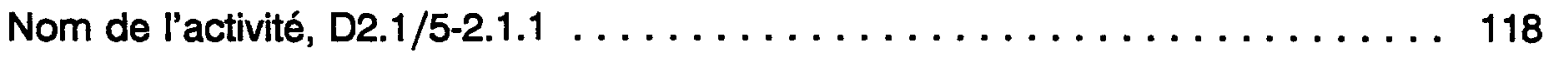

Coordonnées de la feuille de temps, $\mathrm{D} 2.1 / 3-2.1 .1 \ldots \ldots \ldots \ldots \ldots \ldots \ldots 119$ 
Date de la feuille de temps, $2.1 .1-D 2.1 / 3 \ldots \ldots \ldots \ldots \ldots \ldots \ldots \ldots \ldots 121$

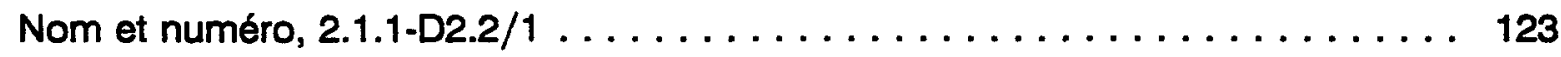

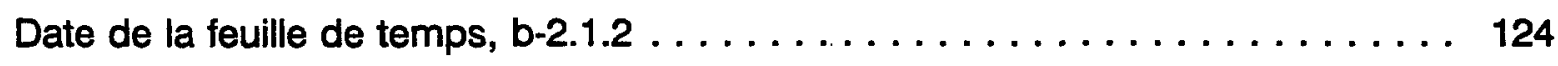

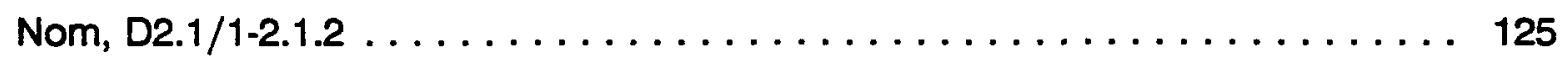

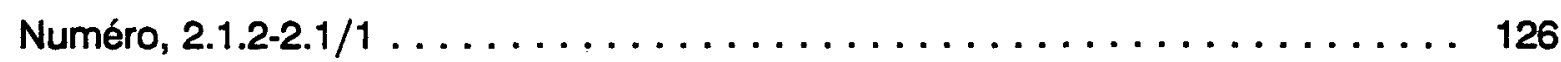

Date de la feuille de temps, $2.1 .2-\mathrm{D} 2.1 / 3 \ldots \ldots \ldots \ldots \ldots \ldots \ldots \ldots \ldots 127$

Coordonnées de la feuille de temps, D2.1/3-2.1.2 $\ldots \ldots \ldots \ldots \ldots \ldots \ldots 128$

Feuille de temps, $2.1 .2-2.1 .3 \ldots \ldots \ldots \ldots \ldots \ldots \ldots \ldots \ldots \ldots \ldots \ldots \ldots$

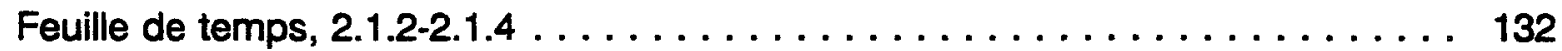

Transactions, $02.1 / 6-2.1 .2 \ldots \ldots \ldots \ldots \ldots \ldots \ldots \ldots \ldots \ldots \ldots \ldots \ldots . \ldots \ldots$

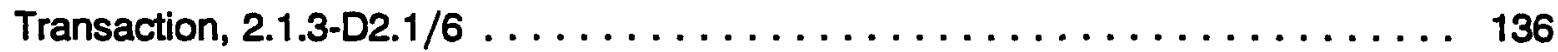

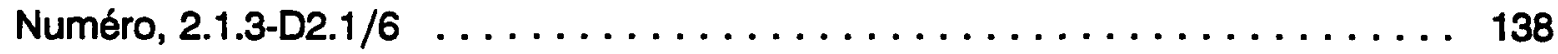

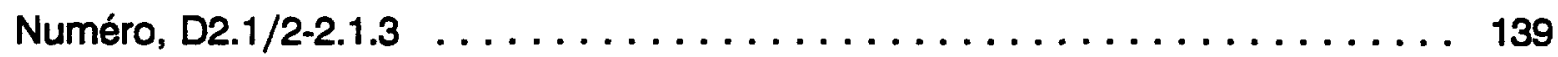

Nom du client, D2.1/2-2.1.3 . . . . . . . . . . . . . . . . 140

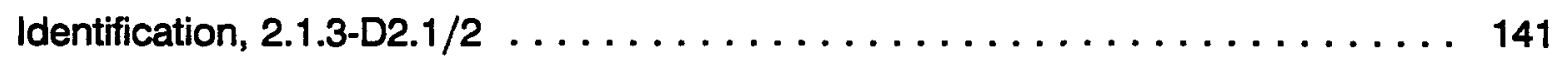

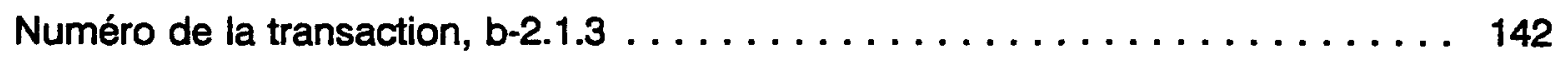

Nom de l'activité, $\mathrm{D} 2.1 / 5-2.1 .3 \ldots \ldots \ldots \ldots \ldots \ldots \ldots \ldots \ldots \ldots \ldots$

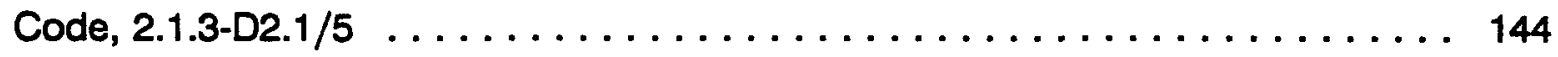

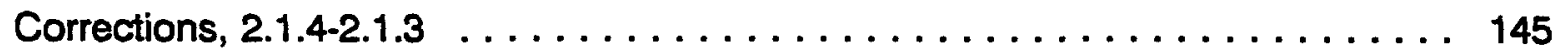

Feuilles de temps, $2.1 .4-\mathrm{D} 2.1 / 4 \ldots \ldots \ldots \ldots \ldots \ldots \ldots \ldots \ldots \ldots \ldots \ldots$

\# ass, 2.1.1-D2.1/2 ......................... 148

\# ass, $D 2.1 / 2-2.1 .3 \ldots \ldots \ldots \ldots \ldots \ldots \ldots \ldots \ldots \ldots \ldots \ldots \ldots \ldots . \ldots \ldots$

\section{Processus 2.6, Produire rapports} statistiques

Processus 2.7, Contrôler feuilles de

temps 
Diagramme de flux de données ................. 166

2.7 Contrôler feuilles de temps $\ldots \ldots \ldots \ldots \ldots \ldots \ldots \ldots$

Processus de traitement $\ldots \ldots \ldots \ldots \ldots \ldots \ldots \ldots \ldots \ldots, 168$

Déterminer feuilles de temps manquantes, $2.7 .1 \ldots \ldots \ldots \ldots$

Vérifier feuilles de temps manquantes, $2.7 .2 \ldots \ldots \ldots \ldots \ldots \ldots 17$

Calculer temps supplémentaire, $2.7 .3 \ldots \ldots \ldots \ldots \ldots$. . . . . . . . . 172

Flux de données ................................ 173

Date de la feuille de temps, $a-2.7 .2 \ldots \ldots \ldots \ldots \ldots \ldots \ldots \ldots$

Numéro d'associé, a-2.7.2 . . . . . . . . . . . . . . . . . . . . . 175

Feuilles de temps manquantes, $2.7 .2-a \ldots \ldots \ldots \ldots \ldots \ldots \ldots$

Coordonnées, D2/3-2.7.1 . . . . . . . . . . . . . . . . . . . . . . . 177

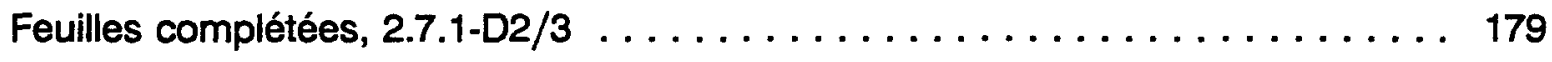

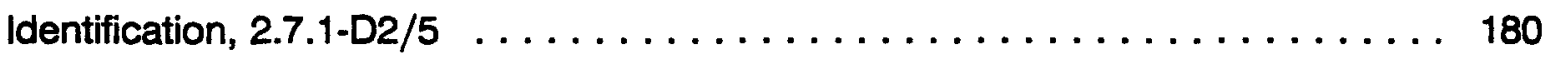

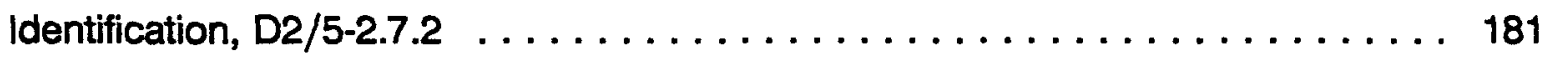

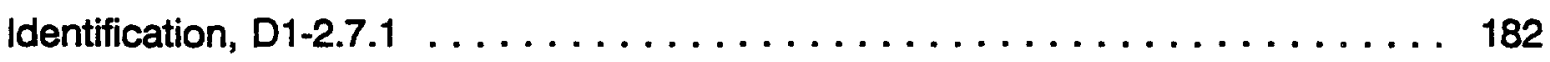

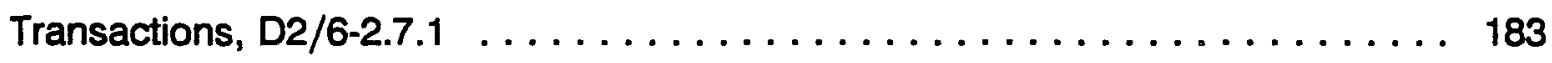

Transactions, $\mathrm{D} 2 / 6-2.7 .3 \ldots \ldots \ldots \ldots \ldots \ldots \ldots$

Coordonnées, $\mathrm{D} 2 / 3-2.7 .3 \ldots \ldots \ldots \ldots \ldots \ldots \ldots \ldots$

Temps supplémentaire, $2.7 .3-D 10 \ldots \ldots \ldots \ldots$. . . . . . . . . . . . . 188

Processus 5.2, Facturer les clients ......... 189

Diagramme de flux de données ................. 190

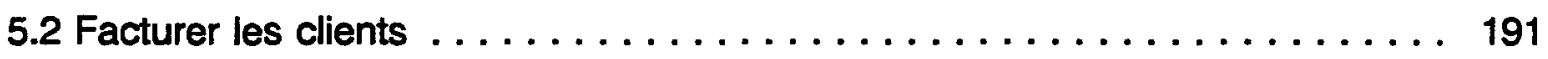

Processus de traitement $\ldots \ldots \ldots \ldots \ldots \ldots \ldots \ldots \ldots \ldots \ldots, 192$

Produire les factures, $5.2 .1 \ldots \ldots \ldots \ldots \ldots \ldots \ldots \ldots \ldots$

Dactylographier la facture, $5.2 .2 \ldots \ldots \ldots \ldots \ldots \ldots$. . . . . . . . . . . . 194

Saisir la facture, $5.2 .3 \ldots \ldots \ldots \ldots \ldots$. . . . . . . . . . . . . . . . 195 
Enregistrer la vente, $5.2 .4 \ldots \ldots \ldots \ldots \ldots \ldots \ldots \ldots \ldots \ldots \ldots . \ldots \ldots$

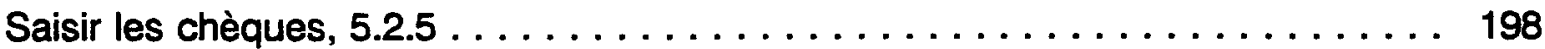

Enregistrer les recettes, $5.2 .6 \ldots \ldots \ldots \ldots \ldots \ldots \ldots \ldots \ldots \ldots \ldots \ldots . \ldots 199$

Flux de données ............................. 200

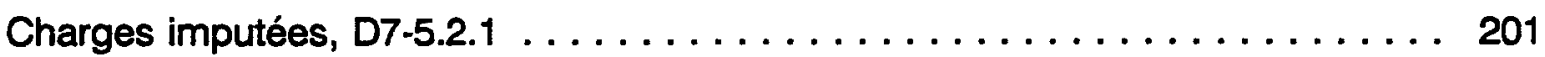

Nom, D2-5.2.1 . . . . . . . . . . . . . . . . . . . . . . . . . . 204

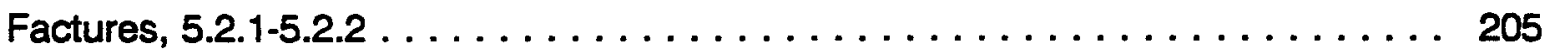

Factures, $5.2 .2-g \ldots \ldots \ldots \ldots \ldots \ldots \ldots \ldots \ldots \ldots \ldots \ldots \ldots \ldots .207$

Copie de la facture, $5.2 .2-D 5.2 / 1 \ldots \ldots \ldots \ldots \ldots \ldots \ldots \ldots \ldots . \ldots . \ldots 209$

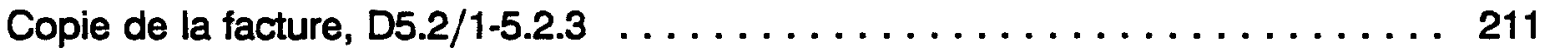

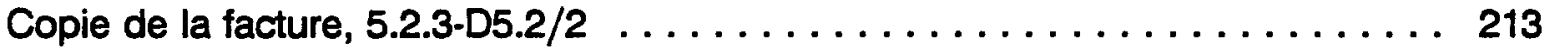

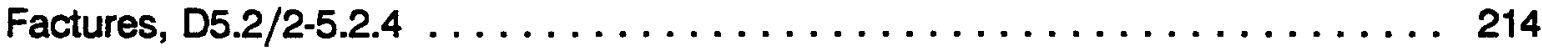

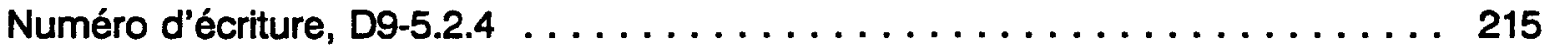

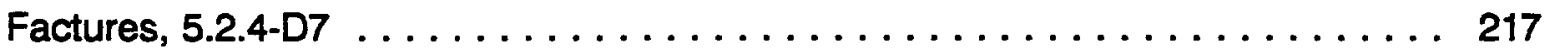

Factures, 5.2.4-D11 ........................... 219

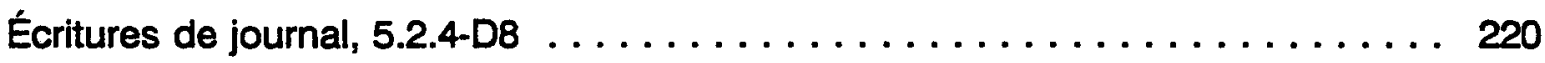

Numéro, D2-5.2.5 . . . . . . . . . . . . . . . . . . . . . 222

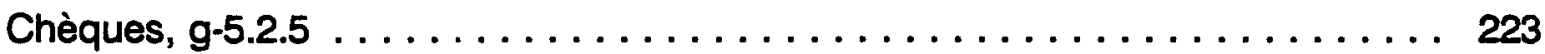

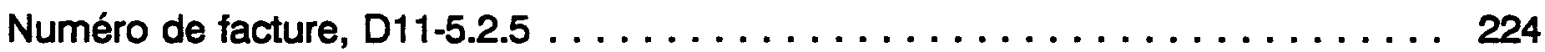

Numéro d'écritures, D9-5.2.6 ........................ 225

Recettes, $5.2 .5-D 5.2 / 4 \ldots \ldots \ldots \ldots \ldots \ldots \ldots \ldots \ldots \ldots \ldots \ldots \ldots .227$

Recettes, D5.2/4-5.2.6 .......................... 228

Information sur paiement, $5.2 .6-\mathrm{D} 11 \ldots \ldots \ldots \ldots \ldots \ldots \ldots \ldots \ldots \ldots 229$

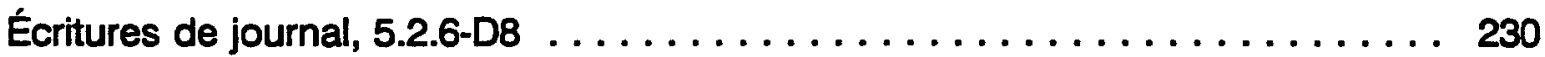

\section{Processus 5.5, Payer les fournisseurs .... 232}

Diagramme de flux de données $\ldots \ldots \ldots \ldots \ldots \ldots \ldots \ldots . \ldots 233$

5.5 Payer les fournisseurs $\ldots \ldots \ldots \ldots \ldots \ldots \ldots \ldots \ldots \ldots \ldots \ldots \ldots \ldots$ 
Processus de traitement $\ldots \ldots \ldots \ldots \ldots \ldots \ldots \ldots \ldots \ldots, 235$

Autoriser les factures, $5.5 .1 \ldots \ldots \ldots \ldots \ldots \ldots \ldots \ldots$

Inscrire les codes d'imputation, $5.5 .2 \ldots \ldots \ldots \ldots \ldots \ldots \ldots \ldots \ldots \ldots \ldots$

Saisir les factures, $5.5 .3 \ldots \ldots \ldots \ldots \ldots \ldots \ldots \ldots \ldots \ldots$

Imprimer liste des factures à payer, $5.5 .4 \ldots \ldots \ldots \ldots \ldots$. . . . . . . . . 240

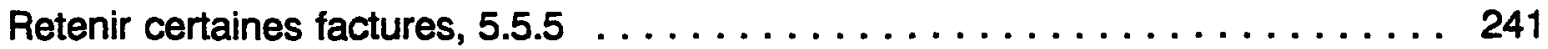

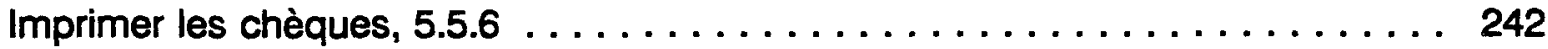

Imputer les dépenses, $5.5 .7 \ldots \ldots \ldots \ldots \ldots \ldots \ldots \ldots \ldots \ldots \ldots \ldots \ldots$

Enregistrer les nouveaux fournisseurs, $5.5 .8 \ldots \ldots \ldots \ldots \ldots \ldots$

Flux de données ................................ 248

Factures, d-5.5.1 . . . . . . . . . . . . . . . . . . . . . 249

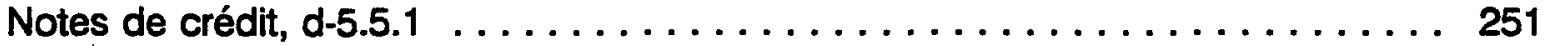

Autorisation, e-5.5.1 . . . . . . . . . . . . . . . . . . . 252

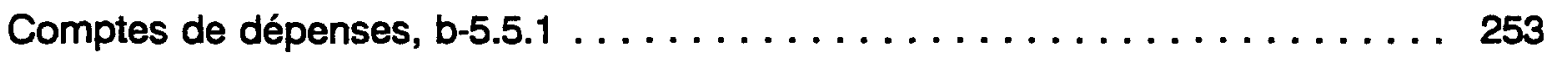

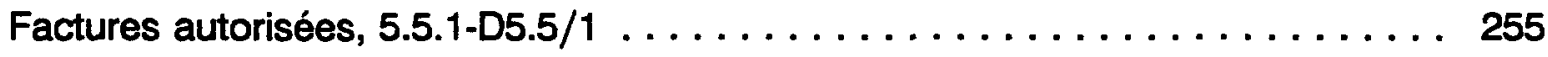

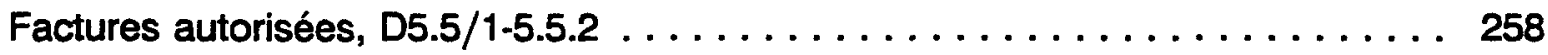

Numéro de compte, D3-5.5.2 . . . . . . . . . . . . . . . . . . . 260

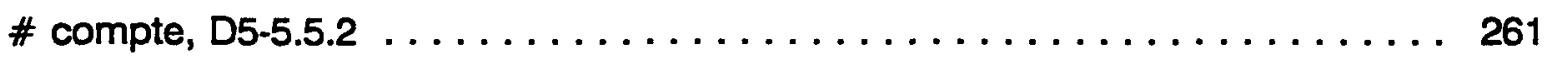

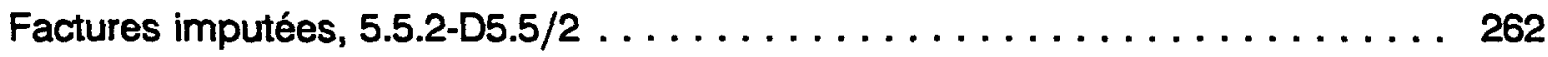

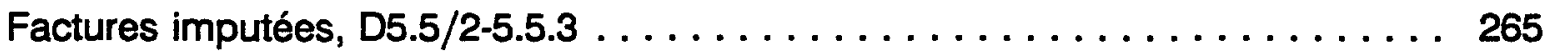

Nom du fournisseur, D5-5.5.3 . . . . . . . . . . . . . . . . . . . . . . . . . 267

Description du compte, D3-5.5.3 . . . . . . . . . . . . . . . . . . 268

Numéro du client, D2-5.5.3 . . . . . . . . . . . . . . . . . . . . . . . . . . . . . . 269

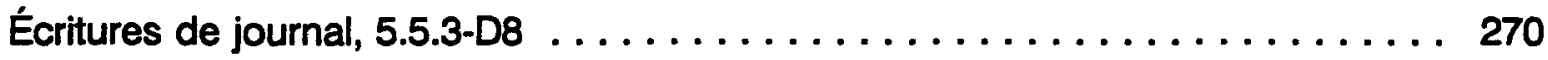

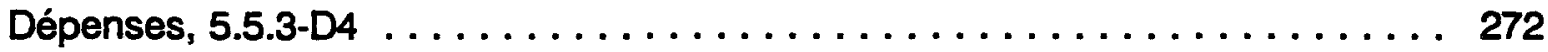

Numéro d'écritures, $\mathrm{D} 9-5.5 .3 \ldots \ldots \ldots \ldots \ldots \ldots \ldots \ldots \ldots$

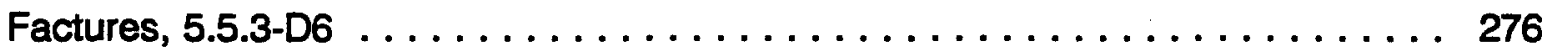

Dépenses, $04-5.5 .7 \ldots \ldots \ldots \ldots \ldots \ldots \ldots \ldots \ldots \ldots \ldots$

Écritures de journal, $5.5 .7-D 8 \ldots \ldots \ldots \ldots \ldots \ldots \ldots$

Dépenses facturables, $5.5 .7-D 7 \ldots \ldots \ldots \ldots \ldots \ldots \ldots$

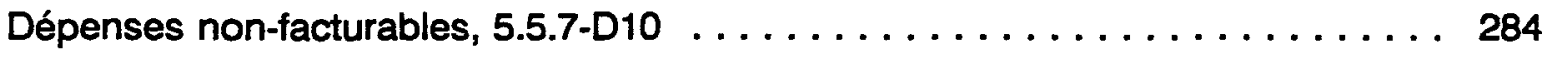

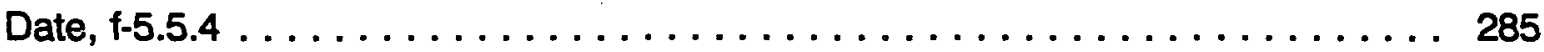




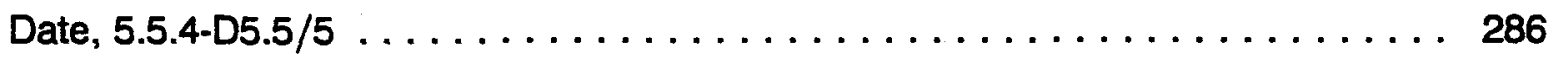

Liste des factures à payer, $5.5 .4-5.5 .5 \ldots \ldots \ldots \ldots \ldots \ldots \ldots \ldots \ldots \ldots 287$

Factures, D6-5.5.4 ............................ 289

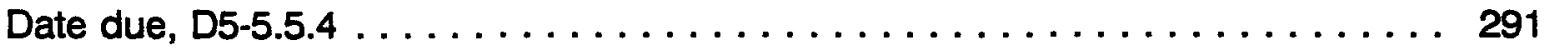

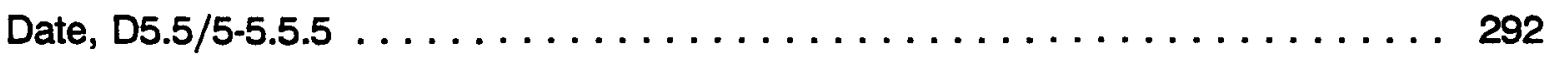

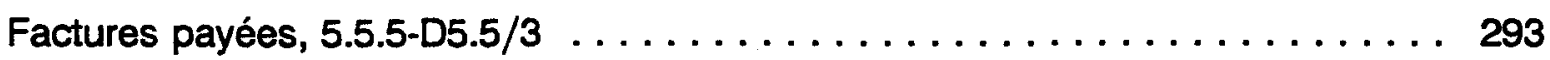

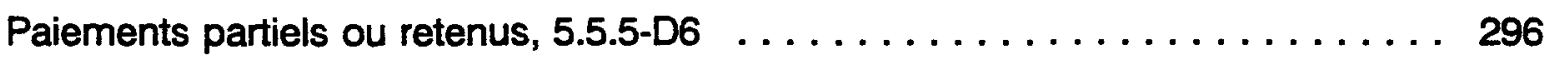

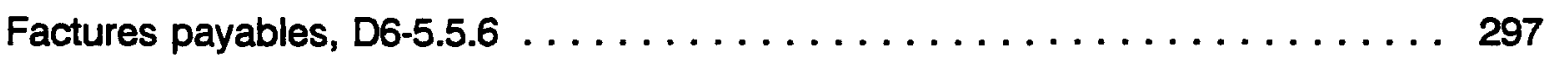

Montants, D5.5/6-5.5.6 . . . . . . . . . . . . . . . . . . . . . . . 299

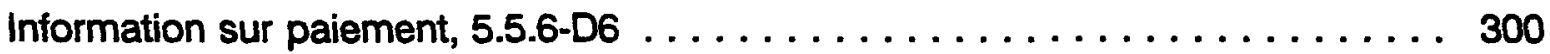

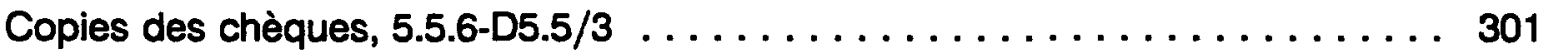

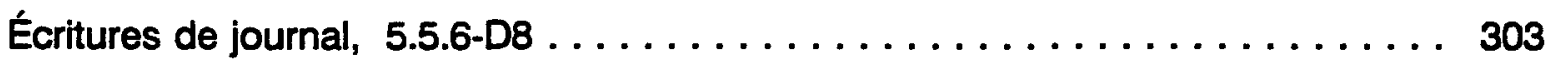

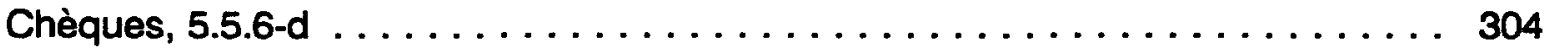

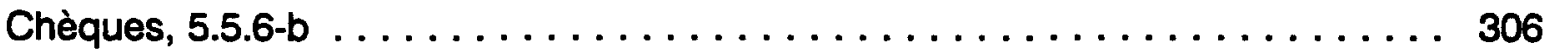

Coordonnées, D5-5.5.6 ........................ 308

Nom du fournisseur, D5-5.5.4 .................... 309

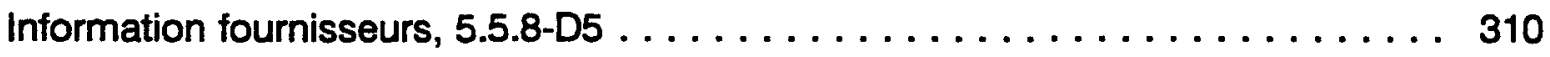

\section{Processus 5.6, Produire les états}

financiers

Diagramme de flux de données .................. 312

5.6 Produire les états financiers $\ldots \ldots \ldots \ldots \ldots \ldots \ldots \ldots \ldots \ldots \ldots \ldots \ldots \ldots$

Processus de traitement ...................... 314

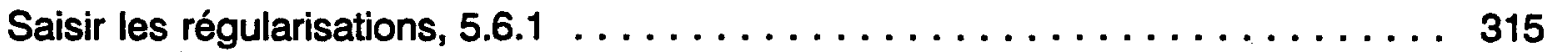

Enregistrer les écritures automatiques, $5.6 .2 \ldots \ldots \ldots \ldots \ldots \ldots \ldots \ldots \ldots 317$

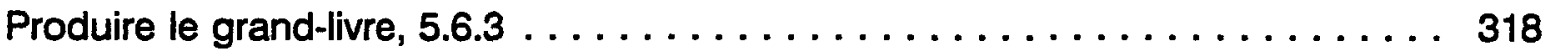

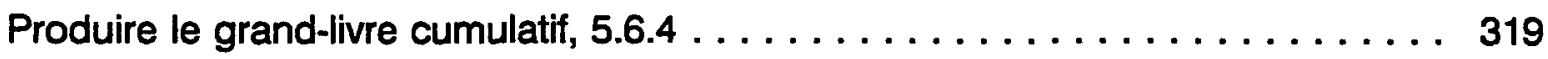

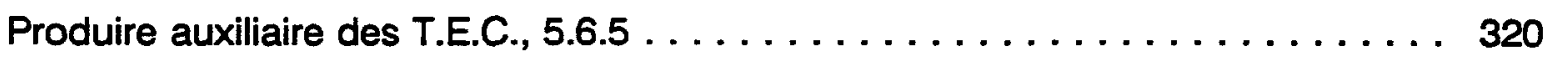


Produire l'auxiliaire des C.A.R., $5.5 .6 \ldots \ldots \ldots \ldots \ldots \ldots \ldots \ldots \ldots \ldots 22$

Produire l'auxiliaire comptes à payer, $5.6 .7 \ldots \ldots \ldots \ldots \ldots \ldots \ldots \ldots \ldots \ldots \ldots$

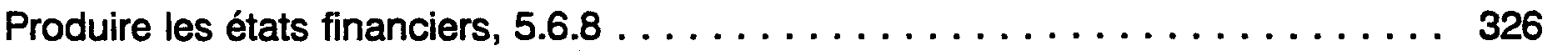

Flux de données $\ldots \ldots \ldots \ldots \ldots \ldots \ldots \ldots \ldots \ldots \ldots \ldots \ldots$

Régularisations, g-5.6.1 ....................... 329

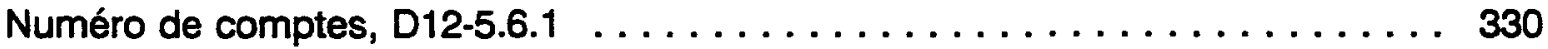

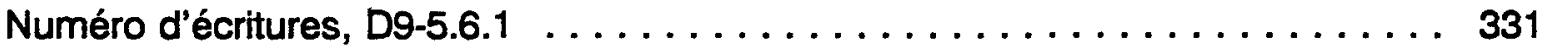

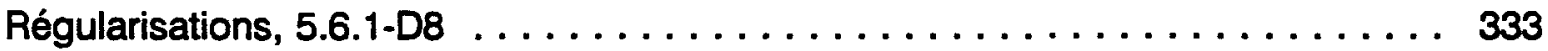

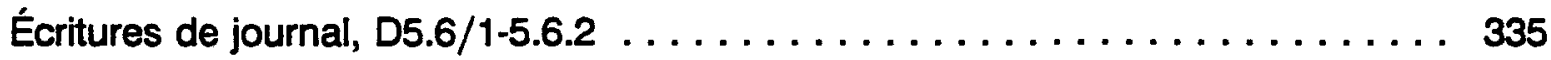

Écritures de journal, $5.6 .2-D 8 \ldots \ldots \ldots \ldots \ldots \ldots \ldots \ldots \ldots \ldots \ldots \ldots \ldots \ldots \ldots$

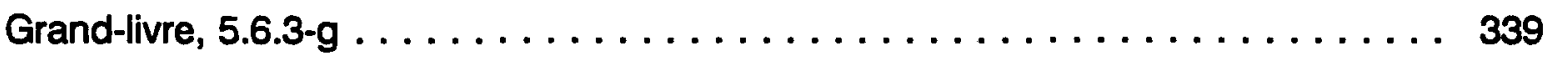

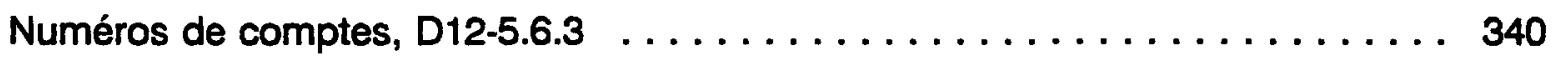

Nom des comptes, D12-5.6.3 ..................... 341

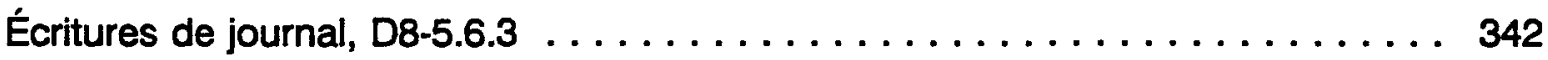

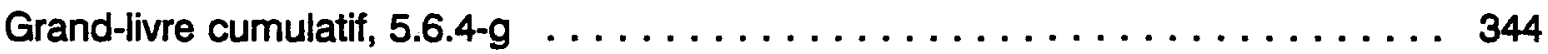

Nom des comptes, $D 12-5.6 .4 \ldots \ldots \ldots \ldots \ldots \ldots \ldots \ldots \ldots \ldots \ldots . \ldots \ldots$

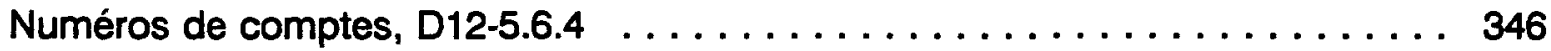

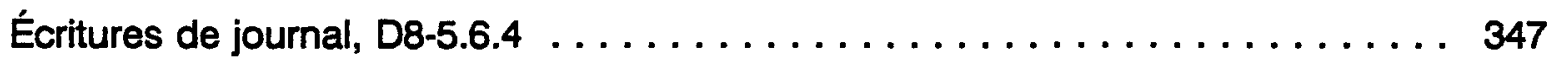

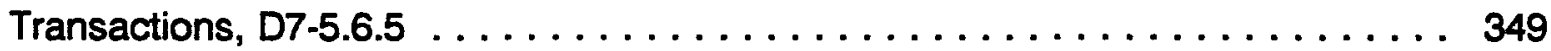

Auxiliaire des travaux en cours, $5.6 .5-\mathrm{g} \ldots \ldots \ldots \ldots \ldots \ldots \ldots \ldots \ldots \ldots 35$

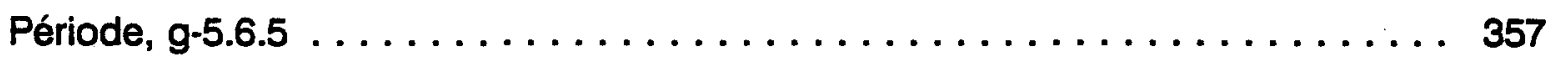

Numéros de clients, $D 2-5.6 .5 \ldots \ldots \ldots \ldots \ldots \ldots \ldots \ldots \ldots \ldots \ldots \ldots \ldots \ldots$

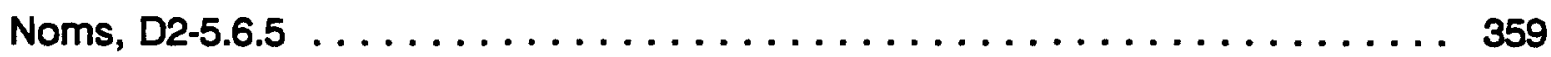

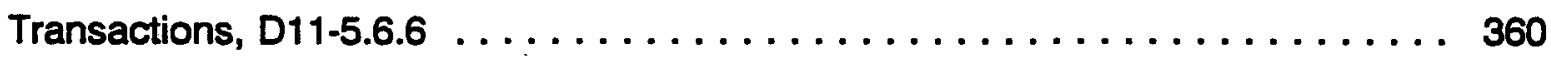

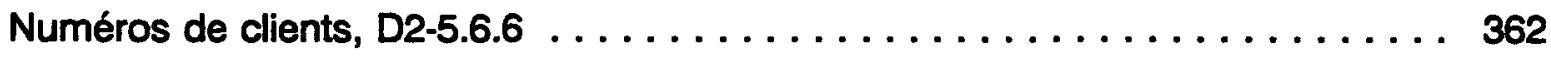

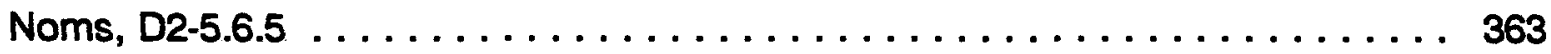

Auxiliaire des comptes a recevoir, $5.6 .5-g \ldots \ldots \ldots \ldots \ldots \ldots \ldots \ldots . \ldots 364$

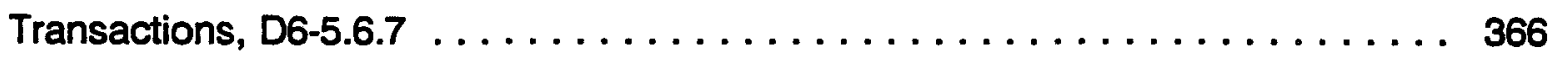

Période, g-5.6.7 ............................. 368

Auxiliaire des comptes à payer, $5.6 .7-\mathrm{g} \ldots \ldots \ldots \ldots \ldots \ldots \ldots \ldots \ldots \ldots . \ldots \ldots 96$

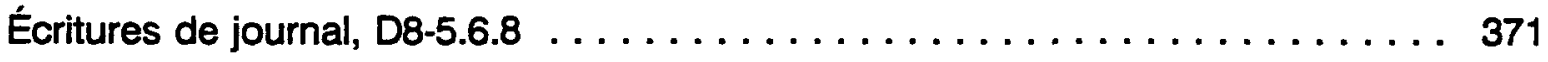

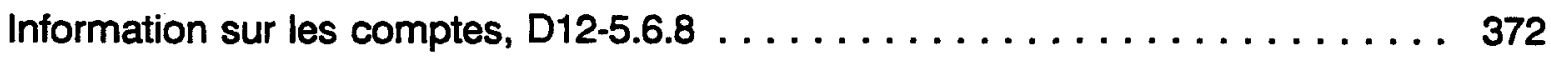

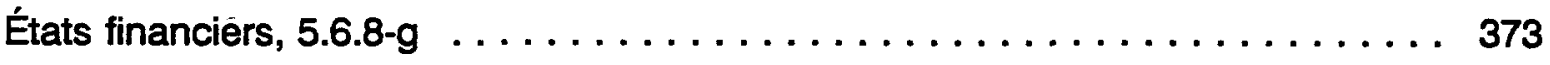


Index 
Processus 2.0, Compiler feuille de temps 
Diagramme de flux d'information 
2.0 Compiler le temps

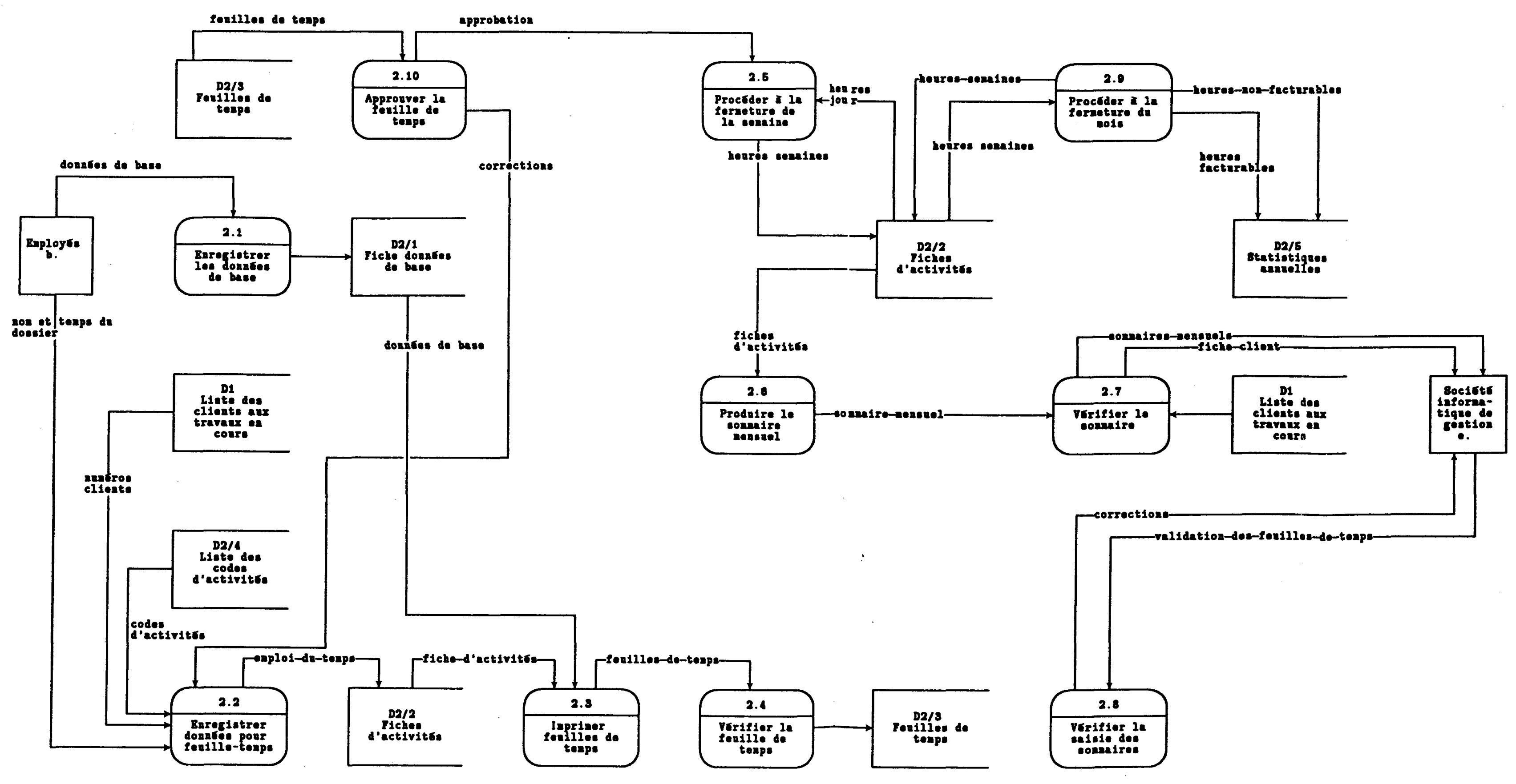




\section{Processus de traitement}


Processus de traitement

\section{Produire les feuilles de temps, 2.1}

\section{DESCRIPTION}

Ce processus regroupe les activités suivantes:

- enregistrer les données de la feuille de temps 2.1.1

- imprimer la feuille de temps 2.1.2

- corriger la feuille de temps 2.1.3

- approuver la feuille de temps 2.1 .4

Il est exécuté par tous les employés et associés de Harel, Drouin et associés, Harel Drouin Gestion Conseil et S.I.G.

\section{ENTRÉE}

SORTIE

Données feuille de temps b-2.1.1

\section{RÉSUMÉ DE LA LOGIQUE}

Enregistrer la feuille de temps Imprimer la feuille de temps

Corriger la feuille de temps Approuver la feuille de temps 
Processus de traitement

\section{Saisir données feuilles de temps, 2.8}

\section{DESCRIPTION}

Étant donné que la très grande majorité des feuilles de temps sont produites à l'aide de l'informatique, la saisie de ces feuilles consistera à récupérer les données qui sont sur les disquettes des individus. Par contre le système devra être suffisamment souple pour nous permettre la saisie de feuilles de temps produites manuellement.

Afin de maximiser l'utilisation de l'informatique et d'éviter que soit exécuté plusieurs fois le programme de validation des transactions, le système devra nous permettre d'accumuler un lot de transactions dans un fichier temporaire (exemple: toutes les personnes d'une même équipe) avant de les traiter.

\section{ENTRÉE}

transactions, b-2.8

feuilles de temps, b-2.8

date, $a-2.8$

dates, D2/3-2.8

nom du client, D2-2.8

\#, D1-2.8
SORTIE

transactions, 2.8-D2/6 


\section{RÉSUMÉ DE LA LOGIQUE}

demander la date pour laquelle on désire enregistrer des transactions valider la date fournie avec le fichier des coordonnées de feuilles de temps

lire dans le fichier des coordonnées de feuilles de temps (D2/6) les dates des jours compris dans la feuilles de temps

demander si la saisie des transactions est effectuée par disquettes ou par feuilles de temps

si la saisie est effectuée à partir de feuilles de temps

Enregistrez le numéro de l'employé ou associé

Validez le numéro de l'employé ou associé saisi avec ceux inscrit dans le fichier maître employés associés

Pour chaque transaction

Enregistrer la date de la transaction

Valider la date de la transaction avec les coordonnées de la feuille de temps

Demander si l'activité est facturable

Si l'activité est facturable

Enregistrer le numéro du client

Vérifier dans le fichier client si le client existe

si oui afficher le nom du client

sinon,

effacer le numéro du client déjà saisi ${ }^{(1)}$

Enregistrer le code d'activité

demander le nom du client et le numéro de l'associé

Vérifier si le code d'activité existe

Si le code existe

Si oui, affichez le nom de l'activité

Demander le temps passé à exécuter l'activité

Vérifiez si le temps est un multiple de .25

sinon, affichez message d'erreur

Enregistrer la description du travail effectué

Si l'activité n'est pas facturable

Enregistrer le code d'activité

Vérifier si le code d'activité existe

Si le code existe

Si oui, affichez le nom de l'activité

Demandez le temps passé à exécutez l'activité

Vérifiez si le temps est un multiple de .25

Sinon, affichez message d'erreur

Vérifiez si le code d'activité signifie NON à L'EMPLOI

Si oui vérifiez que le temps de l'activité ne soit pas supérieur à 7 heures.

Enregistrer la description du travail effectué

Mettre le code de validation égal à 2 (transaction non validée) 
si la saisie est effectué par disquettes

pour chaque disquette

lire toutes les transactions de la disquette et les mettre dans un fichier temporaire

pour toutes les transactions contenu dans le fichier mentionné précédemment

valider le numéro de l'employé ou associé avec ceux inscrits dans le fichier maître employés-associés

valider la date de la transaction avec les dates des journées comprises dans la semaine de feuille de temps

valider le code de l'activité avec ceux inscrits dans le fichier des codes d'activités

dans le cas d'activités facturables:

valider le numéro et le nom du client fournit avec ceux inscrits dans le fichier maitre clients

valider le temps de l'activité (multiple de 0.25)

saisir la description du travail effectué

dans le cas d'activités non facturables:

valider le temps de l'activité

saisir la description du travail effectué

Si un des champs d'une transaction n'est pas validé, mettre le code de validation égal à 0 . 
Processus de traitement

\section{Valider feuilles de temps, 2.2}

\section{DESCRIPTION}

Ce processus sera exécuté automatiquement par le système. II sera effectué à chaque fois que des transactions seront saisies (processus 2.8) et à chaque fois qu'il y aura des corrections de transactions.

La validation des feuilles de temps consiste à vérifier l'exactitude des transactions enregistrées. Cette validation produit un rapport dans lequel on retrouve toutes les transactions pour lesquelles il n'y a pas de numéro de client, les transactions dont le nom du client de correspond pas à celui figurant dans le fichier maître clients, celles dont le numéro d'employé ou d'associé est erroné, celles dont le code d'activité est erroné, celles qui ont un code d'activité $=000$ etc.. La validation des transactions attribue un code à la transaction qui signifie que la transaction a été validée et acceptée. Toutes les transactions qui apparaitront dans le rapport de validation seront identifiées comme étant non acceptées (à l'exception de celles dont le code d'activité $=000$ ).

\section{ENTRÉE}

numéro associés, a-2.2

transactions, D2/6-2.2
SORTIE

transactions, 2.2-D2/6

transactions erronées, 2.2-D2/1 


\section{RÉSUMÉ DE LA LOGIQUE}

Pour toutes les transactions contenues dans le fichier transactions

Lire une transaction

Regardez si code de validation $=0$

si oul,

inscrire la transaction dans le rapport de validation sinon

Regardez si code d'activité $=000$

si oui,

inscrire la transaction dans le rapport de validation

si oui, Regardez si la transaction a un numéro de client

regarder si le nom du client inscrit dans la transaction est le même que celui qui est inscrit dans le fichier maître clients

si oui, inscrire le code signifiant que la transaction est acceptée sinon, inscrire la transaction dans le rapport de validation

sinon, inscrire le code qui signifie que la transaction n'est pas acceptée

inscrire la transaction dans le rapport de validation inscrire le code qui signifie que la transaction n'est pas acceptée 
Processus de traitement

\section{Corriger les transactions, 2.3}

\section{DESCRIPTION}

Processus manuel qui permettra la modification de tous les champs d'une transaction. La sélection de la transaction sera effectuée en fournissant le numéro de la transaction. Si une transaction qui n'était pas dans le rapport de validation (D2/1) est modifiée, cette dernière devra être identifiée comme étant non validée. Elle ne pourra pas être utilisée pour la mise à jour des travaux en cours tant que celle-ci n'aura pas été validée à nouveau (processus 2.2).

Dans les cas d'enregistrements des nouveaux clients, le système devra demander le nom du client et le numéro de l'associé. Les transactions devront être affichées dans l'ordre numériques et le système devra permettre la sélection des transactions concernées par le numéro de client généré par le système en déplaçant le curseur à l'écran.

Ce processus sera exécuté que par certaines personnes dûment autorisées. Le système devra permettre la création automatique des nouveaux clients (processus 2.4) dans le fichier maître client.

ENTRÉES

corrections, a-2.3

nom, D2/2-2.3

Transactions D2/6-2.3
SORTIE

informations client, 2.3-2.4

Transactions 2.3-D2/6

code, 2.3-D5 
RÉSUMÉ DE LA LOGIQUE

Processus à accès restreint.

Vérifiez si l'usager est autorisé à utiliser ce processus.

Demandez le type d'opération désirée.

1) saisie de nouveaux clients

2) modification d'une transaction

1) saisie des noms de nouveaux clients

Triez les transactions dans l'ordre numérique

Demander le nom du client

Demander le numéro de l'associé

CRÉER UN NOUVEAU CLIENT (voir processus 2.4)

Affichez à l'écran toutes les transactions contenues dans le rapport de validation et mettre en évidence toutes les transactions n'ayant pas de numéro de client

Sélectionner à l'aide du curseur les transactions pour lesquelles on désire enregistrer le numéro de client assigné par le système, le nom du client, et le numéro de l'associé. Modifier le fichier des transactions feuilles de temps D2/6

Répéter l'opération

Retourner au menu

3) modification d'une transaction

Triez les transactions dans l'ordre alphabétique des noms et prénoms (pour un même personne, les transactions devront être numériques).

déplacer le curseur sur le champ à modifier

a) date de la transaction

si le curseur est sur la date de la transaction

Enregistrer la date de la transaction

Valider la date de la transaction avec les coordonnées de la feuille de temps 
b) code d'activité

si le curseur est sur le code de l'activité

Enregistrer le code d'activité

Vérifier si le code d'activité existe

Si le code existe

Si oui, affichez le nom de l'activité

sinon afficher message d'erreur et attendre nouveau code

Vérifiez si le code d'activité signifie NON A L'EMPLOI

Si oui vérifiez que le temps de l'activité ne soit pas supérieur à 7 heures.

Si le code signifie que l'activité est facturable

Vérifier s'il y a un numéro de client dans le champ de numéro de client, sinon déplacer le curseur sur le champ de numéro de client et attendre un numéro de client

Regarder dans le fichier maître client le nom du client correspondant au numéro fournit si client correspondant, afficher le nom du client

sinon demander nom du client

Demander le numéro de l'associé

Créer le nouveau client dans le fichier maître client (processus 2.4)

Si le code signifie que l'activité est non facturable

s'il y a un numéro de client dans le champ réservé à cet effet, effacer le numéro et le nom du client

c) le temps de l'activité

Si le curseur est sur le temps de l'activité

Demander le temps passé à exécuter l'activité

Vérifiez si le temps est un multiple de .25

sinon, affichez message d'erreur et attendre le temps

d) la description du travail effectué

si le curseur est sur description du travail effectué

Enregistrez la description du travail effectué

Indiquer que la transaction n',est pas validée

Le processus 2.3 doit être suivi du processus 2.2 automatiquement. 
Processus de traitement

\section{Créer un nouveau client, 2.4}

\section{DESCRIPTION}

Ce processus est exécuté automatiquement à l'intérieur du processus 2.3 Corriger les transactions lorsqu'il y a saisi de nom de nouveaux clients. Par contre le système devra permettre la création de nouveaux client dans le fichier maître client sans qu'il y ait des transactions pour lesquelles on désire enregistrer les noms des nouveaux clients.

Avec le nom du client saisi au processus 2.3, nous vérifions dans le fichier maître-clients si un client correspond au nom saisi. Si c'est le cas, le système mentionnera qu'il y a un client existant sous le même nom et demandera à l'usager s'il désire quand même créer le client. Si oui, le système assignera un numéro de client.

Dans les cas où aucun client du fichier maître ne correspond au nom saisi, le client sera créé dans le fichier maître client et le système assignera automatiquement un numéro au client saisi.

II faut prendre note que ce processus sera exécuté que par certaines personnes dûment autorisées.

ENTRÉES

information client, 2.3-2.4
SORTIE

information client, 2.4-D2

message, 2.4-a 


\section{RÉSUMÉ DE LA LOGIQUE}

Regardez dans le fichier client si le client existe

Si le client existe

si oui,

Affichez message mentionnant l'existence d'un client sous le même nom

Demandez si l'usager désire créer le client sous le même nom si oui, créer le client ${ }^{(1)}$

Affichez le nom du client inscrit dans le fichier maître sinon

Créez le client dans le fichier maître clients 
Processus de traitement

\section{Imputer le temps, 2.5}

\section{DESCRIPTION}

Pour chaque transaction de feuille de temps enregistrée dans le fichier Transactions feuilles de temps $\mathrm{D} 2 / 6$, ce processus consiste à traiter, pour un ou plusieurs associés, toutes les transactions qui contiennent un numéro d'associé, à inscrire les transactions facturables dans l'auxiliaire des travaux en cours.

Avant d'imputer les transactions, le système devra vérifier s'il y $a$, dans le fichier de rapport de validation, des transactions ayant le numéro d'un des associés pour lesquels on demande l'imputation du temps. S'il y a des transactions, le système devra en aviser l'usager et demander si nous devons quand même procéder à l'imputation. Le système devra également véritier s'il y a, dans le fichier "D2/5 feuilles de temps manquantes", des enregistrements contenant le ou les numéros d'associés fournis. Dans l'affirmative, ces enregistrements devront être affichés à l'écran.

Le processus sera effectué par les personnes dûment autorisées.

\section{ENTRÉE}

Transactions, D2/6-2.5

Numéro de l'associé A-2.5

Numéro de l'associé, D1-2.5

Taux horaire, D1-2.5

Rapport de validation, D2/1-2.5

\# écriture, D9-2.5
SORTIE

Transactions facturables 2.5-D7

Date et numéro de l'associé 2.5-D2/7

Écritures de journal, 2.5-D8 


\section{RÉSUMÉ DE LA LOGIQUE}

Mettre le cumulatif correspondant aux associés à zéro.

Demandez le numéro de l'associé ou des associés pour lesquels on désire imputer le temps Validez le numéro de l'associé si numéro inexistant, affichez message d'erreur

Regardez s'il y a, dans le fichier "D2/5 feuilles de temps manquantes", des enregistrements contenant le numéro de l'associé

si oui, affichez les enregistrements correspondants

Regardez dans le fichier "D2/1 Rapport de validation", s'il y a des transactions ayant le numéro de l'associé fourni

si oui, affichez message mentionnant qu'il a y a des transactions à corriger demandez si l'imputation doit être quand même être faite si oui, faire imputation

sinon, faire imputation.

faire imputation:

Pour toutes les transactions du fichier transactions feuilles de temps D2/6 qui ont été validées, dont le numéro de l'associé correspond à un des numéros d'associés fournis et dont le code d'imputation signifie que la transaction n'a pas été traitée ${ }^{(1)}$

Regardez si la transaction est facturable

si oui,

lire dans le fichier maître employés-associés, le taux horaire correspondant au numéro inscrit dans la transaction

multiplier le taux horaire par le temps de la transaction

Inscrire le résultat de la multiplication, le code de transaction, le numéro de l'écriture et le contenu de la transaction dans l'auxiliaire des travaux en cours.

additionner le montant de la transaction au cumulatif de l'écriture.

Modifier le code d'imputation pour qu'il signifie que la transaction a été traitée ${ }^{(2)}$ sinon,

Modifier le code de transaction pour qu'il signifie que la transaction a été traitée

1- imputation aux travaux en cours

2- imputation aux travaux en cours ou inscription dans le fichier cumulatif des transactions 
Lorsqu'il n'y a plus de transaction pour l'associé, ou les associés

inscrire dans le fichier des dates d'imputation, les numéros des associés et la date de la mise à jour des travaux en cours.

inscrire:

le numéro de l'écriture (assigné par le système)

le code de transaction (1)

la date de la transaction

le numéro de compte des travaux en cours

le cumulatif de l'écriture

inscrire:

le numéro de l'écriture

le code de transaction

la date de transaction

le numéro de comptes de revenus

le cumulatif de l'écriture

dans le fichier des écritures de journal.

1- code de transaction: (1) transaction au journal général

(2) transaction au joumal des revenus

(3) transaction au journal de la facturation

(4) transaction au caisse recettes

(5) transaction au caise déboursés

(6) transaction au journal des achats 
Processus de traitement

\section{Produire rapport statistique, 2.6}

\section{DESCRIPTION}

Ce processus consiste à imprimer un ensemble de rapports statistiques demandé par un usager. Pour connaître les rapports statistiques fournit par ce processus, voir la section 2.6 traitant des rapports statiques.

\section{ENTRÉE}

information pour rapport, c-2.6

transactions, D2/4-2.6
SORTIE

rapport statistique, 2.6-c 
Processus de traitement

\section{Contrôler feuilles de temps, 2.7}

\section{DESCRIPTION}

Ce processus regroupe trois activités:

déterminer les feuilles de temps manquantes 2.7.1

vérifier les feuilles de temps manquantes 2.7.2

calculer le temps supplémentaire 2.7 .3

et, est exécuté pour toutes les semaines de feuilles de temps non complétées excluant les semaines suivant la semaine courante. L'activité 2.7.1 déterminer feuilles de temps manquantes est transparente à l'utilisateur, c'est à dire qu'elle est automatiquement exécutée par le système. L'activité 2.7.2 Vérifier les feuilles de temps manquantes consiste à afficher sur l'écran, pour un associé, les noms des personnes pour lesquelles la feuilles de temps n'a pas été saisie. L'activité 2.7.3 consiste à calculer, pour chaque journée, le temps supplémentaire de chaque employé. Le temps supplémentaire est enregistré sous forme d'une transaction de feuille de temps dans le fichier "D10 Cumulatif des transactions". Le code d'activité utilisé nous permettra d'identifier, parmi toutes les transactions de feuille de temps non facturables, les transactions de temps supplémentaire. L'activité 2.3 inscrira toutes les transactions de feuilles de temps non facturables validées (les transactions facturables sont inscrites dans l'auxiliaire des travaux en cours), qui ont une des dates des journées comprises dans la semaine de feuilles de temps identifiée comme étant complétée, dans le fichier "D10 Cumulatif des transactions" et éliminera, du fichier "D2/6 Transactions feuilles de temps", toutes les transactions de feuilles de temps (facturables et non facturables) qui ont une des dates des journées comprises dans la semaine de feuilles de temps qui vient d'être identifiée comme étant complétée. 


\section{ENTRÉE}

Date de la feuille de temps, A-2.7

Numéro de l'associé, A-2.7

Identification, D1-2.7

identification, D2/5-2.7

Transactions, D2/6-2.7

Coordonnées, D2/3-2.7

\section{SORTIE}

Feuilles de temps manquantes, 2.7-A

Identification, 2.7-D2/5

Feuilles complétées, 2.7-D2/3

Temps supplémentaire, 2.7-D10

Transactions, 2.7-D10

\section{RÉSUMÉ DE LA LOGIQUE}

déterminer les feuilles de temps manquantes 2.7.1 vérifier les feuilles de temps manquantes 2.7.2 calculer le temps supplémentaire 2.7.3 
Flux de données 
Flux de données

\title{
Données de feuilles de temps, b-2.1
}

\author{
REF. SOURCE: b DESTINATION: Membre du personnel \\ $\begin{array}{llll}\text { REF. DESTIN.: } & 2.1 & \text { DESTINATION: } & \text { Produire feuilles de temps }\end{array}$
}

DESCRIPTION DÉTAILLÉE

Les données de la feuille de temps représentent toute l'information relative à l'emploi du temps d'un employé ou associé. A chaque jour, chaque membre du personnel ( Associés et employés) note dans leur agenda les tâches effectuées et le temps qui leurs est consacré.

\section{STRUCTURE DE DONNÉES INCLUSES}

Ce flux d'information regroupe les flux de données suivants:

- données feuilles de temps b-2.1.1

- date de la feuille de temps b-2.1.1

- date de la feuille de temps b-2.1.2

- numéro de la transaction b-2.1.3

- numéro et nom b-2.1.1

\section{VOLUME}

Harel Drouin, et Associés $\quad: \quad 35,280$

Harel Drouin Gestion Conseil $\quad$ : 5,880

Société d'informatique de gestion $: \underline{\mathbf{8 . 8 2 0}}$

Total par semaine $\quad: 49,980$ 
Flux de données

\section{Transactions, $b-2.8$}

$\begin{array}{llll}\text { REF. SOURCE: } & b & \text { DESCRIPTION: } & \text { Membre du personnel } \\ \text { REF. DESTIN.: } & 2.8 & \text { DESCRIPTION: } & \text { Saisir feuilles de temps }\end{array}$

\section{DESCRIPTION DÉTAILLÉE}

Contient l'information relative à l'emploi d'un employé ou d'un associé. Ces transactions proviennent des disquettes de feuilles de temps des employés ou de feuilles de temps.

\section{STRUCTURE DE DONNÉES INCLUSES}

numéro de l'employé ou de l'associé: 3 caractères numériques

date de la transaction: 8 caractères alphanumériques

numéro du client: 8 caractères numériques

nom du client: 60 caractères alphanumériques

numéro de l'associé ${ }^{(1)}: 3$ caractères numériques

code de l'activité: 3 caractères numériques

temps: 4 caractères numériques ( 2 décimales)

description de l'activité: $\mathbf{4 0}$ caractères alphanumériques

code de validation: 1 caractère numérique, $1=$ transaction acceptée, $0=$ transaction refusée code d'imputation: 1 caractère numérique, $1=$ transaction traitée, $0=$ transaction non traitée 
VOLUME

Harel Drouin, et Associés $\quad: 52,920$

Harel Drouin Gestion Conseil $\quad: \quad 8,820$

Société d'informatique de gestion : 13,230

Total par semaine $\quad: \mathbf{7 4 , 2 4 0}$

\section{HYPOTHĖSE}

4 transactions par heures

7 heures par jour

7 jours par semaines

180 employés

$50 \%$ des employé impriment 2 fois $\ldots>17.640$

TOTAL $\quad 52,920$ 
Flux de données

Feuilles de temps, b-2.8

$\begin{array}{llll}\text { REF. SOURCE: } & \text { b } & \text { DESTINATION: } & \text { Membre du personnel } \\ \text { REF. DESTIN.: } & 2.8 & \text { DESTINATION: } & \text { Saisir données feuilles de temps }\end{array}$

\section{DESCRIPTION DÉTAILLÉE}

Les feuilles de temps contiennent toute l'information relative à l'emploi du temps des employés ou associés. A chaque jour, chaque membre du personnel (Associés et employés) note dans leur agenda les tâches effectuées et le temps qui leurs est consacré.

A chaque semaine, chaque employé ou associé reporte son emploi du temps sur la feuille de temps.

STRUCTURE DE DONNES INCLUSES:

date du jour: 2 caractères numériques

nom du client: 60 caractères alphanumériques

code de l'activité: 3 caractères numériques

description de l'activité: 40 caractères alphanumériques

temps: 4 caractères numériques ( 2 décimales)

VOLUME

Harel Drouin, et Associés $\quad: 180$

Harel Drouin Gestion Conseil : $\quad 30$

Société d'informatique de gestion : 45

Total par semaine $\quad$ : 255 
Flux de données

Code, D2/2-2.8

$\begin{array}{llll}\text { REF. SOURCE: } & \text { D2/2 } & \text { DESCRIPTION: } & \text { Codes d'activités } \\ \text { REF. DESTIN.: } & 2.8 & \text { DESCRIPTION: } & \text { Saisir données feuilles de temps }\end{array}$

DESCRIPTION DÉTAILLÉE

Code de l'activité ( 3 chiffres ). Le code est seulement utilisé lorsqu'il y a saisie de données de feuille de temps directement à partir de feuilles de temps. Lorsqu'il y a saisie à partir de disquettes, le code n'a pas besoin d'être validé puisqu'il l'a été lors de la saisie de données de feuilles de temps (processus 2.1.1).

STRUCTURE DE DONNÉES INCLUSES

code de l'activité: 3 caractères numériques

VOLUME

Harel Drouin, et Associés $\quad: 10$

Harel Drouin Gestion Conseil : : 4

Société d'informatique de gestion : 4

Total par semaine $\quad: 18$ 
Flux de données

Date, a-2.8

$\begin{array}{llll}\text { REF. SOURCE: } & a & \text { DESCRIPTION: } & \text { Employés } \\ \text { REF. DESTIN.: } & 2.8 & \text { DESCRIPTION: } & \text { Saisir données feuilles de temps }\end{array}$

DESCRIPTION DÉTAILLÉE

Représente la date de la feuille de temps pour laquelle on désire saisir les transactions de feuilles de temps ou des feuilles de temps.

Dans le cas de saisie à partir de disquettes, cette date est utilisée pour sélectionner, parmi toutes les transactions lues à partir des disquettes, les transactions qui seront traitées. Dans le cas des feuilles de temps, elle permet de déterminer les journées pour lesquelles nous pouvons enregistrer des transactions.

STRUCTURE DE DONNÉES INCLUSES

date: 8 caractères alphanumériques

VOLUME

Harel Drouin, et Associés : $104^{(1)}$

Harel Drouin Gestion Conseil : 104

Société d'informatique de gestion : 104

Total par semaine $\quad: 312$

1. 52 semaines pour les feuilles de temps manuelles + 52 semaines pour la saisie à partir de disquettes 
Flux de données

\section{Nom client, D2-2.8}

$\begin{array}{llll}\text { REF. SOURCE: } & \text { D2 } & \text { DESCRIPTION: } & \text { Fichier maître client } \\ \text { REF. DESTIN.: } & 2.8 & \text { DESCRIPTION: } & \text { Saisir données feuilles de temps }\end{array}$

\section{DESCRIPTION DÉTAILLÉE}

Ce flux contient le nom du client. Il est employé pour afficher le nom du client correspondant au numéro de client saisi (saisie de données de feuilles de temps à partir de feuilles produites manuellement).

STRUCTURE DE DONNÉES INCLUSES

nom client: 60 caractères alphanumériques

VOLUME

Harel Drouin, et Associés $\quad: 35,280^{(1)}$

Harel Drouin Gestion Conseil : 5,880

Société d'informatique de gestion $: \underline{\mathbf{8 , 8 2 0}}$

Total par semaine $\quad: 49,980$ 
Flux de données

\section{Transactions, 2.8-D2/6}

$\begin{array}{llll}\text { REF. SOURCE: } & 2.8 & \text { DESCRIPTION: } & \text { Saisir données feuilles de temps } \\ \text { REF. DESTIN.: } & \text { D2/6 } & \text { DESCRIPTION: } & \text { Transactions feuilles de temps }\end{array}$

\section{DESCRIPTION DÉTAILLÉE}

Contient l'information relative à l'emploi du temps d'une personne pour un moment donné.

\section{STRUCTURE DE DONNÉES INCLUSE}

numéro de la transaction: 5 caractères numériques

numéro de l'employé ou de l'associé: 3 caractères numériques

date de la transaction: 8 caractères alphanumériques

numéro du client: 8 caractères numériques

nom du client: 60 caractères alphanumériques

numéro de l'associé ${ }^{(1)}: 3$ caractères numériques

code de l'activité: 3 caractères numériques

temps: 4 caractères numériques ( 2 décimales)

code de validation: 1 caractère numérique, $1=$ transaction acceptée, $0=$ transaction refusée code d'imputation: 1 caractère numérique, $1=$ transaction traitée, $0=$ transaction non traitée 
VOLUME

Harel Drouin, et Associés $\quad: 35,280$

Harel Drouin Gestion Conseil $\quad$ : 5,880

Société d'informatique de gestion $: \underline{\mathbf{8 . 8 2 0}}$

Total par semaine $\quad: 49,980$ 
Flux de données

\section{Dates, D2/3-2.8}

$\begin{array}{llll}\text { REF. SOURCE: } & \text { D2/3 } & \text { DESCRIPTION: } & \text { Coordonnées feuilles de temps } \\ \text { REF. DESTIN.: } & 2.8 & \text { DESCRIPTION: } & \text { Saisir feuilles de temps }\end{array}$

\section{DESCRIPTION DÉTAILLÉE}

Ce flux utilisé par les processus 2.8 Saisir les données feuilles de temps et sert à sélectionner les transactions à traiter (saisie à partir de disquettes) ou à valider les dates de transactions fournies par l'usager ( saisie à partir de feuilles de temps).

\section{STRUCTURE DE DONNÉES INCLUSES}

date de la feuille de temps: 8 caractères alphanumériques

1 ère journée: 8 caractères alphanumériques

nombre d'heures régulières pour la première journée: 1 caractère numérique 2 ème journée: 8 caractères alphanumériques

nombre d'heures régulières pour la deuxième journée: 1 caractère numérique 3 ème journée: 8 caractères alphanumériques nombre d'heures régulières pour la troisième journée: 1 caractère numérique 4 ème journée: 8 caractères alphanumériques nombre d'heures régulières pour la quatrième journée: 1 caractère numérique 5 ème journée: 8 caractères alphanumériques nombre d'heures régulières pour la cinquième journée: 1 caractère numérique 6 ème journée: 8 caractères alphanumériques nombre d'heures régulières pour la sixième journée: 1 caractère numérique 
7 ème journée: 8 caractères alphanumériques

nombre d'heures régulières pour la septième journée: 1 caractère numérique

VOLUME

Harel Drouin, et Associés $\quad: 180$

Harel Drouin Gestion Conseil : $\quad$ : 30

Société d'informatique de gestion : $\underline{45}$

Total par semaine $\quad$ : 255 
Flux de données

Numéro, 2.2-D2

REF. SOURCE:

DESCRIPTION:

Valider transactions feuilles de temps

REF. DESTIN.: $\quad$ D2

DESCRIPTION:

Fichier maître client

\section{DESCRIPTION}

Le numéro est utilisé pour sélectionner, dans le fichier D2 maître client, le client correspondant au numéro du client inscrit dans la transaction. Une fois que le client correspondant au numéro inscrit dans la transaction est identifié, nous comparons le nom du client inscrit dans le fichier maître avec le nom du client inscrit dans la transaction. Si les noms ne sont pas identiques, nous attribuons un code à la transaction qui signifie que la transaction n'est pas acceptée, la transaction sera inscrite dans le rapport de validation.

STRUCTURE DE DONNÉES INCLUSES

numéro: 8 caractères numériques

VOLUME

Harel Drouin, et Associés $\quad: 35,280$

Harel Drouin Gestion Conseil $\quad: 5,880$

Société d'informatique de gestion : $\underline{\mathbf{8 . 8 2 0}}$

Total par semaine $\quad: 49,980$ 
Flux de données

\section{Nom, D2-2.2}

$\begin{array}{llll}\text { REF. SOURCE: } & \text { D2 } & \text { DESCRIPTION: } & \text { Fichier maître client } \\ \text { REF. DESTIN.: } & 2.2 & \text { DESCRIPTION: } & \text { Valider transactions feuilles de } \\ & & & \text { temps }\end{array}$

DESCRIPTION

C'est le nom du client qui a été lu dans le fichier D2 maître client à l'aide du numéro de client (2.2-D2). Ce nom est comparé le nom du client inscrit dans la transaction. Si les noms ne sont pas identiques, nous attribuons un code à la transaction qui signifie que la transactions est n'est pas acceptée, la transaction sera inscrite dans le rapport de validation.

STRUCTURE DE DONNÉES INCLUSES

nom: 60 caractères alphanumériques

$\begin{array}{ll}\text { VOLUME } & \\ \text { Harel Drouin, et Associés } & : 35,280 \\ \text { Harel Drouin Gestion Conseil } & : 5,880 \\ \text { Société d'informatique de gestion } & : \underline{8,820} \\ \text { Total par semaine } & : 49,980\end{array}$


Flux de données

\section{Transactions, 2.2-D2/6}

REF. SOURCE: $2.2 \quad$ DESCRIPTION:

Valider Transactions feuillestemps

REF. DESTIN.: $\quad$ D2/6 DESCRIPTION: $\quad$ Transactions feuilles de temps DESCRIPTION DÉTAILLÉE

Contient toutes les transactions de feuilles de temps validées. Certaines de ces transactions ont le code de validation signifiant que la transaction a été acceptée, d'autres contiennent le code mentionnant que la transaction a été refusée.

\section{STRUCTURE DE DONNÉES INCLUSES}

numéro de la transaction: 5 caractères numériques

numéro de l'employé ou de l'associé: 3 caractères numériques

date de la transaction: 8 caractères alphanumériques

numéro du client: 8 caractères numériques

nom du client: 60 caractères alphanumériques

numéro de l'associé ${ }^{(1)}$ : 3 caractères numériques

code de l'activité: 3 caractères numériques

temps: 4 caractères numériques ( 2 décimales)

description de l'activité: 40 caractères alphanumériques

code de validation: 1 caractère numérique, 1 = transaction acceptée, $0=$ transaction refusée code d'imputation: 1 caractère numérique, 1 = transaction traitée, $0=$ transaction non traitée

1- dans le cas de transactions facturables le numéro de l'associé correspond au numéro de l'assocía auquel appartient le client, dans le cas de transactions non facturables le numéro de l'associé correspond au numéro de l'associé duquel relève l'employé. 
VOLUME

Harel Drouin, et Associés $\quad: 35,280$

Harel Drouin Gestion Conseil $\quad$ : 5,880

Société d'informatique de gestion $: \underline{\mathbf{8 . 8 2 0}}$

Total par semaine $\quad: 49,980$ 
Flux de données

\section{Transactions, D2/6-2.2}

REF. SOURCE: D2/6

REF. DESTIN.:

\section{2}

DESCRIPTION:

DESCRIPTION:
Transactions feuilles detemps

Valider Transactions feuilles-

\section{DESCRIPTION DÉTAILLÉE}

Contient toutes les transactions de feuilles de temps non-validées. Certaines de ces transactions auront le code de validation signifiant que la transaction a été acceptée, d'autres contiendront le code mentionnant que la transaction a été refusée.

\section{STRUCTURE DE DONNÉES INCLUSES}

numéro de la transaction: 5 caractères numériques

numéro de l'employé ou de l'associé: 3 caractères numériques

date de la transaction: 8 caractères alphanumériques

numéro du client: 8 caractères numériques

nom du client: 60 caractères alphanumériques

numéro de l'associé ${ }^{(1)}: 3$ caractères numériques

code de l'activité: 3 caractères numériques

temps: 4 caractères numériques (2 décimales)

description de l'activité: 40 caractères alphanumériques

code de validation: 1 caractère numérique 1 = transaction acceptée, $0=$ transaction refusée code d'imputation: 1 caractère numérique, $1=$ transaction traitée, $0=$ transaction non traitée

1- dans le cas de transactions facturables le numéro de l'associé comespond au numéro de l'associé auquel appartient le client, dans le cas de transactions non tacturables le numéro de l'associé correspond au numéro de l'associé duquel relève l'employé. 
VOLUME

Harel Drouin, et Associés $\quad: 35,280$

Harel Drouin Gestion Conseil : $\quad$ 5,880

Société d'informatique de gestion $: \underline{\mathbf{8 . 8 2 0}}$

Total par semaine $\quad: 49,980$ 
Flux de données

\section{Transactions erronées, 2.2-D2/1}

$\begin{array}{llll}\text { REF. SOURCE: } & 2.2 & \text { DESCRIPTION: } & \text { Valider feuilles de temps } \\ \text { REF. DESTIN.: } & \text { D2/1 } & \text { DESCRIPTION: } & \text { Rapport de validation }\end{array}$

DESCAIPTION DÉTAILLÉE:

Contient toutes les transactions pour lesquelles il n'y pas de numéro de client, dont le code d'activité $=000$ et celles pour lesquelles le nom du client est différent de celui qui est inscrit dans le fichier maître.

\section{STRUCTURE DE DONNÉES INCLUSES}

numéro de la transaction: 5 caractères numériques

numéro de l'employé ou de l'associé: 3 caractères numériques

date de la transaction: 8 caractères alphanumériques

numéro du client: 8 caractères numériques

nom du client: 60 caractères alphanumériques

numéro de l'associé ${ }^{(1)}$ : 3 caractères numériques

code de l'activité: 3 caractères numériques

temps: 4 caractères numériques ( 2 décimales) 
VOLUME

Harel Drouin, et Associés $\quad: 7^{(1)}$

Harel Drouin Gestion Conseil $\quad: 7$

Société d'informatique de gestion : $\underline{7}$

Total par semaine $\quad: 21$

1- 3 nouveaux clients +4 nouveaux employés 
Flux de données

\section{Transaction, D2/6-2.3}

$\begin{array}{llll}\text { REF. SOURCE: } & \text { D2/6 } & \text { DESCRIPTION: } & \text { Transactions feuilles detemps } \\ \text { REF. DESTIN.: } & 2.3 & \text { DESCRIPTION: } & \text { Corriger les transactions }\end{array}$

\section{DESCRIPTION DÉTAILLÉE}

Contient toute l'information concernant une transaction de feuille de temps. Cette fiche est lue lorsque nous procédons à la correction des fiches de transactions. Tous les champs de cette fiche peuvent être modifiés à l'exception du numéro de transaction.

\section{STRUCTURE DE DONNÉES INCLUSES}

numéro de la transaction: 5 caractères numériques

numéro de l'employé ou de l'associé: 3 caractères numériques

date de la transaction: 8 caractères alphanumériques

numéro du client: 8 caractères numériques

nom du client: 60 caractères alphanumériques

numéro de l'associé ${ }^{(1)}: 3$ caractères numériques

code de l'activité: 3 caractères numériques

temps: 4 caractères numériques ( 2 décimales)

description de l'activité: $\mathbf{4 0}$ caractères alphanumériques

code de validation: 1 caractère numérique

code d'imputation: 1 caractère numérique, 1 = transaction traitée, $0=$ transaction non traitée 


\section{VOLUME}

Harel Drouin, et Associés $\quad: 10$

Harel Drouin Gestion Conseil : 5

Société d'informatique de gestion : $\underline{5}$

Total par semaine $\quad: 20$ 
Flux de données

\section{Code, 2.3-D2/2}

$\begin{array}{llll}\text { REF. SOURCE: } & 2.3 & \text { DESCRIPTION: } & \text { Corriger les transactions } \\ \text { REF. DESTIN.: } & \text { D2/2 } & \text { DESCRIPTION: } & \text { Codes d'activités }\end{array}$

DESCRIPTION DÉTAILLÉE

Ce code d'activité est employé lorsqu'on modifie le code d'activité d'une transaction. Avec ce code, nous allons lire le nom de l'activité correspondant dans le fichier des codes d'activités. Le nom de l'activité est affiché sur l'écran afin que l'usager puisse le voir et qu'il vérifie l'exactitude de son entrée.

STRUCTURE DE DONNÉES INCLUSES

code de l'activité: 3 caractères numériques

\section{VOLUME}

Harel Drouin, et Associés $\quad: 10^{(1)}$

Harel Drouin Gestion Conseil : 5

Société d'informatique de gestion : $\underline{5}$

Total par semaine : : 20 
Flux de données

Nom, D2/2-2.3

REF. SOURCE: D2/2 DESCRIPTION: Codes d'activités

REF. DESTIN:: $\quad 2.3 \quad$ DESCRIPTION: $\quad$ Corriger les transactions

DESCRIPTION DÉTAILLÉE

Correspond au nom identifié à l'aide du code d'activité dans le fichier de codes d'activités $\mathrm{D} 2 / 2$.

STRUCTURE DE DONNÉES INCLUSES

nom: 60 caractères alphanumériques

VOLUME

Harel Drouin, et Associés $\quad: 35,280^{(1)}$

Harel Drouin Gestion Conseil : $\quad$ 5,880

Société d'informatique de gestion $: \underline{\mathbf{8 . 8 2 0}}$

Total par semaine $\quad: 49,980$

1- Etant donné que toutes les transactions peuvent être modifiés dans le processus 2.3 le volume maximal de ce flux peut atteidre 35,280 transactions. 
Flux de données

\section{Corrections, a.-2.3}

$\begin{array}{llll}\text { REF. SOURCE: } & \text { a. } & \text { DESCRIPTION: } & \text { Employés autorisés } \\ \text { REF. DESTIN.: } & 2.3 & \text { DESCRIPTION: } & \text { Corriger les transactions }\end{array}$

\section{DESCRIPTION}

Les corrections sont composées de nouveaux clients, de code d'activité signifiant que l'employé est non à l'emploi, de nom de clients pour lesquels le nom saisi ne correspondait pas à celui inscrit dans le fichier maître client ou de modification de n'importe quel champ d'une transaction.

\section{STRUCTURE DE DONNÉES INCLUSES}

1- nom du client: 60 caractères alphanumériques

2- un des champs suivants:

date de la transaction: 8 caractères alphanumériques numéro du client: 8 caractères numériques nom du client: 60 caractères alphanumériques code de l'activité: 3 caractères numériques temps: 4 caractères numériques ( 2 décimales) description de l'activité: 40 caractères alphanumériques code de validation: 1 caractère numérique, égal à 0 signifiant que la transaction est refusée. Elle doit repasser par le processus de validation. 
VOLUME

1- nom de nouveaux clients:

Harel Drouin, et Associés : 3

Harel Drouin Gestion Conseil : : 3

Société d'informatique de gestion $: \underline{3}$

Total par semaine $\quad: 9$

2- modifications de champs de transactions:

$\begin{array}{ll}\text { Harel Drouin, et Associés } & : 10^{(1)} \\ \text { Harel Drouin Gestion Conseil } & : 5 \\ \text { Société d'informatique de gestion } & : \underline{5} \\ \text { Total par semaine } & : 20\end{array}$


Flux de données

\section{Information client, 2.3-2.4}

$\begin{array}{llll}\text { REF. SOURCE: } & 2.3 & \text { DESCRIPTION: } & \text { Corriger les transactions } \\ \text { REF. DESTIN.: } & 2.4 & \text { DESCRIPTION: } & \text { Créer un nouveau client }\end{array}$

DESCRIPTION DÉTAILLÉE

Le numéro de l'associé, le numéro et le nom du client que nous désirons ajouter dans le fichier maître client.

\section{STRUCTURE DE DONNÉES INCLUSES}

numéro de l'associé: 3 caractères numériques

numéro du client: 8 caractères numériques

nom du client: 60 caractères alphanumériques

VOLUME

Harel Drouin, et Associés : 3

Harel Drouin Gestion Conseil : : 3

Société d'informatique de gestion : $\underline{3}$

Total par semaine $\quad: 9$ 
Flux de données

\section{Information client, 2.4-D2}

$\begin{array}{llll}\text { REF. SOURCE.: } & 2.4 & \text { DESCRIPTION: } & \text { Créer un nouveau client } \\ \text { REF. DESTIN.: } & \text { D2 } & \text { DESCRIPTION: } & \text { Fichier maître clients }\end{array}$

\section{DESCRIPTION DÉTAILLÉE}

Le numéro de l'associé, le numéro et le nom du client que nous désirons ajouter dans le fichier maître client.

STRUCTURE DE DONNÉES INCLUSES

numéro de l'associé: 3 caractères numériques

numéro du client: 8 caractères numériques

nom du client: 60 caractères alphanumériques

VOLUME

Harel Drouin, et Associés : 3

Harel Drouin Gestion Conseil : : 3

Société d'informatique de gestion $: \underline{3}$

Total par semaine $\quad: 9$ 
Flux de données

Message, 2.4-a.

$\begin{array}{llll}\text { REF. SOURCE: } & 2.4 & \text { DESCRIPTION: } & \text { Créer un nouveau client } \\ \text { REF. DESTIN.: } & \text { a. } & \text { DESCRIPTION: } & \text { Employés autorisés }\end{array}$

DESCRIPTION DÉTAILLÉE

Message indiquant qu'il existe dans le fichier maître client, un client ayant le même nom.

STRUCTURE DE DONNÉES INCLUSES

message: chaîne de caractères alphanumériques d'environ 60 caractères

$\begin{array}{lc}\text { VOLUME } & \\ \text { Harel Drouin, et Associés } & : 3 \\ \text { Harel Drouin Gestion Conseil } & : 3 \\ \text { Société d'informatique de gestion } & : \underline{3} \\ \text { Total par semaine } & : 9\end{array}$ 
Flux de données

\section{Numéro de l'associé, a-2.5}

$\begin{array}{llll}\text { REF. SOURCE: } & a & \text { DESCRIPTION: } & \text { Employés } \\ \text { REF. DESTIN.: } & 2.5 & \text { DESCRIPTION: } & \text { Imputer le temps }\end{array}$

DESCRIPTION DÉTAILLÉE

Ce flux contient le numéro de lassocié pour lequel nous désirons effectuer un mise à jour de ses travaux en cours.

STRUCTURE DE DONNÉES INCLUSES

numéro de l'associé: 3 caractères numériques

VOLUME

Harel Drouin, et Associés $\quad: 676^{(1)}$

Harel Drouin Gestion Conseil : : $208^{(2)}$

Société d'informatique de gestion $: 104^{(3)}$

Total par semaine $\quad$ : 998

1- 52 semaines * 13 associés

2- 52 semaines * 4 associés

3- 52 semaines * 2 associés 
Flux de données

\title{
Date et numéro de l'associé, 2.5-D2/7
}

\author{
REF. SOURCE: $2.5 \quad$ DESCRIPTION: Imputer le temps \\ REF. DESTIN.: D2/7 DESCAIPTION: Dernière date d'imputation
}

DESCRIPTION DÉTAILLÉE

Ce flux contient le nom d'un associé et la dernière date pour laquelle nous avons effectué une mise à jour de ses travaux en cours.

STRUCTURE DE DONNES INCLUSES

date: 8 caractères alphanumériques

numéro de l'associé: 3 caractères numériques

VOLUME

Harel Drouin, et Associés $\quad: 676^{\text {(1) }}$

Harel Drouin Gestion Conseil : $208^{(2)}$

Société d'informatique de gestion $: 104^{(3)}$

Total par semaine $\quad$ : 998

1- 52 semaines * 13 associés

2- 52 semaines * 4 associés

3- 52 semaines * 2 associés 
Flux de données

\section{Transactions, 2.7-D10}

$\begin{array}{llll}\text { REF. SOURCE: } & 2.7 & \text { DESCRIPTION: } & \text { Contrôler feuilles de temps } \\ \text { REF. DESTIN.: } & \text { D10 } & \text { DESCRIPTION: } & \text { Cumulatif des transactions }\end{array}$

\section{DESCRIPTION DÉTAILLÉE}

Toutes les transactions non facturables qui sont enregistrées dans le fichier transactions qui ont une des dates des journées comprises dans la semaine de feuille de temps.

\section{STRUCTURE DE DONNÉES INCLUSES}

numéro de la transaction: 5 caractères numériques

numéro de l'employé ou de l'associé: 3 caractères numériques

date de la transaction: 8 caractères alphanumériques

numéro du client: 8 caractères numériques

numéro de l'associé ${ }^{(1)}: 3$ caractères numériques

code de l'activité: 3 caractères numériques

temps: 4 caractères numériques ( 2 décimales)

description de l'activité: 40 caractères alphanumériques 
VOLUME

Harel Drouin, et Associés $\quad: 35,280$

Harel Drouin Gestion Conseil $\quad$ : 5,880

Société d'informatique de gestion $: \underline{8,820}$

Total par semaine $\quad: 49,980$ 
Flux de données

\section{Transactions facturables, 2.5-D7}

$\begin{array}{llll}\text { REF. SOURCE: } & 2.5 & \text { DESCRIPTION: } & \text { Imputer le temps } \\ \text { REF. DESTIN.: } & \text { D7 } & \text { DESCRIPTION: } & \text { Auxiliaire des travaux en cours }\end{array}$

\section{DESCRIPTION DÉTAILLÉE}

Toutes les transactions enregistrées dans le fichier transactions contenant le numéro de l'associé pour lequel une mise à jour a été demandée et dont le code d'activité signifie qu'elles représentent des activités facturables.

\section{STRUCTURE DE DONNÉES INCLUSES}

numéro du client: 5 caractères numériques

code de transaction ${ }^{(1)}: 1$ caractère numérique

numéro de l'écriture: 4 caractères numériques

numéro de la transaction: 5 caractères numériques

numéro de l'employé ou de l'associé: 3 caractères numériques

date de la transaction: $\mathbf{8}$ caractères alphanumériques

numéro du client: 8 caractères numériques

numéro de l'associé ${ }^{(2)}$ : 3 caractères numériques

$\begin{array}{ll}\text { 1- code de transaction: } & \text { (1) transaction au joumal général } \\ \text { (2) transaction au joumal des revenus } \\ \text { (3) transaction au joumal de la facturation } \\ \text { (4) transaction au caisse recettes } \\ \text { (5) transaction au caise déboursés } \\ \text { (6) transaction au joumal des acahts }\end{array}$

2- dans le cas de transactions facturables le numéro de l'associé correspond au numéro de l'associé auquel appartient le client, dans le cas de transactions non facturables le numéro de l'associé correspond au numéro de l'associé duquel relève l'employé. 
code de l'activité: 3 caractères numériques temps: 4 caractères numériques (2 décimales)

description de l'activité: $\mathbf{4 0}$ caractères alphanumériques

taux horaire: 3 caractères numériques

total: 5 caractères numériques

VOLUME

Harel Drouin, et Associés $\quad: 26,460^{(1)}$

Harel Drouin Gestion Conseil $\quad$ : $\quad \mathbf{4 , 4 1 0}$

Société d'informatique de gestion $: \underline{6.615}$

Total par semaine $\quad: 37,485$ 
Flux de données

\section{Numéro de l'associe, D1-2.5}

$\begin{array}{llll}\text { REF. SOURCE: } & \text { D1 } & \text { DESCRIPTION: } & \begin{array}{l}\text { Fichier maître employés } \\ \text { associés }\end{array} \\ \text { REF. DESTIN.: } & 2.5 & \text { DESCRIPTION: } & \text { Imputer le temps }\end{array}$

\section{DESCRIPTION DÉTAILLÉE}

Ce flux est utilisé pour valider le numéro de l'associé fournit par l'employé autorisé (a.). Une fois que cet employé a fournit au système le numéro de l'associé pour lequel une mise à jour des travaux en cours est demandée, le système vérifie si l'associé existe vraiment. Si l'associé n'est pas inscrit dans le fichier maître associés employés, un message d'erreur sera affiché.

STRUCTURE DE DONNÉES INCLUSES

numéro de l'associé: 3 caractères numériques

\section{VOLUME}

Harel Drouin, et Associés $\quad: 676^{(1)}$

Harel Drouin Gestion Conseil $\quad$ : $208^{(2)}$

Société d'informatique de gestion : $104^{(3)}$

Total par semaine $\quad: 156$

1- 52 semaines * 13 associés

2- 52 semaines * 4 associés

3- 52 semaines * 2 associés 
Flux de données

\section{Transactions, D10-2.6}
REF. SOURCE: D10
DESCRIPTION:
Cumulatif des transactions
REF. DESTIN.:
2.6
DESCRIPTION:
Produire rapport statistique

\section{DESCRIPTION DÉTAILLÉE}

Toutes les transactions qui sont enregistrées dans le fichier cumulatif des transactions $\mathrm{D} 2 / 4$.

\section{STRUCTURE DE DONNÉES INCLUSES}

numéro de la transaction: 5 caractères numériques

numéro de l'employé ou de l'associé: 3 caractères numériques

date de la transaction: 8 caractères alphanumériques

numéro du client: 8 caractères numériques

numéro de l'associé ${ }^{(1)}: 3$ caractères numériques

code de l'activité: 3 caractères numériques

temps: 4 caractères numériques ( 2 décimales)

description de l'activité: $\mathbf{4 0}$ caractères alphanumériques 
VOLUME

Harel Drouin, et Associés $\quad: 1,834,560^{(1)}$

Harel Drouin Gestion Conseil : $\quad 305,760$

Société d'informatique de gestion : 458,640

Total par semaine $\quad: 2,617,305$ 
Flux de données

\title{
Informations pour rapports, C-2.6
}

\author{
REF. SOURCE: C DESCRIPTION: Associés \\ $\begin{array}{llll}\text { REF. DESTIN.: } & 2.6 & \text { DESCRIPTION: } & \text { Produire rapport statistique }\end{array}$
}

\section{DESCRIPTION DÉTAILLÉE}

Le numéro de l'associé et les dates pour lesquelles on désire produire le rapport.

STRUCTURE DE DONNÉES INCLUSES

numéro de l'associé: 3 caractères numériques

date de début du rapport: 8 caractères alphanumériques

date de la fin du rapport: 8 caractères alphanumériques

VOLUME

Harel Drouin, et Associés $\quad: 12^{(1)}$

Harel Drouin Gestion Conseil $\quad: 4$

Société d'informatique de gestion : $\underline{4}$

Total par semaine $\quad: 20$ 
Flux de données

\section{Rapport statistique, 2.6-c}

$\begin{array}{llll}\text { REF. SOURCE: } & 2.6 & \text { DESCRIPTION: } & \text { Produire rapport statistiques } \\ \text { REF. DESTIN:: } & \text { C } & \text { DESCRIPTION: } & \text { Associés }\end{array}$

\section{DESCRIPTION DÉTAILLÉE}

Rapport fournissant de l'information sur le temps facturable et non facturable pour chaque employé, pour chaque équipe ou pour l'entreprise.

STRUCTURE DE DONNÉES INCLUSES

voir section 2.6 pour de plus amples détails

VOLUME

Harel Drouin, et Associés $\quad: 12^{(1)}$

Harel Drouin Gestion Conseil : : 4

Société d'informatique de gestion : 4

Total par semaine $\quad: 20$ 
Flux de données

\section{Coordonnées, D2/3-2.7}

$\begin{array}{llll}\text { REF. SOURCE: } & D 2 / 3 & \text { DESCRIPTION: } & \text { Coordonnées feuilles de temps } \\ \text { REF. DESTIN.: } & 2.7 & \text { DESCRIPTION: } & \text { Contrôler feuilles de temps }\end{array}$

\section{DESCRIPTION DÉTAILLÉE}

Ce flux contient les dates de feuilles de temps et des journées incluses dans chacune des feuilles de temps. Pour chaque personne identifiée dans le fichier maître associés employés, il doit avoir, dans le fichier transactions, une transaction ayant le numéro de l'employé ou de l'associé et une date correspondant à une des dates lues dans le fichier des coordonnées de feuilles de temps.

\section{STRUCTURE DE DONNÉES INCLUSES}

date de la feuille de temps: 8 caractères alphanumériques

1 ère journée: 8 caractères alphanumériques

nombre d'heures régulières pour la première journée: 1 caractère numérique 2 ème journée: 8 caractères alphanumériques nombre d'heures régulières pour la deuxième journée: 1 caractère numérique 3 ème journée: 8 caractères alphanumériques nombre d'heures régulières pour la troisième journée: 1 caractère numérique 4 ème journée: 8 caractères alphanumériques nombre d'heures régulières pour la quatrième journée: 1 caractère numérique 5 ème journée: 8 caractères alphanumériques nombre d'heures régulières pour la cinquième journée: 1 caractère numérique 6 ème journée: 8 caractères alphanumériques 
nombre d'heures régulières pour la sixième journée: 1 caractère numérique

7 ème journée: 8 caractères alphanumériques

nombre d'heures régulières pour la septième journée: 1 caractère numérique

VOLUME

Harel Drouin, et Associés $\quad: 52^{(1)}$

Harel Drouin Gestion Conseil : 52

Société d'informatique de gestion : 52

Total par semaine $\quad: 156$

1- 52 semaines par années 
Flux de données

Feuilles complétées, 2.7-D2/3

$\begin{array}{llll}\text { REF. SOURCE: } & 2.7 & \text { DESCRIPTION: } & \text { Contrôler feuilles de temps } \\ \text { REF. DESTIN.: } & \text { D2/3 } & \text { DESCRIPTION: } & \text { Coordonnées feuilles de temps }\end{array}$

\section{DESCRIPTION}

Ce flux contient un code qui est inscrit dans la fiche de coordonnées d'une feuille de temps afin d'informer le système que toutes les feuilles de temps ont été complétées pour la semaine.

STRUCTURE DE DONNÉES INCLUSES

code signifiant que la feuille est complète: 1 caractère numérique.

VOLUME

Harel Drouin, et Associés $\quad: 52^{(1)}$

Harel Drouin Gestion Conseil : 52

Société d'informatique de gestion : 52

Total par semaine $\quad: 156$ 
Flux de données

\section{Identification, 2.7-D2/5}
REF. SOURCE:
2.7
DESCRIPTION:
Contrôler feuille de temps
REF. DESTIN.:
$\mathrm{D} 2 / 5$
DESCRIPTION:
Feuilles de temps manquantes

\section{DESCRIPTION DÉTAILLÉE}

En fait ce flux représente l'information relative aux personnes qui n'ont pas produit leur feuille de temps. II contient, pour chaque individu n'ayant pas produit sa feuille de temps, le numéro de l'associé, le numéro d'identification et le nom de toutes les personnes qui n'ont pas produit leur feuille de temps.

\section{STRUCTURE DE DONNÉES INCLUSES}

date de la feuille de temps: 8 caractères alphanumériques

pour chacune des personnes n'ayant pas produit sa feuille de temps:

numéro de l'associé: 3 caractères numériques

numéro de la personne: 3 caractères numériques

nom de la personne: 20 caractères alphanumériques

prénom de la personne: 20 caractères alphanumériques 
VOLUME

Harel Drouin, et Associés $\quad: 18^{(1)}$

Harel Drouin Gestion Conseil : : 3

Société d'informatique de gestion : $\underline{5}$

Total par semaine $\quad: 26$

1- environ $10 \%$ personnes ne remettent pas leur feuilles de temps dans les délais demandés 
Flux de données

Feuilles de temps manquantes, 2.7-A

$\begin{array}{llll}\text { REF. SOURCE: } & 2.7 & \text { DESCRIPTION: } & \text { Contrôler feuille de temps } \\ \text { REF. DESTIN.: } & \text { A } & \text { DESCRIPTION: } & \text { Employés }\end{array}$

DESCRIPTION DÉTAILLÉE

En fait ce flux représente un rapport contenant la date de la feuille de temps pour laquelle le rapport est produit et le numéro d'équipe, le numéro d'identification et le nom de toutes les personnes qui n'ont pas produit leur feuille de temps.

STRUCTURE DE DONNÉES INCLUSES

date de la feuille de temps: 8 caractères alphanumériques

pour chacune des personnes n'ayant pas produit sa feuille de temps:

numéro de l'associé: 3 caractères numériques

numéro de la personne: 3 caractères numériques

nom de la personne: 20 caractères alphanumériques

prénom de la personne: 20 caractères alphanumériques 
VOLUME

Harel Drouin, et Associés $\quad: 18^{(1)}$

Harel Drouin Gestion Conseil : : 3

Société d'informatique de gestion : $\underline{5}$

Total par semaine $\quad: 26$

1- environ $10 \%$ personnes ne remettent pas leur feuilles de temps dans les délais demandés 
Flux de données

\title{
Date de la feuille de temps, A-2.7
}

\author{
REF. SOURCE: A DESCRIPTION: Employés \\ REF. DESTIN.: $\quad 2.7 \quad$ DESCRIPTION: $\quad$ Contrôler feuilles de temps
}

\section{DESCRIPTION DÉTAILLÉE}

Ce flux contient la date de la feuille de temps pour laquelle nous désirons effectuer un contrôle des feuilles de temps. Étant donné que le contrôle des feuilles de temps est composé de 3 processus (déterminer les feuilles de temps manquantes et vérifier les feuilles de temps manquantes, calculer le temps supplémentaire) dont deux sont transparents à l'utilisateur, ce flux sert à sélectionner les enregistrements qui seront imprimés ou affichés par le processus 2.7.2 vérifier les feuilles de temps manquantes.

\section{DESCRIPTION DÉTAILLÉE}

date: 8 caractères numériques

\section{VOLUME}

Harel Drouin, et Associés : $52^{(1)}$

Harel Drouin Gestion Conseil $\quad$ : 52

Société d'informatique de gestion : 52

Total par semaine $\quad: 156$ 
Flux de données

\section{Numéro de l'associé, a.-2.7}

$\begin{array}{llll}\text { REF. SOURCE: } & \text { a. } & \text { DESCRIPTION: } & \text { Employés autorisés } \\ \text { REF. DESTIN.: } & 2.7 & \text { DESCRIPTION: } & \text { Contrôler feuilles de temps }\end{array}$

\section{DESCRIPTION DÉTAILLÉE}

Ce flux est employé conjointement avec la date de la feuille de temps pour sélectionner, parmi les personnes qui n'ont pas remis leurs feuilles de temps dans les délais prescrits, les noms des personnes qui seront affichés à l'écran ou imprimés.

STRUCTURE DE DONNÉES INCLUSES

numéro de l'associé: 3 caractères numériques

VOLUME

Harel Drouin, et Associés $\quad: 676^{(1)}$

Harel Drouin Gestion Conseil : $208^{(2)}$

Société d'informatique de gestion $: \underline{104}^{(3)}$

Total par semaine $\quad$ : 988

\footnotetext{
1- 52 semaines * 13 associés

2- 52 semaines * 4 associés

3- 52 semaines * 2 associés
} 
Flux de données

Identification, D1-2.7

REF. SOURCE: D1 DESCRIPTION:

Fichier maître employés associés

REF. DESTIN.: $\quad 2.7 \quad$ DESCRIPTION:

Contrôler feuilles de temps manquante

\section{DESCRIPTION DÉTAILLÉE}

En fait ce flux représente l'information relative aux personnes qui sont enregistrées dans le fichier maître employés associés. II contient, pour chaque individu n'ayant pas produit sa feuille de temps, le numéro de l'associé, le numéro d'identification et le nom.

A l'aide du numéro de l'associé, l'employé autorisé demande d'afficher ou d'imprimer l'identification de toutes les personnes n'ayant pas produit leur feuille de temps, et ce, pour le numéro d'associé fournit.

VOLUME

Harel Drouin, et Associés $\quad: 18^{(1)}$

Harel Drouin Gestion Conseil : : 3

Société d'informatique de gestion : $\underline{5}$

Total par semaine $\quad: 26$ 
Flux de données

\title{
Rapport de validation, D2/1-a.
}

\author{
REF. SOURCE: D2/1 DESCRIPTION: Rapport de validation \\ REF. DESTIN.: a. DESCRIPTION: Employés autorisés
}

\section{DESCRIPTION DÉTAILLÉE}

Ce flux contient toutes les transactions dont le code d'activité $=000$ (non à l'emploi) et celles qui ont été refusées pour un des raisons suivantes:

pas de numéro de client

le nom du client inscrit dans le transaction n'est pas le même que celui inscrit dans le fichier maître client.

\section{STRUCTURE DE DONNÉES INCLUSES}

numéro de la transaction: 5 caractères numériques

numéro de l'employé ou de l'associé: 3 caractères numériques

date de la transaction: 8 caractères alphanumériques

numéro du client: 8 caractères numériques

nom du client: 60 caractères alphanumériques

temps: 4 caractères numériques ( 2 décimales) 
VOLUME

Harel Drouin, et Associés $\quad: 7^{(1)}$

Harel Drouin Gestion Conseil : : 7

Société d'informatique de gestion : 7

Total par semaine $\quad: 21$

1- 3 nouveaux clients +4 nouveaux employés 
Flux de données

\section{Rapport de validation, D2/1-2.5}

$\begin{array}{llll}\text { REF. SOURCE: } & \text { D2/1 } & \text { DESCRIPTION: } & \text { Rapport de validation } \\ \text { REF. DESTIN.: } & 2.5 & \text { DESCRIPTION: } & \text { Imputer le temps }\end{array}$

\section{DESCRIPTION DÉTAILLÉE}

Ce flux contient toutes les transactions dont le code d'activité $=000$ (non à l'emploi) et celles qui ont été refusées pour un des raisons suivantes:

pas de numéro de client

le nom du client inscrit dans le transaction n'est pas le même que celui inscrit dans le fichier maître client.

Lors de l'imputation des feuilles de temps (processus 2.5), le système regarde dans le fichier D2/1 s'il y a des transactions ayant le numéro de l'associé pour lequel l'imputation des transactions est demandée. Dans l'affirmative, le système informera l'utilisateur de ce fait et lui demandera s'il désire quand même procéder à l'imputation des transactions.

\section{STRUCTURE DE DONNÉES INCLUSES}

numéro de la transaction: 5 caractères numériques

numéro de l'employé ou de l'associé: 3 caractères numériques

date de la transaction: 8 caractères alphanumériques

numéro du client: 8 caractères numériques

nom du client: 60 caractères alphanumériques

temps: 4 caractères numériques ( 2 décimales) 
VOLUME

Harel Drouin, et Associés $\quad: 7^{(1)}$

Harel Drouin Gestion Conseil : : 7

Société d'informatique de gestion : $\underline{7}$

Total par semaine $\quad: 21$

1- 3 nouveaux clients +4 nouveaux employés 
Flux de données

\section{Identification, D2/5-2.5}

REF. SOURCE: D2/5 DESCRIPTION:

REF. DESTIN.: $\quad 2.5$
2.5 DESCRIPTION:
Feuilles de temps manquantes

Imputer le temps

\section{DESCRIPTION DÉTAILÉE}

En fait ce flux représente l'information relative aux personnes qui n'ont pas produit leur feuille de temps. II contient, pour chaque individu n'ayant pas produit sa feuille de temps, le numéro de l'associé, le numéro d'identification et le nom de toutes les personnes qui n'ont pas produit leur feuille de temps.

Lors de l'imputation des feuilles de temps (processus 2.5), le système regarde dans le fichier D2/5 s'il y a des personnes ayant le numéro de l'associé pour lequel l'imputation des transactions est demandée. Dans l'affirmative, le système informera l'utilisateur de ce fait et lui demandera s'il désire quand même procéder à l'imputation des transactions.

\section{STRUCTURE DE DONNÉES INCLUSES}

date de la feuille de temps: 8 caractères alphanumériques

pour chacune des personnes n'ayant pas produit sa feuille de temps:

numéro de l'associé: 3 caractères numériques

numéro de la personne: 3 caractères numériques

nom de la personne: 20 caractères alphanumériques

prénom de la personne: 20 caractères alphanumériques 


\section{VOLUME}

Harel Drouin, et Associés $\quad: 18^{(1)}$

Harel Drouin Gestion Conseil : : 3

Société d'informatique de gestion : $\underline{5}$

Total par semaine $\quad: 26$

1- environ $10 \%$ personnes ne remettent pas leur feuilles de temps dans les délais demandés 
Flux de données

\section{Temps supplémentaire, 2.7-D2/4}

$\begin{array}{llll}\text { REF. SOURCE: } & 2.7 & \text { DESCRIPTION: } & \text { Contrôler feuilles de temps } \\ \text { REF. DESTIN.: } & \text { D2/4 } & \text { DESCRIPTION: } & \text { Cumulatif des transactions }\end{array}$

\section{DESCRIPTION DÉTAILLÉE}

Ce flux contient des transactions non facturables. Le code d'activité signifie que la transaction représentent du temps supplémentaire et le temps de l'activité à été calculé lors de l'exécution du processus 2.7. Ce flux, est généré par le processus 2.7 seulement lorsque le temps supplémentaire est différent de 0 (plus petit ou plus grand).

\section{STRUCTURE DE DONNÉES INCLUSES}

numéro de la transaction: 5 caractères numériques

numéro de l'employé ou de l'associé: 3 caractères numériques

numéro de l'associé (équipe): 3 caractères numériques

date de la transaction: 8 caractères alphanumériques

code de l'activité: 3 caractères numériques

temps: 4 caractères numériques ( 2 décimales)

\section{VOLUME}

Harel Drouin, et Associés $\quad: 27,000^{(1)}$

Harel Drouin Gestion Conseil : : $\quad$ 4,500

Société d'informatique de gestion : $\underline{6,750}$

Total par semaine

: 38,250

1- 150 journées * 180 employés 
Flux de données

\section{Transactions, D2/6-2.7}

$\begin{array}{llll}\text { REF. SOURCE: } & \text { D2/6 } & \text { DESCRIPTION: } & \text { Transactions feuilles detemps } \\ \text { REF. DESTIN.: } & 2.7 & \text { DESCRIPTION: } & \text { Contrôler feuilles de temps }\end{array}$

\section{DESCRIPTION DÉTAILLÉE}

Ce flux contient toutes les transactions qui sont utilisées par le processus 2.7 Contrôler feuilles de temps. Ce processus lit tous les enregistrements du fichier maître employésassociés. Pour chaque enregistrement lu, le processus cherche, dans le fichier des transactions, une transaction ayant le numéro de l'employé ou de l'associé qui a été lu dans le fichier maître. Si aucune transaction n'est trouvé, le système considère que la personne n'a pas remis sa feuille de temps. 
STRUCTURE DE DONNÉES INCLUSES

numéro de la transaction: 5 caractères numériques

numéro de l'employé ou de l'associé: 3 caractères numériques

date de la transaction: 8 caractères alphanumériques

numéro du client: 8 caractères numériques

nom du client: 60 caractères alphanumériques

numéro de l'associé ${ }^{(1)}: 3$ caractères numériques

code de l'activité: 3 caractères numériques

temps: 4 caractères numériques ( 2 décimales)

description de l'activité: $\mathbf{4 0}$ caractères alphanumériques

code de validation: 1 caractère numérique

code d'imputation: 1 caractère numérique, $1=$ transaction traitée, $0=$ transaction non traitée

\section{VOLUME}

Harel Drouin, et Associés $\quad: 35,280$

Harel Drouin Gestion Conseil $\quad$ : 5,880

Société d'informatique de gestion $: \underline{8.820}$

Total par semaine $\quad: 49,980$ 
Flux de données

Nom, D1-2.6

REF. SOURCE:

D1

DESCRIPTION:

Fichier maître employésassociés

REF. DESTIN.:

DESCRIPTION:

Produire rapport statistique

\section{DESCRIPTION DÉTAILLÉE}

C'est le nom correspondant au numéro de l'employé ou de l'associé qui est enregistré dans le champ nom d'une transaction.

STRUCTURE DE DONNÉES INCLUSES

nom: 20 caractères alphanumériques

prénom: 20 caractères alphanumériques

\section{VOLUME}

Harel Drouin, et Associés $\quad: 180$

Harel Drouin Gestion Conseil : 30

Société d'informatique de gestion : $\underline{45}$

Total par semaine $\quad: 255$ 
Flux de données

\section{Transactions, D2/6-2.5}

$\begin{array}{llll}\text { REF. SOURCE: } & \text { D2/6 } & \text { DESCRIPTION: } & \text { Transactions feuilles detemps } \\ \text { REF. DESTIN.: } & 2.5 & \text { DESCRIPTION: } & \text { Imputer le temps }\end{array}$

\section{DESCRIPTION DÉTAILLÉE}

Ce flux contient toutes les transactions lues dans le fichier transactions. Si la transaction n'est pas validée, elle n'est pas utilisée pour mettre à jour l'auxiliaire des travaux en cours et n'est pas inscrite dans le fichier D2/4 Cumulatif des transactions. II faut prendre note que seulement les transactions dont le code de validation est égale à 0 peuvent être imputées.

STRUCTURE DE DONNÉES INCLUSES

numéro de la transaction: 5 caractères numériques

numéro de l'employé ou de l'associé: 3 caractères numériques

date de la transaction: 8 caractères alphanumériques

numéro du client: 8 caractères numériques

nom du client: 60 caractères alphanumériques

code de l'activité: 3 caractères numériques

temps: 4 caractères numériques (2 décimales)

description de l'activité: $\mathbf{4 0}$ caractères alphanumériques

code de validation: 1 caractère numérique

code d'imputation: 1 caractère numérique, $1=$ transaction traitée, $0=$ transaction non traitée 
VOLUME

Harel Drouin, et Associés $\quad: 35,280$

Harel Drouin Gestion Conseil $\quad$ : 5,880

Société d'informatique de gestion : $\mathbf{8 , 8 2 0}$

Total par semaine $\quad: 49,980$ 
Flux de données

\section{Transactions, 2.5-D2/6}

$\begin{array}{llll}\text { REF. SOURCE: } & 2.5 & \text { DESCRIPTION: } & \text { Imputer le temps } \\ \text { REF. DESTIN: } & \text { D2/6 } & \text { DESCRIPTION: } & \text { Transactions feuilles detemps }\end{array}$

DESCRIPTION DÉTAILLÉE

Ce flux contient toutes les transactions lues dans le fichier transactions. Si la transaction n'est pas validée, elle n'est pas utilisée pour mettre à jour l'auxiliaire des travaux en cours et n'est pas inscrite dans le fichier D2/4 Cumulatif des transactions. Lorsque la transaction est traitée, son code d'imputation est égal à 1 .

STRUCTURE DE DONNÉES INCLUSES

numéro de la transaction: 5 caractères numériques

numéro de l'employé ou de l'associé: 3 caractères numériques

date de la transaction: 8 caractères alphanumériques

numéro du client: 8 caractères numériques

nom du client: 60 caractères alphanumériques

code de l'activité: 3 caractères numériques

temps: 4 caractères numériques ( 2 décimales)

description de l'activité: 40 caractères alphanumériques

code de validation: 1 caractère numérique

code d'imputation: 1 caractère numérique, $1=$ transaction traitée, $0=$ transaction non traitée 


\section{VOLUME}

Harel Drouin, et Associés $\quad: 35,280$

Harel Drouin Gestion Conseil $\quad$ : 5,880

Société d'informatique de gestion $: \underline{8.820}$

Total par semaine $\quad: 49,980$ 
Flux de données

\section{Information client, a.-2.4}

REF. SOURCE:

a. DESCRIPTION:

Employés autorisés

REF. DESTIN.:

2.4 DESCRIPTION:

Créer un nouveau client

\section{DESCRIPTION DÉTAILLÉE}

Le numéro de l'associé, le numéro et le nom du client que nous désirons ajouter dans le fichier maître client.

STRUCTURE DE DONNÉES INCLUSES

numéro de l'associé: 3 caractères numériques

numéro du client: 8 caractères numériques

nom du client: 60 caractères alphanumériques

$\begin{array}{ll}\text { VOLUME } & \\ \text { Harel Drouin, et Associés } & : 3 \\ \text { Harel Drouin Gestion Conseil } & : 3 \\ \text { Société d'informatique de gestion } & : \underline{3} \\ \text { Total par semaine } & : 9\end{array}$


Flux de données

\title{
Transaction, 2.3-D2/6
}

\author{
$\begin{array}{llll}\text { REF. SOURCE: } & 2.3 & \text { DESCRIPTION: } & \text { Corriger les transactions }\end{array}$ \\ REF. DESTIN.: $\quad$ D2/6 \\ DESCRIPTION: \\ Transactions feuilles detemps
}

\section{DESCRIPTION DÉTAILLÉE}

Contient toute l'information concernant une transaction modifiée de feuille de temps. Cette fiche est lue lorsque nous procédons à la correction des fiches de transactions. Tous les champs de cette fiche peuvent avoir été modifiés à l'exception du numéro de transaction.

\section{STRUCTURE DE DONNÉES INCLUSES}

numéro de la transaction: 5 caractères numériques

numéro de l'employé ou de l'associé: 3 caractères numériques

date de la transaction: 8 caractères alphanumériques

numéro du client: 8 caractères numériques

nom du client: 60 caractères alphanumériques

numéro de l'associé ${ }^{(1)}: 3$ caractères numériques

code de l'activité: 3 caractères numériques

temps: 4 caractères numériques ( 2 décimales)

description de l'activité: 40 caractères alphanumériques

code de validation: 1 caractère numérique

code d'imputation: 1 caractère numérique, $1=$ transaction traitée, $0=$ transaction non traitée

1- dans le cas de transactions facturables le numéro de l'associé correspond au numéro de l'associé auquel appartient le client, dans le cas de transactions non facturables le numéro de l'associé correspond au numéro de l'associé duquel relève l'employé. 
VOLUME

Harel Drouin, et Associés $\quad: 10$

Harel Drouin Gestion Conseil : 5

Société d'informatique de gestion : $\underline{5}$

Total par semaine $\quad: 20$ 
Flux de données

\section{Taux horaire, D1-2.5}

$\begin{array}{llll}\text { REF. SOURCE: } & \text { D1 } & \text { DESCRIPTION: } & \begin{array}{l}\text { Fichier maître employés } \\ \text { associés }\end{array} \\ \text { REF. DESTIN.: } & 2.5 & \text { DESCRIPTION: } & \text { Imputer le temps }\end{array}$

\section{DESCRIPTION DÉTAILLÉE}

C'est le taux horaire de chaque individu. Ce taux est inscrit dans le fichier maître employés associés et, est lu pour inscrire les transactions dans l'auxiliaire des travaux en cours.

STRUCTURE DE DONNÉES INCLUSES

taux horaire: 3 caractères numériques

VOLUME

Harel Drouin, et Associés $\quad: 26,460^{(1)}$

Harel Drouin Gestion Conseil $\quad$ : 4,410

Société d'informatique de gestion : $\underline{6.615}$

Total par semaine $\quad: 37,485$ 
Flux de données

$$
\text { \#, D1-2.8 }
$$

REF. SOURCE: D1

DESCRIPTION:

Fichier maître employésassociés

REF. DESTIN.: $\quad 2.8$

DESCRIPTION: Saisir données feuilles de temps

\section{DESCRIPTION}

C'est le numéro de l'employé. II est utilisé à l'intérieur du processus 2.8 pour valider le numéro de l'employé ou associé saisi.

STRUCTURE DE DONNÉES INCLUSES

numéro: 3 caractères numériques

VOLUME

Harel Drouin, et Associés $\quad: 35,280$

Harel Drouin Gestion Conseil $\quad$ : 5,880

Société d'informatique de gestion $: \underline{\mathbf{8 . 8 2 0}}$

Total par semaine $\quad: 49,980$ 
Flux de données

\title{
Écritures de journal, 2.5-D8
}

\author{
REF. SOURCE: $2.5 \quad$ DESCRIPTION: Imputer le temps \\ REF. DESTIN.: D8 DESCRIPTION: Écritures de journal
}

\section{DESCRIPTION}

Ce flux contient l'information relative aux revenus. II contient une écritures de journal qui affectent les comptes de revenus et de travaux en cours.

\section{STRUCTURE DE DONNÉES INCLUSES}

numéro de l'écriture: 5 caractères numériques

code de transaction ${ }^{(1)}: 1$ caractère numérique

date de la transaction: 8 caractères numériques

numéro de compte: 4 caractères numériques

numéro du sous-compte: 2 caractères numériques

montant: 7 caractères numériques ( 2 décimales)

description: 60 caractères numériques 
VOLUME

Harel Drouin, et associés : entre 52 et 624

Harel Drouin Gestion Conseil : entre 52 et 208

Société d'informatique de gestion : entre 52 et 104

Total par semaine $\quad$ : entre 156 et 936 
Flux de données

\section{\# écritures, D9-2.5}

REF. SOURCE: D9 DESCRIPTION: Numéro d'écriture

REF. DESTIN.: $2.5 \quad$ DESCRIPTION: $\quad$ Imputer le temps

DESCRIPTION DÉTAILLÉE

C'est un numéro qui est généré par le système. Ce numéro permet de retracer la trace des enregistrements effectués dans les comptes du grand-livre.

STRUCTURE DE DONNÉES INCLUSES

numéro: 5 caractères numériques

VOLUME

Harel Drouin, et associés $\quad$ : entre 52 et 624

Harel Drouin Gestion Conseil : : entre 52 et 208

Société d'informatique de gestion : entre 52 et 104

Total par semaine : : entre 156 et 936 
Flux de données

\section{Transactions, D7-2.6}
REF. SOURCE:
D7
DESCRIPTION:
Auxiliaire des travaux en cours
REF. DESTIN.:
DESCRIPTION:
Produire rapport statistique

\section{DESCRIPTION DÉTAILLÉE}

Contient des transactions qui sont lues dans l'auxiliaire des travaux en cours.

\section{STRUCTURE DE DONNÉES INCLUSES}

numéro de la transaction: 5 caractères numériques

numéro de l'employé ou de l'associé: 3 caractères numériques

date de la transaction: 8 caractères alphanumériques

numéro du client: 8 caractères numériques

numéro de l'associé (1) $: 3$ caractères numériques

code de l'activité: 3 caractères numériques

temps: 4 caractères numériques ( 2 décimales)

description de l'activité: $\mathbf{4 0}$ caractères alphanumériques

taux horaire: 3 caractères numériques

total: 5 caractères numériques

1- dans le cas de transactions facturables le numéro de l'associé correspond au numéro de l'associé auquel appartient le client, dans le cas de transactions non facturables le numéro de l'associé correspond au numéro de l'associé duquel relève l'employé. 
VOLUME

Harel Drouin, et Associés

: $26,460 * 52$ semaines $^{(1)}$

Harel Drouin Gestion Conseil $\quad$ : $\quad 4,410 * 52$ semaines

Société d'informatique de gestion : $\quad 6,615$ * 52 semaines

Total par semaine

$: 1,949,220$

1- environ les 3/4 des transactions sont facturables 
Processus 2.1, Produire les feuilles de temps 
Diagramme de flux d'information 
2.1 Produire les feuille de temps

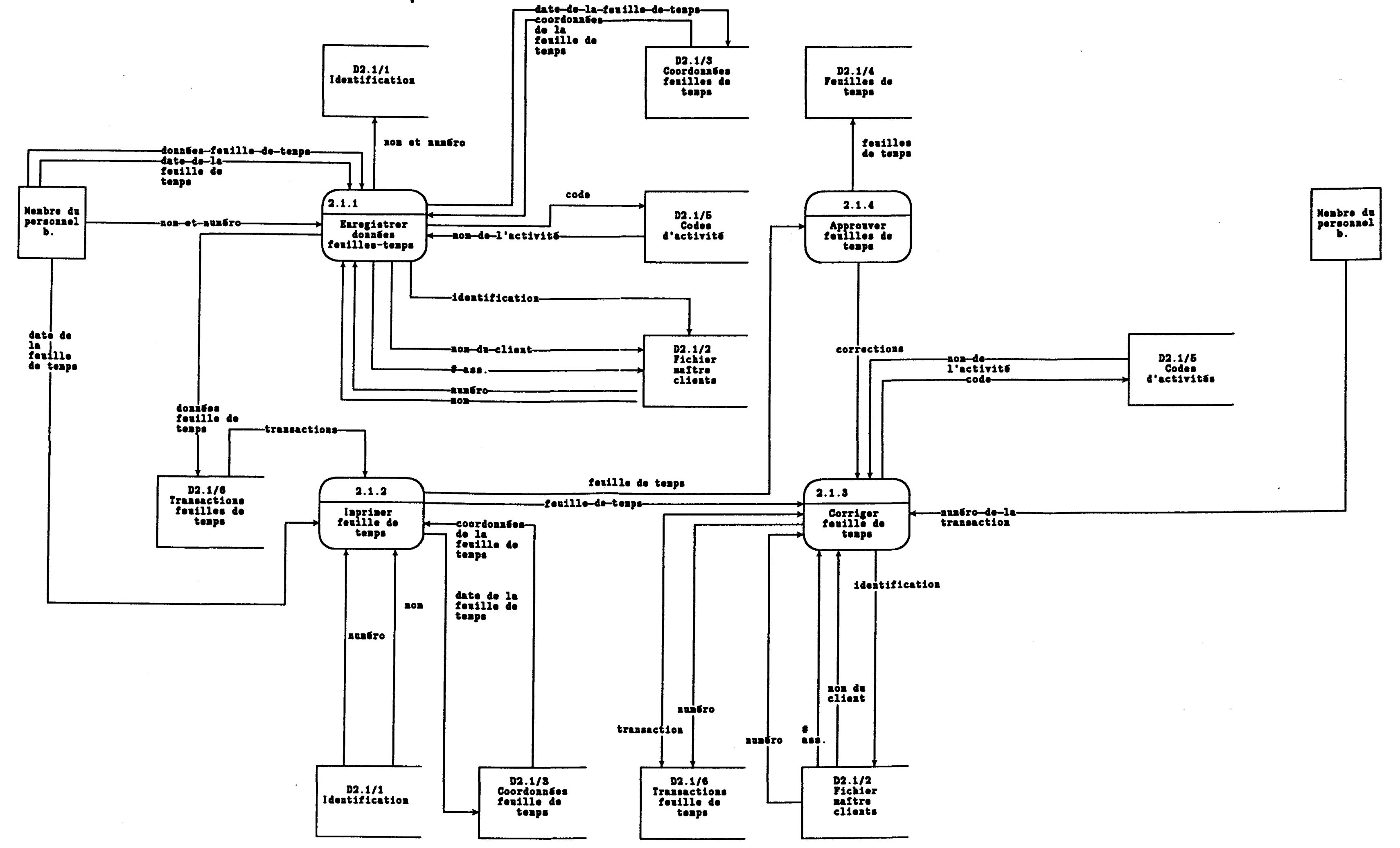




\section{Processus de traitement}


Processus de traitement

\section{Enregistrer données feuilles-temps, 2.1.1}

\section{DESCRIPTION}

Cette opération consiste à enregistrer toutes les informations nécessaires à la production d'une feuille de temps. Pour enregistrer cette information, la date de la feuille de temps fournie par l'usager doit être inscrite dans le fichier des coordonnées de feuilles de temps. Le code d'activité doit être enregistré dans le fichier des codes d'activités.

\section{ENTRÉES}

Numéro, D2.1/2-2.1.1

Données feuilles-temps, b-2.1.1

Date feuille de temps, b-2.1.1

Nom et numéro, b-2.1.1

Nom, D2.1/2-2.1.1

Coordonnées de la feuille de Temps,

D2.1/3-2.1.1

Nom de l'activité, D2.1/5-2.1.1
SORTIE

Données feuille de temps, 2.1.1-D2.1/6

Code, 2.1.1-D2.1/5

Date de la feuille de temps, 2.1.1-D2.$1 / 3$

Nom du client, 2.1.1-D2.1/2

Identification, 2.1.1-D2.1/2

Nom et numéro, 2.1.1-D2.1/1

\# ass.,2.1.1-D2.1/2 


\section{RÉSUMÉ DE LA LOGIQUE}

Demander le numéro de la personne qui désire enregistrer une feuille de temps.

Demander le nom de la personne qui désire enregistrer sa feuille de temps.

Demander le prénom de la personne qui désire enregistrer sa feuille de temps.

Inscrire le nom et le numéro de la personne dans le fichier de l'identification D2.1/1

Demander la date de la feuille de temps

Vérifier si la date de la feuille de temps est valide

sinon afficher message d'erreur

Enregistrer toutes les transactions

Pour chaque transaction

Enregistrer la date de la transaction

Valider la date de la transaction avec les coordonnées de la feuille de temps

Demander si l'activité est facturable

Si l'activité est facturable

Enregistrer l'identification du client

Afficher un à un les clients correspondants à l'identification fournie jusqu'a ce que

la personne indique que c'est le bon client

Si la personne n'a jamais indiqué que c'est le bon client

Indiquer que c'est un nouveau client

Demander le nom du client

Inscrire le nom dans le fichier maître client D2.1/2

Demandez le numéro de l'associé

Inscrire le numéro de l'associé dans le fichier maître client D2.1/2

Enregistrer le code d'activité

Vérifier si le code d'activité existe

Si le code existe

Si oui, affichez le nom de l'activité

Demander le temps passé à exécuter l'activité

Vérifiez si le temps est un multiple de .25

Enregistrez la description du travail effectué

sinon, affichez message d'erreur

Si l'activité n'est pas facturable

Enregistrez le code d'activité

Vérifiez si le code d'activité existe

Si le code existe

Si oui, affichez le nom de l'activité

Demandez le temps passé à exécutez l'activité

Vérifiez si le temps est un multiple de .25

Sinon, affichez message d'erreur

Vérifiez si le code d'activité signifie NON § L'EMPLOI

Si oui vérifiez que le temps de l'activité ne soit pas supérieur

à 7 heures.

Enregistrez la description du travail effectué 
Processus de traitement

\section{Imprimer feuille de temps, 2.1.2}

\section{DESCRIPTION}

Imprimer à partir des transactions contenues dans le fichier transactions, la feuille de temps pour un employé ou associé donné.

ENTRÉE

Nom, D2.1/1-2.1.2

Numéro, D2.1/1-2.1.2

Date de la feuille de temps, b-2.1.2

Transactions, D2.1/6-2.1.2

Coordonnées de la feuille de temps,

D2.1/3-2.1.2
SORTIE

Feuille de temps, 2.1.2-2.1.4

Feuille de temps, 2.1.2-2.1.3

Date de la feuille de temps, 2.1.2-D2.$1 / 3$ 


\section{RÉSUME DE LA LOGIQUE}

demander la date de la feuille de temps que l'on désire imprimer

Vérifiez si la date de la feuille de temps est valide

sinon affichez message d'erreur

Sur chaque page de feuille de temps

Imprimez le numéro de l'employé ou de l'associé

Imprimez le nom et le prénom de l'employé ou de l'associé

Imprimez la date de la feuille de temps

Imprimer le numéro de la page

Pour chaque journée incluse dans la feuille de temps

Pour chaque transaction correspondant à la journée incluse dans la feuille de temps Imprimez le numéro de la transaction

Imprimez le numéro du client

Imprimez le nom du client

Imprimez le code d'activité

Imprimez le temps

Si le code d'activité ne signifie pas que l'employé a donné ou suivi un cours

Additionnez le temps au temps de la journée

Imprimez la description du travail effectué

Imprimez le temps total pour la journée

Imprimez le temps supplémentaire de la journée

Changez de journée

Imprimez le temps de la semaine

Imprimez le temps supplémentaire de la semaine 
Processus de traitement

\title{
Corriger feuille de temps, 2.1.3
}

\author{
DESCRIPTION
}

Consiste à modifier une fiche du fichier transactions.

\section{ENTRÉES}

feuille de temps, 2.1.2-2.1.3

corrections, 2.1.4-2.1.3

numéro de la transaction, b-2.1.3

numéro, $02.1 / 2-2.1 .3$

nom du client, D2.1/2-2.1.3

transaction, D2.1/6-2.1.3

nom de l'activité, D2.1/5-2.1.3

\section{SORTIE}

transactions, 2.1.3-D2/6

code, D5-2.1.3

numéro, 2.1.3-D2.1/6

identification, 2.1.3-D2.1/2

transaction, 2.1.3-D2.1/6

\section{RÉSUMÉ DE LA LOGIQUE}

Demander le numéro de la transaction à corriger

Vérifiez s'il y a une transaction ayant le numéro demandé

Afficher le contenu de la transaction

Pour chaque champ de la transaction

Demander l'information à saisir (1)

si retour de chariot, conserver la même information

Imprimez la feuille de temps (voir processus 2.1.2) 
Processus de traitement

\section{Approuver feuilles de temps, 2.1.4}

\section{DESCRIPTION}

Vérifiez la répartition du temps entre chacune des activités.

ENTRÉES

feuille de temps, 2.1.2-2.1.4

\section{SORTIE}

feuille de temps, 2.1.4-D2.1/4

corrections, 2.1.4-2.1.3

RÉSUMÉ DE LA LOGIQUE

Regardez la feuille de temps

Si la feuille de temps est sans erreur, la signer sinon demandez correction 
Flux de données 
Flux de données

\title{
Données feuille de temps, $b-2.1 .1$
}

\author{
REF. SOURCE: b DESTINATION: Membre du personnel \\ REF. DESTIN.: $\quad 2.1 .1 \quad$ DESTINATION: Enregistrer données feuilles- \\ temps
}

\section{DESCRIPTION DÉTAILLÉE}

Les données de la feuille de temps représentent toute l'information relative à l'emploi du temps d'un employé ou associé. A chaque jour, chaque membre du personnel ( Associés et employés) note dans leur agenda les tâches effectuées et le temps qui leurs est consacré.

STRUCTURE DE DONNES INCLUSES:

date du jour: 2 caractères numériques

identification du client: 30 caractères alphanumériques

code de l'activité: 3 caractères numériques

description de l'activité: $\mathbf{4 0}$ caractères alphanumériques

temps: 4 caractères numériques ( 2 décimales)

VOLUME

Harel Drouin, et Associés $\quad: 35,280$

Harel Drouin Gestion Conseil $\quad$ : 5,880

Société d'informatique de gestion $: \underline{8,820}$

Total par semaine $\quad: \quad 49,980$ 
Flux de données

\section{Date feuille de temps, b-2.1.1}

$\begin{array}{llll}\text { REF. SOURCE: } & \text { b } & \text { DESCRIPTION: } & \text { Membre du personnel } \\ \text { REF. DESTIN.: } & 2.1 .1 & \text { DESCRIPTION: } & \text { Enregistrer données feuilles- } \\ & & & \text { temps }\end{array}$

\section{DESCRIPTION DÉTAILLÉE}

C'est la date de la feuille de temps pour laquelle l'usager désire imprimer des données. Le système permettra aux usagers d'enregistrer des données pour deux semaines distinctes, c'est pourquoi le système validera la date de la feuille de temps. A l'aide de cette date, le système validera les dates des transactions fournies.

Le volume a été calculé en fonction du nombre d'employés pour chacune des entreprises. Nous avons posé l'hypothèse que l'employé ou l'associé enregistre ses données de feuille de temps qu'une seule fois par semaine. Si tous les employés produisent deux feuilles de temps au cours de la même semaine, ou si les employé utilisent plus d'une séance pour produire leur feuille de temps, le volume doublera. Cependant, jusqu'a présent, les employés ou associés n'utilisent qu'une seule séance pour produire la feuille de temps.

STRUCTURE DE DONNÉES INCLUSES

date: 8 caractères alphanumériques

VOLUME

Harel Drouin, et Associés $\quad: 180$

Harel Drouin Gestion Conseil $\quad$ : 30

Société d'informatique de gestion : $\underline{45}$

Total par semaine $\quad: 255$ 
Flux de données

\section{Nom et numéro, b-2.1.1}

$\begin{array}{llll}\text { REF. SOURCE: } & b & \text { DESTINATION: } & \text { Membre du personnel } \\ \text { REF. DESTIN.: } & 2.1 .1 & \text { DESTINATION: } & \text { Enregistrer données feuilles- } \\ & & & \text { temps }\end{array}$

\section{DESCRIPTION DÉTAILLÉE}

Le numéro, le nom et le prénom des personnes qui désirent enregistrer les données nécessaires à la production de leur feuille de temps.

Les volumes ont été calculé en fonction du nombre d'employés pour chacune des entreprises. Nous avons posé l'hypothèse qu'un employé enregistre ses données de feuille de temps qu'une seule fois par semaine. Si tous les employés produisent deux feuilles de temps au cours de la même semaine, le volume doublera. La situation sera la même si l'employé utilise deux séances au cours de la même semaine pour enregistrer ses feuilles de temps. Cependant, jusqu'a présent, les employés n'utilise généralement qu'une seule séance pour produire la feuille de temps.

STRUCTURE DE DONNÉES INCLUSES

numéro: 3 caractères numériques

nom: 20 caractères alphanumériques

prénom: 20 caractères alphanumériques 
VOLUME

Harel Drouin, et Associés $\quad: 180$

Harel Drouin Gestion Conseil : $\quad 30$

Société d'informatique de gestion : 45

Total par semaine $\quad$ : 255 
Flux de données

\section{Nom du client, 2.1.2-D2.1/2}

REF. SOURCE: $\quad 2.1 .2$

REF. DESTIN.:
D2.1/2

DESCRIPTION:
Enregistrer données feuilles-

temps

Fichier maître client

DESCRIPTION DÉTAILLÉE

Ce flux est utilisé pour tous les nouveaux clients dont le nom n'apparaît pas dans le fichier client.

STRUCTURE DE DONNÉES INCLUSES

nom du client: maximum 60 caractères alphanumériques

VOLUME

Harel Drouin, et Associés $\quad: 2$

Harel Drouin Gestion Conseil $\quad: 2$

Société d'informatique de gestion $: \underline{2}$

Total par semaine $\quad: 6$ 
Flux de données

\section{Identification, 2.1.1-D2.1/2}

REF. SOURCE:

2.1.1

DESCRIPTION:

Enregistrer données feuillestemps

REF. DESTIN.:

D2.1/2

DESCRIPTION:

Fichier maître client

\section{DESCRIPTION DÉTAILLÉE}

Ce flux contient une chaîne de caractères permettant de retracer un client dans le fichier client.

STRUCTURE DE DONNÉES INCLUSES

identification: maximum 30 caractères alphanumériques

VOLUME

Harel Drouin, et Associés $\quad: 35,280$

Harel Drouin Gestion Conseil $\quad$ : 5,880

Société d'informatique de gestion $: \underline{8.820}$

Total par semaine $\quad: 49,980$ 
Flux de données

\section{Numéro, D2.1/2-2.1.1}

$\begin{array}{llll}\text { REF. SOURCE: } & \text { D2.1/2 } & \text { DESCRIPTION: } & \text { Fichier maître client } \\ \text { REF. DESTIN.: } & 2.1 .1 & \text { DESCRIPTION: } & \begin{array}{l}\text { Enregistrer données feuilles- } \\ \text { temps }\end{array}\end{array}$

\section{DESCRIPTION DÉTAILLÉE}

Le numéro d'identification du client correspondant à l'identification fournie par la personne qui désire produire sa feuille de temps.

STRUCTURE DE DONNÉES INCLUSES

numéro du client: 8 caractères numériques

VOLUME

Harel Drouin, et Associés $\quad: 35,280$

Harel Drouin Gestion Conseil $\quad$ : 5,880

Société d'informatique de gestion $: \underline{\mathbf{8 . 8 2 0}}$

Total par semaine $\quad: 49,980$ 
Flux de données

Nom, D2.1/2-2.1.1

$\begin{array}{llll}\text { REF. SOURCE: } & \text { D2.1/2 } & \text { DESCRIPTION: } & \text { Fichier maître client } \\ \text { REF. DESTIN.: } & 2.1 .1 & \text { DESCRIPTION: } & \text { Enregistrer données feuilles- } \\ & & & \text { temps }\end{array}$

\section{DESCRIPTION DÉTAILLÉE}

Le nom du client inscrit dans le fichier maître clients correspondant à l'identification fournie par la personne qui désire produire sa feuille de temps.

STRUCTURE DE DONNÉES INCLUSES

nom: 60 caractères alphanumériques

VOLUME

Harel Drouin, et Associés $\quad: 35,280$

Harel Drouin Gestion Conseil $\quad$ : 5,880

Société d'informatique de gestion : $\underline{8.820}$

Total par semaine $\quad: 49,980$ 
Flux de données

\section{Données feuille de temps, 2.1.1-D2.1/6}

REF. SOURCE

DESCRIPTION:

Enregistrer données feuillestemps

REF. DESTIN.

D2.1/6

DESCRIPTION:

Transactions feuilles de temps

\section{DESCRIPTION DÉTAILLÉE}

Les données de la feuille de temps représentent toute l'information relative à l'emploi d'un employé ou d'un associé. Ces données serviront à effectuer la mise à jour des travaux en cours.

\section{STRUCTURE DE DONNÉES INCLUSES}

numéro de la transaction: 5 caractères numériques

numéro de l'employé ou de l'associé: 3 caractères numériques

date de la transaction: 8 caractères alphanumériques

numéro du client: 8 caractères numériques

nom du client: 60 caractères alphanumériques

numéro de l'associé: 3 caractères numériques

code de l'activité: 3 caractères numériques

temps: 4 caractères numériques ( 2 décimales)

description de l'activité: $\mathbf{4 0}$ caractères alphanumériques 


\section{VOLUME}

Harel Drouin, et Associés : : 35,280

Harel Drouin Gestion Conseil : $\quad$ 5,880

Société d'informatique de gestion $: \underline{8,820}$

Total par semaine $\quad: 49,980$ 
Flux de données

\section{Code, 2.1.1-D2.1/5}

$\begin{array}{llll}\text { REF. SOURCE: } & 2.1 .1 & \text { DESCRIPTION: } & \begin{array}{l}\text { Enregistrer données feuilles- } \\ \text { temps }\end{array} \\ \text { REF. DESTIN.: } & \text { D2.1/5 } & \text { DESCRIPTION: } & \text { Codes d'activités }\end{array}$

\section{DESCRIPTION DÉTAILLÉE}

C'est un chiffre correspondant à l'activité effectuée par l'employé ou l'associé. Ce code permet d'identifier dans le fichier des codes d'activités, le nom de l'activité correspondant au code saisi.

STRUCTURE DE DONNÉES INCLUSES

code d'activité: 3 caractères numériques

VOLUME

Harel Drouin, et Associés $\quad: \quad 35,280$

Harel Drouin Gestion Conseil $\quad$ : 5,880

Société d'informatique de gestion $: \underline{8.820}$

Total par semaine $\quad: 49,980$ 
Flux de données

\section{Nom de l'activité, D2.1/5-2.1.1}

$\begin{array}{llll}\text { REF. SOURCE: } & \text { D2.1/5 } & \text { DESCRIPTION: } & \text { Codes d'activités } \\ \text { REF. DESTIN.: } & 2.1 .1 & \text { DESCRIPTION: } & \text { Enregistrer données feuilles- } \\ & & & \text { temps }\end{array}$

\section{DESCRIPTION DÉTAILLÉE}

A chaque fois qu'un employé ou associé enregistre une activité, il doit inscrire le code de l'activité. Avec ce code, le système lit dans la liste des codes d'activité le nom de l'activité correspondant au code fourni et l'affiche à l'écran.

STRUCTURE DE DONNÉES INCLUSES

nom: 60 caractères alphanumériques

$\begin{array}{ll}\text { VOLUME } & \\ \text { Harel Drouin, et Associés } & : 35,280 \\ \text { Harel Drouin Gestion Conseil } & : 5,880 \\ \text { Société d'informatique de gestion } & : \underline{8,820} \\ \text { Total par semaine } & : 49,980\end{array}$


Flux de données

Coordonnées de la feuille de temps, D2.1/3-2.1.1

$\begin{array}{llll}\text { REF. SOURCE: } & \text { D2.1/3 } & \text { DESCRIPTION: } & \text { Coordonnées feuilles de temps } \\ \text { REF. DESTIN.: } & 2.1 .1 & \text { DESCRIPTION: } & \text { Enregistrer données feuilles- } \\ & & \text { temps }\end{array}$

\section{DESCRIPTION DÉTAILLÉE}

Ce flux utilisé par les processus 2.1.1 Enregistrer données feuilles-temps. II sert à valider les dates de transactions fournies par les usagers.

\section{STRUCTURE DE DONNÉES INCLUSES}

date de la feuille de temps: 8 caractères alphanumériques

1 ère journée: 2 caractères alphanumériques

nombre d'heures régulières pour la première journée: 1 caractère numérique

2 ème journée: 2 caractères alphanumériques

nombre d'heures régulières pour la deuxième journée: 1 caractère numérique

3 ème journée: 2 caractères alphanumériques

nombre d'heures régulières pour la troisième journée: 1 caractère numérique

4 ème journée: 2 caractères alphanumériques

nombre d'heures régulières pour la quatrième journée: 1 caractère numérique

5 ème journée: 2 caractères alphanumériques

nombre d'heures régulières pour la cinquième journée: 1 caractère numérique

6 ème journée: 2 caractères alphanumériques

nombre d'heures régulières pour la sixième journée: 1 caractère numérique

7 ème journée: 2 caractères alphanumériques

nombre d'heures régulières pour la septième journée: 1 caractère numérique 
VOLUME

Harel Drouin, et Associés $\quad: 180$

Harel Drouin Gestion Conseil : 30

Société d'informatique de gestion : 45

Total par semaine $\quad$ : 255 
Flux de données

\section{Date de la feuille de temps, 2.1.1-D 2.1/3}

REF. SOURCE:

DESCRIPTION:

Enregistrer données feuillestemps

REF. DESTIN

D 2.1/3

DESCRIPTION:

Coordonnées de la feuille de temps

\section{DESCRIPTION DÉTAILLÉE}

C'est la date de la feuille de temps fournie par l'usager qui désire produire sa feuille de temps. Cette date permet de vérifier dans le fichier Coordonnes de la feuille de temps D2.1/3, s'il y a une feuille de temps qui doit être produite pour la date fournie. Elle permet également de connaître les dates des journées incluses dans la feuille de temps.

Le volume a été calculé en fonction du nombre d'employés pour chacune des entreprises. Nous avons posé l'hypothèse que l'employé ou l'associé enregistre ses données de feuille de temps qu'une seule fois par semaine. Si tous les employés produisent deux feuilles de temps au cours de la même semaine, ou si les employé utilisent plus d'une séance pour produire leur feuille de temps, le volume doublera. Cependant, jusqu'a présent, les employés ou associés n'utilisent qu'une seule séance pour produire la feuille de temps.

STRUCTURE DE DONNÉES INCLUSES

date: 8 caractères numériques 
VOLUME

Harel Drouin, et Associés $\quad: 180$

Harel Drouin Gestion Conseil : : 30

Société d'informatique de gestion : $\underline{45}$

Total par semaine $\quad$ : 255 
Flux de données

\section{Nom et numéro, 2.1.1-D2.2/1}

$\begin{array}{llll}\text { REF. SOURCE: } & 2.1 .1 & \text { DESCRIPTION: } & \begin{array}{l}\text { Enregistrer données feuilles- } \\ \text { temps }\end{array} \\ \text { REF. DESTIN.: } & \text { D2.2/1 } & \text { DESCRIPTION: } & \text { Identification }\end{array}$

\section{DESCRIPTION DÉTAILLÉE}

Ce flux contient le nom et le numéro de l'employé ou de l'associé qui désire compléter sa feuille de temps. Ce flux est utilisé seulement la première fois que le logiciel de feuille de temps sera utilisé.

STRUCTURE DE DONNÉES INCLUSES

numéro: 3 caractères numériques

nom: 20 caractères alphanumériques

prénom: 20 caractères alphanumériques

VOLUME

Harel Drouin, et Associés $\quad: 180$

Harel Drouin Gestion Conseil : 30

Société d'informatique de gestion : $\underline{45}$

Total par semaine $\quad$ : 255 
Flux de données

\section{Date de la feuille de temps, b-2.1.2}
REF. SOURCE:
b
DESCRIPTION:
Membre du personnel
REF. DESTIN.:
2.1.2
DESCRIPTION:
Imprimer feuilles de temps

\section{DESCRIPTION DÉTAILLÉE}

C'est la date de la feuille de temps pour laquelle l'usager désire imprimer une feuille de temps. Étant donné que le système doit prévoir la possibilité d'enregistrer des données pour deux feuilles de temps distinctes, une sélection des transactions doit être effectuée lors de l'impression. Pour calculer le volume, nous avons posé l'hypothèse que $50 \%$ des usagers impriment deux fois leur feuille de temps.

STRUCTURE DE DONNÉES INCLUSES

date: 8 caractères alphanumériques

VOLUME

Harel Drouin, et Associés $\quad: 270$

Harel Drouin Gestion Conseil : : 30

Société d'informatique de gestion : $\underline{45}$

Total par semaine $\quad: 255$ 
Flux de données

Nom, D2.1/1-2.1.2

REF. SOURCE:

D2.1/1

DESCRIPTION:

Identification

REF. DESTIN.:

2.1 .2

DESCRIPTION:

Imprimer feuille de temps

\section{DESCRIPTION}

Nom de l'employé ou de l'associé qui imprime sa feuille de temps.

Le volume a été calculé en fonction du nombre d'employés et associés pour chacune des entreprises. Nous avons posé l'hypothèse que $50 \%$ des employés ou associés impriment leurs feuilles de temps qu'une seule fois. Si tous les employés ou associés impriment deux feuilles de temps au cours de la même semaine, le volume augmentera d'environ $35 \%$.

STRUCTURE DE DONNÉES INCLUSES

nom: 20 caractères alphanumériques

prénom: 20 caractères alphanumériques

VOLUME

Harel Drouin, et Associés $\quad: 180$

Harel Drouin Gestion Conseil $\quad$ : 30

Société d'informatique de gestion : $\underline{45}$

Total par semaine $\quad$ : 255 
Flux de données

\section{Numéro, 2.1.2-2.1/1}

$\begin{array}{llll}\text { REF. SOURCE: } & 2.1 .2 & \text { DESCRIPTION: } & \text { Imprimer feuille de temps } \\ \text { REF. DESTIN.: } & 2.1 / 1 & \text { DESCRIPTION: } & \text { Identification }\end{array}$

\section{DESCRIPTION DÉTAILLÉE}

C'est le numéro de l'employé ou de l'associé qui imprime sa feuille de temps.

Le volume a été calculé en fonction du nombre d'employés et associés pour chacune des entreprises. Nous avons posé l'hypothèse que $50 \%$ des employés ou associés impriment leurs feuilles de temps qu'une seule fois. Si tous les employés ou associés impriment deux feuilles de temps au cours de la même semaine, le volume augmentera d'environ $35 \%$.

\section{STRUCTURE DE DONNÉES INCLUSES}

numéro: 3 caractères numériques

\section{VOLUME}

Harel Drouin, et Associés $\quad: 270^{(1)}$

Harel Drouin Gestion Conseil : 45

Société d'informatique de gestion : 68

Total par semaine 
Flux de données

\title{
Date de la feuille de temps, 2.1.2-D2.1/3
}

\author{
REF. SOURCE: 2.1.2 DESCRIPTION: Imprimer feuille de temps \\ REF. DESTIN.: D2.1/3 DESCRIPTION: Coordonnées feuille de temps
}

DESCRIPTION DÉTAILLÉE

Ce flux contient la date de la feuille temps pour laquelle on désire imprimer la feuille de temps. La date de la feuille de temps set à aller valider la date fournie et à aller chercher les journées incluses dans cette semaines de feuilles de temps.

STRUCTURE DE DONNÉES INCLUSES

date de la feuille de temps: 8 caractères alphanumériques

VOLUME

Harel Drouin, et Associés $\quad: 180$

Harel Drouin Gestion Conseil : : 30

Société d'informatique de gestion : $\underline{45}$

Total par semaine $\quad: 255$ 
Flux de données

\section{Coordonnées de la feuille de temps, D2.1/3-2.1.2}
REF. SOURCE:
D2.1/3
DESCRIPTION:
Coordonnées feuille de temps
REF. DESTIN.:
2.1.2
DESCRIPTION:
Imprimer feuille de temps

\section{DESCRIPTION DÉTAILLÉE}

Ce flux contient la date de la feuille de temps et le nombre d'heures pour chacune des journées incluses dans la feuille de temps. Ce flux est principalement utilisé dans le but de calculer le temps supplémentaire.

STRUCTURE DE DONNÉES INCLUSES

date de la feuille de temps: 8 caractères alphanumériques

1 ère journée: 2 caractères alphanumériques

nombre d'heures régulières pour la première journée: 1 caractère numérique

2 ème journée: 2 caractères alphanumériques

nombre d'heures régulières pour la deuxième journée: 1 caractère numérique

3 ème journée: 2 caractères alphanumériques

nombre d'heures régulières pour la troisième journée: 1 caractère numérique

4 ème journée: 2 caractères alphanumériques

nombre d'heures régulières pour la quatrième journée: 1 caractère numérique

5 ème journée: 2 caractères alphanumériques

nombre d'heures régulières pour la cinquième journée: 1 caractère numérique

6 ème journée: 2 caractères alphanumériques

nombre d'heures régulières pour la sixième journée: 1 caractère numérique

7 ème journée: 2 caractères alphanumériques

nombre d'heures régulières pour la septième journée: 1 caractère numérique

feuille complétée: 1 caractère alphanumérique 


\begin{abstract}
VOLUME
Harel Drouin, et Associés $\quad$ : 270

Harel Drouin Gestion Conseil : : 30

Société d'informatique de gestion : $\underline{45}$

Total par semaine $\quad$ : 255
\end{abstract}


Flux de données

\title{
Feuille de temps, 2.1.2-2.1.3
}

\author{
REF. SOURCE: 2.1.2 DESCRIPTION: Imprimer feuille de temps \\ $\begin{array}{llll}\text { REF. DESTIN.: } & 2.1 .3 & \text { DESCRIPTION: } & \text { Corriger feuille de temps }\end{array}$
}

\section{DESCRIPTION DÉTAILLÉE}

Contient la description de l'emploi du temps pour un employé ou un associé.

\section{STRUCTURE DE DONNÉES INCLUSES}

nom de l'employé ou de l'associé: 20 caractères alphanumériques

prénom de l'employé ou de l'associé: 20 caractères alphanumériques

no de l'employé ou de l'associé: 3 caractères numériques

\section{Pour chaque transaction:}

numéro de la transaction: 5 caractères numériques

numéro du client: 8 caractères numériques

nom du client: 60 caractères alphanumériques

numéro de l'associé: 3 caractères numériques

code de l'activité: 3 caractères numériques

temps: 4 caractères numériques ( 2 décimales)

taux horaire: 3 caractères numériques

description de l'activité: $\mathbf{4 0}$ caractères alphanumériques

Pour chaque journée:

total de la journée: 4 caractères numériques dont deux décimales temps supplémentaire de la journée: 4 caractères numériques dont deux décimales 
Pour la feuille de temps:

total de la semaine: 4 caractères numériques dont deux décimales

temps supplémentaire de la semaine: 4 caractères numériques dont deux décimales

VOLUME

Harel Drouin, et Associés $\quad: 180$

Harel Drouin Gestion Conseil $\quad$ : 30

Société d'informatique de gestion : $\underline{45}$

Total par semaine $\quad$ : 255 
Flux de données

\section{Feuille de temps, 2.1.2-2.1.4}

$\begin{array}{llll}\text { REF. SOURCE: } & 2.1 .2 & \text { DESCRIPTION: } & \text { Imprimer feuille de temps } \\ \text { REF. DESTIN.: } & 2.1 .4 & \text { DESCRIPTION: } & \text { Approuver feuille de temps }\end{array}$

\section{DESCRIPTION DÉTAILLÉE}

Contient la description de l'emploi du temps pour un employé ou un associé.

\section{STRUCTURE DE DONNÉES INCLUSES}

nom de l'employé ou de l'associé: $\mathbf{2 0}$ caractères numériques

prénom de l'employé ou de l'associé: 20 caractères numériques

no de l'employé ou de l'associé: 3 caractères numériques

Pour chaque transaction:

numéro de la transaction: 5 caractères numériques

numéro du client: 8 caractères numériques

nom du client: 60 caractères alphanumériques

numéro de l'associé: 3 caractères numériques

code de l'activité: 3 caractères numériques

temps: 4 caractères numériques ( 2 décimales)

description de l'activité: $\mathbf{4 0}$ caractères alphanumériques

Pour chaque journée:

total de la journée: 4 caractères numériques dont deux décimales temps supplémentaire de la journée: 4 caractères numériques dont deux décimales Pour la feuille de temps:

total de la semaine: 5 caractères numériques dont deux décimales temps supplémentaire de la semaine: 4 caractères numériques dont deux décimales 


\begin{abstract}
VOLUME
Harel Drouin, et Associés $\quad: 180$

Harel Drouin Gestion Conseil : $\quad 30$

Société d'informatique de gestion : 45

Total par semaine $\quad$ : 255
\end{abstract}


Flux de données

\section{Transactions, D2.1/6-2.1.2}

$\begin{array}{llll}\text { REF. SOURCE: } & \text { D2.1/6 } & \text { DESCRIPTION: } & \text { Transactions feuille de temps } \\ \text { REF. DESTIN.: } & 2.1 .2 & \text { DESCRIPTION: } & \text { Imprimer feuilles de temps }\end{array}$

DESCRIPTION DÉTAILLÉE

Contient linformation relative à l'emploi d'un employé ou d'un associé. Chacune des transactions est utilisée pour imprimer la feuille de temps.

STRUCTURE DE DONNÉES INCLUSES

numéro de la transaction: 5 caractères numériques

numéro de l'employé ou de l'associé: 3 caractères numériques

date de la transaction: 8 caractères alphanumériques

numéro du client: 8 caractères numériques

nom du client: 60 caractères alphanumériques

numéro de l'associé: 3 caractères numériques

code de l'activité: 3 caractères numériques

temps: 4 caractères numériques ( 2 décimales)

description de l'activité: 40 caractères alphanumériques

\section{VOLUME}

Harel Drouin, et Associés $\quad: 52,920$

Harel Drouin Gestion Conseil $\quad$ : $\quad 8,820$

Société d'informatique de gestion $: \underline{13,230}$

Total par semaine $\quad: \mathbf{7 4 , 2 4 0}$ 


\section{HYPOTHĖSE}

4 transactions par heures

7 heures par jour

7 jours par semaines

180 employés

$50 \%$ des employé impriment 2 fois -> 17640

TOTAL $\quad 52920$ 
Flux de données

\section{Transaction, 2.1.3-D2.1/6}

$\begin{array}{llll}\text { REF. SOURCE: } & 2.1 .3 & \text { DESCRIPTION: } & \text { Corriger feuille de feuille } \\ \text { REF. DESTIN.: } & \text { D2.1/6 } & \text { DESCRIPTION: } & \text { Transactions feuilles de temps }\end{array}$

\section{DESCRIPTION DÉTAILLÉE}

Une transaction nous informe du travail effectué dans un dossier par un associé ou un employé et ce pour une période donnée. Une transaction contient un numéro d'identification, le numéro de l'employé concerné par la transaction, la date de la transaction, le numéro du client, le code de l'activité exécutée, le temps passé à exécuter l'activité et la description narrative des tâches effectuées.

Le volume a été calculé en fonction du nombre d'employés et associés pour chacune des entreprises. Nous avons posé l'hypothèse qu'au maximum 10\% des transactions seront corrigées. 


\section{STRUCTURE DE DONNÉES INCLUSES}

numéro de la transaction: 5 caractères numériques

numéro de l'employé ou de l'associé: 3 caractères numériques

date de la transaction: 8 caractères alphanumériques

numéro de l'associé: 3 caractères numériques

numéro du client: 8 caractères numériques

nom du client: 60 caractères alphanumériques

code de l'activité: 3 caractères numériques

temps: 4 caractères numériques ( 2 décimales)

taux horaire: 3 caractères numériques

description de l'activité: $\mathbf{4 0}$ caractères alphanumériques

\section{VOLUME}

Harel Drouin, et Associés $\quad: 3,528$

Harel Drouin Gestion Conseil $\quad$ : 588

Société d'informatique de gestion : $\mathbf{8 8 2}$

Total par semaine $\quad: 4,998$ 
Flux de données

\section{Numéro, 2.1.3-D2.1/6}

$\begin{array}{llll}\text { REF. SOURCE: } & 2.1 .3 & \text { DESCRIPTION: } & \text { Corriger feuille de temps } \\ \text { REF. DESTIN.: } & \text { D2.1/6 } & \text { DESCRIPTION: } & \text { Transactions feuille de temps }\end{array}$

DESCRIPTION DÉTAILLÉE

Ce flux contient le numéro de la transaction que l'on désire corriger. A l'aide de ce numéro, nous allons chercher la transaction dans le fichier des feuilles de temps D2.1/6.

STRUCTURE DE DONNÉES INCLUSES

numéro de la transaction: 5 caractères numériques

VOLUME

Harel Drouin, et Associés $\quad: 35,280$

Harel Drouin Gestion Conseil $\quad$ : 5,880

Société d'informatique de gestion $: \underline{8,820}$

Total par semaine $\quad: 49,980$ 
Flux de données

\section{Numéro, D2.1/2-2.1.3}

REF. SOURCE:

REF. DESTIN.:
D2.1/2
DESCRIPTION: DESCRIPTION:
Fichier maître client

Corriger feuille de temps

\section{DESCRIPTION DÉTAILLÉE}

Le numéro d'identification du client correspondant à l'identification fournie par la personne qui désire corriger sa feuille de temps.

Le volume a été calculé en fonction du nombre d'employés et associés pour chacune des entreprises. Nous avons posé l'hypothèse qu'au maximum 10\% des transactions saisies seront corrigées.

STRUCTURE DE DONNÉES INCLUSES

numéro du client: 8 caractères numériques

\section{VOLUME}

Harel Drouin, et Associés $\quad: 3,528$

Harel Drouin Gestion Conseil : 588

Société d'informatique de gestion : 882

Total par semaine $\quad: 4,998$ 
Flux de données

\section{Nom du client, D2.1/2-2.1.3}

REF. SOURCE:

D2.1/2

DESCRIPTION:

Fichier maître client

REF. DESTIN.:

2.1.3

DESCRIPTION:

Corriger feuille de temps

\section{DESCRIPTION DÉTAILLÉE}

Le nom du client inscrit dans le fichier maître client correspondant à lidentification fournie.

Le volume a été calculé en fonction du nombre d'employés et associés pour chacune des entreprises. Nous avons posé l'hypothèse qu'au maximum 10\% des transactions saisies seront corrigées.

STRUCTURE DE DONNÉES INCLUSES

nom: 60 caractères alphanumériques

\section{VOLUME}

Harel Drouin, et Associés $\quad: 3,528$

Harel Drouin Gestion Conseil $\quad$ : 588

Société d'informatique de gestion : $\quad \underline{882}$

Total par semaine $\quad: 4,998$ 
Flux de données

\section{Identification, 2.1.3-D2.1/2}

$\begin{array}{llll}\text { REF. SOURCE: } & 2.1 .3 & \text { DESCRIPTION: } & \text { Corriger feuille de temps } \\ \text { REF. DESTIN.: } & \text { D2.1/2 } & \text { DESCRIPTION: } & \text { Fichier maitre client }\end{array}$

DESCRIPTION DÉTAILLÉE

Ce flux contient une chaîne de caractères permettant de retracer un client dans le fichier client.

STRUCTURE DE DONNÉES INCLUSES

identification: maximum 30 caractères alphanumériques

$\begin{array}{ll}\text { VOLUME } & \\ \text { Harel Drouin, et Associés } & : 360^{(1)} \\ \text { Harel Drouin Gestion Conseil } & : 90 \\ \text { Société d'informatique de gestion } & : \underline{136} \\ \text { Total par semaine } & : 586\end{array}$


Flux de données

\title{
Numéro de la transaction, b-2.1.3
}

\author{
REF. SOURCE: $\quad b \quad$ DESCRIPTION: Membre du personnel \\ $\begin{array}{llll}\text { REF. DESTIN.: } & \text { 2.1.3 } & \text { DESCRIPTION: } & \text { Corriger feuille de temps }\end{array}$
}

\section{DESCRIPTION DÉTAILLÉE}

C'est le numéro identifiant la transaction à corriger. Les données de la feuille de temps enregistrées lors du processus 2.1.1 génèrent des transactions qui sont numérotées et enregistrées dans le fichier D2.1/6 transactions feuille de temps. Ce numéro est inscrit sur la feuille de temps lors de l'impression des transactions.

Le volume a été calculé en fonction du nombre d'employés et associés pour chacune des entreprises. Nous avons posé l'hypothèse que $50 \%$ des employés ou associés effectuent des corrections et qu'ils corrigent environ deux transactions par feuille de temps.

STRUCTURE DE DONNÉES INCLUSES

numéro de la transaction: 5 caractères numériques

VOLUME

Harel Drouin, et Associés $\quad: 180$

Harel Drouin Gestion Conseil : 30

Société d'informatique de gestion : $\underline{45}$

Total par semaine $\quad: 255$ 
Flux de données

\title{
Nom de l'activité, D2.1/5-2.1.3
}

\author{
REF. SOURCE: D2.1/5 DESCRIPTION: Codes d'activités \\ REF. DESTIN.: \\ DESCRIPTION: \\ Corriger la feuille de temps
}

DESCRIPTION DÉTAILLÉE

C'est le nom de l'activité correspondant au numéro de code d'activité inscrit dans la transaction ou le nom de l'activité correspondant au nouveau code enregistré.

Le volume a été calculé en fonction du nombre d'employés et associés pour chacune des entreprises. Nous avons posé l'hypothèse que $50 \%$ des employés ou associés effectuent des corrections et qu'ils corrigent environ deux transactions par feuille de temps.

STRUCTURE DE DONNÉES INCLUSES

nom: 60 caractères alphanumériques

\section{VOLUME}

Harel Drouin, et Associés $\quad: 180$

Harel Drouin Gestion Conseil : $\quad 30$

Société d'informatique de gestion : $\underline{45}$

Total par semaine $\quad$ : 255 
Flux de données

\section{Code, 2.1.3-D2.1/5}

$\begin{array}{llll}\text { REF. SOURCE: } & 2.1 .3 & \text { DESCRIPTION: } & \text { Corriger feuille de temps } \\ \text { REF. DESTIN.: } & \text { D2.1/5 } & \text { DESCRIPTION: } & \text { Codes d'activités }\end{array}$

DESCRIPTION DÉTAILLÉE

Code corrigé de l'activité décrivant le travail effectué.

STRUCTURE DE DONNÉES INCLUSES

code d'activité: 3 caractères numériques

VOLUME

Harel Drouin, et Associés $\quad: 3,528$

Harel Drouin Gestion Conseil : 588

Société d'informatique de gestion : 882

Total par semaine $\quad: 4,998$ 
Flux de données

\section{Corrections, 2.1.4-2.1.3}

REF. SOURCE:

2.1.4

DESCRIPTION:

Approuver feuille de temps

REF. DESTIN.:

DESCRIPTION:

Corriger feuille de temps

\section{DESCRIPTION DÉTAILLÉE}

La majorité des corrections effectuées concernent le temps passé à exécuter une activité. Occasionnellement, les corrections concerne un numéro de client.

STRUCTURE DE DONNÉES INCLUSES

temps: 4 caractères numériques ( 2 décimales)

VOLUME

Harel Drouin, et Associés $\quad: 360^{(1)}$

Harel Drouin Gestion Conseil $\quad$ : 90

Société d'informatique de gestion : 136

Total par semaine

: 586 
Flux de données

\section{Feuilles de temps, 2.1.4-D2.1/4}
REF. SOURCE:
2.1.4
DESCRIPTION:
Approuver feuilles de temps
REF. DESTIN.:
D2.1/4
DESCRIPTION:
Feuilles de temps

\section{DESCRIPTION DÉTAILLÉE}

Contient la description de l'emploi du temps pour un employé ou un associé.

\section{STRUCTURE DE DONNÉES INCLUSES}

nom de l'employé ou de l'associé: 20 caractères alphanumériques

prénom de l'employé ou de l'associé: 20 caractères alphanumériques

\section{Pour chaque transaction:}

numéro de la transaction: 5 caractères numériques

numéro du client: 8 caractères numériques

nom du client: 60 caractères alphanumériques

numéro de l'associé: 3 caractères numériques

code de l'activité: 3 caractères numériques

temps: 4 caractères numériques ( 2 décimales)

description de l'activité: 40 caractères alphanumériques

Pour chaque journée:

total de la journée: 4 caractères numériques dont deux décimales

temps supplémentaire de la journée: 4 caractères numériques dont deux décimales

\section{Pour la feuille de temps:}

total de la semaine: 4 caractères numériques dont deux décimales

temps supplémentaire de la semaine: 4 caractères numériques dont deux décimales 
VOLUME

Harel Drouin, et Associés $\quad: 180$

Harel Drouin Gestion Conseil : 30

Société d'informatique de gestion : 45

Total par semaine $\quad$ : 255 
Flux de données

$$
\text { \# ass, 2.1.1-D2.1/2 }
$$

REF. SOURCE:

DESCRIPTION:

Enregistrer données feuillestemps

REF. DESTIN.:

D2.1/2

DESCRIPTION:

Fichier maître client

\title{
DESCRIPTION DÉTAILLÉE
}

Ce flux contient le numéro de l'associé auquel appartient le client. Ce numéro est inscrit dans le fichier seulement lorsqu'il s'agit de nouveau client.

STRUCTURE DE DONNÉES INCLUSES

numéro de l'associé: 3 caractères numériques

\author{
VOLUME \\ Harel Drouin, et Associés $\quad: 2$ \\ Harel Drouin Gestion Conseil : : 2 \\ Société d'informatique de gestion : $\underline{2}$ \\ Total par semaine $\quad$ : 6
}


Flux de données

\# ass, D2.1/2-2.1.3

REF. SOURCE: D2.1/2 DESCRIPTION: Fichier maître client

REF. DESTIN.: $\quad 2.1 .3 \quad$ DESCRIPTION: Corriger feuilles de temps

DESCRIPTION DÉTAILLÉE

Ce flux contient le numéro de l'associé auquel appartient le client. Ce numéro est inscrit dans le fichier seulement lorsqu'il s'agit de nouveau client.

STRUCTURE DE DONNÉES INCLUSES

numéro de l'associé: 3 caractères numériques

VOLUME

Harel Drouin, et Associés $\quad: 35,280$

Harel Drouin Gestion Conseil $\quad$ : $\quad 5,880$

Société d'informatique de gestion $: \underline{8.820}$

Total par semaine $\quad: 49,980$ 
Processus 2.6, Produire rapports statistiques 
/ouvert.

Période Taux Heures

Heures Montants
chargeables $\$$

Déboursés

$\begin{array}{lllll} & & & \\ \text { Secret. Admin. } & \text { Bureau Repré. } & \text { MEURES NON-CHARGEABLES } \\ \text { Cours Vacances Maladie }\end{array}$

Heures Heures Hrs.Ch. Hrs.Ch.

** EQUIPE NO: 2 **

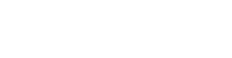

118 Deslande Guy
480 Mayer Pierre

$\begin{array}{ll}07 / 1988 & 29.0 b \\ 07 / 1988 & 79.00\end{array}$

0.00
167.50

$\begin{array}{cc}0.00 & 0.00 \\ 13232.50 & 0.00\end{array}$

0.00

** sous-TOtal **

263.50

$13232.50 \quad 0.00$

$\begin{array}{llllll}0.00 & 0.00 & 0.00 & 0.00 & 0.00 & 0.00 \\ 0.00 & 0.00 & 0.00 & 4.00 & 2.00 & 0.00\end{array}$

$\begin{array}{ll}0.00 & 0.00 \\ 7.00 & 7.00\end{array}$

$\begin{array}{rrrrrr}7.00 & 0.00 & 0.00 & 0.00 & -79.25 & 0.00 \\ & 7.00 & 0.00 & 20.00 & 40.50 & 187.50\end{array}$

$\begin{array}{llllll}7.00 & 7.00 & 0.00 & 20.00 & -38.75 & 187.50\end{array}$

Ce rapport peut prendre plusieurs formes, l'important c'est de retrouver cette information dans un ou plusieurs rapports. 
Pour la période: 07/1988

* EQUIPE NO: 3, JEAN DUHAMEL **

Monbre d'employés (a)

Montant $\$(c)$

Moyerne mensuelle - heures (b/a)

Moyenne mensuelle - s

Taux moyen $(c / a)$

Jours anrables $(h / 7)$

Productivité (b/h)

Temps secrétariat

Temps actministration

Temps bureau

Temps représentation

Temps cours-conférence

Temps vacances

Temps maladie

Temps congé

Temps total non-chargeable

6.32

595.75

3,026.25

$3,643.39$

4.00

147.00

0.00

5.00

5.00

$-186.00$ 
Harel, Drouin \& Associés

Heures des employés par mois (vacances exclues)

Page: 001

Date: $30 / 08 / 88$

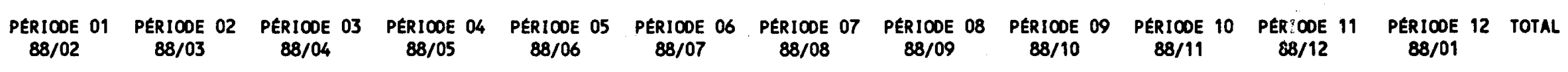

EQUIPE NO: 5, ROBERT MCLAUGHLIN

Employé no: 167 Oupuis Pierre

Employé no: 482 McLaughl in Robert

Employé no: 571 Payette Andre

TOTAL EQUIPE NO:5

$\begin{array}{rrrrrrrrrrrrr}147.00 & 161.00 & 147.00 & 154.00 & 119.00 & 147.00 & 0.00 & 0.00 & 0.00 & 0.00 & 0.00 & 0.00 & 875.00 \\ 141.75 & 231.00 & 192.25 & 193.75 & 193.50 & 87.25 & 0.00 & 0.00 & 0.00 & 0.00 & 0.00 & 0.00 & 1039.50 \\ 0.00 & 0.00 & 0.00 & 0.00 & 202.00 & 149.00 & 0.00 & 0.00 & 0.00 & 0.00 & 0.00 & 0.00 & 351.00 \\ \overline{288.75} & \overline{392.00} & \overline{339.25} & \overline{347.75} & \overline{514.50} & \overline{383.25} & \overline{0.00} & \overline{0.00} & \overline{0.00} & \overline{0.00} & \overline{0.00} & \overline{0.00} & \overline{2265.00}\end{array}$


Harel, Drouin \& Associés

Honoraires des employés par mois

Page: 001

Date: $30 / 08 / 88$ TAUX PERIOOE 01 PERIODE 02 PERIO0E 03 PER 88/03 $88 / 04$

PÉRIOOE 04

PERIOOE 05

PERIOOE O6

(2)

EOUIPE NO: 5, ROBERT MCLAUGHLIN

Employé no: 167 Dupuis Pierre

$93.00 \quad 13671.00 \quad 14973.00 \quad 13671.00$

Employé no: 482 McLaughl in Robert

$121.00 \quad 17151.75$

$27951.00 \quad 23262.25$

$14323.00 \quad 11067.00$ $88 / 07$

PERIOOE O7

PERIOOE 08

PERIOOE 09

PERIOOE 10

PERIODE 11 PERIODE 12 TOTAL

Employé no: 571 Payette André

59.00

$\begin{array}{llll}23262.25 & 23443.75 & 23413.50 & 10557.25\end{array}$

0.00

$\begin{array}{ll}0.00 & 0.00 \\ 0.00 & 0.00\end{array}$

0.00

0.00

$0.00 \quad 81373.00$

TOTAL EQUIPE:5

$30822.75 \quad 42924.00 \quad 36933.25$

$\frac{0.00}{37765.75} \frac{11918.00}{46398.50} \frac{8791.00}{33019.25}$

0.00

$\underline{0.00}$

- 0.00

0.00

$0.00 \quad 125779.50$

(1)

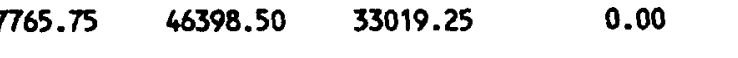

0.00

$0.00 \quad 29709.00$

$0.00 \quad 227863.50$ 
Harel, Drouin \& associés

Page: 001

Date: $30 / 08 / 88$

Rapport d'honoraires par mois/équipe

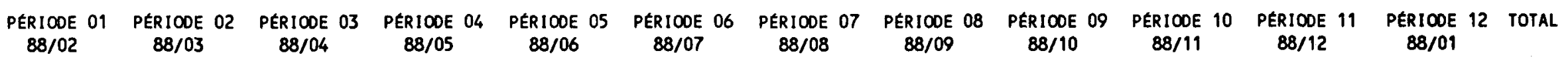

EQUIPE NO: 5, ROBERT MCLAUGHLIN

$\begin{array}{lrrrrrrrrrrrrr}\text { Heures chargeables budget (1) } & 182 & 182 & 192 & 164 & 182 & 128 & 146 & 162 & 164 & 192 & 146 & 182 & 2042 \\ \text { Heures chargeables réel (2) } & 194 & 245 & 237 & 191 & 252 & 241 & 0 & 0 & 0 & 0 & 0 & 0 & 1360 \\ \text { Heures chargeables année précédente (3) } & 1860 & 1860 & 1860 & 1860 & 1860 & 1860 & 1860 & 1860 & 1860 & 1860 & 1860 & 1860 & 22230 \\ \text { Honoraires budget } & 18200 & 18200 & 19110 & 16380 & 18200 & 12740 & 14560 & 12740 & 14560 & 19565 & 20544 & 15652 & 210325 \\ \text { Honoraires réels (4) } & 20313 & 26283 & 25547 & 20514 & 22382 & 20991 & 0 & 0 & 0 & 0 & 0 & 0 & 136030 \\ \text { Honoraires années précédentes (5) } & 80000 & 80000 & 80000 & 80000 & 80000 & 80000 & 80000 & 80000 & 80000 & 80000 & 80000 & 80000 & 360000 \\ \text { Taux moyen budget (6) } & 100 & 100 & 100 & 100 & 100 & 100 & 100 & 108 & 107 & 107 & 107 & 100 & 103\end{array}$

1- Le budget de tous les employés de l'équipe est total isé

1- Le budget de tous les employés de l''équipe
2- Total des heures facturables pour l'equipe

2- Total des heures facturables pour
3- Selon honoraires / taux moyen

4- Selon total des honoraires employés de l'équipe

5- Selon mise à jour honoraires année précédente

6. Honoraires budget / Heures chargeables budget 
Page: 002

Date: $30 / 08 / 88$

EOUIPE NO: 5, ROBERT MCLAUGHLIN

Taux moyen réel (7)

Taux moyen année précédente (8)

Heures chargeables/employé budget

Heures chargeables/employé réel

Heures chargeable/employé année précéd.

Nombre employés budget

Nombre enployés réel

Nombre employés années précédente
Harel, Drouin \& associés

Rapport d'honoraires par mois/équipe (suite)

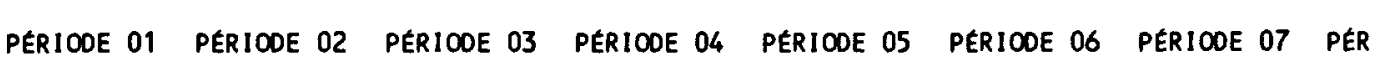
$88 / 02 \quad 88 / 03 \quad 88 / 04$ $88 / 05$ $\begin{array}{rr}\text { PERIOOE O6 } & \text { PERIOOE } \\ 88 / 07 & 88 / 08\end{array}$

$88 / 09$
PERIOOE 09 $88 / 10$
PÉRIOOE 10 PÉRIODE 11 $88 / 11$ PERIOOE 12 TOTAL
$88 / 01$

7- Honoraires / heures chargeables
8- Honoraires année précédente/ heures chargeables année précédente

$\begin{array}{rrrrrrrrr}105 & 107 & 108 & 107 & 89 & 87 & 0 & 0 & \\ 43 & 43 & 43 & 43 & 43 & 43 & 43 & 43 & \\ 147 & 161 & 147 & 155 & 154 & 147 & 160 & 154 & 146 \\ 146 & 100 & 118 & 96 & 82 & 95 & 0 & 0 & \\ 305 & 305 & 305 & 305 & 305 & 305 & 305 & 305 & \\ 1.24 & 1.13 & 1.31 & 1.06 & 1.18 & 0.87 & 0.91 & 1.18 & 1.12 \\ 1.33 & 2.44 & 2.01 & 2.00 & 3.06 & 2.53 & 0.00 & 0.00 & 0.00 \\ 6.10 & 6.10 & 6.10 & 6.10 & 6.10 & 6.10 & 6.10 & 6.10 & 6.10\end{array}$

0
43
154
0
305
1.25
0.00
6.10

43
160
305
0.91
0.00
6.10

\begin{tabular}{rr}
0 & 100 \\
43 & 43 \\
147 & 152 \\
0 & 102 \\
305 & 305 \\
\hline 1.24 & 13.40 \\
0.00 & 13.37 \\
6.10 & 73.20
\end{tabular}


Harel, Drouin \& Associés Sommaire d'honoraires par équipe

Page: 001

Date: $30 / 08 / 88$

** Pour la periode: 07/1988

Equipe chef d'équipe

$1 \quad$ Normand Verne

TOTAL

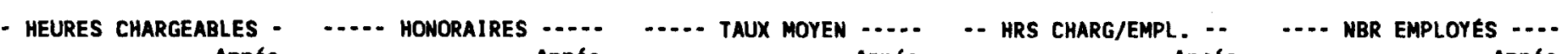

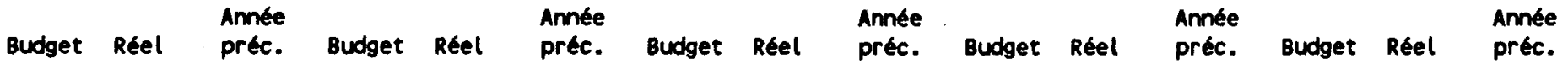

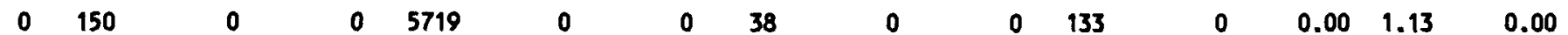

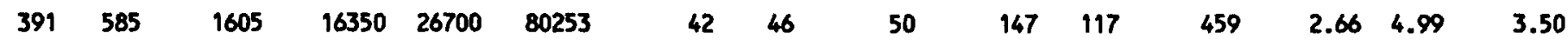

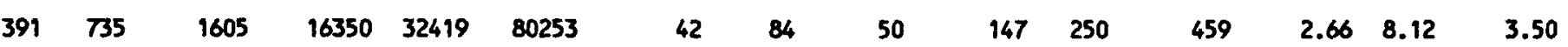


Page: 001

Date: $30 / 08 / 88$

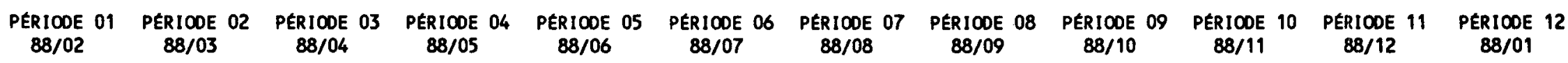

EQUIPE NO: 5, ROBERT MCLAUGHLIN

Employé no: 167 Dupuis Pierre

\begin{tabular}{|c|c|c|c|c|c|c|c|c|c|c|c|}
\hline 750.62 & .740 .57 & . 730.65 & $0.60 \quad 0.53$ & 0.440 .59 & 0.680 .43 & $0.00 \quad 0.45$ & $0.00 \quad 0.59$ & 0.000 .56 & $0.00 \quad 0.62$ & $0.00 \quad 0.45$ & $0.00 \quad 0.62$ \\
\hline 560.6 & 0.780 .57 & $0.87 \quad 0.65$ & 0.640 .53 & $0.55 \quad 0.59$ & 0.370 .43 & $0.00 \quad 0.45$ & $0.00 \quad 0.59$ & $0.00 \quad 0.56$ & $0.00 \quad 0.62$ & $0.00 \quad 0.45$ & $\begin{array}{lll}0.00 & 0.62\end{array}$ \\
\hline 00 & . & .000 .00 & .000 .00 & .640 .00 & .590 .00 & 0.000 .00 & 3.000 .00 & 0.000 .00 & $0.00 \quad 0.00$ & $0.00 \quad 0.00$ & \\
\hline
\end{tabular}

Employé no:571 Payette André 
Harel, Drouin et associés

Date: $30 / 08 / 88$

MONTANT

PERIOOE 01 PERIOOE 02 PERIOOE 03 PERIOOE O4 PERIOOE 05 PERIODE 06 PERIDOE

$\begin{array}{lllllll}88 / 02 & 88 / 03 & 88 / 04 & 88 / 05 & 88 / 06 & 88 / 07 & 88 / 08\end{array}$

Employe no: 167 Dupuis Pierre

330.12

Employe no:482 McLaughlin Robert

300.00

452.00

100.00

125.00

236.48

450.00

125.00

356.00

348.00

256.48

256.14

689.00

125.00
245.04

321.00

0.00

0.00

$258.69 \quad 0.00$

0.00

PERIODE 10
$88 / 11$

PÉRIODE 11

PERIOOE 12

CUMULATIF

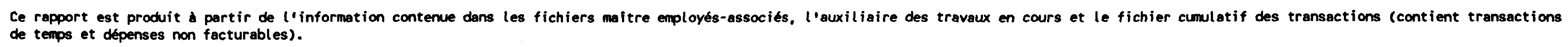


Harel, Drouin \& associés

Distribution cumulative du temps par employés

PAGE : 001

167 Dupuis Pierre

$$
\text { au 31/12/1989 }
$$

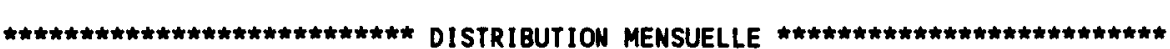

DÉBOURSES

Code Description

001 Restaurent

HEURES FACTURABLES

HEURES

$$
\begin{aligned}
& \text { MONTANT } \\
& \text { REEL }
\end{aligned}
$$

Code Description

160 Impôt

TOTAL HEURES FACTURABLES
$73.75 \quad 100.00 \quad 5236.25 \quad 100.00$

$\begin{array}{llll}73.75 & 47.10 & 5236.25 & 100.00\end{array}$
HEURES
BUDGET

$x$

$\begin{array}{lll}0.00 & 100.00 \quad 0.00\end{array}$

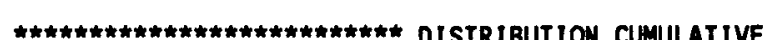

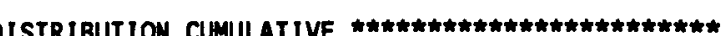

$0.00 \quad 100.00-0.00$

MONTANT

BUDGET

$x$

REEL

MONTANT

REEL

* HEURES

$x$

MONTANT $x$

BUDGET

$625.75 \quad 100.00$

41158.25

100.10

0.00

$0.00 \quad 0.00$

$625.75 \quad 41.00$

1158.25

100.00

$0.00 \quad 0.00$ 
PAGE: 002

Harel, Drouin \& associés

Distribution cumulative du temps par employés

167 Dupuis Pierre

$$
\text { au } 31 / 12 / 1989
$$

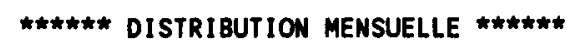

HEURES MOH-FACTURABLES

Code Description

005 Administration

010 Maladie

028 Vacances

025 Retard

040 Bureau

045 Transport

080 Cours conforrences

090 Cours conférences
090 Congès

740 Documentation

TOTAL HEURES NOW FACTURABLES

TOTAL DES HEURES $\begin{array}{lllll}\text { HEURES } & \boldsymbol{x} & \text { HEURES } \\ \text { REEL } & & \boldsymbol{x} & \text { MUOGET } \\ \text { REUR }\end{array}$

HEURES
REEL

HEURES
BUDGET

$x$

$\begin{array}{rrrrrrrr}15.75 & 19.03 & 0.00 & 0.00 & 54.75 & 6.10 & 0.00 & 0.00 \\ 5.00 & 0.60 & 0.00 & 0.00 & 11.00 & 1.20 & 0.00 & 0.00\end{array}$

$\begin{array}{rrrrrrrr}5.00 & 0.60 & 0.00 & 0.00 & 11.00 & 1.20 & 0.00 & 0.00 \\ 35.00 & 42.29 & 0.00 & 0.00 & 56.00 & 6.20 & 0.00 & 0.00\end{array}$

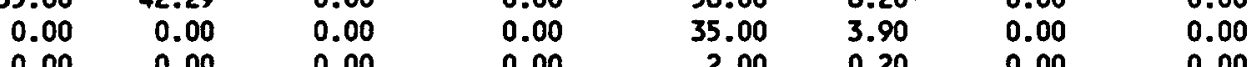

$\begin{array}{llllllll}0.00 & 0.00 & 0.00 & 0.00 & 2.00 & 0.20 & 0.00 & 0.00 \\ 0.00 & 0.00 & 0.00 & 0.00 & 8.25 & 0.90 & 0.00 & 0.00\end{array}$

$\begin{array}{llllllll}0.00 & 0.00 & 0.00 & 0.00 & 8.25 & 0.90 & 0.00 & 0.00 \\ 1.00 & 1.21 & 0.00 & 0.00 & 1.00 & 0.10 & 0.00 & 0.00\end{array}$

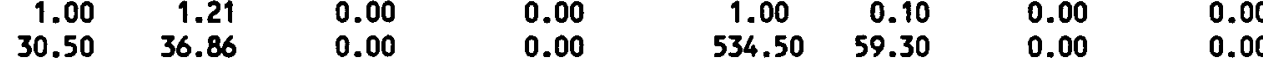

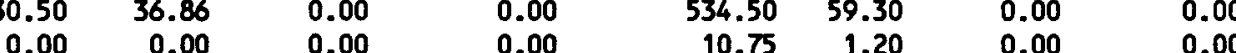

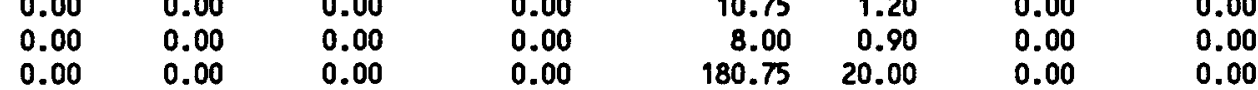

$\begin{array}{llllllll}82.75 & 52.90 & 0.00 & 0.00 & 902.00 & 59.00 & 0.00 & 0.00\end{array}$

$\begin{array}{lllllll}156.50 & 0.00 & 0.00 & 1527.75 & 0.00 & 0.00 & 0.00\end{array}$ 
PAGE: 003

167 Dupuis Pierre

HEURES SUPPLEMENTAIRES

Heures payées

MENSUELLE

2.50

SOLDE
Harel, Drouin \& associés

Distribution cumulative du temps par employés au 31/12/1989

CUMULATIF

111.50

0.00

111.50 


\section{Processus 2.7, Contrôler feuilles de temps}


Diagramme de flux de données 


\subsection{Vérifier le sommaire}

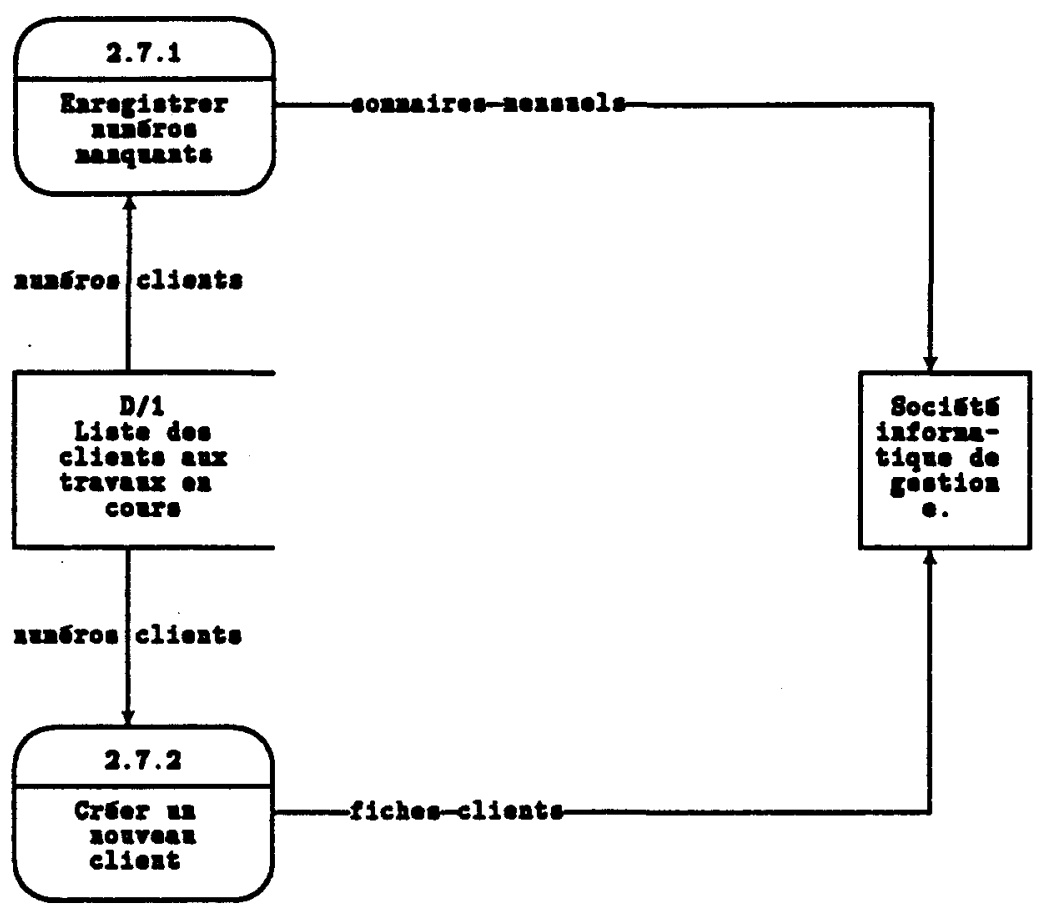


Processus de traitement 
Processus de traitement

\section{Déterminer feuilles de temps manquantes, 2.7.1}

\section{DESCRIPTION}

Ce sous-processus consiste à identifier automatiquement et régulièrement toutes les feuilles de temps qui n'ont pas été saisies par le système. Plusieurs fois par jour, le système exécute cette procédure et inscrit dans le fichier "D2/5 Feuilles de temps manquantes", les noms des personnes qui n'ont pas produites leurs feuilles de temps. Lorsqu'aucun nom n'est inscrit dans le fichier $\mathrm{D} 2 / 5$, le système indiquera que toutes les feuilles de temps de la semaine ont été reçues et ne permettra pas la saisie de nouvelles feuilles de temps.

ENTRÉE

Coordonnées, D2/3-2.7.1

Identification, D1-2.7.1

Transactions, D2/6-2.7.1

\section{SORTIE}

Feuilles complétées, 2.7.1-D2/3

Identification, 2.7.1-D2/5 


\section{RÉSUMÉ DE LA LOGIQUE}

Pour chaque semaine de feuille de temps non-complétée

Pour chaque enregistrement du fichier mantre employés-associés

si l'employé ou l'associé est actif

regarder, dans le fichier "D2/6 Transactions feuilles de temps", s'il y un enregistrement ayant le numéro lu dans le fichier maître employés-associés et la date d'une des journées comprises dans la feuille de temps

si oui,

vérifier dans le fichier des feuilles de temps manquantes s'il y a un enregistrement ayant le nom et le numéro lus dans le fichier maître et la date de la feuille de temps

si oui,

si non,

effacer l'enregistrement

vérifier dans le fichier des feuilles de temps manquantes, s'il y a un enregistrement ayant le nom et le numéro lus dans le fichier maître employés-associés et la date de la feuille de temps si non,

inscrire, dans le fichier des feuilles de temps manquantes, le numéro de l'associé ( qui est en fait le numéro de l'équipe), le numéro, le nom de la personne et la date de la feuille de temps.

Regarder, dans le fichier des feuilles de temps manquantes s'il y a des enregistrements ayant la date de la semaine

si oui,

sinon,

rien faire

inscrire dans le fichier des "D2/3 Coordonnées feuilles de temps" le code indiquant que toutes les feuilles de temps ont été reçues.

Calculer le temps supplémentaire (Processus 2.7.3) 
Processus de traitement

\section{Vérifier feuilles de temps manquantes, 2.7.2}

\section{DESCRIPTION}

Faisant partie intégrante du processus "2.7 Contrôler les feuilles de temps", ce processus vide à nous informer sur les personnes n'ayant pas produite leur feuille de temps.

\section{ENTRÉE}

date de la feuille de temps, a-2.7.2

numéro d'associé, a-2.7.2

identification, D2/5-2.7.2
SORTIE

feuilles de temps manquantes, 2.7.2-a

\section{RÉSUMÉ DE LA LOGIQUE}

demander le numéro de l'associé ( de l'équipe) pour lequel on désire imprimer la liste des feuilles de temps manquantes

demander la date de la feuille de temps pour laquelle on désire vérifier les feuilles de temps manquantes

demander si l'utilisateur désire un rapport imprimé ou consultation à l'écran si aucun numéro d'associé est fourni

imprimer ou afficher, selon le cas, tous les enregistrements du fichier D2/5 Feuilles de temps manquantes ayant la date de la feuille de temps fournie

si un numéro d'associé est fourni

imprimer ou afficher, selon le cas, tous les enregistrements du fichier D2/5 Feuilles de temps manquantes ayant le numéro de l'associé et la date de la feuille de temps fournie. 
Processus de traitement

\section{Calculer temps supplémentaire, 2.7.3}

\section{DESCRIPTION}

Processus automatique et transparent à l'utilisateur. Cette activité consiste à calculer, pour chaque journée de feuille de temps, le temps supplémentaire de chaque employé ou associé.

ENTRÉE

transactions, D2/6-2.7.3

coordonnées, D2/3-2.7.3

transactions, D10-2.7.3

\section{SORTIE}

temps supplémentaire, 2.7.3-D10

\section{RÉSUMÉ DE LA LOGIQUE}

Trier les transactions de feuilles de temps par jour et par numéro d'employé ou d'associé Pour chaque journée de feuille de temps inscrite dans le fichier des coordonnées de feuilles de temps

Pour chaque employé ou associés additionner ensemble le temps de toutes les transactions de feuilles de temps ayant le même numéro d'employé ou d'associé.

Soustraire du résultat obtenu, le nombre d'heures régulières de la journée (inscrit dans les coordonnées de feuilles de temps).

Créer, dans le fichier "D10 Cumulatif des transactions", un enregistrement ayant la date de la journée, le numéro de l'employé ou de l'associé, le code d'activité pour le temps supplémentaire et le résultat obtenu.

Éliminer, du fichier D2/6 Transactions feuilles de temps, toutes les transactions dont le code d'imputation signifie qu'elles ont été utilisées pour la mise à jour des travaux en cours. 
Flux de données 
Flux de données

\section{Date de la feuille de temps, a-2.7.2}

$\begin{array}{llll}\text { REF. SOURCE: } & a & \text { DESCRIPTION: } & \text { Employés autorisés } \\ \text { REF. DESTIN.: } & 2.7 .2 & \text { DESCRIPTION: } & \text { Vérifier feuilles de temps manquantes }\end{array}$

\section{DESCRIPTION DÉTAILLÉE}

La date de la feuille de temps pour laquelle nous désirons obtenir la liste des feuilles de temps manquantes. Cette date est validée avec les dates de feuilles de temps inscrites dans le fichier des coordonnées de feuilles de temps (D2/3). Si la date n'y est pas inscrite, un message est afficher à l'écran.

STRUCTURE DE DONNÉES INCLUSES

date: 8 caractères alphanumériques (ex.:03/11/89)

VOLUME

Harel Drouin et associés: 12

Harel Drouin Gestion Conseil: 3

Société d'informatique de gestion: 1

total par semaine: $\quad 16$

Hypothèse: Chaque associé peut demander la liste des feuilles de temps manquantes 
Flux de données

\section{Numéro d'associé, a-2.7.2}

REF. SOURCE: a DESCRIPTION: Employés autorisés

REF. DESTIN.: $\quad$ 2.7.2 DESCRIPTION: Vérifier feuilles de temps manquantes

DESCRIPTION DÉTAILLÉE

C'est le numéro de l'associé pour lequel nous désirons obtenir la liste des feuilles de temps manquantes.

STRUCTURE DE DONNÉES INCLUSES

numéro de l'associé: 3 caractères numériques

VOLUME

Harel Drouin et associés: 12

Harel Drouin Gestion Conseil: $\quad 3$

Société d'informatique de gestion: 1

total par semaine: $\quad 16$ 
Flux de données

\section{Feuilles de temps manquantes, 2.7.2-a}

$\begin{array}{llll}\text { REF. SOURCE: } & 2.7 .2 & \text { DESCRIPTION: } & \text { Vérifier feuilles de temps manquantes } \\ \text { REF. DESTIN.: } & a & \text { DESCRIPTION: } & \text { employés autorisés }\end{array}$

\section{DESCRIPTION DÉTAILLÉE}

Ce flux contient le numéro, le numéro de l'équipe (qui est en fait le numéro de l'associé), le nom des employés et associés, qui n'ont pas remis leur feuille de temps.

\section{STRUCTURE DE DONNÉES INCLUSES}

numéro: 3 caractères numériques

nom: 20 caractères alphanumériques

prénom: 20 caractères alphanumériques

no. de l'associé: 3 caractères numériques

\section{VOLUME}

Harel Drouin et associés:

$18^{(1)}$

Harel Drouin Gestion Conseil:

Société d'informatique de gestion: total par semaine: 
Flux de données

\section{Coordonnées, D2/3-2.7.1}

$\begin{array}{llll}\text { REF. SOURCE: } & \text { D2/3 } & \text { DESCRIPTION: } & \text { Coordonnées feuilles de temps } \\ \text { REF. DESTIN.: } & 2.7 .1 & \text { DESCRIPTION: } & \text { Déterminer feuilles de temps } \\ & & \text { manquantes }\end{array}$

\section{DESCRIPTION DÉTAILLÉE}

Contient les dates de feuilles de temps. Ces dates sont utilisées pour déterminer les feuilles de temps qui n'ont pas été reçues.

STRUCTURE DE DONNÉES INCLUSES

date: 8 caractères alphanumériques

1 ère journée: 8 caractères alphanumériques

nombre d'heures régulières pour la première journée: 1 caractère numérique

2 ème journée: 8 caractères alphanumériques

nombre d'heures régulières pour la deuxième journée: 1 caractère numérique 3 ème journée: 8 caractères alphanumériques nombre d'heures régulières pour la troisième journée: 1 caractère numérique 4 ème journée: 8 caractères alphanumériques nombre d'heures régulières pour la quatrième journée: 1 caractère numérique 5 ème journée: 8 caractères alphanumériques nombre d'heures régulières pour la cinquième journée: 1 caractère numérique 6 ème journée: 8 caractères alphanumériques nombre d'heures régulières pour la sixième journée: 1 caractère numérique 7 ème journée: 8 caractères alphanumériques nombre d'heures régulières pour la septième journée: 1 caractère numérique 


\section{VOLUME}

Harel Drouin et associés: $\quad 12$

Harel Drouin Gestion Conseil: 3

Société d'informatique de gestion: 1

total par semaine: $\quad 16$ 
Flux de données

\section{Feuilles complétées, 2.7.1-D2/3}

$\begin{array}{llll}\text { REF. SOURCE: } & 2.7 .1 & \text { DESCRIPTION: } & \begin{array}{l}\text { Déterminer feuilles de temps } \\ \text { manquantes }\end{array} \\ \text { REF. DESTIN.: } & \text { D2/3 } & \text { DESCRIPTION: } & \text { Coordonnées feuilles de temps }\end{array}$

\section{DESCRIPTION DÉTAILLÉE}

Ce flux contient seulement un caractère qui est inscrit dans le fichier "D2/3 Coordonnées feuilles de temps" indiquant que toutes les feuilles de temps ont été produites pour une semaine donnée. Ce caractère empêche d'effectuer la saisie de feuilles de temps pour une semaine pour laquelle toutes les feuilles de temps ont été saisies.

\section{STRUCTURE DE DONNÉES INCLUSES}

feuilles complétées: 1 caractère alphabétique ou numérique indiquant que toutes les feuilles de temps ont été reçues.

\section{VOLUME}

Harel Drouin et associés: $\quad 52^{(1)}$

Harel Drouin Gestion Conseil: $\quad 52$

Société d'informatique de gestion: $\underline{52}$

Total par semaine: $\quad 156$ 
Flux de données

\section{Identification, 2.7.1-D2/5}

REF. SOURCE: 2.7.1 DESCRIPTION: Déterminer feuilles manquantes

REF. DESTIN.: D2/5 DESCRIPTION: Feuilles de temps manquantes

\section{DESCRIPTION DÉTAILLÉE}

Contient le numéro de l'équipe (qui est, en fait, le numéro de l'associé), le numéro et le nom des employés-associés actifs qui n'ont pas produit leur feuille de temps.

\section{STRUCTURE DE DONNÉES INCLUSES}

numéro de la personne: 3 caractères numériques

nom de la personne: 20 caractères alphanumériques

prénom de la personne: 20 caractères alphanumériques

no. de l'associé: 3 caractères numériques

\section{VOLUME}

Harel Drouin et associés:

$18^{(1)}$

Harel Drouin Gestion Conseil:

3

Société d'informatique de gestion: $\underline{5}$

total par semaine:

26 
Flux de données

\section{Identification, D2/5-2.7.2}

REF. SOURCE: D2/5 DESCRIPTION: Feuilles de temps manquantes

REF. DESTIN.: $\quad$ 2.7.2 DESCRIPTION: Vérifier feuilles manquantes

\section{DESCRIPTION DÉTAILLÉE}

Contient le numéro de l'équipe (qui est, en fait, le numéro de l'associé), le numéro et le nom des employés-associés actifs qui n'ont pas produit leur feuille de temps et ce, pour une date et un associé donné.

STRUCTURE DE DONNÉES INCLUSES

numéro de la personne: 3 caractères numériques

nom de la personne: 20 caractères alphanumériques

prénom de la personne: 20 caractères alphanumériques

no. de l'associé: 3 caractères numériques

\section{VOLUME}

Harel Drouin et associés:

$18^{(1)}$

Harel Drouin Gestion Conseil:

3

Société d'informatique de gestion: $[\mathbf{5}$

Total par semaine: 
Flux de données

\title{
Identification, D1-2.7.1
}

REF. SOURCE: D1 DESCRIPTION: Fichier maître employés associés

REF. DESTIN.: 2.7.1 DESCRIPTION: Déterminer feuilles manquantes

\section{DESCRIPTION DÉTAILLÉE}

Contient le numéro de l'équipe (qui est, en fait, le numéro de l'associé), le numéro et le nom des employés-associés actifs. Ce flux est utilisé pour déterminer les employés et associés pour lesquels la feuille de temps n'a pas été saisie.

STRUCTURE DE DONNÉES INCLUSES

numéro de la personne: 3 caractères numériques

nom de la personne: 20 caractères alphanumériques

prénom de la personne: 20 caractères alphanumériques

no. de l'associé: 3 caractères numériques

\author{
VOLUME \\ Harel Drouin, et Associés $\quad: 180$ \\ Harel Drouin Gestion Conseil $\quad$ : 30 \\ Société d'informatique de gestion : $\underline{45}$ \\ Total par semaine $\quad: 255$
}


Flux de données

\section{Transactions, D2/6-2.7.1}

$\begin{array}{llll}\text { REF. SOURCE: } & \text { D2/6 } & \text { DESCRIPTION: } & \text { Transactions feuilles de temps } \\ \text { REF. DESTIN.: } & 2.7 .1 & \text { DESCRIPTION: } & \text { Déterminer feuilles manquantes }\end{array}$

\section{DESCRIPTION DÉTAILLÉE}

Ce flux contient toutes les transactions de feuilles de temps. Il est employé pour trouver, dans le fichier "D2/6 Transactions feuilles de temps", une transaction ayant le numéro de l'employé ou associé lu dans la fichier maître, la date d'une des journées de feuilles de temps comprises dans la semaine de feuilles de feuilles de temps. Si aucune transaction n'est trouvée, le numéro et le nom de l'employé ou associé sera inscrit dans le fichier "D2/5 Feuilles de temps manquantes".

\section{STRUCTURE DE DONNÉES INCLUSES}

numéro de la transaction: 5 caractères numériques

numéro de l'employé ou de l'associé: 3 caractères numériques

date de la transaction: 8 caractères alphanumériques

numéro du client: 8 caractères numériques

numéro de l'associé (1) $: 3$ caractères numériques

code de l'activité: 3 caractères numériques

temps: 4 caractères numériques ( 2 décimales)

code de validation: 1 caractère numérique, $1=$ transaction acceptée, $0=$ transaction refusée

code d'imputation: 1 caractère numérique, $1=$ transaction traitée, $0=$ transaction non traitée 
VOLUME

Harel Drouin, et Associés $\quad: 1,834,560$

Harel Drouin Gestion Conseil : $\quad 305,760$

Société d'informatique de gestion : 458,640

Total par semaine

$$
\text { : 2,617,305 }
$$


Flux de données

\section{Transactions, D2/6-2.7.3}

REF. SOURCE: D2/6 DESCRIPTION: Transactions feuilles de temps

REF. DESTIN.: $\quad 2.7 .3 \quad$ DESCRIPTION: Calculer temps supplémentaire

\section{DESCRIPTION DÉTAILLÉE}

Circulent, dans ce flux, toutes les transactions de feuilles de temps. Elles servent à calculer, pour chaque employé, pour chaque journée, le temps supplémentaire.

\section{STRUCTURE DE DONNÉES INCLUSES}

numéro de la transaction: 5 caractères numériques

numéro de l'employé ou de l'associé: 3 caractères numériques

date de la transaction: 8 caractères alphanumériques

numéro du client: 8 caractères numériques

numéro de l'associé ${ }^{(1)}: 3$ caractères numériques

code de l'activité: 3 caractères numériques

temps: 4 caractères numériques ( 2 décimales)

code de validation: 1 caractère numérique, $1=$ transaction acceptée, $0=$ transaction refusée

code d'imputation: 1 caractère numérique, $1=$ transaction traitée, $0=$ transaction non traitée

VOLUME

Harel Drouin, et Associés

: $1,834,560$

Harel Drouin Gestion Conseil

: 305,760

Société d'informatique de gestion : $\underline{458,640}$

Total par semaine

: 2,617,305 
Flux de données

\title{
Coordonnées, D2/3-2.7.3
}

\author{
REF. SOURCE: D2/3 DESCRIPTION: Coordonnées feuilles de temps \\ REF. DESTIN.: $\quad$ 2.7.3 DESCRIPTION: $\quad$ Calculer temps supplémentaire
}

\section{DESCRIPTION DÉTAILLÉE}

Contient les dates et le nombre d'heures régulières des journées comprises dans la feuille de temps qui vient d'être considérée par le système comme étant complète. Ce flux sert à calculer le temps supplémentaire pour chaque employé-associé/journée.

\section{STRUCTURE DE DONNÉES INCLUSES}

date de la feuille de temps: 8 caractères alphanumériques

1 ère journée: 8 caractères alphanumériques

nombre d'heures régulières pour la première journée: 1 caractère numérique

2 ème journée: 8 caractères alphanumériques

nombre d'heures régulières pour la deuxième journée: 1 caractère numérique

3 ème journée: 8 caractères alphanumériques

nombre d'heures régulières pour la troisième journée: 1 caractère numérique

4 ème journée: 8 caractères alphanumériques

nombre d'heures régulières pour la quatrième journée: 1 caractère numérique

5 ème journée: 8 caractères alphanumériques

nombre d'heures régulières pour la cinquième journée: 1 caractère numérique

6 ème journée: 8 caractères alphanumériques

nombre d'heures régulières pour la sixième journée: 1 caractère numérique

7 ème journée: 8 caractères alphanumériques

nombre d'heures régulières pour la septième journée: 1 caractère numérique 


\section{VOLUME}

Harel Drouin, et Associés $\quad: 52^{(1)}$

Harel Drouin Gestion Conseil : 52

Société d'informatique de gestion : 52

Total par semaine $\quad: 156$ 
Flux de données

\section{Temps supplémentaire, 2.7.3-D10}

REF. SOURCE: $\quad 2.7 .3 \quad$ DESCRIPTION: Calculer temps supplémentaire

REF. DESTIN.: D10 DESCRIPTION: Cumulatif des transactions

\section{DESCRIPTION DÉTAILLÉE}

Ce flux contient des transactions non facturables de feuilles de feuilles de temps dont le code d'activité signifie que c'est une transactions de temps supplémentaire et le temps est différent de zéro.

STRUCTURE DE DONNÉES INCLUSES

numéro de la transaction: 5 caractères numériques

numéro de l'employé ou de l'associé: 3 caractères numériques

date de la transaction: 8 caractères alphanumériques

numéro de l'associé: 3 caractères numériques

code de l'activité: 3 caractères numériques

temps: 4 caractères numériques ( 2 décimales)

VOLUME

Harel Drouin, et Associés $\quad$ : 65,700

Harel Drouin Gestion Conseil : 10,950

Société d'informatique de gestion : 16.425

Total par semaine $\quad: 93,075$ 


\section{Processus 5.2, Facturer les clients}


Diagramme de flux de données 
5.2 Enregistrer les recettes

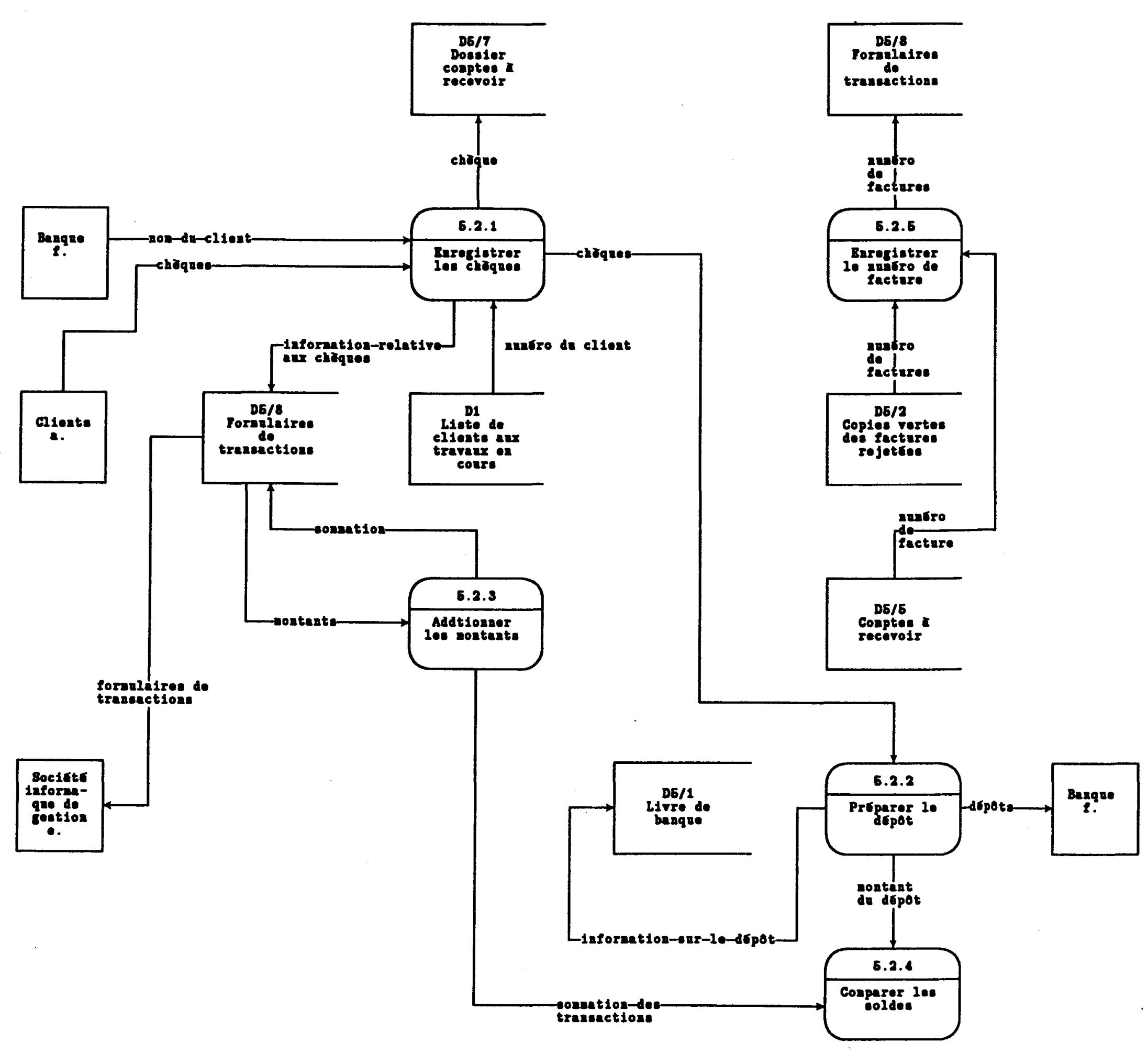




\section{'}




\section{Processus de traitement}


Processus de traitement

\section{Produire les factures, 5.2.1}

\section{DESCRIPTION}

Ce processus vise à élaborer le contenu d'une facture et ce à partir de l'auxiliaire des travaux en cours. Pour chaque client, l'associé ou le chef d'équipe consulte l'auxiliaire des travaux en cours afin de connaître les charges imputées (temps et dépenses) au dossier du client. A partir de cette information, il rédige ou dicte le contenu de la facture.

ENTRÉE

charges imputées, D7-5.2.1
SORTIE

facture, 5.2.1-5.2.2

RÉSUMÉ DE LA LOGIQUE

Regarder l'auxiliaire des travaux en cours pour connaître les charges imputées dans le compte du client

Dicter ou rédiger le contenu de la facture. 
Processus de traitement

\section{Dactylographier la facture, 5.2.2}

\section{DESCRIPTION}

Dactylographier, sur traitement de texte, le contenu de la facture dictée ou rédigée par le chef d'équipe ou l'associé.

Lorsque la facture est dactylographiée, l'original est envoyé au client et une copie verte est acheminée au département de la comptabilité. Cette copie sera employée pour effectuer la mise à jour de l'auxiliaire des travaux en cours et de l'auxiliaire des comptes à recevoir.

\section{ENTRÉE}

facture, 5.2.1-5.2.2
SORTIE

facture, 5.2.2-g

copie de la facture, 5.2.5-D5.2/1

RÉSUMÉ DE LA LOGIQUE

Dactylographier la facture à l'aide du traitement de texte.

Faire une copie de la facture sur du papier vert et envoyer cette copie au département de la comptabilité.

Envoyer la facture au client. 
Processus de traitement

\section{Saisir la facture, 5.2.3}

\section{DESCRIPTION}

La première étape est d'additionner tous les montants des factures à saisir. Le total ainsi obtenu sera comparé au total qui sera affiché sur l'écran lors de la saisie. A la saisie, un numéro de référence est attribuée à chaque facture. Ce numéro est assigné automatiquement par le système et, est inscrit sur le bas de chaque facture lors de son enregistrement. Par la suite, nous imprimons le contenu du fichier D5.2/2 des factures clients saisies, comparons le total inscrit au bas du rapport avec le total que nous avons calculé auparavant, et apportons les corrections nécessaires.

\section{ENTRÉE}

copies des factures, D5.2/1-5.2.3

nom du client, D2-5.2.3
SORTIE

copies des factures,5.2.3-D5.2/2

\section{RÉSUMÉ DE LA LOGIQUE}

Pour toutes les factures à saisir Additionner, sur une calculatrice à ruban, le total des factures à saisir et joindre le ruban au paquet de factures.

Pour toutes les factures à saisir

Enregistrer le numéro du client.

Vérifier si le nom du client inscrit sur l'écran est le même que celui sur la facture sinon corriger le numéro du client ou demander un correction du fichier client.

Inscrire la date de la facture.

Enregistrer le montant de la facture. Inscrire la description de la facture. Inscrire sur la facture, le numéro de référence assigné par le système. Changer de facture.

Comparer le total inșcrit sur l'écran avec le total des factures calculer préalablement. Faire imprimer le contenu du fichier "D5.2/2 Factures clients saisies".

Apporter les corrections nécessaires. 
Processus de traitement

\section{Enregistrer la vente, $\quad 5.2 .4$}

\section{DESCRIPTION DÉTAILLÉE}

Ce processus consiste à prendre toutes les factures qui ont été enregistrées dans le fichier D5.2/2 et à mettre à jour les différents auxiliaires et registres comptables. Chaque facture sera inscrite dans l'auxiliaire des travaux en cours et dans l'auxiliaire des comptes à recevoir. L'enregistrement de toutes les factures génère une écriture qui modifie, au grand-livre, le total des comptes à recevoir et le total des travaux en cours. Le numéro de l'écriture sera attribué par le système et l'écriture de journal sera la suivante:

Comptes à recevoir $\$ X X X X . X X$

Travaux en cours $\$ X X X X . X X$

et la description de l'écriture sera "Pour enregistrer les vente du mois ... ".

\section{ENTRÉE}

facture, D5.2/2-5.2.4

numéro d'écriture, D9-5.2.4
SORTIE

factures, 5.2.4-D7

factures, 5.2.4-D11

écritures de journal, 5.2.4-D8 


\section{RÉSUMÉ DE LA LOGIQUE}

Mettre le total de l'écriture égal à zéro

Ouvrir le fichier D5.2/2 qui contient les factures saisies. Pour toutes les factures inscrites dans le fichier:

Lire le numéro du client.

Lire le numéro de référence de la facture.

Lire la date de facture.

Lire le montant de la facture.

Lire, dans le fichier des numéros d'écritures, le numéro de-l'écriture pour le journal des ventes

Inscrire, dans l'auxiliaire des travaux en cours:

- le numéro de l'écriture

- le numéro du client

- le numéro de référence de la facture.

- la date de facture.

- le montant de la facture

- la description de la facture.

Inscrire, dans l'auxiliaire des comptes à recevoir:

- le numéro de l'écriture

- le numéro du client

- le numéro de référence de la facture.

- la date de facture.

- le montant de la facture

- la description de la facture.

Additionner le total de la facture au total de l'écriture.

Changer de facture.

Lorsqu'il n'y a plus de facture,

Inscrire, dans le fichier des écritures de journal, l'enregistrement qui contient les informations suivantes:

le numéro de compte des comptes à recevoir

le numéro de l'écriture

la date de la journée

le montant de l'écriture

la description de l'écriture ( généralement, honoraires du mois de ...)

Inscrire, dans le fichier des écritures de journal, l'enregistrement qui contient les informations suivantes:

le numéro de l'auxiliaire des travaux en cours

le numéro de l'écriture

la date de la journée

le montant de l'écriture

la description de l'écriture ( généralement, honoraires du mois de ...) 
Processus de traitement

\section{Saisir les chèques, 5.2 .5}

\section{DESCRIPTION}

Ce processus vise à enregistrer, dans le système informatique, les chèques envoyées par les clients. Cette information servira à la mise à jour de l'auxiliaire des comptes à recevoir. La saisie consiste donc à enregistrer le numéro du client, la date de son chèque, le numéro de référence de la facture, et le montant du chèque.

\section{ENTRÉE}

numéro, D2-5.2.5

numéro de facture, D11-5.2.5

chèques, $9-5.2 .5$
SORTIE

recettes, 5.2.5-D5.2/4

\section{RÉSUMÉ DE LA LOGIQUE}

Pour chaque chèque reçu Trouver le numéro du client Inscrire le numéro du client Trouver, dans l'auxiliaire des comptes à recevoir, le numéro de référence de la facture Inscrire le numéro de référence de la facture ${ }^{(1)}$ Inscrire la date du chèque Inscrire le montant du chèque 
Processus de traitement

\section{Enregistrer les recettes, 5.2.6}

\section{DESCRIPTION}

Ce processus visent à enregistrer, dans les registres comptables, l'information relative aux chèques envoyés par les clients. L'enregistrement de cette information aura pour conséquence de modifier les soldes des comptes à recevoir et de la banque.

ENTRÉE

recettes, D5.2/4-5.2.6

numéro d'écriture, D9-5.2.6
SORTIE

information sur paiement, 5.2.6-D11

écritures de journal, 5.2.6-D8

\section{RÉSUMÉ DE LA LOGIQUE}

Mettre le total des recettes égal à zéro.

Lire, dans le fichier "D9 Écritures de journal", le numéro de l'écriture correspondant au caisse recettes.

Pour toutes les recettes enregistrées dans le fichier "D5.2/4 Fichier temporaire des recettes" Lire un enregistrement

Inscrire, dans l'auxiliaire des comptes à recevoir, le numéro de l'écriture, le numéro du client, le numéro de référence de la facture, la date du chèque, le montant du chèque.

Additionner le montant de la recette au total des recettes.

Changer d'enregistrement

Inscrire, dans le fichier "D8 Écritures de journal", le numéro des comptes à recevoir, la date du système, le numéro de l'écriture, le montant de l'écriture et la description.

Inscrire, dans le fichier "D8 Écritures de journal", le numéro du compte de banque dans lequel les recettes sont déposées, la date du système, le numéro de l'écriture, le montant de l'écriture et la description. 
Flux de données 
Flux de données

\section{Charges imputées, D7-5.2.1}

REF. SOURCE: D7 DESCRIPTION: Auxiliaire des travaux en cours

REF. DESTIN.: $\quad$ 5.2.1 $\quad$ DESCRIPTION: Produire la facture

DESCRIPTION DÉTAILLÉE

Provient du rapport de l'auxiliaire des travaux en cours et contient le temps et les déboursés reliés au dossier de chaque client.

\section{STRUCTURE DE DONNÉES INCLUSES}

Les transactions sont présentées dans l'ordre chronologique.

Solde au début de la période:

temps travaillé: 9 caractères numériques dont deux décimales.

déboursés: 9 caractères numériques dont deux décimales

facturation: 9 caractères numériques dont deux décimales

profits et pertes: 9 caractères numériques dont deux décimales

Dans le cas du temps:

Pour chaque employé ou associé ayant travaillé sur le dossier:

numéro de l'employé ou associé: 3 caractères numériques

nom de l'employé: 40 caractères alphanumériques

code d'activité correspondant au temps: 3 caractères numériques

date de la transaction: 8 caractères alphanumériques

numéro de l'écriture de journal: 4 caractères alphanumériques

temps travaillé sur le dossier: 5 caractères numériques dont deux décimales.

taux horaire de l'employé ou associé: 3 caractères numériques

coût de la transaction: 9 caractères numériques dont deux décimales 
Dans le cas de déboursés facturables:

Pour chaque déboursé:

code d'activité correspondant au déboursé: 3 caractères numériques

date de la transaction: 8 caractères alphanumériques

numéro de l'écriture de journal: 4 caractères alphanumériques

description du déboursé: 40 caractères numériques

coût du déboursé: 9 caractères numériques dont deux décimales

\section{Dans le cas de facturation:}

Pour chaque facture:

code d'activité correspondant à une facture: 3 caractères numériques

date de la facture: 8 caractères alphanumériques

numéro de l'écriture de journal: 4 caractères alphanumériques

montant de la facture: 9 caractères numériques

description de la facture: 60 caractères alphanumériques

Dans le cas de transactions de profits et pertes:

code d'activité correspondant à une écriture de profits et pertes: 3 caractères numériques

date de la transaction: 8 caractères alphanumériques

numéro de l'écriture de journal: 4 caractères alphanumériques

montant de l'écriture: 9 caractères numériques

description de l'écriture: 60 caractères alphanumériques

Transactions de la période:

Total du temps travaillé: 9 caractères numériques

Total des déboursés: 9 caractères numériques

Total de la facturation: 9 caractères numériques

Total des profits et pertes: 9 caractères numériques 
Solde à la fin de la période:

temps travaillé: 9 caractères numériques dont deux décimales.

déboursés: 9 caractères numériques dont deux décimales facturation: 9 caractères numériques dont deux décimales profits et pertes: 9 caractères numériques dont deux décimales

Solde du compte du client: 13 caractères numériques

\section{VOLUME}

Harel Drouin, et associés: $\quad 12$ mois * 12 associés $=144$

Harel Drouin Gestion Conseil: 12 mois * 3 associés $=36$

Société d'informatique de gestion: 12 mois * 2 associés $=\underline{24}$

Total par mois: $\quad 204$ 
Flux de données

Nom, D2-5.2.1

REF. SOURCE: D2 DESCRIPTION: Fichier Maître clients

REF. DESTIN.: $\quad$ 5.2.1 DESCRIPTION: Produire les factures

DESCRIPTION DÉTAILLÉE

Contient le nom du client correspondant au numéro du client inscrit dans l'auxiliaire des travaux en cours. Ce nom est utilisé pour inscrire le nom du client dans le rapport nommé "Auxiliaire des travaux en cours".

STRUCTURE DE DONNÉES INCLUSES

nom du client: 60 caractères alphanumériques

VOLUME

Harel Drouin, et associés: $\quad 1500$

Harel Drouin Gestion Conseil: $\quad 300$

Société d'informatique de gestion: $\quad 100$

Total par semaine: $\quad 1900$ 
Flux de données

\section{Factures, 5.2.1-5.2.2}

REF. SOURCE: $\quad 5.2 .1 \quad$ DESCRIPTION: Produire la facture

REF. DESTIN.: $\quad 5.2 .2 \quad$ DESCRIPTION: Dactylographier la facture

\section{DESCRIPTION DÉTAILLÉE}

Contient les données statiques du client ainsi que le prix et la description du travail effectué dans son dossier. Contient également la description et le montant des déboursés encourus par la société dans l'exercice de son mandat.

\section{STRUCTURE DE DONNÉES INCLUSES}

numéro du client: 8 caractères numériques dont 5 pour le numéro du client et 3 pour le numéro du projet

nom du client: 60 caractères alphanumériques

adresse du client

numéro: 5 caractères alphanumériques

rue: 25 caractères alphanumériques

bureau: 5 caractères alphanumériques

ville: $\mathbf{3 0}$ caractères alphanumériques

province: 15 caractères alphanumériques

pays: 20 caractères alphanumériques

code postal: 7 caractères alphanumériques

description du travail effectué: environ 6 à 8 lignes de texte coût du travail effectué: 6 caractères numériques description des déboursés engagés: 60 caractères alphanumériques 
coût de chaque déboursé: 5 caractères numériques dont deux décimales total de la facture: 7 caractères numériques

\section{VOLUME}

Harel Drouin, et associés: environ 250

Harel Drouin Gestion Conseil: $\quad$ environ 20

Société d'informatique de gestion: environ 100

Total par mois: environ $\mathbf{3 7 0}$ 
Flux de données

Factures, 5.2.2-g

REF. SOURCE: $\quad 5.2 .2$ DESCRIPTION: Dactylographier la facture

REF. DESTIN.: $\quad$ g DESCRIPTION: Clients

DESCRIPTION DÉTAILLÉE

Contient les données statiques du client ainsi que le prix et la description du travail effectué dans son dossier. Contient également la description et le montant des déboursés encourus par la société dans l'exercice de son mandat.

\section{STRUCTURE DE DONNÉES INCLUSES}

numéro du client: 8 caractères numériques dont 5 pour le numéro du client et 3 pour le numéro du projet

nom du client: 60 caractères alphanumériques

adresse du client

numéro: 5 caractères alphanumériques

rue: 25 caractères alphanumériques

bureau: 5 caractères alphanumériques

ville: 30 caractères alphanumériques

province: 15 caractères alphanumériques

pays: 20 caractères alphanumériques

code postal: 7 caractères alphanumériques

description du travail effectué: environ 6 à 8 lignes de texte

coût du travail effectué: 6 caractères numériques

description des déboursés engagés: 60 caractères alphanumériques 
coût de chaque déboursé: 5 caractères numériques dont deux décimales

total de la facture: 7 caractères numériques

\section{VOLUME}

Harel Drouin, et associés: environ 250

Harel Drouin Gestion Conseil: $\quad$ environ 20

Société d'informatique de gestion: environ 100

Total par mois: $\quad$ environ 370 
Flux de données

\section{Copie de la facture, 5.2.2-D5.2/1}

$\begin{array}{llll}\text { REF. SOURCE: } & 5.2 .2 & \text { DESCRIPTION: } & \text { Dactylographier la facture } \\ \text { REF. DESTIN.: } & D 5.2 / 1 & \text { DESCRIPTION: } & \text { Copies des factures }\end{array}$

\section{DESCRIPTION DÉTAILLÉE}

La facture est généralement reproduite sur du papier de couleur et contient les données statiques du client ainsi que le prix et la description du travail effectué dans son dossier. Contient également la description et le montant des déboursés encourus par la société dans l'exercice de son mandat. 
STRUCTURE DE DONNÉES INCLUSES

numéro du client: 8 caractères numériques dont 5 pour le numéro du client et 3 pour le numéro du projet

nom du client: 60 caractères alphanumériques

adresse du client

numéro: 5 caractères alphanumériques

rue: 25 caractères alphanumériques

bureau: 5 caractères alphanumériques

ville: $\mathbf{3 0}$ caractères alphanumériques

province: 15 caractères alphanumériques

pays: 20 caractères alphanumériques

code postal: 7 caractères alphanumériques

description du travail effectué: environ 6 à 8 lignes de texte

coût du travail effectué: 6 caractères numériques

description des déboursés engagés: 60 caractères alphanumériques

coût de chaque déboursé: 5 caractères numériques dont deux décimales

total de la facture: 7 caractères numériques

VOLUME

Harel Drouin, et associés: environ 250

Harel Drouin Gestion Conseil: environ 20

Société d'informatique de gestion: environ 100

Total par mois: $\quad$ environ 370 
Flux de données

\section{Copie de la facture, D5.2/1-5.2.3}

REF. SOURCE: D5.2/1 DESCRIPTION: Copies des factures

REF. DESTIN.: $\quad 5.2 .3 \quad$ DESCRIPTION: Saisir les factures

\section{DESCRIPTION DÉTAILLÉE}

La copie de la facture est employée pour enregistrer les ventes dans le système informatique. Elle contient les données statiques du client ainsi que le prix et la description du travail effectué dans son dossier. Contient également la description et le montant des déboursés encourus par la société dans l'exercice de son mandat.

\section{STRUCTURE DE DONNÉES INCLUSES}

numéro du client: 8 caractères numériques dont 5 pour le numéro du client et 3 pour le numéro du projet

nom du client: 60 caractères alphanumériques

adresse du client

numéro: 5 caractères alphanumériques

rue: 25 caractères alphanumériques

bureau: 5 caractères alphanumériques

ville: 30 caractères alphanumériques

province: 15 caractères alphanumériques

pays: 20 caractères alphanumériques

code postal: 7 caractères alphanumériques

description du travail effectué: environ 6 à 8 lignes de texte

coût du travail effectué: 6 caractères numériques

description des déboursés engagés: 60 caractères alphanumériques 
coût de chaque déboursé: 5 caractères numériques dont deux décimales total de la facture: 7 caractères numériques

\section{VOLUME}

Harel Drouin, et associés: environ 250

Harel Drouin Gestion Conseil: $\quad$ environ 20

Société d'informatique de gestion: environ 100

Total par mois: $\quad$ environ $\mathbf{3 7 0}$ 
Flux de données

\title{
Copie de la facture, 5.2.3-D5.2/2
}

\author{
REF. SOURCE: $\quad 5.2 .3 \quad$ DESCRIPTION: Saisir les factures \\ REF. DESTIN.: $\quad$ D5.2/2 DESCRIPTION: Factures clients saisies
}

\section{DESCRIPTION DÉTAILLÉE}

La copie de la facture est employée pour enregistrer les ventes dans le système informatique et contient de l'information nécessaire à la mise à jour des différents auxiliaires.

\section{STRUCTURE DE DONNÉES INCLUSES}

code de transaction: 1 caractère numérique

numéro de l'écriture: 4 caractères numériques

date de la transaction: 8 caractères alphanumériques

numéro du client: 5 caractères numériques

numéro du projet: 3 caractères numériques

numéro de référence: 7 caractères numériques

description de la facture: 60 caractères alphanumériques

montant: 7 caractères numériques dont deux décimales

\section{VOLUME}

Harel Drouin, et associés: environ 250

Harel Drouin Gestion Conseil: environ 20

Société d'informatique de gestion: environ 100

Total par mois: environ $\mathbf{3 7 0}$ 
Flux de données

Factures, D5.2/2-5.2.4

REF. SOURCE: $\quad$ D5.2/2 DESCRIPTION: Factures clients saisies

REF. DESTIN.: $\quad 5.2 .4 \quad$ DESCRIPTION: Enregistrer la vente

\section{DESCRIPTION DÉTAILLÉE}

Contient toute l'information nécessaire à la mise à jour des auxiliaires des travaux en cours et des comptes à recevoir.

\section{STRUCTURE DE DONNÉES INCLUSES}

code d'activité: 3 caractères numériques (égal au code de transaction relié aux factures)

code de transaction: 1 caractère numérique

numéro de l'écriture: 4 caractères numériques

date de la transaction: 8 caractères alphanumériques

numéro du client: 5 caractères numériques

numéro du projet: 3 caractères numériques

numéro de référence: 7 caractères numériques

description de la facture: 60 caractères alphanumériques

montant: 7 caractères numériques dont deux décimales

VOLUME

Harel Drouin, et associés: environ 250

Harel Drouin Gestion Conseil: environ 20

Société d'informatique de gestion: environ 100

Total par mois: environ 370 
Flux de données

\section{Numéro d'écriture, D9-5.2.4}

REF. SOURCE: D9 DESCRIPTION: Numéro d'écritures

REF. DESTIN.: $\quad 5.2 .4 \quad$ DESCRIPTION: Saisir les factures

\section{DESCRIPTION DÉTAILLÉE}

Ce numéro est assigné par le système et sert à identifier un ensemble de transactions inscrites dans un journal et dans le grand-livre. Le numéro d'une écriture inscrit au grand-livre doit nous permettre de connaître l'origine de cette dernière. Par exemple, le numéro ah01 nous informe que nous faisons référence à une écriture qui provient du journal des achats (ah pour achat).

\section{STRUCTURE DE DONNÉES INCLUSES}

numéro de l'écriture: 4 caractères numériques

code de transaction: 1 caractère numérique ${ }^{(1)}$ 


\section{VOLUME}

Harel Drouin, et associés: 1

Harel Drouin Gestion Conseil: $\quad 1$

Société d'informatique de gestion: 1

Total par mois: 3 
Flux de données

\section{Factures, 5.2.4-D7}

$\begin{array}{llll}\text { REF. SOURCE: } & 5.2 .4 & \text { DESCRIPTION: } & \text { Enregistrer la vente } \\ \text { REF. DESTIN.: } & \text { D7 } & \text { DESCRIPTION: } & \text { Auxiliaire des travaux en cours }\end{array}$

DESCRIPTION DÉTAILLÉE

Contient toute l'information nécessaire à la mise à jour de l'auxiliaire des travaux en cours.

STRUCTURE DE DONNÉES INCLUSES

code d'activité: 3 caractères numériques (égal au code de d'activité relié aux factures)

code de transaction: 1 caractère numérique

numéro de l'écriture: 4 caractères numériques

date de la transaction: 8 caractères alphanumériques

numéro du client: 5 caractères numériques

numéro du projet: 3 caractères numériques

numéro de référence: 7 caractères numériques

description de la facture: 60 caractères alphanumériques

montant: 7 caractères numériques dont deux décimales 
VOLUME

Harel Drouin, et associés: environ 250

Harel Drouin Gestion Conseil: environ 20

Société d'informatique de gestion: environ 100

Total par mois: $\quad$ environ 370 
Flux de données

Factures, 5.2.4-D11

REF. SOURCE: $\quad$ 5.2.4 DESCRIPTION: Enregistrer la vente

REF. DESTIN.: D11 DESCRIPTION: Auxiliaire des comptes à recevoir

\title{
DESCRIPTION DÉTAILLÉE
}

Contient toute l'information nécessaire à la mise à jour de l'auxiliaire des comptes à recevoir.

\section{STRUCTURE DE DONNÉES INCLUSES}

code de transaction: 1 caractère numérique

numéro de l'écriture: 4 caractères numériques

date de la transaction: 8 caractères alphanumériques

numéro du client: 5 caractères numériques

numéro du projet: 3 caractères numériques

numéro de référence: 7 caractères numériques

description de la facture: 60 caractères alphanumériques

montant: 7 caractères numériques dont deux décimales

\author{
VOLUME \\ Harel Drouin, et associés: environ 250 \\ Harel Drouin Gestion Conseil: $\quad$ environ 20 \\ Société d'informatique de gestion: environ 100 \\ Total par mois: environ $\mathbf{3 7 0}$
}


Flux de données

\title{
Écritures de journal, 5.2.4-D8
}

\author{
REF. SOURCE: $\quad$ 5.2.4 DESCRIPTION: Enregistrer la vente \\ REF. DESTIN.: D8 DESCRIPTION: Écritures de journal
}

\section{DESCRIPTION DÉTAILLÉE}

Contient le numéro de l'écriture, la date de l'écriture, le numéro de compte affecté par l'écriture, le montant de l'écriture et la description. Le numéro de l'écriture contient des caractères permettant d'identifier le type de l'écriture. Ainsi une écriture qui concernent le journal des achats peut avoir le numéro suivant: ah01; ah pour achat.

\section{STRUCTURE DE DONNÉES INCLUSES}

numéro de l'écriture: 4 caractères alphanumériques

code de transaction: 1 caractère numérique ${ }^{(1)}$

date de l'écriture : 8 caractères alphanumériques

numéro de compte: 4 caractères numériques

numéro du sous-compte (succursale) s'il y a lieu: 2 caractères numériques

montant: maximum 8 caractères numériques dont 2 décimales

description: 60 caractères alphanumériques 
VOLUME

Harel Drouin, et associés: 1

Harel Drouin Gestion Conseil: 1

Société d'informatique de gestion: 1

Total par mois: 
Flux de données

Numéro, D2-5.2.5

REF. SOURCE: D2 DESCRIPTION: Fichier maître clients

REF. DESTIN.: $\quad 5.2 .5 \quad$ DESCRIPTION: Saisir les chèques

DESCRIPTION DÉTAILLÉE

C'est le numéro du client qui nous a fait parvenir un chèque.

STRUCTURE DE DONNÉES INCLUSES

numéro du client: 8 caractères numériques, 5 pour le numéro du client, 3 pour le numéro du projet

VOLUME

Harel Drouin, et associés: environ 250

Harel Drouin Gestion Conseil: environ 20

Société d'informatique de gestion: environ 100

Total par mois: environ $\mathbf{3 7 0}$ 
Flux de données

\title{
Chèques, g-5.2.5
}

\author{
REF. SOURCE: $\quad g \quad$ DESCRIPTION: Clients \\ REF. DESTIN.: $\quad 5.2 .5 \quad$ DESCRIPTION: Saisir les chèques
}

\section{DESCRIPTION DÉTAILLÉE}

Ce flux contient les chèques envoyés par les clients.

STRUCTURE DE DONNÉES INCLUSES

Date du chèque: 8 caractères alphanumériques

nom du destinataire: Harel Drouin \& associés

montant du chèque en chiffre: $\quad 7$ caractères numériques dont deux décimales

montant du chèque en lettres: environ 10 caractères alphanumériques

signature du client: environ 20 caractères alphanumériques

VOLUME

Harel Drouin, et associés: $\quad 300$

Harel Drouin Gestion Conseil: $\quad 20$

Société d'informatique de gestion: 20

Total par semaine: $\quad 340$ 
Flux de données

\title{
Numéro de facture, D11-5.2.5
}

\author{
REF. SOURCE: D11 DESCRIPTION: Auxiliaire des comptes à recevoir \\ REF. DESTIN.: $\quad 5.2 .5 \quad$ DESCRIPTION: Saisir les chèques
}

\section{DESCRIPTION DÉTAILLÉE}

Contient le numéro de facture inscrit dans l'auxiliaire des comptes à recevoir. Lorsqu'un client envoit un chèque, le personnel du département de la comptabilité regarde dans l'auxiliaire des comptes à recevoir afin d'identifier la facture correspondant au chèque reçu. Lors de la saisie du chèque, ce numéro sera inscrit dans la description de la transaction.

\section{STRUCTURE DE DONNÉES INCLUSES}

numéro: 7 caractères numériques

VOLUME

Harel Drouin, et associés: environ 250

Harel Drouin Gestion Conseil: environ 20

Société d'informatique de gestion: environ 100

Total par mois: environ 370 
Flux de données

\title{
Numéro d'écritures, D9-5.2.6
}

\author{
REF. SOURCE: D9 DESCRIPTION: Numéro d'écritures \\ REF. DESTIN.: $\quad 5.2 .6 \quad$ DESCRIPTION: Enregistrer les recettes
}

\section{DESCRIPTION DÉTAILLÉE}

Ce numéro est assigné par le système et sert à identifier un ensemble de transactions inscrites dans un journal et dans le grand-livre. Le numéro d’une écriture inscrit au grand-livre doit nous permettre de connaître l'origine de cette dernière. Par exemple, le numéro ah01 nous informe que nous faisons référence à une écriture qui provient du journal des achats (ah pour achat).

\section{STRUCTURE DE DONNÉES INCLUSES}

numéro de l'écriture: 4 caractères numériques

code de transaction: 1 caractère numérique ${ }^{(1)}$ 
VOLUME

Harel Drouin, et associés: 4

Harel Drouin Gestion Conseil: 4

Société d'informatique de gestion: $\underline{4}$

Total par mois: $\quad 12$ 
Flux de données

\section{Recettes, 5.2.5-D5.2/4}

REF. SOURCE.: $\quad 5.2 .5 \quad$ DESCRIPTION: Saisir les chèque

REF. DESTIN.: D5.2/4 DESCRIPTION: Fichier temporaire des recettes

\section{DESCRIPTION DÉTAILLÉE}

Contient toute l'information relative aux chèques saisis et sera utilisée pour mettre à jour l'auxiliaire des comptes à recevoir, les comptes à recevoir et l'encaisse.

\section{STRUCTURE DE DONNÉES INCLUSES}

code de transaction: 1 caractère numérique

numéro de l'écriture: 4 caractères numériques

numéro du client: 5 caractères numériques

numéro du projet: 3 caractères numériques

montant du chèque: 7 caractères numériques

numéro de référence de la facture: 7 caractères numériques

VOLUME

Harel Drouin, et associés: $\quad 300$

Harel Drouin Gestion Conseil: 20

Société d'informatique de gestion: 20

Total par semaine: $\quad 340$ 
Flux de données

\section{Recettes, D5.2/4-5.2.6}

REF. SOURCE: D5.2/4 DESCRIPTION: Fichier temporaire des recettes

REF. DESTIN.: $\quad 5.2 .6 \quad$ DESCRIPTION: Enregistrer les recettes

DESCRIPTION DÉTAILLÉE

Contient toute l'information relative aux chèques saisis et sera utilisée pour mettre à jour l'auxiliaire des comptes à recevoir, les comptes à recevoir et l'encaisse.

\section{STRUCTURE DE DONNÉES INCLUSES}

code de transaction: 1 caractère numérique

numéro de l'écriture: 4 caractères numériques

numéro du client: 5 caractères numériques

numéro du projet: 3 caractères numériques

montant du chèque: 7 caractères numériques

numéro de référence de la facture: 7 caractères numériques

\section{VOLUME}

Harel Drouin, et associés: $\quad 300$

Harel Drouin Gestion Conseil: $\quad 20$

Société d'informatique de gestion: 20

Total par semaine: $\quad 340$ 
Flux de données

\section{Information sur paiement, 5.2.6-D11}

REF. SOURCE: $\quad$ 5.2.6 DESCRIPTION: Enregistrer les recettes

REF. DESTIN.: D11 DESCRIPTION: Auxiliaire des comptes à recevoir

DESCRIPTION DÉTAILLÉE

Contient toute l'information relative aux chèques saisis et sera utilisée pour mettre à jour l'auxiliaire des comptes à recevoir.

STRUCTURE DE DONNÉES INCLUSES

code de transaction: 1 caractère numérique

numéro de l'écriture: 4 caractères numériques

numéro du client: 5 caractères numériques

numéro du projet: 3 caractères numériques

montant du chèque: 7 caractères numériques

numéro de référence de la facture: 7 caractères numériques

VOLUME

Harel Drouin, et associés: $23^{(1)}$

Harel Drouin Gestion Conseil: $\quad 5$

Société d'informatique de gestion: $\underline{5}$

Total par semaine: 
Flux de données

\section{Écritures de journal, 5.2.6-D8}

REF. SOURCE: 5.2.6 DESCRIPTION: Enregistrer les recettes

REF. DESTIN.: D8 DESCRIPTION: Écritures de journal

\section{DESCRIPTION DÉTAILLÉE}

Contient le numéro de l'écriture, la date de l'écriture, le numéro de compte affecté par l'écriture, le montant de l'écriture et la description. Le numéro de l'écriture contient des caractères permettant d'identifier le type de l'écriture. Ainsi une écriture qui concernent le journal des achats peut avoir le numéro suivant: ah01; ah pour achat.

\section{STRUCTURE DE DONNÉES INCLUSES}

numéro de l'écriture: 4 caractères alphanumériques

code de transaction: 1 caractère numérique ${ }^{(1)}$

date de l'écriture : 8 caractères alphanumériques

numéro de compte: 4 caractères numériques

numéro du sous-compte (succursale) s'il y a lieu: 2 caractères numériques

montant: maximum 8 caractères numériques dont 2 décimales

description: 60 caractères alphanumériques

1- code de transaction:

(1) transaction au journal général

(2) transaction au joumal des revenus

(3) transaction au joumal de la facturation

(4) transaction au caisse recettes

(5) transaction au caise déboursés

(6) transaction au joumal des achats 


\section{VOLUME}

$\begin{array}{ll}\text { Harel Drouin, et Associés } & : 23^{(1)} \\ \text { Harel Drouin Gestion Conseil } & : 5 \\ \text { Société d'informatique de gestion } & : \underline{5} \\ \text { Total par mois } & : 31\end{array}$
saisis à tous les jours, il y aura autant d'ecritures que de jours ourrables. 


\section{Processus 5.5, Payer les fournisseurs}




\section{Diagramme de flux de données}




\subsection{Payer les factures des fournisseurs}

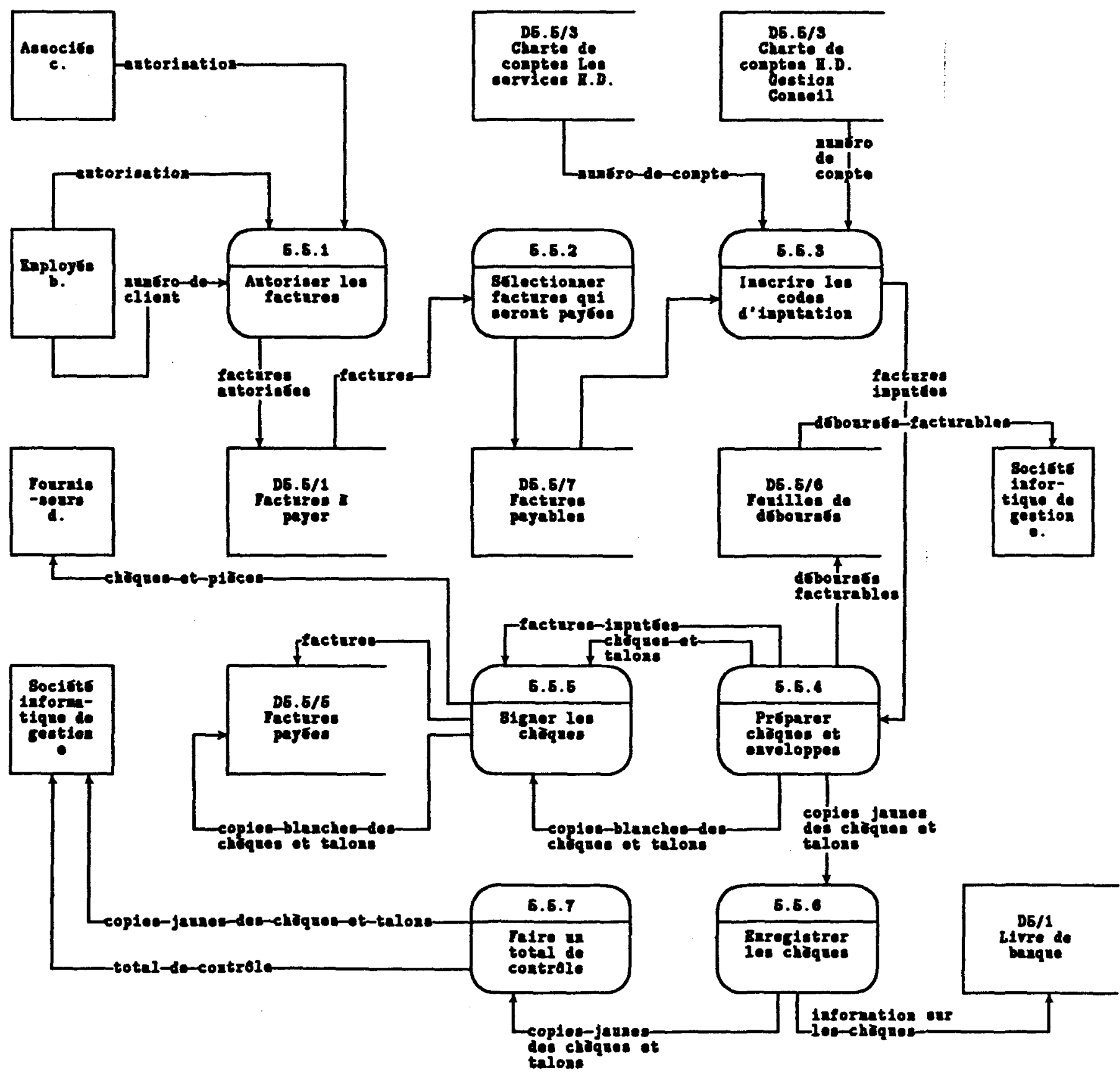




\section{Processus de traitement}


Processus de traitement

\section{Autoriser les factures, 5.5 .1}

\section{DESCRIPTION}

Ce processus consiste seulement à parapher les factures ou les comptes de dépenses par un associé ou un employé autorisé. Lorsqu'une facture est paraphée, cela signifie que le paiement en est autorisé.

ENTRÉE

Factures, d-5.5.1

Notes de crédits, d-5.5.1

Comptes de dépenses, b-5.5.1

autorisation, e-5.5.1

RÉSUMÉ DE LA LOGIQUE

Regarder le contenu de la facture

Parapher la facture
SORTIE

Factures autorisées,

5.5.1-D5.5/1 
Processus de traitement

\section{Inscrire les codes d'imputation, 5.5.2}

\section{DESCRIPTION}

Ce processus consiste, pour chaque facture ou compte de dépenses autorisé, à inscrire sur la facture ou le compte de dépenses, les numéros de comptes de grand-livre dans lesquels seront assignés les différents éléments de la facture et le numéro du compte fournisseurs.

\section{ENTRÉE}

Factures autorisées, D5.1/1-5.5.2

\# compte, D3-5.5.2

Numéro de compte, D5-5.5.2
SORTIE

Factures imputées, 5.5.2-D5.5/2

RÉSUMÉ DE LA LOGIQUE

Regarder dans la liste des fournisseurs le numéro de compte relié au fournisseur Inscrire sur la facture, le numéro de compte du fournisseur et le montant Inscrire les numéros des comptes de dépenses et les montants attribués à chacun 
Processus de traitement

\section{Saisir les factures, $\mathbf{5} .5 .3$}

\section{DESCRIPTION}

Consiste à enregistrer dans le système toute l'information inscrite sur chacune des factures lors du processus 5.5.2. Lors de la saisie des factures ou des comptes de dépenses, le système affichera le nom du fournisseur correspondant au numéro qui sera saisi ( les employés présentant des comptes de dépenses sont considérés comme fournisseur) et affichera la description du compte de grand-livre correspondant. Ce processus génèrera automatiquement les écritures de journal et reportera automatiquement les dites écritures dans le grand-livre.

\section{ENTRÉE}

Factures imputées, D5.5/2-5.5.3

Nom du fournisseur, D5-5.5.3

Description du compte, D3-5.5.3

Numéro client, D2-5.5.3

\section{SORTIE}

Factures, 5.5.3-D6

Dépenses, 5.5.3-D4

Écritures de journal, 5.5.3-D8 


\section{RÉSUMÉ DE LA LOGIQUE}

Mettre le cumulatif du montant à inscrire dans les comptes à payer à zéro

Lire, dans le fichier "D9 Numéro d'écriture", le numéro de l'écriture concernant le journal des achats

Pour toutes les factures à saisir

Enregistrer le numéro du fournisseur

Vérifier si le nom du fournisseur fourni par le système correspond au nom du fournisseur inscrit sur la facture

sinon vérifier le numéro du fournisseur inscrit sur la facture

Enregistrer la description de la facture (numéro de la facture fournisseur)

Enregistrer le montant de la facture

Additionner le montant de la facture au cumulatif à inscrire dans les comptes à payer

Pour tous les comptes du grand-livre inscrit sur la facture

Enregistrer le numéro de compte du grand-livre inscrit sur la facture

Vérifier la description du compte fournit par le système

Si la description du compte ne correspond pas au compte désiré, effectuer les corrections nécessaires

Enregistrer le numéro de la facture

Enregistrer le montant relié au numéro de compte saisi

Enregistrer:

- le numéro de l'écriture

- le code de transaction

- la date de la transaction

- le numéro de compte (pour la dépense)

- la description

Enregistrer:

le montant dans le fichier "D8 Écritures de journal"

- le numéro de l'écriture

- le code de transaction

- la date de la transaction

- le numéro des comptes à payer

- la description

- le montant des comptes à payer dans le fichier "D8 Écritures de journal" 
Processus de traitement

\section{Imprimer liste des factures à payer, 5.5.4}

DESCRIPTION

Cette opération consiste à imprimer toutes les factures arrivée à échéance. Pour ce faire, le système calculera, pour chaque facture inscrite dans l'auxiliaire des comptes à payer, la date d'échéance de la facture.

\section{ENTRÉE}

date dûe, D5-5.5.4

factures, D6-5.5.4

date, $\mathbf{f - 5 . 5 . 4}$
SORTIE

liste des factures à payer, 5.5.4-D5.5/4

date,5.5.4-D5.5/5

nom du fournisseur, D5-5.5.5

\section{RÉSUMÉ DE LA LOGIQUE}

Demander à l'employé du personnel administratif la date pour laquelle il désire imprimer la liste des factures dûes.

Valider la date fournie par l'usager (elle doit être plus grande ou égale à celle du système). Pour chaque facture inscrite dans l'auxiliaire des comptes à payer

Regarder dans "D5 Fichier maître fournisseurs" le nombre de jours après lequel les factures doivent être payées

Pour chaque facture de chaque fournisseur

Calculer la date d'échéance de la facture

Si la date d'échéance est inférieure ou égale à la date fournie

imprimer le numéro du fournisseur, le nom du fournisseur, la facture, son montant dans la liste des factures à payer

Changer de facture 
Processus de traitement

\section{Retenir certaines factures, $\mathbf{5} .5 .5$}

\section{DESCRIPTION}

Consiste à choisir, parmi les factures imprimées sur la liste des factures à payer, celles qui seront retenues ou celles pour lesquelles un paiement partiel sera effectué.

\section{ENTRÉE}

Liste des factures à payer, 5.5.4-5.5.5

date,D5.5/5-5.5.5

\section{SORTIE}

paiements partiels ou retenus, 5.5.5-D6

factures payées, $5.5 .5-D 5.5 / 3$

information sur paiement,5.5.5-D5.5/6

\section{RÉSUMÉ DE LA LOGIQUE}

Demander le numéro du fournisseur

Regarder dans "D5 Fichier maître fournisseurs" le nombre de jours après lequel les factures doivent être payées

Pour chaque facture du fournisseur

Calculer la date d'échéance de la facture

Si la date d'échéance est inférieure ou égale à la date de sélection (enregistrée dans le fichier D5.5/5)

afficher la facture, son montant

demander si on désire retenir cette facture ou effectuer un paiement partiel si retenir facture

inscrire le code indiquant que la facture sera retenue

si paiement partiel

inscrire le code indiquant que la facture sera payée partiellement demander le montant du paiement

inscrire le numéro du fournisseur, la date de la facture, le numéro de la

Changer de facture facture, le montant du paiement dans le fichier des paiements partiels

Retirer du lot de facture à payer, toutes les factures qui seront payées ${ }^{(1)}$ 
Processus de traitement

\section{Imprimer les chèques, 5.5 .6}

\section{DESCRIPTION}

Consiste à imprimer un chèque par fournisseur pour lequel des factures sont payées. Le talon du chèque contient la liste des factures couvertes par le chèques émis. La copie du chèque est rangée avec les factures payées.

\section{ENTRÉE}

factures payables, D6-5.5.6

montant, D5.5/6-5.5.6

coordonnées,D5-5.5.6

\section{SORTIE}

copies des chèques, 5.5.6-

D5.5/3

écritures de journal, 5.5.6-D8

chèques, 5.5.6-d

chèques, 5.5.6-b

information sur paiement, 5.5.6-D6 


\section{RÉSUMÉ DE LA LOGIQUE}

Pour chaque fournisseur

Mettre le montant du chèque à zéro(1)

Pour toutes les factures qui ont le code indiquant qu'elles seront payées ou celles qui ont un code indiquant qu'il y aura un paiement partiel

Inscrire le numéro de la facture, la date de la facture sur le talon du chèque

S'il y a un code indiquant un paiement partiel

lire, dans le fichier "D5.5/6" des paiements partiels, le montant du paiement inscrire le montant du paiement sur le talon du chèque

sinon,

Inscrire le montant de la facture sur le talon du chèque

Dans l'auxiliaire des comptes à payer, inscrire:

- le numéro de l'écriture

- le code de transaction

- le numéro du fournisseur,

- le numéro de la facture

- le montant payé

- le numéro du chèque

- la description

Additionner le montant payé au montant du chèque

Imprimer le nom et l'adresse du fournisseur, le montant du chèque sur le chèque

Dans le fichier "D8 Écritures de journal", inscrire:

- le numéro de l'écriture

- le code de transaction

- la date de la transaction

- le numéro des comptes à payer

- le numéro du chèque

- le montant du chèque

Dans le fichier "D8 Écritures de journal", inscrire:

- le numéro de l'écriture

- le code de transaction

- la date de la transaction

- le numéro du compte de banque

- le numéro du chèque

- le montant du chèque 
Processus de traitement

\section{Imputer les dépenses, $\mathbf{5 . 5 . 7}$}

\section{DESCRIPTION}

Ce processus visent à reporter toutes les dépenses encourues par les services Harel Drouin dans les registres comptables de Harel Drouin et associés. Est également inclu dans ce processus, l'affectation, dans le fichier "D10 Cumulatif des transactions", des dépenses non facturables provenant des comptes de dépenses des employés.

ENTRÉE

dépenses,D4-5.5.7

\section{SORTIE}

écriture de journal,5.5.7-D8

dépenses facturables, 5.5.7-D7

dépenses non-facturables, 5.5.7-D10 


\section{RÉSUMÉ DE LA LOGIQUE}

Mettre le total à imputer aux travaux en cours égal à zéro.

Pour toutes les dépenses enregistrées dans le fichier

Si le numéro de compte signifie que la dépense est facturable:

si oui:

Inscrire, dans l'auxiliaire des travaux en cours:

- le code de transaction

- le numéro de l'écriture

- le numéro du client

- le montant à imputé

- la date de la transaction

- la description de la dépenso

additionner le montant de la dépense au montant à imputer aux travaux en cours. additionner le montant de la dépense au montant à imputer dans le compte Intercompagnie

sinon:

inscrire, dans le fichier des écritures de journal de la société:

- le code de transaction

- le numéro de l'écriture

- le numéro du compte

- le montant à imputé

- la date de la transaction

- la description de la dépense

inscrire, dans le fichier des écritures de journal de la corporation:

- le code de transaction

- le numéro de l'écriture

- le numéro du compte

- le montant à imputé (négatif pour crédit)

- la date de la transaction

- la description de la dépense

additionner le montant de la dépense au montant à imputer dans le compte Intercompagnie

inscrire, dans le fichier "D10 Cumulatif des transactions"

numéro de l'employé ou de l'associé: 3 caractères numériques

date de la transaction: 8 caractères alphanumériques

code de l'activité: 3 caractères alphanumériques

description: 40 caractères alphanumériques

montant: 5 caractères numériques

Inscrire, dans le fichier des écritures de journal de la société:

- le code de transaction

- le numéro de l'écriture

- le numéro du compte des travaux en cours

- le montant à imputé

- la date de la transaction

- la description de la transaction (1) 
- le code de transaction

- le numéro de l'écriture

- le numéro du compte Inter compagnie

- le montant à imputé (le montant des dépenses plus le montant imputé aux travaux en cours)

- la date de la transaction (1)

- la description de la transaction

Inscrire, dans le fichier des écritures de journal de la corporation:

- le code de transaction

- le numéro de l'écriture

- le numéro du compte Inter compagnie

- le montant à imputé

- la date de la transaction

- la description de la transaction (2)

Les écritures seront donc les suivantes:

dans le cas de la Société Harel Drouin:

TravauX en cours $\quad X X X$

Dépenses $\quad X X X$

Inter-compagnie $\quad X X X$

dans le cas de la corporation "Les Services Harel Drouin":

Inter-compagnie $\quad X X X$

Dépenses $\quad X X X$ 
Processus de traitement

\section{Enregistrer les nouveaux fournisseurs, 5.5.8}

\section{DESCRIPTION}

Ce processus consiste à enregistrer le nom, l'adresse et les modalité de paiement de chaque fournisseur.

ENTRÉE

RÉSUMÉ DE LA LOGIQUE

Enregistrer le nom du fournisseur

Enregistrer l'adresse du fournisseur

Enregistrer les modalité de paiement

\section{SORTIE}

information fournisseur, 5.5.8-D5 
Flux de données 
Flux de données

\title{
Factures, d-5.5.1
}

\author{
REF. SOURCE: d \\ DESCRIPTION: Fournisseurs \\ REF.DESTIN.: 5.5.1 \\ DESCRIPTION: Autoriser les factures
}

\section{DESCRIPTION DÉTAILLÉE}

Contient toutes les factures reliées à des achats, des locations ou des acquisitions et envoyées par les fournisseurs.

\section{STRUCTURE DE DONNÉES INCLUSES}

nom du fournisseur: 60 caractères alphanumériques

adresse du fournisseur: environ 150 caractères alphanumériques

numéro de la facture: maximum 10 caractères alphanumériques

date de la facture: 8 caractères alphanumériques

montant de la facture: maximum 8 caractères numériques dont 2 décimales

description des articles commandés: environ 60 caractères alphanumériques

coût des articles commandés: maximum 7 caractères numériques dont deux décimales

adresse de la facturation: environ 150 caractères alphanumériques

adresse de la livraison: environ 150 caractères alphanumériques

échéance: 25 caractères alphanumériques (généralement 30 jours) 
VOLUME

Harel Drouin, et Associés : 350

Harel Drouin Gestion Conseil : 25

Société d'informatique de gestion : 25

Total par mois $\quad: \mathbf{4 0 0}$ 
Flux de données

\section{Notes de crédit, d-5.5.1}

REF. SOURCE: $d$

DESCRIPTION: Fournisseurs

REF. DESTIN.: $\quad 5.5 .1$

DESCRIPTION: Autoriser les factures

DESCRIPTION DÉTAILLÉE

Provient des fournisseurs. C'est une note mentionnant qu'un crédit relatif à une facture nous est accordé. La note de crédit contient pratiquement la même information qu'une facture, cependant, il n'y a pas de description d'article. La note de crédit contient le numéro de facture auquel elle fait référence.

STRUCTURE DE DONNÉES INCLUSES

date le la note de crédit: 8 caractères alphanumériques

nom du fournisseur: 60 caractères alphanumériques

adresse du fournisseur: environ 150 caractères alphanumériques

numéro de la facture reliée à la note: maximum 10 caractères alphanumériques

montant de la note de crédit: maximum 8 caractères numériques dont 2 décimales

VOLUME

Harel Drouin, et Associés $\quad: 5$

Harel Drouin Gestion Conseil : : 5

Société d'informatique de gestion : $\underline{5}$

Total par mois $\quad: 15$ 
Flux de données

\section{Autorisation, e-5.5.1}

REF. SOURCE: $P$

DESCRIPTION:

Associés employés autorisés

REF. DESTIN.: $\quad 5.5 .1$

DESCRIPTION: Autoriser les factures

\section{DESCRIPTION DÉTAILLÉE}

L'employé ou l'associé qui autorise une facture ou un compte de dépenses appose ses initiales ou sa signature sur la facture ou le compte de dépenses.

\section{STRUCTURE DE DONNÉES INCLUSES}

nom de l'employé ou associé qui autorise: 30 caractères alphanumériques

initiales: maximum 3 caractères alphanumériques

\section{VOLUME}

Harel Drouin, et Associés

$: 350^{(1)}+100^{(2)}$

Harel Drouin Gestion Conseil

$: 25+20$

Société d'informatique de gestion

$: 25+25$

Total par mois

$: 400+145=545$

1- pour les factures 
Flux de données

\section{Comptes de dépenses, b-5.5.1}

REF. SOURCE: $b$

REF. DESTIN.: $\quad 5.5 .1$
DESCRIPTION: Membre du personnel

DESCRIPTION: Autoriser les factures

\section{DESCRIPTION DÉTAILLÉE}

Un compte de dépenses contient un ensemble de dépenses facturables encourues par un employé ou un associé. II contient également des dépenses non-facturables.

\section{STRUCTURE DE DONNÉES INCLUSES}

date: 8 caractères alphanumériques

numéro de l'employé: 3 caractères numériques

nom de l'employé: 40 caractères alphanumériques

20 pour le nom, 20 pour le prénom

approbation: nom ou initiale d'un associé

section des dépenses facturables:

code de dépense: 3 caractères numériques

numéro du client: 8 caractères numériques

5 caractères numériques pour le numéro du client, 3 caractères numériques

pour le numéro du mandat

nom du client:70 caractères alphanumériques

description de la dépense: 60 caractères alphanumériques

montant: 7 caractères numériques dont deux décimales 
section des dépenses non-facturables:

code de dépense: 3 caractères numériques

description de la dépense: 60 caractères alphanumériques

$\begin{array}{ll}\text { VOLUME } & \\ \text { Harel Drouin, et associés } & : 00 \\ \text { Harel Drouin Gestion Conseil } & : 20 \\ \text { Société d'informatique de gestion } & : \underline{25} \\ \text { Total par mois } & : 145\end{array}$


Flux de données

\section{Factures autorisées, 5.5.1-D5.5/1}

REF. SOURCE: 5.5 .1

REF. DESTIN.: D5.5/1
DESCRIPTION: Autoriser les factures

DESCRIPTION: Factures autorisées

\section{DESCRIPTION DÉTAILLÉE}

Contient des factures ou des comptes de dépenses ayant la signature ou l'initiale de l'employé autorisé ou de l'associé.

\section{STRUCTURE DE DONNÉES INCLUSES}

\section{Dans le cas de factures:}

nom du fournisseur: 60 caractères alphanumériques

adresse du fournisseur: environ 150 caractères alphanumériques

numéro de la facture: maximum 10 caractères alphanumériques

date de la facture: 8 caractères alphanumériques

montant de la facture: maximum 8 caractères numériques dont 2 décimales

description des articles commandés: environ 60 caractères alphanumériques

coût des articles commandés: maximum 7 caractères numériques dont deux décimales

adresse de la facturation: environ 150 caractères alphanumériques

adresse de la livraison: environ 150 caractères alphanumériques

échéance: 25 caractères alphanumériques (généralement 30 jours)

nom de l'employé ou associé qui autorise: 30 caractères alphanumériques

initiales: maximum 3 caractères alphanumériques 
Dans le cas de comptes de dépenses:

date: 8 caractères alphanumériques

numéro de l'employé: 3 caractères numériques

nom de l'employé: 40 caractères alphanumériques

20 pour le nom, 20 pour le prénom

approbation: nom ou initiale d'un associé

section des dépenses facturables:

code de dépense: 3 caractères numériques

numéro du client: 8 caractères numériques

5 caractères numériques pour le numéro du client, 3 caractères numériques

pour le numéro du mandat

nom du client:70 caractères alphanumériques

description de la dépense: 60 caractères alphanumériques

montant: 7 caractères numériques dont deux décimales

section des dépenses non-facturables:

code de dépense: 3 caractères numériques

description de la dépense: 60 caractères alphanumériques 


\section{VOLUME}

$\begin{array}{ll}\text { Harel Drouin, et Associés } & : 350^{(1)}+100^{(2)} \\ \text { Harel Drouin Gestion Conseil } & : 25+20 \\ \text { Société d'informatique de gestion } & : 25+25 \\ \text { Total par mois } & : 400+145=545\end{array}$

1- pour les factures

2- pour les comptes de dépenses 
Flux de données

\title{
Factures autorisées, D5.5/1-5.5.2
}

\author{
REF. SOURCE: D5.5/1 DESCRIPTION: Factures autorisées \\ REF. DESTIN.: $\quad 5.5 .2 \quad$ DESCRIPTION: Inscrire les codes d'imputation
}

\section{DESCRIPTION DÉTAILLÉE}

Contient des factures ou comptes de dépenses ayant la signature ou l'initiale de l'employé autorisé ou de l'associé.

\section{STRUCTURE DE DONNÉES INCLUSES}

\section{Dans le cas de facture:}

nom du fournisseur: 60 caractères alphanumériques

adresse du fournisseur: environ 150 caractères alphanumériques

numéro de la facture: maximum 10 caractères alphanumériques

date de la facture: 8 caractères alphanumériques

montant de la facture: $\quad$ maximum 8 caractères numériques dont 2 décimales

description des articles commandés: environ 60 caractères alphanumériques

coût des articles commandés: maximum 7 caractères numériques dont deux décimales

adresse de la facturation: environ 150 caractères alphanumériques

adresse de la livraison: environ 150 caractères alphanumériques

échéance: 25 caractères alphanumériques (généralement 30 jours)

nom de l'employé ou associé qui autorise: 30 caractères alphanumériques

initiales: maximum 3 caractères alphanumériques 


\section{Dans le cas de comptes de dépenses:}

date: 8 caractères alphanumériques

numéro de l'employé: 3 caractères numériques

nom de l'employé: 40 caractères alphanumériques

20 pour le nom, 20 pour le prénom

approbation: nom ou initiale d'un associé

section des dépenses facturables:

code de dépense: 3 caractères numériques

numéro du client: 8 caractères numériques

5 caractères numériques pour le numéro du client, 3 caractères numériques

pour le numéro du mandat

nom du client:70 caractères alphanumériques

description de la dépense: 60 caractères alphanumériques

montant: 7 caractères numériques dont deux décimales

section des dépenses non-facturables:

code de dépense: 3 caractères numériques

description de la dépense: 60 caractères alphanumériques

montant: 7 caractères numériques dont deux décimales

\section{VOLUME}

Harel Drouin, et Associés $\quad: 350^{(1)}+100^{(2)}$

Harel Drouin Gestion Conseil $\quad: \quad 25+20$

Société d'informatique de gestion : $25+25$

Total par mois $\quad: 400+145=545$

1- pour les factures

2- pour les comptes de dépenses 
Flux de données

\section{Numéro de compte, D3-5.5.2}

REF. SOURCE: D3

REF. DESTIN.: $\quad 5.5 .2$
DESCRIPTION: Grand-livre

DESCRIPTION: Inscrire les codes d'imputation

\section{DESCRIPTION DÉTAILLÉE}

Contient le numéro du compte et du sous-compte de grand-livre dans lequel sera imputer la dépense ou l'acquisition.

STRUCTURE DE DONNÉES INCLUSES

numéro de compte: 4 caractères numériques

numéro du sous-compte (succursale) s'il y a lieu: 2 caractères numériques

VOLUME

Harel Drouin, et Associés

$: 350^{(1)}+100^{(2)}$

Harel Drouin Gestion Conseil

: $25+20$

Société d'informatique de gestion

$: 25+25$

Total par mois

$: 400+145=545$

1- pour les factures

2- pour les comptes de dépenses 
Flux de données

\# compte, D5-5.5.2

REF. SOURCE: D5

REF. DESTIN.: $\quad \mathbf{5 . 5 . 2}$
DESCRIPTION: Fichier maître fournisseur

DESCRIPTION: Inscrire les codes d'imputation

\section{DESCRIPTION DÉTAILLÉE}

Contient un numéro de compte de l'auxiliaire des comptes à payer qui est, en fait un sous-compte des comptes à payer. Ce numéro représente le numéro du fournisseur, dans le cas d'un paiement de facture, ou le numéro d'un employé, dans le cas du remboursement d'un compte de dépenses.

\section{STRUCTURE DE DONNÉES INCLUSES}

numéro du sous-compte ou du fournisseur: 4 caractères numériques

\section{VOLUME}

Harel Drouin, et Associés

$: 350^{(1)}+100^{(2)}$

Harel Drouin Gestion Conseil

$: 25+20$

Société d'informatique de gestion

$: 25+25$

Total par mois

$: 400+145=545$

1- pour les factures

2- pour les comptes de dépenses 
Flux de données

\title{
Factures imputées, 5.5.2-D5.5/2
}

\author{
REF. SOURCE: 5.5 .2 DESCRIPTION: Inscrire les codes d'imputation \\ REF. DESTIN.: D5.5/2 DESCRIPTION: Factures imputées
}

\section{DESCRIPTION DÉTAILÉE}

Factures ou comptes de dépenses ayant au minimum 2 numéros de comptes de grandlivre et des montants associés à chaque compte.

\section{STRUCTURE DE DONNÉES INCLUSES}

\section{Dans le cas de facture:}

nom du fournisseur: 60 caractères alphanumériques

adresse du fournisseur: environ 150 caractères alphanumériques

numéro de la facture: maximum 10 caractères alphanumériques

date de la facture: 8 caractères alphanumériques

montant de la facture: $\quad$ maximum 8 caractères numériques dont 2 décimales

description des articles commandés: environ 60 caractères alphanumériques

coût des articles commandés: maximum 7 caractères numériques dont deux décimales

adresse de la facturation: environ 150 caractères alphanumériques

adresse de la livraison: environ 150 caractères alphanumériques

échéance: 25 caractères alphanumériques (généralement 30 jours)

nom de l'employé ou associé qui autorise: 30 caractères alphanumériques

initiales: maximum 3 caractères alphanumériques

numéro de compte du grand-livre ${ }^{(1)}: 4$ caractères numériques

numéro du sous-compte du grand-livre ( $s^{\prime i l}$ y a lieu) : 2 caractères numériques 
montant: 6 caractères numériques dont deux décimales

numéro de compte du fournisseur: 4 caractères numériques

montant: 6 caractères numériques

Dans le cas de comptes de dépenses:

date: 8 caractères alphanumériques

numéro de l'employé: 3 caractères numériques

nom de l'employé: 40 caractères alphanumériques

20 pour le nom, 20 pour le prénom

approbation: nom ou initiale d'un associé

section des dépenses facturables:

code de dépense: 3 caractères numériques

numéro du client: 8 caractères numériques

5 caractères numériques pour le numéro du client, 3 caractères numériques pour le numéro du mandat

nom du client:70 caractères alphanumériques

description de la dépense: 60 caractères alphanumériques

montant: 7 caractères numériques dont deux décimales

section des dépenses non-facturables:

code de dépense: 3 caractères numériques

description de la dépense: 60 caractères alphanumériques

montant: 7 caractères numériques dont deux décimales 
VOLUME

Harel Drouin, et Associés $\quad: 350^{(1)}+100^{(2)}$

Harel Drouin Gestion Conseil $\quad: 25+20$

Société d'informatique de gestion : $25+25$

Total par mois $\quad: 400+145=545$

1- pour les factures

2- pour les comptes de dépenses 
Flux de données

\section{Factures imputées, D5.5/2-5.5.3}

REF. SOURCE: D5.5/2

REF. DESTIN.: $\quad 5.5 .3$
DESCRIPTION: Factures imputées

DESCRIPTION: Saisir les factures

\section{DESCRIPTION DÉTAILLÉE}

Factures ou comptes de dépenses ayant au minimum 2 numéros de comptes de grandlivre et des montants associés à chaque compte.

\section{STRUCTURE DE DONNÉES INCLUSES}

\section{Dans le cas de facture:}

nom du fournisseur: 60 caractères alphanumériques

adresse du fournisseur: environ 150 caractères alphanumériques

numéro de la facture: maximum 10 caractères alphanumériques

date de la facture: 8 caractères alphanumériques

montant de la facture: maximum 8 caractères numériques dont 2 décimales

description des articles commandés: environ 60 caractères alphanumériques

coût des articles commandés: maximum 7 caractères numériques dont deux décimales adresse de la facturation: environ 150 caractères alphanumériques

adresse de la livraison: environ 150 caractères alphanumériques

échéance: 25 caractères alphanumériques (généralement 30 jours)

nom de l'employé ou associé qui autorise: 30 caractères alphanumériques

initiales: maximum 3 caractères alphanumériques

numéro de compte du grand-livre ${ }^{(1)}: 4$ caractères numériques

numéro du sous-compte du grand-livre (s'il y a lieu) : 2 caractères numériques 
montant: 6 caractères numériques dont deux décimales

numéro de compte du fournisseur: 4 caractères numériques

montant: 6 caractères numériques

dans le cas de comptes de dépenses:

date: 8 caractères alphanumériques

numéro de l'employé ou de l'associé: 3 caractères numériques

nom: 40 caractères alphanumériques

approbation: nom ou initiale de la personne qui autorise

section des dépenses facturables:

code de dépense: 3 caractères numériques

numéro du client: 8 caractères numériques

5 caractères numériques pour le numéro du client, 3 caractères numériques

pour le numéro du mandat

nom du client:70 caractères alphanumériques

description de la dépense: 60 caractères alphanumériques

montant: 7 caractères numériques dont deux décimales

section des dépenses non-facturables:

code de dépense: 3 caractères numériques

description de la dépense: 60 caractères alphanumériques

montant: 7 caractères numériques dont deux décimales

VOLUME

Harel Drouin, et Associés : $350^{(1)}+100^{(2)}$

Harel Drouin Gestion Conseil $\quad: \quad 25+20$

Société d'informatique de gestion : $25+25$

Total par mois

$: 400+145=545$

1- pour les factures

2- pour les comptes de dépenses 
Flux de données

\section{Nom du fournisseur, D5-5.5.3}

REF. SOURCE: D5

REF. DESTIN.: $\quad 5.5 .3$
DESCRIPTION: Fichier maître fournisseur

DESCRIPTION: Saisir les factures

\section{DESCRIPTION DÉTAILLÉE}

Contient le nom du fournisseur ou d'un employé (dans le cas de compte de dépenses) correspondant au numéro de facture saisi.

STRUCTURE DE DONNÉES INCLUSES

nom du fournisseur: 60 caractères alphanumériques

VOLUME

Harel Drouin, et Associés

$: 350^{(1)}+100^{(2)}$

Harel Drouin Gestion Conseil

: $25+20$

Société d'informatique de gestion

Total par mois

$: 400+145=545$

1- pour les factures

2- pour les comptes de dépenses 
Flux de données

\section{Description du compte, D3-5.5.3}

REF. SOURCE: D3

REF. DESTIN.: $\quad 5.5 .3$
DESCRIPTION: Grand-livre

DESCRIPTION: Saisir les factures

\section{DESCRIPTION DÉTAILLÉE}

Contient le nom relié au numéro de compte qui a été saisi.

STRUCTURE DE DONNÉES INCLUSES

nom: 30 caractères alphanumériques

\section{VOLUME}

Harel Drouin, et Associés

$: 350^{(1)}+100^{(2)}$

Harel Drouin Gestion Conseil

$: 25+20$

Société d'informatique de gestion

: 25

$+25$

Total par mois

$: 400+145=545$

1- pour les factures

2- pour les comptes de dépenses 
Flux de données

\section{Numéro du client, D2-5.5.3}

REF. SOURCE: D2

REF. DESTIN.: $\quad 5.5 .3$
DESCRIPTION: Fichier maître client

DESCRIPTION: Saisir les facture

\section{DESCRIPTION DÉTAILLÉE}

Ce flux est employé seulement lorsque nous effectuons la saisie de comptes de dépenses. Dans le cas de dépenses facturables, le système demandera le numéro du client auquel la dépense sera imputée. Le numéro saisi sera validé avec le fichier client de Harel Drouin et associés.

STRUCTURE DE DONNÉES INCLUSES

numéro du client: 8 caractères numériques

VOLUME

Harel Drouin, et associés $: 100^{(1)}$

Harel Drouin Gestion Conseil : : 20

Société d'informatique de gestion: $\underline{25}$

Total par mois : 145 
Flux de données

\section{Écritures de journal, 5.5.3-D8}

REF. SOURCE: 5.5 .3

REF. DESTIN.: D8
DESCRIPTION: Saisir les factures

DESCRIPTION: Écritures de journal

\section{DESCRIPTION DÉTAILLÉE}

Contient le numéro de l'écriture, la date de l'écriture, le numéro de compte affecté par l'écriture, le montant de l'écriture et la description. Le numéro de l'écriture contient des caractères permettant d'identifier le type de l'écriture. Ainsi une écriture qui concernent le journal des achats peut avoir le numéro suivant: ah01; ah pour achat.

\section{STRUCTURE DE DONNÉES INCLUSES}

numéro de l'écriture: 4 caractères alphanumériques

code de transaction: 1 caractère numérique ${ }^{(1)}$

date de l'écriture : 8 caractères alphanumériques

numéro de compte: 4 caractères numériques

numéro du sous-compte (succursale) s'il y a lieu: 2 caractères numériques

montant: maximum 8 caractères numériques dont 2 décimales

description: 60 caractères alphanumériques

$\begin{array}{ll}\text { 1- code de transaction: } & \text { (1) transaction au joumal général } \\ \text { (2) transaction au joumal des revenus } \\ \text { (3) transaction au joumal de la facturation } \\ \text { (4) transaction au caisse recettes } \\ \text { (5) transaction au caise déboursés } \\ \text { (6) transaction au joumal des acahts }\end{array}$


VOLUME

Harel Drouin, et Associés : $250^{(1)}$

Harel Drouin Gestion Conseil $\quad$ : 52

Société d'informatique de gestion : 52

Total par mois $\quad$ : 354

1- Nous considérons qu'il n'y a qu'il y une écriture de journal par lot de comptes de dépenses et factures saisis. En supposant que des factures sont saisies à tous les jours pour Harel Drouin et associés, il y aura autant d'écritures que de jours ourrables. Pour les autres entreprises du groupe, le volume étant moins important, les factures seront saisies qu'une fois par semaine. 
Flux de données

\title{
Dépenses, 5.5.3-D4
}

\author{
REF. SOURCE: $5.5 .3 \quad$ DESCRIPTION: Saisir les factures \\ REF. DESTIN.: D4 DESCRIPTION: Dépenses
}

\section{DESCRIPTION DÉTAILLÉE}

Contient toutes les dépenses qui sont inscrites sur les comptes de dépenses des employés et les dépenses encourues par la corporation relatives au dossier d'un client (ex.:frais légaux, messagerie). Ces dépenses sont enregistrés dans un fichier afin d'imputer les dépenses facturables aux clients et d'enregistrer les dépenses non facturables dans le fichier du cumulatif des transactions. Les dépenses non-facturables enregistrées dans le fichier "D10 Cumulatif des transactions" permettront de contrôler, par le biais des rapports statistiques, les dépenses des employés.

\section{STRUCTURE DE DONNÉES INCLUSES}

date de la transaction: 8 caractères alphanumériques

numéro du client (dans le cas de dépense facturable):

8 caractères numériques

5 caractères numériques pour le numéro du client, 3 caractères numériques pour le numéro du mandat

montant: 7 caractères numériques dont deux décimales description de la dépense: 60 caractères alphanumériques montant: 7 caractères numériques dont deux décimales 


\section{VOLUME}

Harel Drouin, et associés $\quad: 200^{(1)}$

Harel Drouin Gestion Conseil ： $\mathbf{4 0}$

Société d'informatique de gestion: $\underline{50}$

Total par semaine $\quad: 245$ 
Flux de données

\section{Numéro d'écritures, D9-5.5.3}

REF. SOURCE: D9

REF. DESTIN.: $\quad 5.5 .3$
DESCRIPTION: Numéro d'écritures

DESCRIPTION: Saisir les factures

\section{DESCRIPTION DÉTAILLÉE}

Ce numéro est assigné par le système et sert à identifier un ensemble de transactions inscrites dans un journal et dans le grand-livre. Le numéro d'une écriture inscrit au grand-livre doit nous permettre de connaître l'origine de cette dernière. Par exemple, le numéro ah01 nous informe que nous faisons référence à une écriture qui provient du journal des achats (ah pour achat).

\section{STRUCTURE DE DONNÉES INCLUSES}

numéro de l'écriture: 4 caractères numériques

code de transaction: 1 caractère numérique ${ }^{(1)}$ 


\section{VOLUME}

Harel Drouin, et associés: maximum 999 pour chaque journal

Harel Drouin Gestion Conseil: maximum 999 pour chaque journal

Société d'informatique de gestion: maximum 999 pour chaque journal 
Flux de données

\section{Factures, 5.5.3-D6}

REF. SOURCE: 5.5 .3

REF. DESTIN.: D6
DESCRIPTION: Saisir les factures

DESCRIPTION: Auxiliaire des comptes à payer

\section{DESCRIPTION DÉTAILLÉE}

Contient toutes les comptes de dépenses et les factures reliées à des achats, des locations ou des acquisitions et envoyées par les fournisseurs. Ces comptes de dépenses et factures sont enregistrées dans l'auxiliaire des comptes à payer.

\section{STRUCTURE DE DONNÉES INCLUSES}

\section{Dans le cas de factures:}

numéro du fournisseur: 4 caractères numériques

numéro de l'écriture: 4 caractères numériques

code de transaction: 1 caractère numérique

date de la facture: 8 caractères alphanumériques

montant de la facture: maximum 8 caractères numériques dont 2 décimales description des articles commandés: généralement le numéro de la facture

Dans le cas de comptes de dépenses:

date: caractères alphanumériques

numéro de l'employé: 3 caractères numériques

montant: 7 caractères numériques dont deux décimales 
VOLUME

Harel Drouin, et Associés

$: 350^{(1)}+100^{(2)}$

Harel Drouin Gestion Conseil

$: 25+20$

Société d'informatique de gestion

$: 25+25$

Total par mois

$: 400+145=545$

1- pour les factures

2- pour les comptes de dépenses 
Flux de données

\section{Dépenses, D4-5.5.7}

REF. SOURCE: D4

REF. DESTIN.: $\quad 5.5 .7$
DESCRIPTION: Dépenses

DESCRIPTION: Imputer dépenses facturables

\section{DESCRIPTION DÉTAILLÉE}

Contient toutes les dépenses qui sont inscrites sur les comptes de dépenses des employés et les dépenses encourues par la corporation relatives au dossier d'un client (ex.: frais légaux, messagerie). Ces dépenses sont lues dans le fichier "D4 Dépenses" afin d'imputer les dépenses facturables aux clients et d'enregistrer les dépenses non-facturables dans le fichier D10 Cumulatif des transactions. Les dépenses non facturables enregistrées dans le fichier "D10 Cumulatif des transactions" permettront de contrôler, par le biais des rapports statistiques, les dépenses des employés.

\section{STRUCTURE DE DONNÉES INCLUSES}

date de la dépense: 8 caractères alphanumériques

montant: 7 caractères numériques dont deux décimales

description: 60 caractères alphanumériques

dans le cas de dépenses non facturables:

numéro de compte de dépenses (provient de la charte de compte de Harel Drouin et associés):

4 caractères numériques

dans le cas de dépenses facturable:

numéro des travaux en cours: 4 caractères numériques

numéro du client: 8 caractères numériques, 5 pour le numéro du client et 3 pour le numéro du projet 
VOLUME

Harel Drouin, et associés $\quad: 150^{(1)}$

Harel Drouin Gestion Conseil : 20

Société d'informatique de gestion: 25

Total par mois $\quad$ : 195

1- Environ 100 comptes de dépenses à 1 client par compte et cinquante dépenses facturables. 
Flux de données

\section{Écritures de journal, 5.5.7-D8}

REF. SOURCE: 5.5 .7

REF. DESTIN.: D8
DESCRIPTION: Imputer dépenses facturables

DESCRIPTION: Écritures de journal

\section{DESCRIPTION DÉTAILLÉE}

Ces écritures, qui sont enregistrées dans les fichiers contenant toutes les écritures de journal, vise à réduire les dépenses engagées par la corporation et à augmenter ses comptes à recevoir.

Pour la société, ces écritures ont pour effet d'augmenter ses dépenses et sa dette envers la corporation.

Les écritures pourraient être les suivantes:

Pour les Services Harel Drouin:

Dépenses Inter compagnie $X X$

Pour Harel Drouin et associés:

Travaux en coursXX Inter compagnie $\quad X X$

STRUCTURE DE DONNÉES INCLUSES

code de transaction: 1 caractère numérique numéro de l'écriture: 4 caractères alphanumériques date de l'écriture : 8 caractères numériques numéro de compte: 4 caractères numériques numéro du sous-compte (succursale) s'il y a lieu: 2 caractères numériques 
montant: maximum 8 caractères numériques dont 2 décimales

description: 60 caractères alphanumériques

VOLUME

Harel Drouin, et Associés : $250^{(1)}$

Harel Drouin Gestion Conseil $\quad$ : 52

Société d'informatique de gestion : $\underline{52}$

Total par mois

: 354

1- Nous considérons qu'il n’y a qu'il y une écriture de joumal par lot de comptes de dépenses et factures saisis. En supposant que des factures sont saisies à tous les jours pour Harel Drouin et associés, il y aura autant d'écritures que de jours ouvrables. Pour les autres entreprises du groupe, le volume étant moins important, les factures seront saisies qu'une fois par semaine. 
Flux de données

\section{Dépenses facturables, 5.5.7-D7}

REF. SOURCE: $\mathbf{5} .5 .7$

REF. DESTIN.: $\quad$ D7
DESCRIPTION: Imputer dépenses facturables

DESCRIPTION: Auxiliaire des travaux en cours

\section{DESCRIPTION DÉTAILLÉE}

Contient toutes les dépenses inscrites dans la sections des dépenses facturables d'un compte de dépenses d'un employé ou associé et toutes les dépenses relatives au dossier d'un client (exemple: les livraison par service de courrier) et encourues par la corporation de gestion. La corporation de gestion facturera ces dépenses à la société qui, elles les enregistrera dans son auxiliaire des travaux en cours.

\section{STRUCTURE DE DONNÉES INCLUSES}

code d'activité: 3 caractères numériques (égal au code de transaction relié aux dépenses facturables

code de transaction: 1 caractère numérique

numéro de l'écriture: 4 caractères numériques

date de la transaction: 8 caractères alphanumériques

numéro du client: 5 caractères numériques

numéro du projet: 3 caractères numériques

montant: 7 caractères numériques dont deux décimales

description de la dépense: 60 caractères alphanumériques

montant: 7 caractères numériques dont deux décimales 


\section{VOLUME}

Harel Drouin, et associés

Harel Drouin Gestion Conseil

Société d'informatique de gestion

Total par semaine
$: 52^{(1)}+52=104$

$: 52+52=104$

$: \underline{52+52=104}$

: 312

1- Les travalx en cours seront mis à jour à toutes les semaines. Donc cette opération aura pour conséquence de générer une écriture à chaque semaine. II en est de même pour Les Senvices Harel Drouin. II ne faut pas oublier qu'une écriture de journal peut affecter plusieurs comptes. 
Flux de données

\section{Dépenses non-facturables, 5.5.7-D10}

REF. SOURCE: $\mathbf{5} .5 .7$

REF. DESTIN.: D10
DESCRIPTION: Imputer dépenses facturables

DESCRIPTION: Cumulatif des transactions

\section{DESCRIPTION DÉTAILLÉE}

Contient toutes les dépenses non facturables inscrites sur les comptes de dépenses des employés ou associés. Ces dépenses devront être enregistrées dans le fichier "D10 Cumulatif des transactions" car elles doivent être contrôler par le bais de statistiques.

\section{STRUCTURE DE DONNÉES INCLUSES}

code d'activité: 3 caractères numériques

numéro de l'employé ou de l'associés: 3 caractères numériques

montant de la dépense: $\quad 6$ caractères alphanumériques dont deux décimales description de la dépense: 60 caractères alphanumériques

\section{VOLUME}

Harel Drouin, et associés $\quad: 100$

Harel Drouin Gestion Conseil : 20

Société d'informatique de gestion: $\underline{25}$

Total par semaine $\quad: 145$ 
Flux de données

\section{Date, f-5.5.4}

REF. SOURCE: $\quad f \quad$ DESCRIPTION: Personnel administratif

REF. DESTIN.: $\quad 5.5 .4 \quad$ DESCRIPTION: Imprimer liste des factures à payer

\section{DESCRIPTION DÉTAILLÉE}

Contient la date pour laquelle nous désirons imprimer la liste des factures à payer. Cette date doit être celle de la journée ou l'information est fournie ou une date ultérieure.

\section{STRUCTURE DE DONNÉES INCLUSES}

date: 8 caractères alphanumériques

\section{VOLUME}

Harel Drouin, et associés : $52^{(1)}$

Harel Drouin Gestion Conseil : 52

Société d'informatique de gestion: $\underline{52}$

Total par semaine $\quad: 156$ 
Flux de données

\section{Date, 5.5.4-D5.5/5}

$\begin{array}{llll}\text { REF. SOURCE: } & 5.5 .4 & \text { DESCRIPTION: } & \text { Imprimer liste des factures à payer } \\ \text { REF. DESTIN.: } & \text { D5.5/5 } & \text { DESCRIPTION: } & \text { Date de sélection }\end{array}$

\section{DESCRIPTION DÉTAILLÉE}

Contient la date fournie par l'employé. Cette date est la date pour laquelle nous désirons imprimer la liste des factures à payer. Elle est conservée dans un fichier car elle sera utilisée par le système pour identifier les factures dûes. Si cette date n'était pas conservée dans le système, le système demanderait une date à l'usager, la date qui serait fournie alors pourrait être différente de celle qui a été utilisée pour imprimer la liste des factures à payer et cela porterait confusion.

\section{STRUCTURE DE DONNÉES INCLUSES}

date: 8 caractères alphanumériques

\section{VOLUME}

Harel Drouin, et associés: $\quad 52^{(1)}$

Harel Drouin Gestion Conseil: $\quad 52$

Société d'informatique de gestion: $\underline{52}$

Total par semaine: $\quad 156$ 
Flux de données

\title{
Liste des factures à payer, 5.5.4-5.5.5
}

\author{
REF. SOURCE: $\quad 5.5 .4 \quad$ DESCRIPTION: Imprimer la liste des factures à payer \\ REF. DESTIN.: $\quad 5.5 .5 \quad$ DESCRIPTION: Retenir certaines factures
}

\section{DESCRIPTION DÉTAILLÉE}

Contient toutes les factures et comptes de dépenses pour lesquelles la date dûe est supérieure ou égale à la date fournie par l'usager.

\section{STRUCTURE DE DONNÉES INCLUSES}

dans le cas de factures:

numéro du fournisseur: 4 caractères numériques

nom du fournisseur: 60 caractères alphanumériques

numéro de la facture: maximum 10 caractères alphanumériques

date de la facture: 8 caractères alphanumériques

montant de la facture: maximum 8 caractères numériques dont 2 décimales

solde à payer: maximum 8 caractères numériques dont 2 décimales

échéance: 25 caractères alphanumériques (généralement $\mathbf{3 0}$ jours)

dans le cas de comptes de dépenses:

numéro de l'employé ou de l'associé: 3 caractères numériques

date: 8 caractères alphanumériques

montant: 7 caractères numériques dont deux décimales 


\section{VOLUME}

Harel Drouin, et Associés

$: 350^{(1)}+100^{(2)}$

Harel Drouin Gestion Conseil

: $25+20$

Société d'informatique de gestion

$$
\begin{aligned}
& : \frac{25+25}{: 400+145=545}
\end{aligned}
$$

Total par mois 
Flux de données

Factures, D6-5.5.4

$\begin{array}{llll}\text { REF. SOURCE: } & \text { D6 } & \text { DESCRIPTION: } & \text { Auxiliaire des comptes à payer } \\ \text { REF. DESTIN.: } & 5.5 .4 & \text { DESCRIPTION: } & \text { Imprimer la liste des factures à payer }\end{array}$

DESCRIPTION DÉTAILLÉE

Contient toutes les factures et comptes de dépenses inscrits dans l'auxiliaire des comptes à payer. La date de ces factures ou comptes de dépenses est utilisée pour calculer la date d'échéance de la facture ou du compte de dépenses.

Contient également les montants des paiements effectués sur les factures dont le solde n'est pas égal à zéro.

STRUCTURE DE DONNÉES INCLUSES

Pour les factures:

numéro du fournisseur:4 caractères numériques

numéro de la facture: maximum 10 caractères alphanumériques

date de la facture: 8 caractères alphanumériques

montant de la facture: $\quad$ maximum 8 caractères numériques dont 2 décimales

modalité de paiement: $\quad 1$ caractère alphanumérique dont la valeur peut signifier que la facture est payée dans sa totalité, que la facture est payée partiellement ou que le paiement est retenu. 
dans les cas de paiements effectués:

numéro du fournisseur: 4 caractères numériques

numéro de la facture: maximum 10 caractères alphanumériques

date de la facture: 8 caractères alphanumériques

montant du paiement: maximum 8 caractères numériques dont 2 décimales

dans le cas de comptes de dépenses:

numéro de l'employé: 3 caractères numériques

date: caractères alphanumériques

montant du compte: 7 caractères numériques dont deux décimales

VOLUME

Harel Drouin, et Associés $\quad: 350+100^{(1)}$

Harel Drouin Gestion Conseil $\quad: 25+20$

Société d'informatique de gestion : $25+25$

Total par mois $\quad: 400+145=545$ 
Flux de données

\section{Date due, D5-5.5.4}

REF. SOURCE: D5 DESCRIPTION: Fichier maître fournisseurs

REF. DESTIN.: $\quad 5.5 .4 \quad$ DESCRIPTION: $\quad$ Imprimer liste des factures à payer

\section{DESCRIPTION DÉTAILLÉE}

Contient le nombre de jours après lesquels une facture ou un comptes de dépenses devient à échéance. Cette information sert à déterminer les factures et comptes de dépenses qui doivent être payés.

STRUCTURE DE DONNÉES INCLUSES

nombre de jours: 2 caractères numériques

VOLUME

Harel Drouin, et Associés $\quad: 350+100^{(1)}$

Harel Drouin Gestion Conseil $\quad$ : $25+20$

Société d'informatique de gestion : $25+25$

Total par mois

$: 400+145=545$ 
Flux de données

\section{Date, D5.5/5-5.5.5}

REF. SOURCE: D5.5/5 DESCRIPTION: Date de sélection

REF. DESTIN.: $\quad 5.5 .5 \quad$ DESCRIPTION: Retenir certaines factures

DESCRIPTION DÉTAILLÉE

Contient la date qui a été fournie par l'usager et qui a été utilisée pour produire la liste des factures et comptes de dépenses à payer. Cette date à été conservée dans un fichier car elle sera utilisée par le système pour recalculer les factures et comptes de dépenses dûs. Si cette date n'était pas conservée dans le système, le système demanderait une date à l'usager, la date qui serait fournie alors pourrait être différente de celle qui a été utilisée pour imprimer la liste des factures et comptes de dépenses à payer et cela porterait confusion.

STRUCTURE DE DONNÉES INCLUSES

date: 8 caractères alphanumériques

\section{VOLUME}

Harel Drouin, et associés: $\quad 52^{(1)}$

Harel Drouin Gestion Conseil: $\quad 52$

Société d'informatique de gestion: $\underline{52}$

Total par semaine: $\quad 156$ 
Flux de données

\section{Factures payées, 5.5.5-D5.5/3}

$\begin{array}{llll}\text { REF. SOURCE: } & 5.5 .5 & \text { DESCRIPTION: } & \text { Retenir certaines factures } \\ \text { REF. DESTIN:: } & \text { D5.5/3 } & \text { DESCRIPTION: } & \text { Factures payées }\end{array}$

DESCRIPTION DÉTAILLÉE

Contient toutes les factures et comptes de dépenses pour lesquels un chèque sera émis.

\section{STRUCTURE DE DONNÉES INCLUSES}

Dans le cas de facture:

nom du fournisseur: 60 caractères alphanumériques

adresse du fournisseur: environ 150 caractères alphanumériques

numéro de la facture: maximum 10 caractères alphanumériques

date de la facture: 8 caractères alphanumériques

montant de la facture: $\quad$ maximum 8 caractères numériques dont 2 décimales

description des articles commandés: environ 60 caractères alphanumériques

coût des articles commandés: maximum 7 caractères numériques dont deux décimales

adresse de la facturation: environ 150 caractères alphanumériques

adresse de la livraison: environ 150 caractères alphanumériques

échéance: 25 caractères alphanumériques (généralement 30 jours)

nom de l'employé ou associé qui autorise: 30 caractères alphanumériques

initiales: maximum 3 caractères alphanumériques

numéro de compte du grand-livre ${ }^{(1)}: 4$ caractères numériques

numéro du sous-compte du grand-livre (s'il y a lieu) : 2 caractères numériques

montant: 6 caractères numériques dont deux décimales 
numéro de compte du fournisseur: 4 caractères numériques

montant: 6 caractères numériques

dans le cas de comptes de dépenses:

date: 8 caractères alphanumériques

numéro de l'employé ou de l'associé: 3 caractères numériques

section des dépenses facturables:

code de dépense: 3 caractères numériques

numéro du client: 8 caractères numériques

5 caractères numériques pour le numéro du client, 3 caractères numériques pour le numéro du mandat

nom du client:70 caractères alphanumériques

description de la dépense: 60 caractères alphanumériques

montant: 7 caractères numériques dont deux décimales

section des dépenses non-facturables:

code de dépense: 3 caractères numériques

description de la dépense: 60 caractères alphanumériques

montant: 7 caractères numériques dont deux décimales 
VOLUME

Harel Drouin, et Associés

$: 350^{(1)}+100^{(2)}$

Harel Drouin Gestion Conseil

$: 25+20$

Société d'informatique de gestion

Total par mois

$\frac{25+25}{: 400+145=545}$


Flux de données

\title{
Paiements partiels ou retenus, 5.5.5-D6
}

\author{
REF. SOURCE: $\quad 5.5 .5 \quad$ DESCRIPTION: Retenir certaines factures \\ REF. DESTIN.: D6 DESCRIPTION: Auxiliaire des comptes à payer
}

\section{DESCRIPTION DÉTAILLÉE}

Ce flux contient toute l'information nécessaire pour aller inscrire un code signifiant qu'un paiement partiel sera effectué ou que la facture sera retenue. Ce code est enregistré avec les coordonnées de la facture.

\section{STRUCTURE DE DONNÉES INCLUSES}

numéro du fournisseur: 4 caractères numériques

numéro de la facture: maximum 10 caractères alphanumériques

date de la facture: 8 caractères alphanumériques

modalité de paiement: $\quad 1$ caractère alphanumérique dont la valeur peut signifier que la facture est payée dans sa totalité, que la facture est payées partiellement ou que le paiement est retenu.

\section{VOLUME}

Harel Drouin, et Associés $\quad: 5^{(1)}$

Harel Drouin Gestion Conseil $\quad: 1$

Société d'informatique de gestion : 1

Total par mois 
Flux de données

\section{Factures payables, D6-5.5.6}

$\begin{array}{llll}\text { REF. SOURCE: } & \text { D6 } & \text { DESCRIPTION: } & \text { Auxiliaire des travaux en cours } \\ \text { REF. DESTIN.: } & 5.5 .6 & \text { DESCRIPTION: } & \text { Imprimer les chèques }\end{array}$

\section{DESCRIPTION DÉTAILLÉE}

Contient toutes les factures et comptes de dépenses pour lesquels un chèque sera émis, c'est à dire les factures et comptes de dépenses pour lesquelles la modalité de paiement signifie que la facture ou compte de dépenses sera payé entièrement ou partiellement.

\section{STRUCTURE DE DONNÉES INCLUSES}

dans le cas de factures:

numéro du fournisseur: 4 caractères numériques

numéro de la facture: maximum 10 caractères alphanumériques

date de la facture: 8 caractères alphanumériques

montant de la facture: maximum 8 caractères numériques dont 2 décimales

dans le cas de compte de dépenses:

numéro de l'employé ou de l'associé: 4 caractères numériques

date: 8 caractères alphanumériques

montant: maximum 8 caractères numériques dont 2 décimales 
VOLUME

Harel Drouin, et Associés $\quad: 350^{(1)}+100^{(2)}$

Harel Drouin Gestion Conseil $\quad: 25+20$

Société d'informatique de gestion : $25+25$

Total par mois $\quad: 400+145=545$

1-pour les factures

2-pour les comptes de dépenses 
Flux de données

\section{Montants, D5.5/6-5.5.6}

$\begin{array}{llll}\text { REF. SOURCE: } & \text { D5.5/6 } & \text { DESCRIPTION: } & \text { Paiements partiels } \\ \text { REF. DESTIN.: } & 5.5 .6 & \text { DESCRIPTION: } & \text { Imprimer les chèques }\end{array}$

DESCRIPTION DÉTAILLÉE

Contient les montants des paiements partiels de factures.

STRUCTURE DE DONNÉES INCLUSES

montant: maximum 8 caractères numériques dont 2 décimales

\section{VOLUME}

Harel Drouin, et associés: 20

Harel Drouin Gestion Conseil: $\quad 1$

Société d'informatique de gestion: 1

Total par mois: 22 
Flux de données

\section{Information sur paiement, 5.5.6-D6}

REF. SOURCE: 5.5.6 DESCRIPTION: Imprimer les chèques

REF. DESTIN.: D6 DESCRIPTION: Auxiliaire des comptes à payer

\section{DESCRIPTION DÉTAILLÉE}

Contient toute l'information relative au paiement d'une facture ou d'un compte de dépenses.

STRUCTURE DE DONNÉES INCLUSES

code de transaction: 1 caractère numérique

numéro de l'écriture: 4 caractères numériques

numéro du fournisseur: 4 caractères numériques

numéro de la facture: maximum 10 caractères alphanumériques

date du paiement: 8 caractères alphanumériques

montant du paiement: $\quad$ maximum 8 caractères numériques dont 2 décimales

\section{VOLUME}

Harel Drouin, et Associés $\quad: 350^{(1)}+100^{(2)}$

Harel Drouin Gestion Conseil $\quad$ : $25+20$

Société d'informatique de gestion $: 25+25$

Total par mois

$: 400+145=545$

1-pour les factures 
Flux de données

\section{Copies des chèques, 5.5.6-D5.5/3}

$\begin{array}{llll}\text { REF. SOURCE: } & 5.5 .6 & \text { DESCRIPTION: } & \text { Imprimer les chèques } \\ \text { REF. DESTIN.: } & \text { D5.5/3 } & \text { DESCRIPTION: } & \text { Factures payées }\end{array}$

\section{DESCRIPTION DÉTAILLÉE}

Contient toutes l'information relative aux paiements de factures ou remboursement de comptes de dépenses. Ainsi sur une copie d'un chèque, nous allons retrouver le nom et l'adresse du fournisseur ( ou de l'employé ou associé), les numéros des factures, la date des factures et le montant versé pour chaque facture couverte par le chèque (ou le compte de dépenses).

\section{STRUCTURE DE DONNÉES INCLUSES}

partie chèque:

numéro du chèque: 10 caractères numériques

nom de l'émetteur: Les services Harel Drouin inc.

adresse de l'émetteur: 507 Place d'Armes bureau 800

Montréal H2Y 2W8

montant du chèque: 7 caractères numériques dont 2 décimales

date du chèque: 8 caractères alphanumériques

nom du fournisseur: 60 caractères alphanumériques

adresse du fournisseur: 80 caractères alphanumériques

mention "non négociable"

nom de la banque: 40 caractères alphanumériques

adresse de la banque: 80 caractères alphanumériques 
partie talon:

dans le cas de facture:

numéro des factures couvertes par le chèque: pour chaque numéro, maximum 10 caractères alphanumériques

date des factures: pour chaque facture, 8 caractères alphanumériques

montant des paiements: 7 caractères numériques

dans le cas de compte de dépenses:

date du compte de dépenses: 8 caractères alphanumériques

montant: 7 caractères numériques

VOLUME

Harel Drouin, et Associés $\quad: 350^{(1)}+100^{(2)}$

Harel Drouin Gestion Conseil $\quad$ : $25+20$

Société d'informatique de gestion : $25+25$

Total par mois $\quad: 400+145=545$

1- pour les factures

2- pour les comptes de dépenses 
Flux de données

\section{Écritures de journal, 5.5.6-D8}

REF. SOURCE: $\quad 5.5 .6 \quad$ DESCRIPTION: Imprimer les chèques

REF. DESTIN.: D8 DESCRIPTION: Écritures de journal

\section{DESCRIPTION DÉTAILLÉE}

Contient une écriture affectant les comptes à payer et la banque qui concerne l'enregistrement du paiement de certaines factures et comptes de dépenses.

\section{STRUCTURE DE DONNÉES INCLUSES}

code de transaction: 1 caractère numérique

numéro de l'écriture: 4 caractères alphanumériques

date de l'écriture : 6 caractères numériques

numéro de compte: 4 caractères numériques

numéro du sous-compte (succursale) s'il y a lieu: 2 caractères numériques

montant: maximum 8 caractères numériques dont 2 décimales

description: 60 caractères alphanumériques

VOLUME

Harel Drouin, et Associés : $52^{(1)}$

Harel Drouin Gestion Conseil $\quad$ : 52

Société d'informatique de gestion : $\underline{52}$

Total par mois 
Flux de données

\title{
Chèques, 5.5.6-d
}

\author{
REF. SOURCE: $\quad 5.5 .6 \quad$ DESCRIPTION: Imprimer les chèques \\ REF. DESTIN.: d DESCRIPTION: Fournisseurs
}

\section{DESCRIPTION DÉTAILLÉE}

Contient toute l'information relative aux paiements de factures. Ainsi sur un chèque, nous allons retrouver le nom et l'adresse du fournisseur, les numéros des factures et le montant versées pour chaque facture couverte par le chèque.

\section{STRUCTURE DE DONNÉES INCLUSES}

partie chèque:

numéro du chèque: 10 caractères numériques

nom de l'émetteur: Les services Harel Drouin inc.

adresse de l'émetteur: 507 Place d'Armes bureau 800

Montréal H2Y 2W8

montant du chèque: 7 caractères numériques dont 2 décimales

date du chèque: 8 caractères alphanumériques

nom du fournisseur: 60 caractères alphanumériques

adresse du fournisseur: 80 caractères alphanumériques

mention "non négociable"

nom de la banque: $\mathbf{4 0}$ caractères alphanumériques

adresse de la banque: 80 caractères alphanumériques 
partie talon:

numéro des factures couvertes par le chèque: maximum 10 caractères alphanumériques date des factures: pour chaque date, 8 caractères alphanumériques montant des paiements: pour chaque montant, 7 caractères numériques

VOLUME

Harel Drouin, et Associés $\quad: 350^{(1)}$

Harel Drouin Gestion Conseil $\quad$ : 25

Société d'informatique de gestion : 25

Total par mois $\quad: 400$ 
Flux de données

\section{Chèques, 5.5.6-b}

$\begin{array}{llll}\text { REF. SOURCE: } & 5.5 .6 & \text { DESCRIPTION: } & \text { Imprimer les chèques } \\ \text { REF. DESTIN.: } & \text { b } & \text { DESCRIPTION: } & \text { Membre du personnel }\end{array}$

\section{DESCRIPTION DÉTAILLÉE}

Contient toute l'information relative aux remboursements de comptes de dépenses. Ainsi sur un chèque, nous allons retrouver le nom et l'adresse de l'employé, le mois du compte de dépenses et le montant versé.

\section{STRUCTURE DE DONNÉES INCLUSES}

partie chèque:

numéro du chèque: 10 caractères numériques

nom de l'émetteur: Les services Harel Drouin inc.

adresse de l'émetteur: 507 Place d'Armes bureau 800

Montréal H2Y 2W8

montant du chèque: 7 caractères numériques dont 2 décimales

date du chèque: 8 caractères alphanumériques

nom de l'employé ou de l'associé: 60 caractères alphanumériques

adresse de l'employé ou de l'associé: 80 caractères alphanumériques

mention "non négociable"

nom de la banque: $\mathbf{4 0}$ caractères alphanumériques

adresse de la banque: 80 caractères alphanumériques

partie talon:

date du compte de dépenses: 8 caractères alphanumériques

montant: 7 caractères numériques 


\section{VOLUME}

Harel Drouin, et Associés $\quad: 100^{(1)}$

Harel Drouin Gestion Conseil $\quad$ : 20

Société d'informatique de gestion : 25

Total par mois $\quad: 145$

1. Contient que les chèques associés au remboursement de comptes de dépenses. 
Flux de données

\section{Coordonnées, D5-5.5.6}

REF. SOURCE: D5 DESCRIPTION: Fichier maître fournisseurs

REF. DESTIN.: $\quad 5.5 .6 \quad$ DESCRIPTION: Imprimer les chèques

\section{DESCRIPTION DÉTAILÉE}

Contient le nom et l'adresse du fournisseur ou de l'employé correspondant au numéro du fournisseur. Le numéro du fournisseur est numéro de compte dans l'auxiliaire des comptes à payer.

\section{STRUCTURE DE DONNÉES INCLUSES}

nom du fournisseur: 60 caractères alphanumériques

adresse du fournisseur: environ 150 caractères alphanumériques

VOLUME

Harel Drouin, et Associés

$: 350^{(1)}+100^{(2)}$

Harel Drouin Gestion Conseil

: $25+20$

Société d'informatique de gestion

$25+25$

Total par mois

$: 400+145=545$ 
Flux de données

\section{Nom du fournisseur, D5-5.5.4}

REF. SOURCE: D5 DESCRIPTION: Fichier maître fournisseurs

REF. DESTIN.: $\quad 5.5 .4 \quad$ DESCRIPTION: Imprimer liste des factures à payer

DESCRIPTION DÉTAILLÉE

Contient le nom du fournisseur qui sera imprimée dans la liste des factures à payer. Le nom du fournisseur qui sera inscrit correspond au numéro du fournisseur inscrit dans l'auxiliaire des comptes à payer.

STRUCTURE DE DONNÉES INCLUSES

nom du fournisseur: 60 caractères alphanumériques

VOLUME

Harel Drouin, et Associés

$: 350^{(1)}+100^{(2)}$

Harel Drouin Gestion Conseil

: $25+20$

Société d'informatique de gestion

$: 25+25$

Total par mois

$: 400+145=545$

1- pour les factures

2- pour les comptes de dépenses 
Flux de données

\title{
Information fournisseurs, 5.5.8-D5
}

\author{
REF. SOURCE: $\quad 5.5 .8 \quad$ DESCRIPTION: Enregistrer les nouveaux fournisseurs \\ REF. DESTIN.: D5 DESCRIPTION: Fichier maître fournisseurs
}

\section{DESCRIPTION DÉTAILLÉE}

Contient le nom, l'adresse du fournisseur et les modalités de paiement.

\section{STRUCTURE DE DONNÉES INCLUSES}

nom du fournisseur: 60 caractères alphanumériques

adresse du fournisseur: environ 150 caractères alphanumériques

modalité de paiement: le nombre de jours après lesquels une facture doit être payées.

Généralement, 30 jours. 


\section{Processus 5.6, Produire les états financiers}


Diagramme de flux de données 
5.6 Produire les états financiers

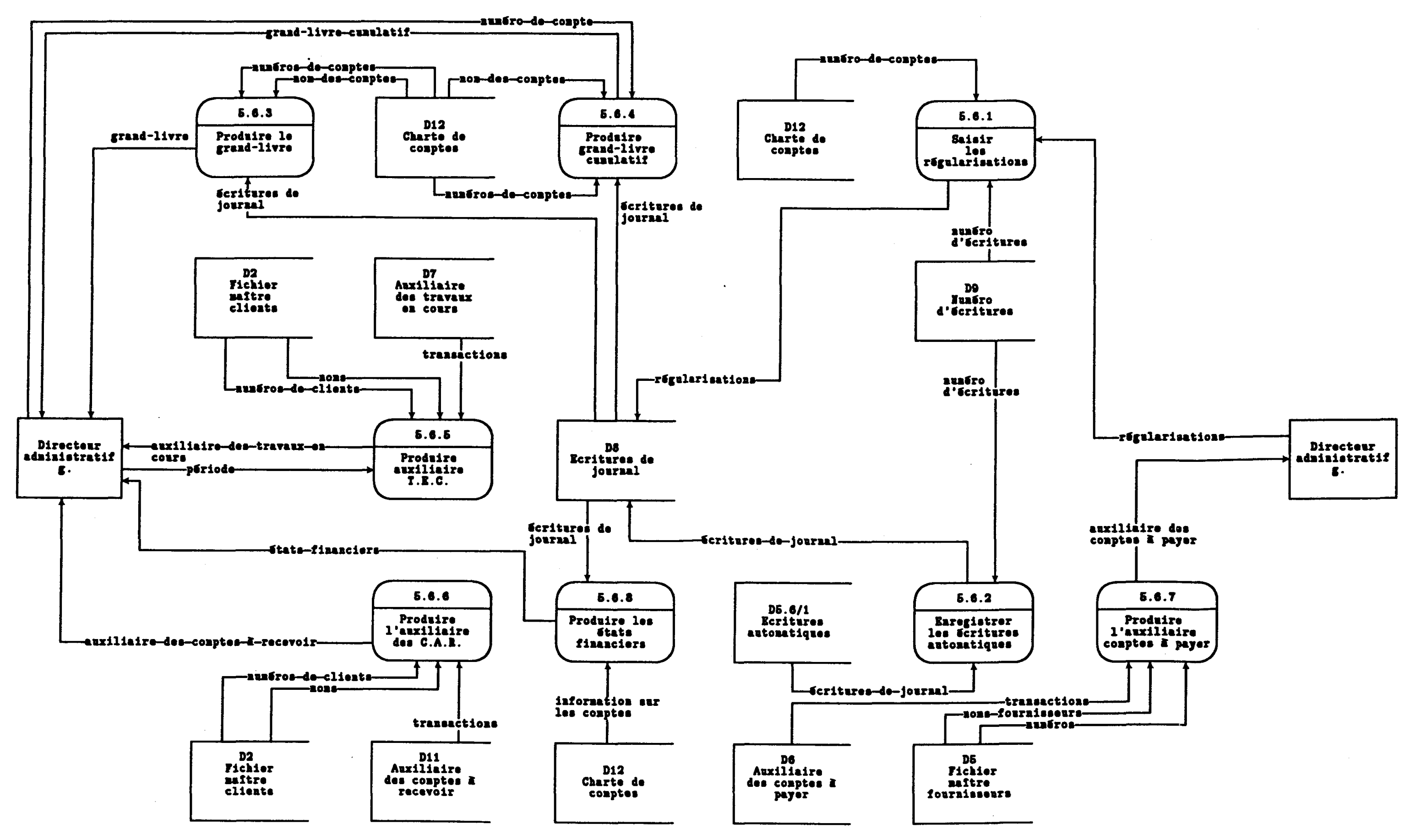


Processus de traitement 
Processus de traitement

\section{Saisir les régularisations, $\mathbf{5 . 6 . 1}$}

\section{DESCRIPTION}

Ce processus vise à enregistrer les écritures qui apporteront des modifications aux états financiers. Ces écritures, qui sont des écritures de journal, doivent apparaître, lorsque l'on fait imprimer les journaux, dans le journal général. Le système attribuera automatiquement un numéro d'écriture. Ce numéro sera employé par le système pour identifier le journal auquel les écritures se rapportent.

Le système doit permettre d'enregistrer les écritures de régularisations dans n'importe quelle période de l'année financière.

\section{ENTRÉE}

régularisations, g-5.6.1

numéro de comptes, D12-5.6.1

numéro d'écritures, D9-5.6.1

\section{SORTIE}

régularisations, 5.6.1-D9

\section{RÉSUMÉ DE LA LOGIQUE}

\section{A l'écran:}

enregistrer la date de la régularisation

tant que le numéro de compte n'est pas valide enregistrer le numéro de compte

vérifier, dans la charte de compte, l'existence du numéro de compte saisi

si le numéro n'existe pas afficher message d'erreur

tant que le numéro du sous-compte n'est pas valide enregistrer le numéro du sous-compte vérifier, dans la charte de compte, l'existence du numéro du sous-compte saisi si le numéro n'existe pas afficher message d'erreur

enregistrer le montant de la régularisation la description

Lire, dans le fichier D9 Numéro d'écritures, le numéro de l'écriture

Inscrire, dans le fichier D8 Écritures de journal: la date de la régularisation

le numéro de l'écriture 
le code de transaction

le numéro de compte

le numéro du sous-compte, s'il y a lieu

le montant de la régularisation

la description 
Processus de traitement

\section{Enregistrer les écritures automatiques, 5.6.2}

\section{DESCRIPTION}

Ce processus visent à enregistrer, dans le fichier D8 Écritures de journal, les écritures automatiques. Les écritures automatiques sont des écritures qui se répètent mensuellement. Leur objectif est d'enregistrer les dépenses d'amortissement, la dépense de mauvaises créances etc.. Elles sont inscrites dans un fichier au début de l'année financière.

\section{ENTRÉE}

numéro d'écritures, D9-5.6.2

écritures de journal, D5.6/1-5.6.2

\section{SORTIE}

Écritures de journal, 5.6.2-D8

\section{RÉSUMÉ DE LA LOGIQUE}

Pour toutes les écritures enregistrées dans le fichier D5.6/1 Écritures automatiques Lire:

le numéro du compte de grand-livre affecté par l'écriture

le numéro du sous-compte s'il y a lieu

le montant de l'écriture

la description de l'écriture

Lire, dans le fichier D9 Numéro d'écriture, le numéro de l'écriture

Inscrire, dans le fichier D8 Ecritures de journal:

la date de l'écriture

le numéro de l'écriture

le code de transaction

le numéro de compte

le numéro du sous-compte, s'il y a lieu

le montant de l'écriture

la description 
Processus de traitement

\section{Produire le grand-livre, 5.6.3}

\section{DESCRIPTION}

Ce processus vise à produire un document qui contient toutes les transactions ayant affectées les comptes du grand-livre. Ce processus calcule le solde du compte au début du mois, imprime les transactions du mois et calcule le solde à la fin du mois et ce, pour tous les comptes du grand-livre. Pour remplir cette tâche, le processus doit trier les transactions de façon à ce quelles soient classées par ordre numérique de compte.

\section{ENTRÉE}

écritures de journal, D8-5.6.3

nom des comptes, D12-5.6.3

numéros de comptes, 5.6.3-D12

\section{SORTIE}

grand-livre, 5.6.3-g

\section{RÉSUMÉ DE LA LOGIQUE}

Mettre le cumulatif égal à zéro

Trier toutes les écritures de journal dans l'ordre numérique de numéro de compte et dans l'ordre chronologique de date.

Imprimer l'entête du rapport et la date du système

Demander à l'usager pour quelle date il désire le grand-livre

Pour chaque compte inscrit dans la charte de compte

Pour toutes les écritures de journal

inscrire le numéro et le nom du compte sur le rapport

Pour toutes les écritures contenant ce numéro de compte

lire une écriture de journal

regarder si le mois de l'écriture est inférieur au mois inclut dans la date fournie par l'usager

si oui,

additionner le montant de l'écriture au cumulatif sinon,

imprimer le contenu de l'écriture

additionner le montant de l'écriture au cumulatif

changer d'écriture

inscrire le cumulatif sur la rapport

remettre le cumulatif à zéro

Changer de numéro de compte 
Processus de traitement

\section{Produire le grand-livre cumulatif, 5.6.4}

\section{DESCRIPTION}

L'impression du grand-livre cumulatif consiste à imprimer, pour tous les comptes inscrit dans la charte de compte, toutes les transactions et à imprimer le solde de chaque compte.

\section{ENTRÉE}

numéro de comptes, D12-5.6.4

nom des comptes, D12-5.6.4

écribures de journal, D8-5.6.4

numéro de compte, g-5.6.4
SORTIE

grand-live cumulatif, $5.6 .4-\mathrm{g}$

\section{RÉSUMÉ DE LA LOGIQUE}

Mettre le cumulatif égal à zéro

Trier toutes les écritures de journal dans l'ordre numérique de numéro de compte et dans l'ordre chronologique de date.

Demander à l'usager s'il désire imprimer son rapport

si oui,

Imprimer l'entête du rapport et la date du système

Pour chaque compte inscrit dans la charte de compte

Pour toutes les écritures de joumal

inscrire le numéro et le nom du compte sur le rapport

Pour toutes les écritures contenant ce numéro de compte lire une écriture de journal additionner le montant de l'écriture au cumulatif imprimer le contenu de l'écriture

Changer d'écriture

inscrire le cumulatif sur la rapport

remettre le cumulatif à zéro

sinon,

Changer de numéro de compte

demander à l'usager le numéro du compte qu'il désire consulter afficher les transactions ayant le numéro de compte saisi

afficher le solde du compte 
Processus de traitement

\section{Produire auxiliaire des T.E.C., 5.6.5}

\section{DESCRIPTION}

L'impression de l'auxiliaire des travaux en cours consiste à imprimer toutes les transactions ayant affecté les comptes clients, et ce pour un mois donné. Le système permettra également de produire, sur demande, l'ensemble des transactions pour tous les clients, pour plusieurs clients ou un seul. Lorsque l'auxiliaire est imprimé pour une période donnée, le système calculera le solde des comptes de chaque clients au début de la période, inscrira chaque transaction ayant modifié le compte client et calculera le solde à la fin de la période.

\section{ENTRÉE}

période, g-5.6.5

transactions, D7-5.6.5

numéros de clients, D2-5.6.5

nom, D2-5.6.5
SORTIE

Auxiliaire des travaux en cours, $5.6 .5-\mathrm{g}$

\section{RÉSUMÉ DE LA LOGIQUE}

Mettre le cumulatif clients égal à zéro

Mettre le cumulatif auxiliaire des travaux en cours égal à zéro

Trier toutes les transactions de l'auxiliaire des travaux en cours dans l'ordre numérique de clients et dans l'ordre de date.

Imprimer l'entéte du rapport et la date du système.

Demander à l'usager pour quel date il désire son rapport.

Pour tous les clients inscrits dans le fichier maitre clients

Lire une transactions

Pour toutes les transactions inscrites dans l'auxiliaire des travaux en cours

Inscrire le numéro et le nom du client sur le rapport

Regarder si la date de la transaction est inférieure à la date fournie par l'usager

si oui, additionner le montant de la transaction au cumulatif clients additionner le montant de la transaction au cumulatif travaux en cours sinon, imprimer le contenu de la transaction additionner le montant de la transaction au cumulatif clients additionner le montant de la transaction au cumulatif travaux en cours 
Changer de transaction

Si le client est différent du précédent

Inscrire le cumulatif client sur le rapport

Remettre le cumulatif client égal à zéro

Inscrire le numéro et le nom du client sur le rapport

Imprimer, sur le rapport, le cumulatif auxiliaire travaux en cours. 
Processus de traitement

\section{Produire l'auxiliaire des C.A.R., 5.5.6}

\section{DESCRIPTION}

L'impression de l'auxiliaire des comptes à recevoir consiste à imprimer toutes les factures ayant modifié les comptes clients. Le système permettra également de produire, sur demande, rensemble des factures pour tous les clients, pour plusieurs clients ou un seul. Lorsque l'auxiliaire est imprimé pour une période donnée, le système calculera le solde des comptes de chaque client au début de la période, inscrira chaque facture ayant modifié le compte client et calculera le solde à la fin de la période.

\section{ENTRÉE}

numéros de clients, D2-5.6.6

noms, D2-5.6.6

transactions, D11-5.6.6

\section{SORTIE}

auxiliaire des comptes à recevoir, 5.6.6-g

\section{RÉSUMÉ DE LA LOGIQUE}

Mettre le cumulatif clients égal à zéro

Mettre le cumulatif auxiliaire des comptes à recevoir égal à zéro

Trier toutes les transactions de l'auxiliaire des comptes à recevoir dans l'ordre numérique de clients, dans l'ordre de numéro de référence et dans l'ordre de date.

Imprimer l'entête du rapport et la date du système.

Demander à l'usager pour quel date il désire son rapport.

Pour tous les clients inscrit dans le fichier maître clients

Lire une transaction

Pour toutes les transactions inscrites dans l'auxiliaire des comptes à recevoir additionner le montant de la transaction au cumulatif clients additionner le montant de la transaction au cumulatif comptes à recevoir Inscrire le numéro et le nom du client sur le rapport

Lire la transaction suivante

Regarder si les transactions ont le même numéro de référence

si oui, additionner ensemble le montant des transactions

si le solde est différents de zéro

sinon

imprimer les deux transactions

imprimer la première transaction 
additionner le montant de la transaction au cumulatif comptes à recevoir Changer de transaction

Si le client est différent du précédent inscrire le numéro et le nom du client sur le rapport Inscrire le cumulatif client sur le rapport

Remettre le cumulatif client égal à zéro

Imprimer, sur le rapport, le cumulatif auxiliaire comptes à recevoir. 
Processus de traitement

\section{Produire l'auxiliaire comptes à payer, 5.6.7}

\section{DESCRIPTION}

L'impression de l'auxiliaire des comptes à payer consiste à imprimer toutes les transactions ayant affecté les comptes fournisseurs et ce pour un mois donné. Le système permettra également de produire, sur demande, l'ensemble des transactions pour tous les fournisseurs, pour plusieurs fournisseurs ou un seul. Lorsque l'auxiliaire est imprimé pour une période donnée, le système calculera le solde des comptes de chaque fournisseur au début de la période, inscrira chaque transaction ayant modifié le compte fournisseur et calculera le solde à la fin de la période.

\section{ENTRÉE}

transactions, D6-5.6.7

numéros, D5-5.6.7

nom fournisseurs, D5-5.6.7

\section{SORTIE}

auxiliaire des comptes à payer, 5.6.7-g

\section{RÉSUMÉ DE LA LOGIQUE}

Mettre le cumulatif fournisseurs égal à zéro

Mettre le cumulatif auxiliaire des comptes à payer égal à zéro

Trier toutes les transactions de l'auxiliaire des comptes à payer dans l'ordre numérique de clients et dans l'ordre de date.

Imprimer l'entête du rapport et la date du système.

Pour tous les fournisseurs inscrits dans le fichier maître fournisseurs

Lire une transaction

Pour toutes les transactions inscrites dans l'auxiliaire des comptes à payer

Inscrire le numéro et le nom du fournisseur sur le rapport

Additionner le montant de la transaction au cumulatif fournisseurs

Additionner le montant de la transaction au cumulatif comptes à payer

Lire la transaction suivante

Regarder si les transactions ont le même numéro de référence

si oui,

additionner ensemble le montants des transactions

si le solde est différent de zéro

imprimer les deux transactions sinon 
additionner le montant de la transaction au cumulatif des comptes à payer Changer de transaction

Si le fournisseur est différent du précédent

inscrire le numéro et le nom du fournisseur sur le rapport

Inscrire le cumulatif fournisseur sur le rapport

Remettre le cumulatif foumisseur égal à zéro

Imprimer, sur le rapport, le cumulatif auxiliaire comptes à payer. 
Processus de traitement

\section{Produire les états financiers, 5.6.8}

\section{DESCRIPTION}

La production des états financiers visent à compiler l'ensemble des opérations de l'entreprise dans le but de produire un document représentant l'image de l'entreprise à une période précise dans l'année. Dans le cas des entreprises du groupe Harel Drouin, la fin de l'année fiscale est le 31 janvier.

Pour produire les états financiers, on additionne, au solde du début, l'ensemble des transactions ayant affecté chaque compte du grand-livre. On regroupe également ensemble, plusieurs solde afin d'obtenir des regroupements de postes. Ainsi, par exemple, les soldes des comptes de l'équipement de bureau, de l'équipement informatique sont additionnés ensemble pour produire, dans le bilan, le poste équipement.

\section{ENTRÉE}

écritures de journal, D8-5.6.8

information sur les comptes, D12-5.6.8
SORTIE

états financiers, 5.6.8-g 


\section{RÉSUMÉ DE LA LOGIQUE}

Trier toutes les écritures de journal dans l'ordre de numéro de compte, de date, de code de transactions, de numéro d'écritures

Mettre le total de l'actif à court terme égal à zéro

Mettre le total de l'actif à long terme égal à zéro

Mettre le total de l'actif égal à zéro

Mettre le total du passif à court terme égal à zéro

Mettre le total du passif à long terme égal à zéro

Mettre le total de l'avoir des sociétaires égal à zéro

Mettre le total du passif et de l'avoir des sociétaires égal à zéro

Écrire l'entête du rapport financier

Pour tous les comptes du bilan inclus dans la charte de compte

Lire le numéro du compte

Lire le nom du compte

Lire la sorte de compte (i)

Lire le type de compte ${ }^{(2)}$

Tant qu'il s'agit de la même sorte de compte

Si le type du compte signifie que le compte est un compte d'entête Inscrire le nom du compte sur le rapport

Si le type du compte signifie que le compte regroupe plusieurs comptes

Pour tous les comptes compris dans le regroupement

Inscrire le nom du compte sur le rapport

Additionner tous les soldes du début et toutes les écritures qui ont affecté les comptes inclus dans le regroupement

Inscrire le total obtenu sur le rapport

Si le compte est un compte d'actif à court terme

additionner le total obtenu au total de l'actif à court terme

Si le compte est un compte d'actif à long terme

additionner le total obtenu au total de l'actif à long terme

Si le compte est un compte d'actif

additionner le total obtenu au total de l'actif

Si le compte est un compte de passif à court terme

additionner le total obtenu au total du passif à court terme

Si le compte est un compte de passif à long terme

additionner le total obtenu au total du passif à long terme

Si le compte est un compte de l'avoir des sociétaire

additionner le total obtenu au total de l'avoir des sociétaires

Si le compte est un compte de passif ou un compte de l'avoir des sociétaires additionner le total obtenu au total du passif et de l'avoir des sociétaires

Changer de compte

S'il ne s'agit pas de la méme sorte de compte

Inscrire le total obtenu

Remettre le total égal à zéro

1- Compte d'actif à court terme

Compte d'actit à long terme

Compte de passif a court terme

Compte de passif à long terme

Compte de lavoir des sociétaires etc.

2- Compte d'entête

Compte qui regroupe plusieurs comptes etc. 
Flux de données 
Flux de données

Régularisations, g-5.6.1

REF. SOURCE: $g$ DESCRIPTION: Directeur administratif

REF. DESTIN.: $\quad 5.6 .1 \quad$ DESCRIPTION: Saisir les régularisations

\section{DESCRIPTION DÉTAILLEEE}

Une écriture de régularisation est une écriture visant à corriger le solde d'un ou de plusieurs comptes du grand-livre. Généralement ces écritures sont enregistrés mensuellement ou annuellement. Pour faciliter l'enregistrement des écritures, nous inscrivons ces demières sur une feuille de transactions.

\section{STRUCTURE DE DONNÉES INCLUSES}

date de l'écriture : 8 caractères alphanumériques

numéro de compte: 4 caractères numériques

numéro du sous-compte (succursale) s'il y a lieu: 2 caractères numériques

montant: maximum 8 caractères numériques dont 2 décimales

description: 60 caractères alphanumériques

\section{VOLUME}

Harel Drouin, et Associés: très variable, mais le nombre ne peut dépasser 30 écritures par mois.

Harel Drouin Gestion Conseil: $\quad$ variable

Société d'informatique de gestion: variable

Total par mois:

variable 
Flux de données

\section{Numéro de comptes, D12-5.6.1}

REF. SOURCE: D12 DESCRIPTION: Chartes de comptes

REF. DESTIN.: $\quad 5.6 .1 \quad$ DESCRIPTION: Saisir les régularisations

DESCRIPTION DÉTAILÉE

Contient les numéros des comptes de grand-livre. Peut également contenir des numéros de sous-comptes. Pour chaque écritures de régularisations saisies, le numéro de compte et de sous-compte s'il y a lieu est validé.

STRUCTURE DE DONNÉES INCLUSES

numéro de compte: 4 caractères numériques

numéro du sous-compte (succursale) s'il y a lieu: 2 caractères numériques

VOLUME

Harel Drouin, et Associés: $\quad$ très variable, mais le nombre ne peut dépasser 30 écritures par mois.

Harel Drouin Gestion Conseil: $\quad$ variable

Société d'informatique de gestion: variable

Total par mois: $\quad$ variable 
Flux de données

\section{Numéro d'écritures, D9-5.6.1}

REF. SOURCE: D9 DESCRIPTION: Numéro d'écritures

REF. DESTIN.: $\quad 5.6 .1 \quad$ DESCRIPTION: Saisir les régularisations

\section{DESCRIPTION DÉTAILLÉE}

C'est un numéro qui est assigné automatiquement par le système. Ce numéro permet de retracer, au grand-livre, l'origine (1) de l'écriture.

\section{STRUCTURE DE DONNÉES INCLUSES}

numéro de l'écriture: 4 caractères alphanumériques

code de transaction: 1 caractère numérique (2)

1- Dans le cas d'écritures de régularisation, l'origine est toujours le joumal général.

(1) transaction au joumal général

(2) transaction au joumal des revenus

(3) transaction au joumal de la facturation

(4) transaction au caisse recottes

(5) transaction au caise déboursés

(6) transaction au joumal des achats 


\section{VOLUME}

Harel Drouin, et Associés: $\quad$ très variable, mais le nombre ne peut dépasser 30 écritures par mois.

Harel Drouin Gestion Conseil: $\quad$ variable

Société d'informatique de gestion: variable

Total par mois: $\quad$ variable 
Flux de données

\section{Régularisations, 5.6.1-D8}

$\begin{array}{llll}\text { REF. SOURCE: } & 5.6 .1 & \text { DESCRIPTION: } & \text { Saisir les régularisations } \\ \text { REF. DESTIN.: } & \text { D8 } & \text { DESCRIPTION: } & \text { Écritures de journal }\end{array}$

\section{DESCRIPTION DÉTAILLÉE}

Une écriture de régularisation est une écriture visant à corriger le solde d'un ou de plusieurs comptes du grand-livre. Généralement ces écritures sont enregistrées mensuellement ou annuellement.

\section{STRUCTURE DE DONNÉES INCLUSES}

numéro de l'écriture: 4 caractères alphanumériques

code de transaction: 1 caractère numérique (1)

date de l'écriture : 8 caractères alphanumériques

numéro de compte: 4 caractères numériques

numéro du sous-compte (succursale) s'il y a lieu: 2 caractères numériques

montant: maximum 8 caractères numériques dont 2 décimales

description: 60 caractères alphanumériques

1. code de transaction:

(1) transaction au joumal général

(2) transaction au joumal des revenus

(3) transaction au joumal do la facturation

(4) transaction au caisse recottes

(5) transaction au caise déboursés

(6) transaction au joumal des achats 


\section{VOLUME}

Harel Drouin, et Associés: $\quad$ très variable, mais le nombre ne peut dépasser 30 écritures par mois.

Harel Drouin Gestion Conseil: $\quad$ variable

Société d'informatique de gestion: variable

Total par mois: $\quad$ variable 
Flux de données

\section{Écritures de journal, D5.6/1-5.6.2}

REF. SOURCE: D5.6/1DESCRIPTION: Écritures automatiques

REF. DESTIN.: $\quad 5.6 .2 \quad$ DESCRIPTION: Enregistrer les écritures automatiques

DESCRIPTION DÉTAILLÉE

Les écritures de journal sont des écritures qui ressemblent grandement aux écritures de régularisations. Nous les avons nommées écritures de journal" parce qu'elles apparaîtront dans un journal spécial. Les écritures de journal auxquelles nous faisons référence ici sont des écritures répétitives qui sont saisies qu'une seule fois dans le système informatique. Généralement, ces écritures concernent l'amortissement.

STRUCTURE DE DONNÉES INCLUSES

numéro de compte: 4 caractères numériques

numéro du sous-compte (succursale) s'il y a lieu: $\mathbf{2}$ caractères numériques

montant: maximum 8 caractères numériques dont 2 décimales

description: 60 caractères alphanumériques 
VOLUME

Harel Drouin, et Associés: $\quad$ variable, mais le nombre ne peut dépasser 30 écritures par mois.

Hared Drouin Gestion Conseil: variable

Société d'informatique de gestion: variable

Total par mois: variable 
Flux de données

\title{
Écritures de journal, 5.6.2-D8
}

\author{
REF. SOURCE: $\quad 5.6 .2$ DESCRIPTION: Enregistrer écritures automatiques \\ REF. DESTIN.: D8 DESCRIPTION: Écritures de journal
}

\section{DESCRIPTION DÉTAILLÉE}

Ces écritures proviennent du fichier D5.6/1 Écritures automatiques et servent à enregistrer des écritures de journal répétitives. Généralement, ces écritures concernent l'amortissement.

\section{STRUCTURE DE DONNÉES INCLUSES}

numéro de l'écriture: 4 caractères alphanumériques

code de transaction: 1 caractère numérique (1)

date de l'écriture : 8 caractères alphanumériques

numéro de compte: 4 caractères numériques

numéro du sous-compte (succursale) s'il y a lieu: 2 caractères numériques

montant: maximum 8 caractères numériques dont 2 décimales

description: 60 caractères alphanumériques

1- code de transaction:

(1) transaction au joumal général

(2) transaction au joumal des rovenus

(3) transaction au joumal de la facturation

(4) transaction au caisso recottes

(5) transaction au caise déboursés

(6) transaction au joumal des achats 
VOLUME

Harel Drouin, et Associés: $\quad$ variable, mais le nombre ne peut dépasser 30 écritures par mois.

Harel Drouin Gestion Conseil: $\quad$ variable

Société d'informatique de gestion: variable

Total par mois: $\quad$ variable 
Fiux de données

\section{Grand-livre, 5.6.3-g}

REF. SOURCE: $\quad 5.6 .3 \quad$ DESCRIPTION: Produire le grand-livre

REF. DESTIN.: $\quad$ g $\quad$ DESCRIPTION: Directeur administratif

\section{DESCRIPTION DÉTAILLÉE}

Le grand-livre contient tous les transactions du mois ayant modifié la situation financière des entreprises du groupe Harel Drouin. Ces transactions sont regroupées par comptes et nous retrouvons deux catégories de comptes: les comptes d'opérations et les comptes de bilan.

STRUCTURE DE DONNÉES INCLUSES

pour chaque compte du grand-livre:

le solde du début du mois

toutes les écritures de journal ayant affecté le compte

les solde de la fin

\section{VOLUME}

Harel Drouin, et associés: 1

Harel Drouin Gestion Conseil: $\quad 1$

Société d'informatique de gestion: 1

Total par mois: 3 
Flux de données

\section{Numéros de comptes, D12-5.6.3}

REF. SOURCE.: D12 DESCRIPTION: Charte de comptes

REF. DESTIN.: $\quad 5.6 .3 \quad$ DESCRIPTION: Produire le grand-livre

DESCRIPTION DÉTAILLEEE

Ce flux de données contient tous les numéros de comptes inscrit dans la charte de compte. Il est employé pour imprimer le grand-livre. Pour chaque compte inscrit dans la charte de compte, le logiciel calcule le solde au début du mois, imprime les transactions du mois et calcule le solde à la fin du mois.

STRUCTURE DE DONNÉES INCLUSES

numéro: 4 caractères numériques

\section{VOLUME}

Harel Drouin, et associés: environ 200

Harel Drouin Gestion Conseil: environ 200

Société d'informatique de gestion: environ 200

Total par semaine: environ 600 
Flux de données

\title{
Nom des comptes, D12-5.6.3
}

\author{
REF. SOURCE: D12 DESCRIPTION: Charte de comptes \\ REF. DESTIN.: $\quad 5.6 .3 \quad$ DESCRIPTION: Produire grand-livre
}

\section{DESCRIPTION DÉTAILÉE}

Contient le nom de comptes correspondant aux numéros des comptes qui ont été lus dans la charte de comptes. Le nom de chaque compte est imprimé à coté du numéro de compte. Le tout est suivis des transactions ayant affecté le compte durant le mois.

STRUCTURE DE DONNÉES INCLUSES

nom: $\mathbf{4 0}$ caractères alphanumériques

\section{VOLUME}

Harel Drouin, et associés: environ 200

Harel Drouin Gestion Conseil: $\quad$ environ $\mathbf{2 0 0}$

Société d'informatique de gestion: environ 200

Total par semaine: environ 600 
Flux de données

\section{Écritures de journal, D8-5.6.3}

$\begin{array}{llll}\text { REF. SOURCE: } & \text { D8 } & \text { DESCRIPTION: } & \text { Écritures de joumal } \\ \text { REF. DESTIN.: } & 5.6 .3 & \text { DESCRIPTION: } & \text { Produire grand-livre }\end{array}$

DESCRIPTION DÉTAILÉE

Ce flux contient l'ensemble des transactions ayant modifié la situation financière des entreprises du groupe Harel Drouin. Pour chaque compte (autre que les comptes titre et regroupement) inscrit dans la charte de compte:

- les transactions ayant affecté le solde du compte sont lues

- les montants de ces transactions sont additionnés afin de produire le solde du début et de fin de période

- si la transaction est contenue dans la période, elle est imprimée.

\section{STRUCTURE DE DONNÉES INCLUSES}

numéro de l'écriture: 4 caractères alphanumériques

code de transaction: 1 caractère numérique (1)

date de l'écriture : 8 caractères alphanumériques

numéro de compte: 4 caractères numériques

numéro du sous-compte (succursale) s'il y a lieu: 2 caractères numériques

montant: maximum 8 caractères numériques dont 2 décimales

description: 60 caractères alphanumériques

1- $\operatorname{cod} \theta$ de transaction:

(1) transaction au joumal général

(2) transaction au joumal des rovenus

(3) transaction au joumal de la facturation

(4) transaction au caisse recettes

(5) transaction au caise déboursés

(6) transaction au joumal des acahts 
Flux de données

\section{Grand-livre cumulatif, 5.6.4-g}

REF. SOURCE: $\quad 5.6 .4 \quad$ DESCRIPTION: Produire grand-livre cumulatif

REF. DESTIN.: $\quad$ g DESCRIPTION: Directeur administratif

\section{DESCRIPTION DÉTAILLÉE}

Le grand-livre cumulatif contient toutes les transactions du mois ayant modifié la situation financière des entreprises du groupe Harel Drouin. Ces transactions sont regroupées par comptes et nous retrouvons deux catégories de comptes: les comptes d'opérations et les comptes de bilan.

STRUCTURE DE DONNÉES INCLUSES

pour chaque compte du grand-livre:

le solde d'ouverture

toutes les écritures de journal ayant affecté le compte

les solde de la fin

VOLUME

Harel Drouin, et associés: 1

Harel Drouin Gestion Conseil: 1

Société d'informatique de gestion: 1

Total par mois: 
Flux de données

\section{Nom des comptes, D12-5.6.4}

REF. SOURCE: D12 DESCRIPTION: Charte de comptes

REF. DESTIN.: $\quad 5.6 .4 \quad$ DESCRIPTION: $\quad$ Produire grand-livre cumulatif

DESCRIPTION DÉTAILLÉE

Contient le nom de comptes correspondant aux numéros des comptes qui ont été lus dans la charte de comptes. Le nom de chaque compte est imprimé à coté du numéro de compte. Le tout est suivis des transactions ayant affecté le compte durant le mois.

STRUCTURE DE DONNÉES INCLUSES

nom: $\mathbf{4 0}$ caractères alphanumériques

\section{VOLUME}

Harel Drouin, et associés: environ 200

Harel Drouin Gestion Conseil: $\quad$ environ 200

Société d'informatique de gestion: environ 200

Total par semaine: environ 600 
Flux de données

\section{Numéros de comptes, D12-5.6.4}

REF. SOURCE: D12 DESCRIPTION: Charte de comptes

REF. DESTIN.: $\quad 5.6 .4 \quad$ DESCRIPTION: Produire grand-livre cumulatif

DESCRIPTION DÉTAILÉE

Ce flux de données contient tous les numéros de comptes inscrit dans la charte de compte. Il est employé pour imprimer le grand-livre. Pour chaque compte inscrit dans la charte de compte, le logiciel calcule le solde au début du mois, imprime les transactions du mois et calcule le solde à la fin du mois.

STRUCTURE DE DONNÉES INCLUSES

numéro: 4 caractères numériques

VOLUME

Harel Drouin, et associés: environ 200

Harel Drouin Gestion Conseil: environ 200

Société d'informatique de gestion: environ 200

Total par semaine: environ 600 
Flux de données

\title{
Écritures de journal, D8-5.6.4
}

\author{
REF. SOURCE: D8 DESCRIPTION: Écritures de journal \\ REF. DESTIN:: $\quad$ 5.6.4 DESCRIPTION: $\quad$ Produire grand-livre cumulatif
}

\section{DESCRIPTION DÉTAILLÉE}

Ce flux contient l'ensemble des transactions ayant modifié la situation des entreprises du groupe Harel Drouin. Ces transactions sont lues et imprimées sur le rapport. Le montant de chaque transaction est additionné afin d'imprimer le solde du compte.

\section{STRUCTURE DE DONNÉES INCLUSES}

numéro de l'écriture: 4 caractères alphanumériques

code de transaction: 1 caractère numérique ${ }^{(1)}$

date de l'écriture : 8 caractères alphanumériques

numéro de compte: 4 caractères numériques

numéro du sous-compte (succursale) s'il y a lieu: 2 caractères numériques

montant: maximum 8 caractères numériques dont 2 décimales

description: 60 caractères alphanumériques

1- code de transaction: 
VOLUME

Harel Drouin, et associés: plusieurs milliers par années

Harel Drouin Gestion Conseil: $\quad$ plusieurs milliers par années

Société d'informatique de gestion: plusieurs milliers par années

Total par semaine: $\quad$ plusieurs milliers par années 
Flux de données

\section{Transactions, D7-5.6.5}

REF. SOURCE: D7 DESCRIPTION: Auxiliaire des travaux en cours

REF. DESTIN.: $\quad 5.6 .5 \quad$ DESCRIPTION: $\quad$ Produire auxiliaire des travaux en cours

\section{DESCRIPTION DÉTAILLÉE}

Contient toutes les transactions ayant affecté les comptes de clients. ces transactions peuvent être des transactions de temps, des déboursés, de la facturation et du profit et pertes.

\section{STRUCTURE DE DONNÉES INCLUSES}

\section{Dans le cas du temps:}

numéro de l'employé ou associé: 3 caractères numériques

nom de l'employé: 40 caractères alphanumériques

code d'activité correspondant au temps: 3 caractères numériques

date de la transaction: 8 caractères alphanumériques

code de transaction: 1 caractère numérique ${ }^{(1)}$

numéro de l'écriture de journal: 4 caractères alphanumériques

temps travaillé sur le dossier: $\quad 5$ caractères numériques dont deux décimales.

taux horaire de l'employé ou associé: 3 caractères numériques

coût de la transaction: 9 caractères numériques dont deux décimales

Dans le cas de déboursés facturables:

code d'activité correspondant au déboursé: 3 caractères numériques

1- code de transaction:

(1) transaction au journal général

(2) transaction au joumal des revenus

(3) transaction au joumal de la facturation

(4) transaction au caisse recettes

(5) transaction au caise déboursés

(6) transaction au joumal des achats 
date de la transaction: 8 caractères alphanumériques

code de transaction: 1 caractère numérique ${ }^{(1)}$

numéro de l'écriture de journal: 4 caractères alphanumériques

description du déboursé: 40 caractères numériques

coût du déboursé: 9 caractères numériques dont deux décimales

\footnotetext{
1- code de transaction: (1) transaction au joumal général

(2) transaction au joumal des revenus

(3) transaction au joumal de la facturation

(4) transaction au caisse recettes

(5) transaction au caise déboursés

(6) transaction au joumal des achats
} 
Dans le cas de facturation:

code d'activité correspondant à une facture: 3 caractères numériques

date de la facture: 8 caractères alphanumériques

code de transaction: 1 caractère numérique ${ }^{(1)}$

numéro de l'écriture de journal: 4 caractères alphanumériques

montant de la facture: 9 caractères numériques

description de la facture: 60 caractères alphanumériques

Dans le cas de transactions de profits et pertes:

code d'activité correspondant à une écriture de profits et pertes: 3 caractères numériques

date de la transaction: 8 caractères alphanumériques

code de transaction: 1 caractère numérique ${ }^{(2)}$

numéro de l'écriture de journal: 4 caractères alphanumériques

montant de l'écriture: 9 caractères numériques

description de l'écriture: 60 caractères alphanumériques

1- code de transaction: (1) transaction au joumal général

(2) transaction au joumal des revenus

(3) transaction au journal de la facturation

(4) transaction au caisse recettes

(5) transaction au caise déboursés

(6) transaction au joumal des achats

2- code de transaction:(1) transaction au joumal général

(2) transaction au joumal des revenus

(3) transaction au joumal de la facturation

(4) transaction au caisse recettes

(5) transaction au caise déboursés

(6) transaction au joumal des achats 
VOLUME

Harel Drouin, et Associés: $\quad 105,840^{(1)}+250^{(2)}+100^{(3)}+100^{(4)}$

Harel Drouin Gestion Conseil: $\quad 176,400+20+20+20$

Société d'informatique de gestion: $26.460+100+25+20$

Total par mois: $\quad 309,355$

1- nombre de transactions de feuilles de temps/semaines * 4

2- transactions de débourses par mois

3- factures par mois

4- transactions de profits et pertes par mois. Chaque facture produite engendre automatiquement la production d'une écritures de profits et pertes. 
Flux de données

\title{
Auxiliaire des travaux en cours, $5.6 .5-\mathrm{g}$
}

\author{
REF. SOURCE: $\quad$ 5.6.5 DESCRIPTION: Produire auxiliaire des travaux en cours \\ REF. DESTIN.: $\quad$ g $\quad$ DESCRIPTION: Directeur administratif
}

\section{DESCRIPTION DÉTAILÉE}

Contient toutes les transactions du mois ayant affecté les comptes de clients. Ces transactions concernent le temps travaillé dans le dossier du client, les déboursés engagés pour effectué le dossier du client, la facturation et les écritures de profits et pertes.

\section{STRUCTURE DE DONNÉES INCLUSES}

Pour chaque client inscrit dans le fichier maître client:

numéro du client: 8 caractères numériques

nom du client: 60 caractères alphanumériques

solde du début

temps: 9 caractères numériques dont deux décimales

déboursés: 9 caractères numériques dont deux décimales

facturation: 9 caractères numériques dont deux décimales

profits et pertes: 9 caractères numériques dont deux décimales

pour chaque transaction ayant affecté le compte du client:

Dans le cas du temps:

numéro de l'employé ou associé: 3 caractères numériques

nom de l'employé: $\mathbf{4 0}$ caractères alphanumériques

code d'activité correspondant au temps: 3 caractères numériques

date de la transaction: 8 caractères alphanumériques 
code de transaction: 1 caractère numérique ${ }^{(1)}$

numéro de l'écriture de journal: 4 caractères alphanumériques

temps travaillé sur le dossier: $\quad 5$ caractères numériques dont deux décimales.

taux horaire de l'employé ou associé: 3 caractères numériques

coût de la transaction: 9 caractères numériques dont deux décimales

Dans le cas de déboursés facturables:

code d'activité correspondant au déboursé: 3 caractères numériques

date de la transaction: 8 caractères alphanumériques

code de transaction: 1 caractère numérique (2)

numéro de l'écriture de journal: 4 caractères alphanumériques

description du déboursé: 40 caractères numériques

coût du déboursé: 9 caractères numériques dont deux décimales

1- code de transaction: (1) transaction au joumal général

(2) transaction au joumal des revenus

(3) transaction au joumal de la facturation

(4) transaction au caisse recettes

(5) transaction au caise déboursés

(6) transaction au joumal des achats

2- code de transaction: (1) transaction au journal général

(2) transaction au journal des revenus

(3) transaction au joumal do la facturation

(4) transaction au caisse recettes

(5) transaction au caise déboursés

(6) transaction au journal des achats 
Dans le cas de facturation:

code d'activité correspondant à une facture: 3 caractères numériques

date de la facture: 8 caractères alphanumériques

code de transaction: 1 caractère numérique (1)

numéro de l'écriture de journal: 4 caractères alphanumériques

montant de la facture: 9 caractères numériques

description de la facture: 60 caractères alphanumériques

Dans le cas de transactions de profits et pertes:

code d'activité correspondant à une écriture de profits et pertes: 3 caractères numériques

date de la transaction: 8 caractères alphanumériques

code de transaction: 1 caractère numérique ${ }^{(2)}$

numéro de l'écriture de journal: 4 caractères alphanumériques

montant de l'écriture: 9 caractères numériques

description de l'écriture: 60 caractères alphanumériques

1- code de transaction:(1) transaction au joumal général

(2) transaction au joumal des revenus

(3) transaction au journal de la facturation

(4) transaction au caisse recettes

(5) transaction au caise déboursés

(6) transaction au journal des achats

2- code de transaction:(1) transaction au joumal général

(2) transaction au joumal des revenus

(3) transaction au joumal de la facturation

(4) transaction au caisse recettes

(5) transaction au caise déboursés

(6) transaction au joumal des achats 
solde à la fin

temps: 9 caractères numériques dont deux décimales déboursés: 9 caractères numériques dont deux décimales facturation: 9 caractères numériques dont deux décimales profits et pertes: 9 caractères numériques dont deux décimales

VOLUME

Harel Drouin, et associés: $\quad 1$

Harel Drouin Gestion Conseil: 1

Société d'informatique de gestion: 1

Total par mois: $\quad 3$ 
Flux de données

\title{
Période, g-5.6.5
}

\author{
REF. SOURCE: $\mathrm{g}$ DESCRIPTION: Directeur administratif \\ REF. DESTIN.: $\quad 5.6 .5 \quad$ DESCRIPTION: $\quad$ Produire auxiliaire des travaux en cours
}

DESCRIPTION DÉTAILLÉE

Ce flux contient le mois pour la quelle on désire produire l'auxiliaire des travaux en cours.

STRUCTURE DE DONNÉES INCLUSES

mois: 2 caractères numériques

VOLUME

Harel Drouin, et associés: $\quad 12$

Harel Drouin Gestion Conseil: 12

Société d'informatique de gestion: 12

Total par semaine: $\quad 36$ 
Flux de données

\section{Numéros de clients, D2-5.6.5}

REF. SOURCE: D2 DESCRIPTION: Fichier maitre clients

REF. DESTIN.: $\quad 5.6 .5 \quad$ DESCRIPTION: Produire l'auxiliaire des travaux en cours

DESCRIPTION DÉTAILLÉE

Ce flux contient tous les numéros des clients qui sont inscrits dans le fichier maître clients. Ces numéros de clients sont employés lors de la production de l'auxiliaire des travaux en cours.

STRUCTURE DE DONNÉES INCLUSES

numéro du client: 8 caractères numériques

VOLUME

Harel Drouin, et associés: environ 3000

Harel Drouin Gestion Conseil: $\quad$ environ 250

Société d'informatique de gestion: environ 200

Total par semaine: environ $\mathbf{3 4 5 0}$ 
Flux de données

\section{Noms, D2-5.6.5}

REF. SOURCE: D2 DESCRIPTION: Fichier maître clients

REF. DESTIN.: $\quad 5.6 .5 \quad$ DESCRIPTION: Produire l'auxiliaire des travaux en cours

\section{DESCRIPTION DÉTAILLÉEE}

Contient les noms des clients qui sont inscrits dans le fichier maître clients. Ces noms sont employés lors de la production de l'auxiliaire des travaux en cours.

STRUCTURE DE DONNÉES INCLUSES

nom du client: 60 caractères alphanumériques

VOLUME

Harel Drouin, et associés: environ $\mathbf{3 0 0 0}$

Harel Drouin Gestion Conseil: environ 250

Société d'informatique de gestion: environ $\mathbf{2 0 0}$

Total par semaine: environ $\mathbf{3 4 5 0}$ 
Flux de données

\section{Transactions, D11-5.6.6}

REF. SOURCE: D11 DESCRIPTION: Auxiliaire des comptes à recevoir

REF. DESTIN.: $\quad$ 5.6.6 DESCRIPTION: $\quad$ Produire l'auxiliaire des C.A.R.

\section{DESCRIPTION DÉTAILLÉE}

Contient toutes les transactions ayant affecté l'auxiliaire des comptes à recevoir. Ces transactions comprennent des transactions de facturation et des transactions d'encaissement.

\section{STRUCTURE DE DONNÉES INCLUSES}

Pour chaque client inscrit dans le fichier maître clients:

Pour chaque facture:

code de transaction: 1 caractère numérique

numéro de l'écriture: 4 caractères numériques

date de la transaction: 8 caractères alphanumériques

numéro du client: 5 caractères numériques

numéro du projet: 3 caractères numériques

numéro de référence: 7 caractères numériques

description de la facture: 60 caractères alphanumériques

montant: 7 caractères numériques dont deux décimales

pour chaque chèque:

code de transaction: 1 caractère numérique

numéro de l'écriture: 4 caractères numériques

numéro du client: 5 caractères numériques

numéro du projet: 3 caractères numériques 
montant du chèque: 7 caractères numériques

numéro de référence de la facture: 7 caractères numériques

\section{VOLUME}

Harel Drouin, et associés: environ 3000

Harel Drouin Gestion Conseil: environ 240

Société d'informatique de geson: environ 1200

Total par mois: environ 4400 
Flux de données

Numéros de clients, D2-5.6.6

REF. SOURCE: D2 DESCRIPTION: Fichier maître clients

REF. DESTIN.: $\quad$ 5.6.6 DESCRIPTION: $\quad$ Produire l'auxiliaire des C.A.R.

DESCRIPTION DÉTAILLÉE

Ce flux contient tous les numéros des clients qui sont inscrits dans le fichier maître clients. Ces numéros de clients sont employés lors de la production de l'auxiliaire des comptes à recevoir.

STRUCTURE DE DONNÉES INCLUSES

numéro du client: 8 caractères numériques

VOLUME

Harel Drouin, et associés: environ 3000

Harel Drouin Gestion Conseil: $\quad$ environ 250

Société d'informatique de geson: environ 200

Total par semaine: $\quad$ environ 3450 
Flux de données

\section{Noms, D2-5.6.5}

REF. SOURCE: D2 DESCRIPTION: Fichier maître clients

REF. DESTIN.: $\quad 5.6 .5 \quad$ DESCRIPTION: Produire l'auxiliaire des comptes à recevoir

\section{DESCRIPTION DÉTAILÉE}

Contient les noms des clients qui sont inscrits dans le fichier maître clients. Ces noms sont employés lors de la production de l'auxiliaire des travaux en cours.

STRUCTURE DE DONNÉES INCLUSES

nom du client: 60 caractères alphanumériques

VOLUME

Harel Drouin, et associés: environ $\mathbf{3 0 0 0}$

Harel Drouin Gestion Conseil: environ 250

Société d'informatique de gestion: environ 200

Total par semaine: environ $\mathbf{3 4 5 0}$ 
Flux de données

\title{
Auxiliaire des comptes a recevoir, $5.6 .5-\mathrm{g}$
}

REF. SOURCE: $\quad 5.6 .5$

\author{
DESCRIPTION: Produire l'auxiliaire des comptes à \\ recevoir
}

REF. DESTIN.: $\quad g \quad$ DESCRIPTION: Directeur administratif

DESCRIPTION DÉTAILLÉE

Document qui présente toutes les factures non-payées, et ce, par client. Dans ce document, on retrouve également le montant à recevoir par client et le montant total des comptes à recevoir.

STRUCTURE DE DONNÉES INCLUSES

numéro du client: 8 caractères numériques

nom du client: 60 caractères alphanumériques

pour chaque facture:

code de transaction: 1 caractère numérique

numéro de l'écriture: 4 caractères numériques

date de la transaction: 8 caractères alphanumériques

numéro du client: 5 caractères numériques

numéro du projet: 3 caractères numériques

numéro de référence: 7 caractères numériques

description de la facture: 60 caractères alphanumériques

montant: 7 caractères numériques dont deux décimales 
pour chaque recette qui ne couvre pas entièrement une facture ou qui n'a pas de numéro de référence:

code de transaction: 1 caractère numérique

numéro de l'écriture: 4 caractères numériques

numéro du client: 5 caractères numériques

numéro du projet: 3 caractères numériques

montant du chèque: 7 caractères numériques

numéro de référence de la facture ( $s^{\prime} i$ y a lieu): 7 caractères numériques

\section{VOLUME}

Harel Drouin, et associés: environ 250

Harel Drouin Gestion Conseil: environ 20

Société d'informatique de gestion: environ 100

Total par mois: $\quad$ environ 370 
Flux de données

\section{Transactions, D6-5.6.7}

REF. SOURCE: D6 DESCRIPTION: Auxiliaire des comptes à payer

REF. DESTIN.: $\quad 5.6 .7 \quad$ DESCRIPTION: $\quad$ Produire l'auxiliaire des comptes à payer

\section{DESCRIPTION DÉTAILLÉE}

Contient toutes les transactions ayant affecté les comptes fournisseurs. Ces transactions peuvent comprendre des factures, des paiements ou des avances.

STRUCTURE DE DONNÉES INCLUSES

Dans le cas de factures:

numéro du fournisseur: 4 caractères numériques

numéro de l'écriture: 4 caractères numériques

code de transaction: 1 caractère numérique

date de la facture: 8 caractères alphanumériques

montant de la facture: $\quad$ maximum 8 caractères numériques dont 2 décimales

description des articles commandés: généralement le numéro de la facture

Dans le cas de comptes de dépenses:

date: caractères alphanumériques

numéro de l'employé: 3 caractères numériques

montant: 7 caractères numériques dont deux décimales 


\section{VOLUME}

Harel Drouin, et Associés

Harel Drouin Gestion Conseil

Société d'informatique de gestion

Total par mois
$: 700^{(1)}+200^{(2)}$

$: 50+40$

$: 50+50$

$: 800+290=545$

1- pour les factures et les chèques payant ces factures.

2- pour les comptes de dépenses et les chèques payant ces dépenses. 
Flux de données

\section{Période, g-5.6.7}

REF. SOURCE: $\quad \mathrm{g} \quad$ DESCRIPTION: Directeur administratif

REF. DESTIN.: $\quad$ 5.6.7 DESCRIPTION: Produire l'auxiliaire des comptes à payer

DESCRIPTION DÉTAILLÉE

Contient le mois pour lequel nous désirons imprimer l'auxiliaire des comptes à payer.

STRUCTURE DE DONNÉES INCLUSES

mois: 2 caractères numériques

VOLUME

Harel Drouin, et associés: $\quad 12$

Harel Drouin Gestion Conseil: 12

Société d'informatique de gestion: 12

Total par semaine: $\quad 36$ 
Flux de données

\section{Auxiliaire des comptes à payer, 5.6.7-g}

REF. SOURCE: $\quad$ 5.6.7 DESCRIPTION: Produire l'auxiliaire des comptes à payer REF. DESTIN.: $\quad$ g DESCRIPTION: Directeur administratif

\section{DESCRIPTION DÉTAILLEEE}

Contient toutes l'information relative aux comptes à payer. Dans l'auxiliaire des comptes à payer, nous retrouvons l'ensemble des factures impayées et les factures pour lesquelles un paiement partiel a été effectué. Cette auxiliaire nous fournit, pour chaque fournisseur, de l'information sur les soldes à payer.

STRUCTURE DE DONNÉES INCLUSES

numéro du fournisseur: 4 caractères numériques

nom du fournisseur: 60 caractères alphanumériques

Dans le cas de factures:

numéro de l'écriture: 4 caractères numériques

code de transaction: 1 caractère numérique

date de la facture: 8 caractères alphanumériques

montant de la facture: $\quad$ maximum 8 caractères numériques dont 2 décimales

description: le numéro de la facture

Dans le cas de comptes de dépenses:

numéro de l'écriture: 4 caractères numériques

code de transaction: 1 caractère numérique

date: caractères alphanumériques

montant: 7 caractères numériques dont deux décimales

description: généralement dépenses facturables 
Dans les cas de paiements partiels:

numéro de l'écriture: 4 caractères numériques

code de transaction: 1 caractère numérique

date du paiement: 8 caractères alphanumériques

montant du paiement: maximum 8 caractères numériques dont 2 décimales

description: le numéro de la facture

\section{VOLUME}

Harel Drouin, et Associés

$: 700^{(1)}+200^{(2)}$

Harel Drouin Gestion Conseil

$: 50+40$

Société d'informatique de gestion

$: 50+50$

Total par mois

$: 800+290=545$

1- pour les factures et les chèques payant ces factures.

2- pour les comptes de dépenses et les chèques payant ces dépenses. 
Flux de données

\title{
Écritures de journal, D8-5.6.8
}

\author{
REF. SOURCE: D8 DESCRIPTION: Écritures de journal \\ REF. DESTIN.: $\quad 5.6 .8 \quad$ DESCRIPTION: Produire les états financiers
}

\section{DESCRIPTION DÉTAILLÉE}

Contient toutes les transactions ayant affecté les comptes du grand-livre. ces transaction sont lues et leur montant compilé afin de produire les états financiers. Les soldes de certains compte de grand-livre sont regroupés afin de déterminer les soldes des postes au bilan et à l'états des résultats.

STRUCTURE DE DONNÉES INCLUSES

numéro de compte: 4 caractères numériques

numéro du sous-compte (succursale) s'il y a lieu: 2 caractères numériques

montant: maximum 8 caractères numériques dont 2 décimales

description: 60 caractères alphanumériques

VOLUME

Harel Drouin, et associés: plusieurs milliers par année

Harel Drouin Gestion Conseil: $\quad$ plusieurs milliers par année

Société d'informatique de gestion: plusieurs milliers par année

Total par année:

plusieurs milliers par année 
Flux de données

\section{Information sur les comptes, D12-5.6.8}

REF. SOURCE: D12 DESCRIPTION: Charte de comptes

REF. DESTIN.: $\quad 5.6 .8 \quad$ DESCRIPTION: Produire les états financiers

DESCRIPTION DÉTAILLÉE

Contient de l'information nous indiquant quels comptes regrouper ensemble afin de produire les états financiers. Cela nous informe également sur les comptes "titre" contenu dans l'état financier.

STRUCTURE DE DONNÉES INCLUSES

numéro du compte: 4 caractères numériques

nom du compte: 40 caractères alphanumériques

type de compte: détail

entête

regroupement

numéros de comptes inclus dans le regroupement ${ }^{(1)}$ : un dizaine de numéro de compte qui se lisent par multiple de quatre.

VOLUME

Harel Drouin, et associés: environ 220

Harel Drouin Gestion Conseil: $\quad$ environ 220

Société d'informatique de gestion: environ 220

Total par mois: environ 660 
Flux de données

\section{États financiers, 5.6.8-g}

REF. SOURCE: $\quad 5.6 .8 \quad$ DESCRIPTION: Produire les états financiers

REF. DESTIN: $\quad g \quad$ DESCRIPTION: Directeur administratif

DESCRIPTION DÉTAILLÉE

Contient des rapports représentant la situation des entreprises du groupe Harel Drouin. Ces rapports comprennent un état des résultats et un bilan.

STRUCTURE DE DONNÉES INCLUSES

Pour l'états des résultats:

Comprend tous les postes d'opération, c'est à dire les postes de revenus et dépenses.

Pour le bilan:

Comprend tous les postes d'actif, de passif et de l'avoir des sociétaires ou actionnaires selon le cas.

VOLUME

Harel Drouin, et associés: 1

Harel Drouin Gestion Conseil: 1

Société d'informatique de gestion: 1

Total par mois: $\quad 4$ 


\section{Index}

\section{Processus 2.0, Compiler feuille de temps 1}

Diagramme de flux d'information $\ldots \ldots \ldots \ldots \ldots \ldots \ldots, 2$

2.0 Compiler feuille de temps $\ldots \ldots \ldots \ldots \ldots \ldots \ldots \ldots \ldots \ldots \ldots \ldots \ldots \ldots \ldots \ldots \ldots \ldots \ldots$

Processus de traitement $\ldots \ldots \ldots \ldots \ldots \ldots \ldots \ldots \ldots \ldots \ldots, 4$

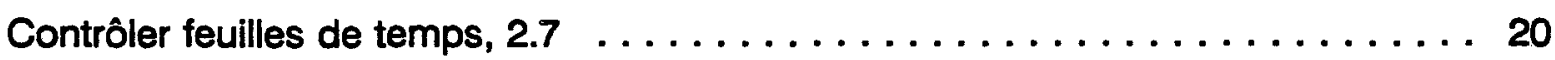

Corriger les transactions, $2.3 \ldots \ldots \ldots \ldots \ldots \ldots \ldots \ldots \ldots \ldots \ldots \ldots$

Créer un nouveau client, $2.4 \ldots \ldots \ldots \ldots \ldots \ldots \ldots \ldots \ldots \ldots \ldots \ldots \ldots$

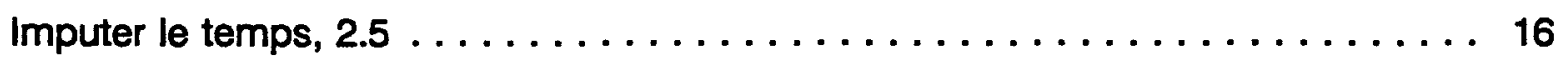

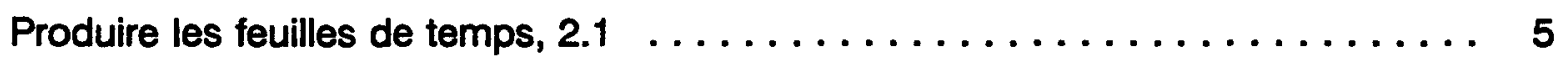

Produire rapport statistique, $2.6 \ldots \ldots \ldots \ldots \ldots \ldots \ldots$

Saisir données feuilles de temps, $2.8 \ldots \ldots \ldots \ldots \ldots \ldots \ldots$

Valider feuilles de temps, $2.2 \ldots \ldots \ldots \ldots \ldots \ldots \ldots \ldots \ldots \ldots$

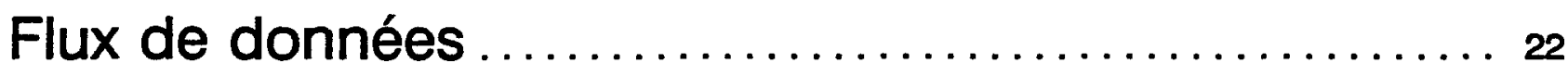

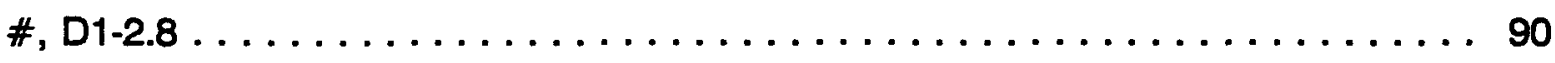

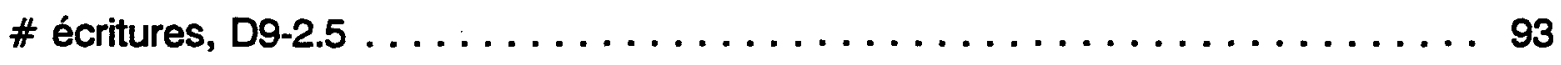

Code, $2.3-122 / 2 \ldots \ldots \ldots \ldots \ldots \ldots \ldots \ldots \ldots \ldots \ldots \ldots \ldots \ldots \ldots \ldots \ldots \ldots$

Code, $\mathrm{D} 2 / 2-2.8 \ldots \ldots \ldots \ldots \ldots \ldots \ldots \ldots \ldots \ldots \ldots \ldots \ldots \ldots \ldots \ldots \ldots$ 


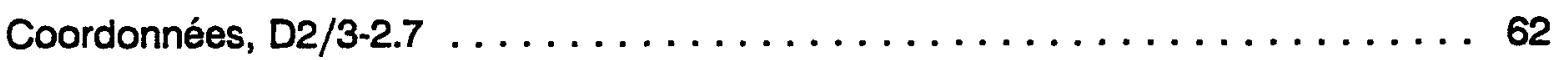

Corrections, a. $-2.3 \ldots \ldots \ldots \ldots \ldots \ldots \ldots \ldots \ldots \ldots \ldots \ldots \ldots \ldots$

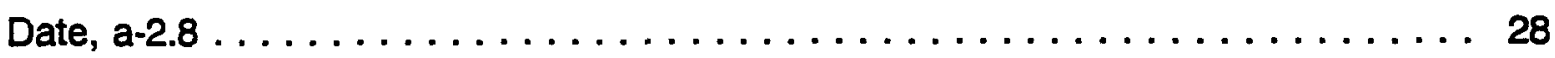

Date de la feuille de temps, A-2.7 $\ldots \ldots \ldots \ldots \ldots \ldots \ldots \ldots \ldots \ldots 69$

Date et numéro de l'associé, $2.5-\mathrm{D} 2 / 7 \ldots \ldots \ldots \ldots \ldots \ldots \ldots \ldots \ldots \ldots \ldots \ldots \ldots \ldots$

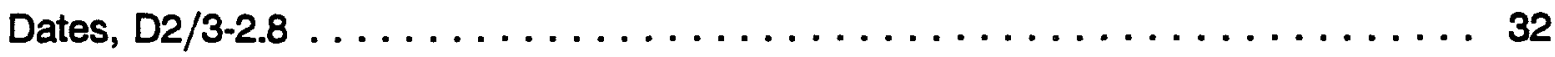

Données de feuilles de temps, $b-2.1 \ldots \ldots \ldots \ldots \ldots \ldots \ldots \ldots \ldots \ldots \ldots$

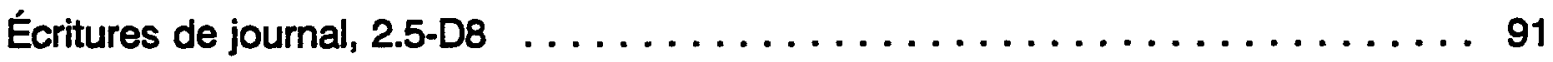

Feuilles complétées, $2.7-\mathrm{D} 2 / 3 \ldots \ldots \ldots \ldots \ldots \ldots \ldots \ldots \ldots \ldots \ldots$

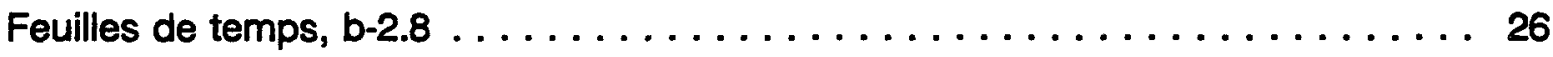

Feuilles de temps manquantes, $2.7-A \ldots \ldots \ldots \ldots \ldots \ldots \ldots \ldots \ldots 67$

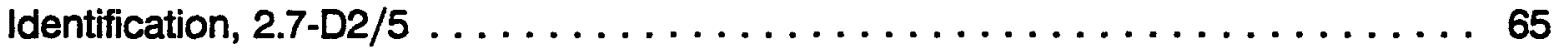

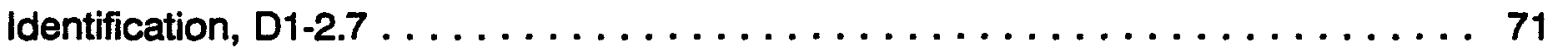

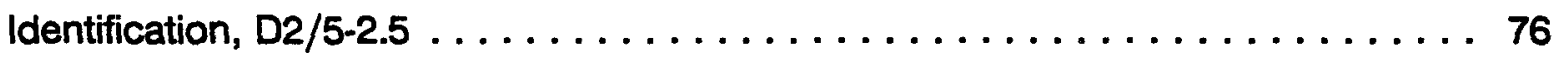

Information client, $2.3-2.4 \ldots \ldots \ldots \ldots \ldots \ldots \ldots \ldots \ldots \ldots \ldots \ldots .48$

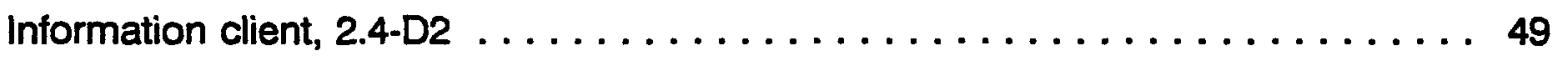

Information client, a.-2.4 $\ldots \ldots \ldots \ldots \ldots \ldots \ldots \ldots \ldots \ldots \ldots \ldots$

Informations pour rapports, $\mathrm{C}-2.6 \ldots \ldots \ldots \ldots \ldots \ldots \ldots \ldots \ldots \ldots \ldots \ldots \ldots \ldots$

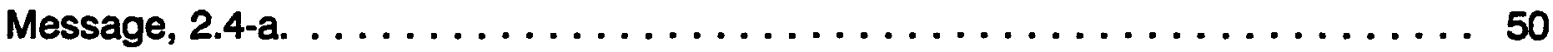

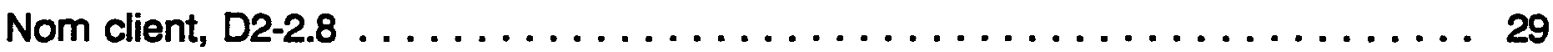

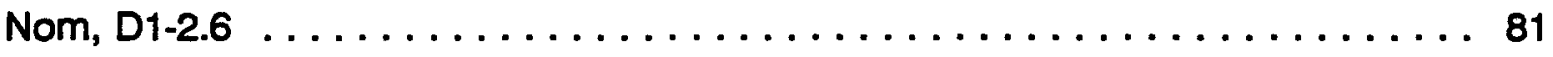

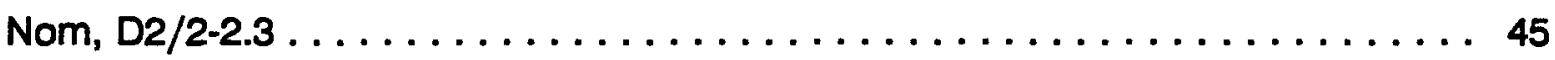

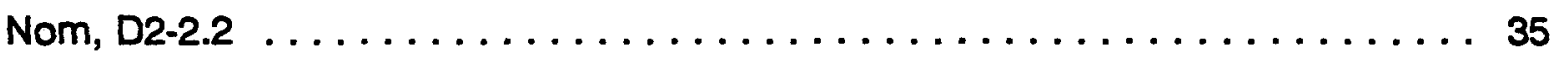

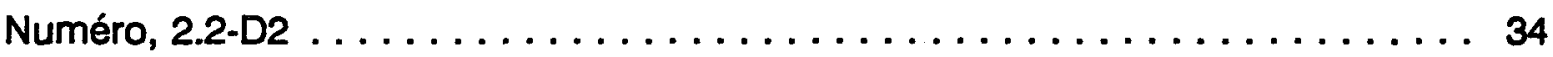

Numéro de l'associé, a.-2.7 $\ldots \ldots \ldots \ldots \ldots \ldots \ldots \ldots \ldots \ldots \ldots \ldots$

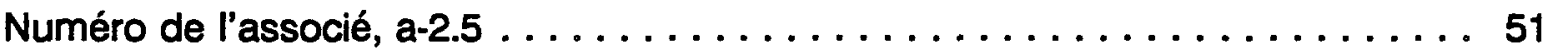

Numéro de l'associe, $D 1-2.5 \ldots \ldots \ldots \ldots \ldots \ldots \ldots \ldots \ldots \ldots \ldots \ldots$ 
Rapport de validation, $\mathrm{D} 2 / 1-2.5 \ldots \ldots \ldots \ldots \ldots \ldots \ldots \ldots \ldots$

Rapport de validation, $\mathrm{D} 2 / 1-\mathrm{a} . \ldots \ldots \ldots \ldots \ldots \ldots \ldots \ldots \ldots \ldots$

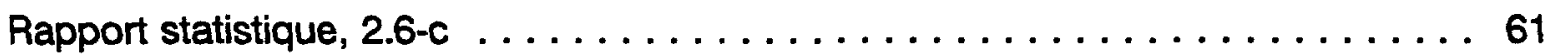

Taux horaire, $D 1-2.5 \ldots \ldots \ldots \ldots \ldots \ldots \ldots$

Temps supplémentaire, $2.7-\mathrm{D} 2 / 4 \ldots \ldots \ldots \ldots \ldots \ldots \ldots \ldots$

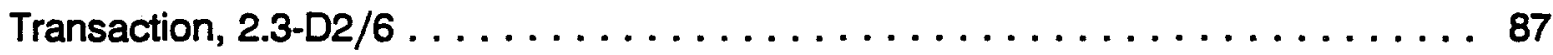

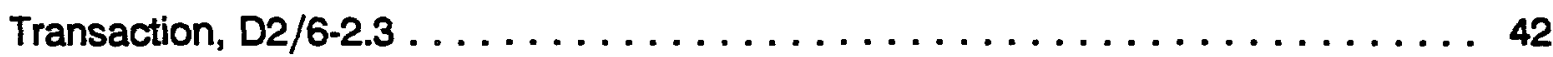

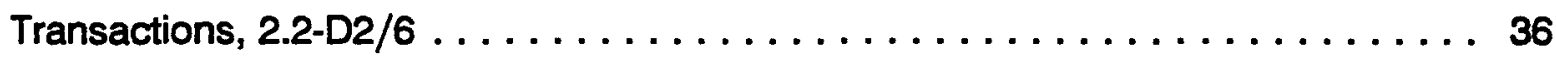

Transactions, $2.5-\mathrm{D} 2 / 6 \ldots \ldots \ldots \ldots \ldots \ldots \ldots \ldots \ldots$

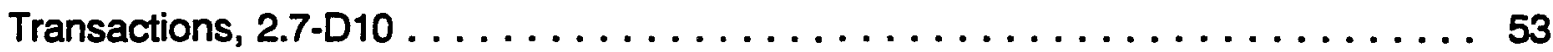

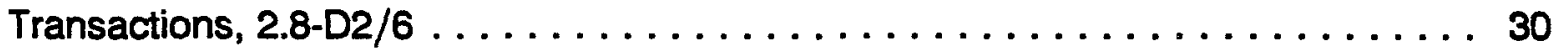

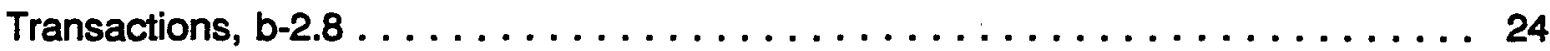

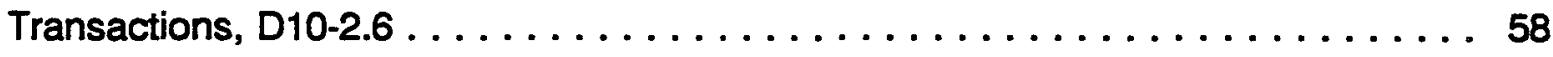

Transactions, $\mathrm{D} 2 / 6-2.2 \ldots \ldots \ldots \ldots \ldots \ldots \ldots \ldots \ldots$

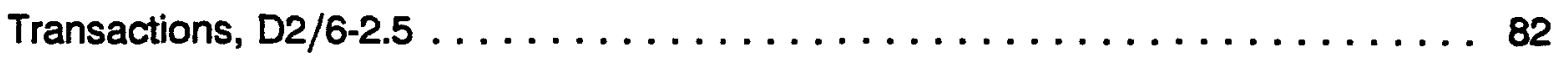

Transactions, $\mathrm{D} 2 / 6-2.7 \ldots \ldots \ldots \ldots \ldots \ldots \ldots \ldots \ldots$

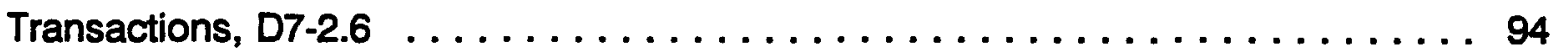

Transactions erronées, $2.2-\mathrm{D} 2 / 1 \ldots \ldots \ldots \ldots \ldots \ldots \ldots \ldots$

Transactions facturables, $2.5-D 7 \ldots \ldots \ldots \ldots \ldots \ldots \ldots$

\section{Processus 2.1, Produire les feuilles de}

temps 
2.1 Produire les feuille de temps $\ldots \ldots \ldots \ldots \ldots \ldots \ldots \ldots \ldots \ldots \ldots .98$

Processus de traitement $\ldots \ldots \ldots \ldots \ldots \ldots \ldots \ldots \ldots \ldots, 99$

Approuver feuilles de temps, $2.1 .4 \ldots \ldots \ldots \ldots \ldots \ldots \ldots \ldots \ldots \ldots$

Corriger feuille de temps, $2.1 .3 \ldots \ldots \ldots \ldots \ldots \ldots \ldots \ldots \ldots \ldots \ldots$

Enregistrer données feuilles-temps, $2.1 .1 \ldots \ldots \ldots \ldots \ldots \ldots \ldots \ldots$

Imprimer feuille de temps, $2.1 .2 \ldots \ldots \ldots \ldots \ldots \ldots \ldots \ldots \ldots \ldots \ldots$

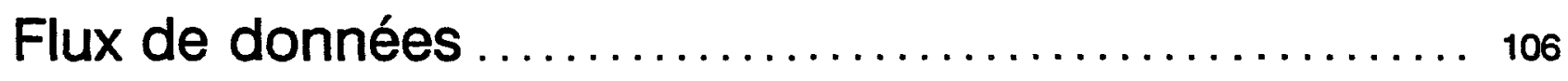

\# ass, 2.1.1-D2.1/2 ........................ 148

\# ass, $02.1 / 2-2.1 .3 \ldots \ldots \ldots \ldots \ldots \ldots \ldots \ldots \ldots \ldots \ldots \ldots \ldots .149$

Code, $2.1 .1-D 2.1 / 5 \ldots \ldots \ldots \ldots \ldots \ldots \ldots \ldots \ldots \ldots \ldots \ldots \ldots \ldots \ldots$

Code, $2.1 .3-\mathrm{D} 2.1 / 5 \ldots \ldots \ldots \ldots \ldots \ldots \ldots \ldots \ldots \ldots \ldots \ldots \ldots \ldots \ldots$

Coordonnées de la feuille de temps, $\mathrm{D} 2.1 / 3-2.1 .1 \ldots \ldots \ldots \ldots \ldots \ldots$

Coordonnées de la feuille de temps, $D 2.1 / 3-2.1 .2 \ldots \ldots \ldots \ldots \ldots \ldots \ldots$

Corrections, $2.1 .4-2.1 .3 \ldots \ldots \ldots \ldots \ldots \ldots \ldots \ldots \ldots \ldots \ldots \ldots .145$

Date de la feuille de temps, $2.1 .1-\mathrm{D} 2.1 / 3 \ldots \ldots \ldots \ldots \ldots \ldots \ldots \ldots \ldots$

Date de la feuille de temps, $2.1 .2-D 2.1 / 3 \ldots \ldots \ldots \ldots \ldots \ldots \ldots \ldots \ldots . \ldots 127$

Date de la feuille de temps, b-2.1.2 . . . . . . . . . . . . . . 124

Date feuille de temps, $b-2.1 .1 \ldots \ldots \ldots \ldots \ldots \ldots \ldots \ldots \ldots \ldots \ldots$

Données feuille de temps, $2.1 .1-D 2.1 / 6 \ldots \ldots \ldots \ldots \ldots \ldots \ldots \ldots \ldots \ldots$

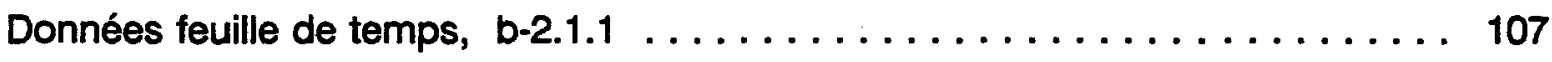

Feuille de temps, $2.1 .2-2.1 .3 \ldots \ldots \ldots \ldots \ldots \ldots \ldots \ldots \ldots \ldots \ldots \ldots$

Feuille de temps, $2.1 .2-2.1 .4 \ldots \ldots \ldots \ldots \ldots \ldots \ldots \ldots \ldots \ldots \ldots \ldots$

Feuilles de temps, $2.1 .4-\mathrm{D} 2.1 / 4 \ldots \ldots \ldots \ldots \ldots \ldots \ldots \ldots \ldots \ldots \ldots$

Identification, $2.1 .1-\mathrm{D} 2.1 / 2 \ldots \ldots \ldots \ldots \ldots \ldots \ldots \ldots \ldots \ldots \ldots \ldots \ldots \ldots$ 


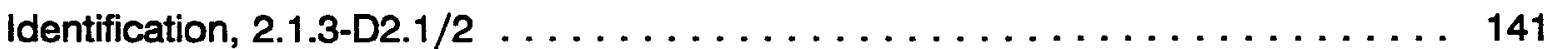

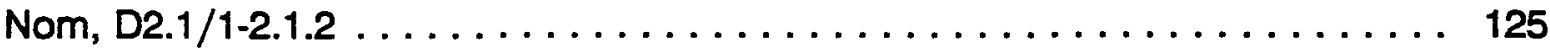

Nom, $02.1 / 2-2.1 .1 \ldots \ldots \ldots \ldots \ldots \ldots \ldots \ldots \ldots \ldots \ldots \ldots \ldots \ldots \ldots$

Nom de l'activité, $\mathrm{D} 2.1 / 5-2.1 .1 \ldots \ldots \ldots \ldots \ldots \ldots \ldots \ldots \ldots \ldots$

Nom de l'activité, $D 2.1 / 5-2.1 .3 \ldots \ldots \ldots \ldots \ldots \ldots \ldots \ldots \ldots$

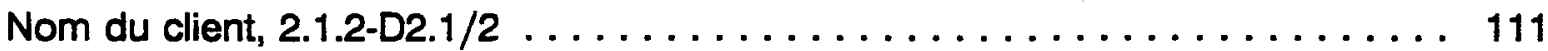

Nom du client, D2.1/2-2.1.3 . . . . . . . . . . . . . . . . . . . . . 140

Nom et numéro, $2.1 .1-D 2.2 / 1 \ldots \ldots \ldots \ldots \ldots \ldots \ldots \ldots$

Nom et numéro, b-2.1.1 . . . . . . . . . . . . . . . . . . . 109

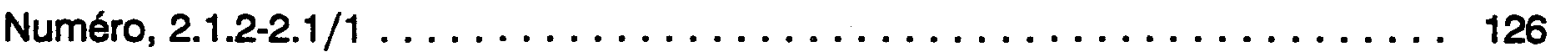

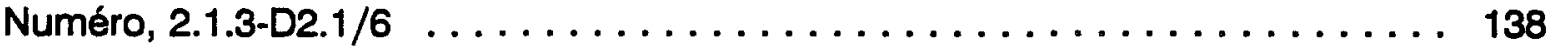

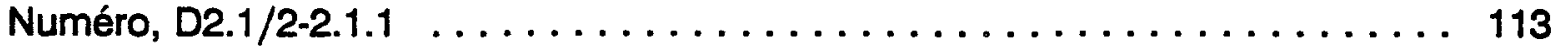

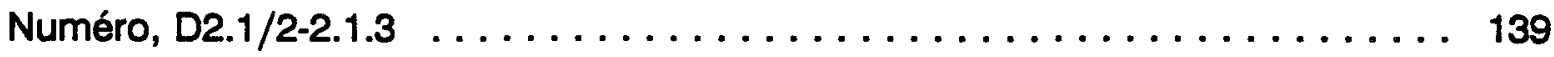

Numéro de la transaction, b-2.1.3 . . . . . . . . . . . . . . . . . 142

Transaction, $2.1 .3-D 2.1 / 6 \ldots \ldots \ldots \ldots \ldots \ldots \ldots \ldots \ldots \ldots$

Transactions, D2.1/6-2.1.2 . . . . . . . . . . . . . . . . . 134

\section{Processus 2.6, Produire rapports statisti-}

ques

\section{Processus 2.7, Contrôler feuilles de}


Diagramme de flux de données $\ldots \ldots \ldots \ldots \ldots \ldots \ldots, 166$

2.7 Contrôler feuilles de temps $\ldots \ldots \ldots \ldots \ldots \ldots \ldots \ldots \ldots \ldots \ldots \ldots .67$

Processus de traitement $\ldots \ldots \ldots \ldots \ldots \ldots \ldots \ldots \ldots \ldots, 168$

Calculer temps supplémentaire, $2.7 .3 \ldots \ldots \ldots \ldots \ldots \ldots \ldots \ldots \ldots \ldots 172$

Déterminer feuilles de temps manquantes, $2.7 .1 \ldots \ldots \ldots \ldots \ldots \ldots \ldots .6169$

Vérifier feuilles de temps manquantes, $2.7 .2 \ldots \ldots \ldots \ldots \ldots \ldots \ldots \ldots 171$

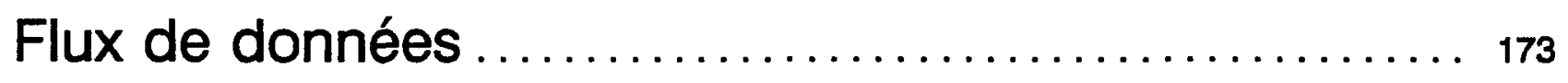

Coordonnées, $D 2 / 3-2.7 .1 \ldots \ldots \ldots \ldots \ldots \ldots \ldots \ldots \ldots \ldots \ldots \ldots \ldots \ldots$

Coordonnées, D2/3-2.7.3 ...................... 186

Date de la feuille de temps, a-2.7.2 $\ldots \ldots \ldots \ldots \ldots \ldots \ldots \ldots \ldots \ldots . \ldots \ldots$

Feuilles complétées, $2.7 .1-D 2 / 3 \ldots \ldots \ldots \ldots \ldots \ldots \ldots \ldots \ldots \ldots \ldots 179$

Feuilles de temps manquantes, $2.7 .2-a \ldots \ldots \ldots \ldots \ldots \ldots \ldots \ldots \ldots .176$

Identification, $2.7 .1-\mathrm{D} 2 / 5 \ldots \ldots \ldots \ldots \ldots \ldots \ldots \ldots \ldots \ldots \ldots \ldots$

Identification, D1-2.7.1 .......................... 182

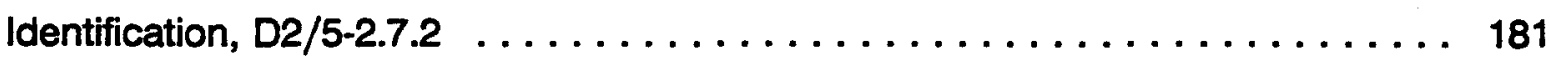

Numéro d'associé, a-2.7.2 $\ldots \ldots \ldots \ldots \ldots \ldots \ldots \ldots \ldots \ldots \ldots \ldots \ldots$

Temps supplémentaire, $2.7 .3-D 10 \ldots \ldots \ldots \ldots \ldots \ldots \ldots \ldots \ldots \ldots \ldots$

Transactions, $\mathrm{D} 2 / 6-2.7 .1 \ldots \ldots \ldots \ldots \ldots \ldots \ldots \ldots \ldots \ldots \ldots \ldots \ldots \ldots \ldots$

Transactions, $\mathrm{D} 2 / 6-2.7 .3 \ldots \ldots \ldots \ldots \ldots \ldots \ldots \ldots \ldots \ldots \ldots \ldots \ldots \ldots$ 


\section{Processus 5.2, Facturer les clients}

Diagramme de flux de données

190

5.2 Facturer les clients

Processus de traitement

Dactylographier la facture, $5.2 .2 \ldots \ldots \ldots \ldots \ldots \ldots$. . . . . . . . . . . 194

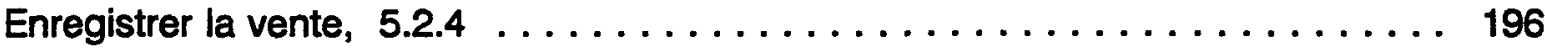

Enregistrer les recettes, $5.2 .6 \ldots \ldots \ldots \ldots \ldots \ldots \ldots$

Produire les factures, $5.2 .1 \ldots \ldots \ldots \ldots \ldots \ldots \ldots \ldots$

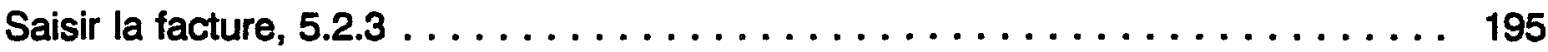

Saisir les chèques, $5.2 .5 \ldots \ldots \ldots \ldots \ldots \ldots \ldots$

Flux de données ............................... 200

Charges imputées, $D 7-5.2 .1 \ldots \ldots \ldots \ldots \ldots \ldots \ldots$

Chèques, g-5.2.5 ........................... 223

Copie de la facture, $5.2 .2-D 5.2 / 1 \ldots \ldots \ldots \ldots$. . . . . . . . . . . . . 209

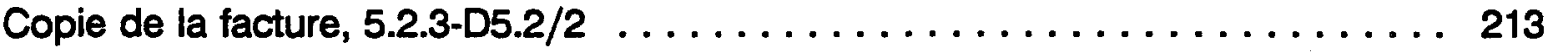

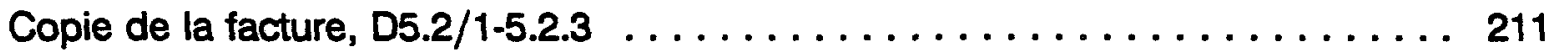

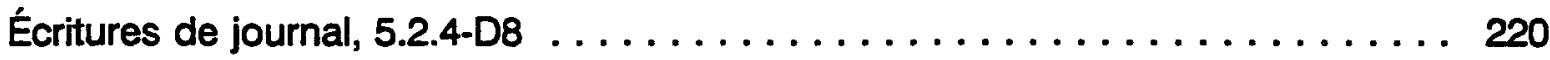

Écritures de journal, $5.2 .6-D 8 \ldots \ldots \ldots \ldots \ldots \ldots \ldots \ldots \ldots$

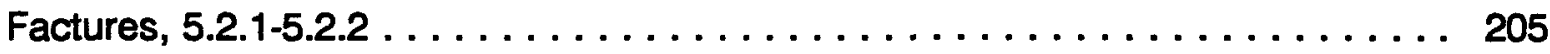

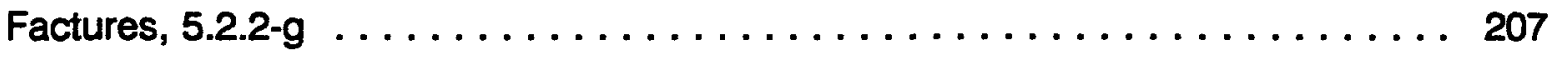

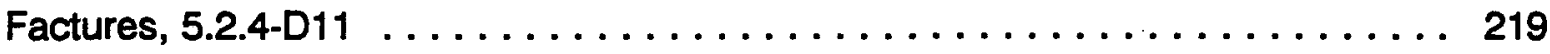


Factures, 5.2.4-D7 ........................... 217

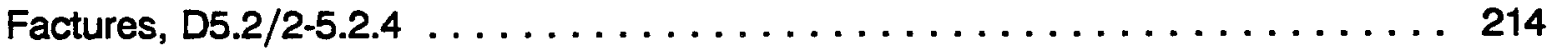

Information sur paiement, 5.2.6-D11 . . . . . . . . . . . . . . 229

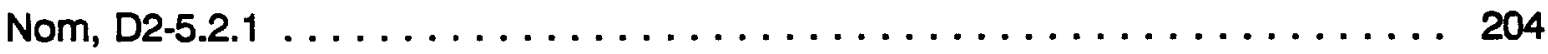

Numéro d'écriture, D9-5.2.4 ....................... 215

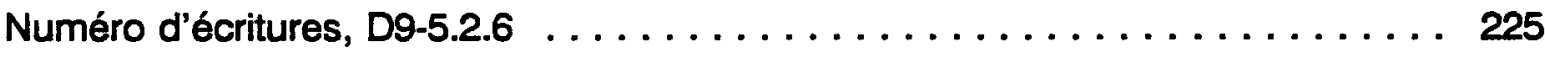

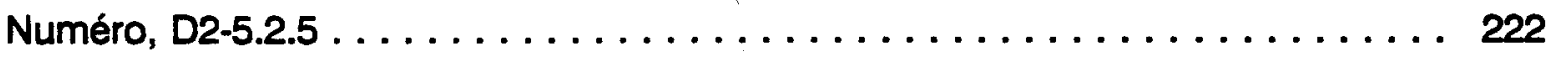

Numéro de facture, D11-5.2.5 ..................... 224

Recettes, $5.2 .5-D 5.2 / 4 \ldots \ldots \ldots \ldots \ldots \ldots \ldots \ldots \ldots \ldots \ldots \ldots \ldots .227$

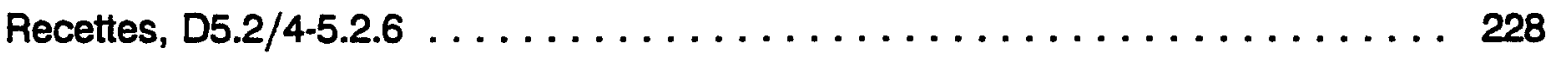

\section{Processus 5.5, Payer les fournisseurs .... ${ }_{232}$}

Diagramme de flux de données ................... 233

5.5 Payer les fournisseurs $\ldots \ldots \ldots \ldots \ldots \ldots \ldots \ldots \ldots \ldots \ldots \ldots \ldots \ldots$

Processus de traitement $\ldots \ldots \ldots \ldots \ldots \ldots \ldots \ldots \ldots \ldots \ldots \ldots \ldots$

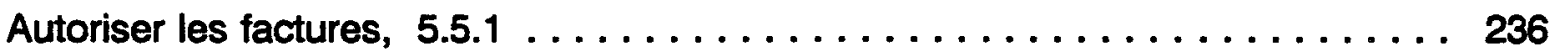

Enregistrer les nouveaux fournisseurs, $5.5 .8 \ldots \ldots \ldots \ldots \ldots \ldots \ldots \ldots 247$

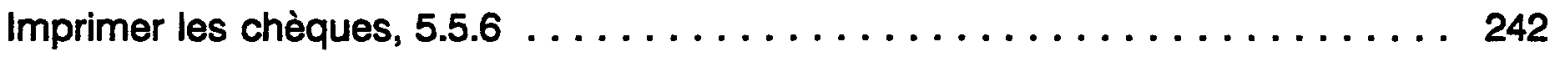

Imprimer liste des factures à payer, $5.5 .4 \ldots \ldots \ldots \ldots \ldots \ldots \ldots \ldots \ldots$

Imputer les dépenses, $5.5 .7 \ldots \ldots \ldots \ldots \ldots \ldots \ldots \ldots \ldots \ldots \ldots \ldots \ldots$

Inscrire les codes d'imputation, $5.5 .2 \ldots \ldots \ldots \ldots \ldots \ldots \ldots \ldots \ldots \ldots . \ldots \ldots$ 


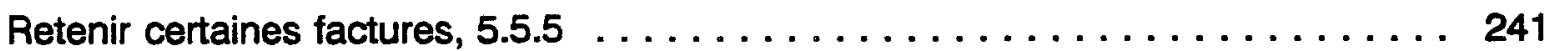

Saisir les factures, $5.5 .3 \ldots \ldots \ldots \ldots \ldots \ldots \ldots \ldots \ldots \ldots$

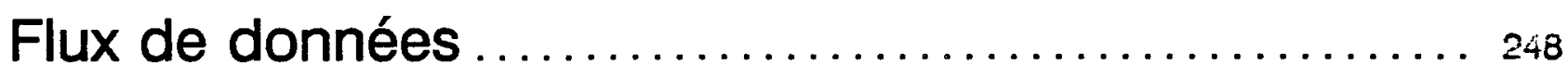

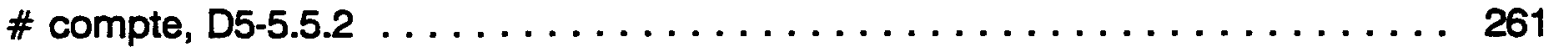

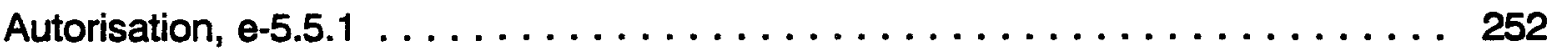

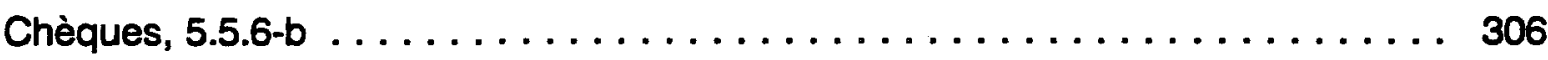

Chèques, $5.5 .6-d \ldots \ldots \ldots \ldots \ldots \ldots \ldots \ldots \ldots \ldots \ldots \ldots \ldots \ldots$

Comptes de dépenses, b-5.5.1 . . . . . . . . . . . . . . . . 253

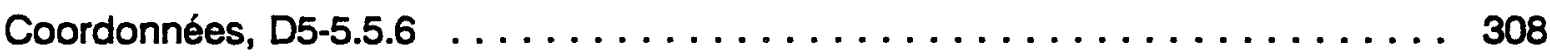

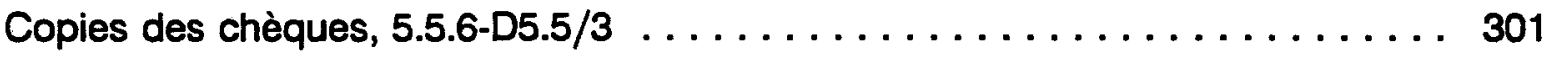

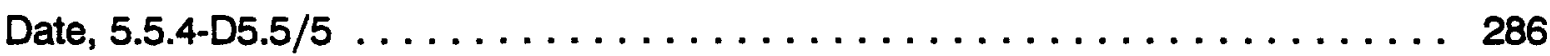

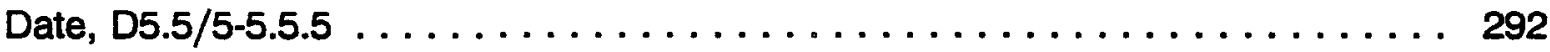

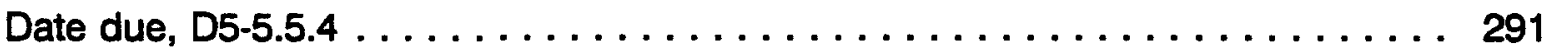

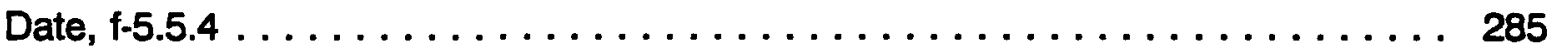

Dépenses, 5.5.3-D4 . . . . . . . . . . . . . . . . . . . . . 272

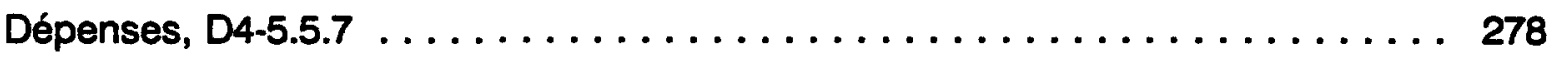

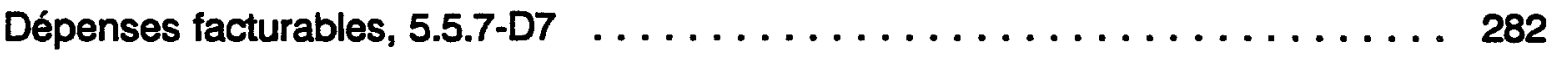

Dépenses non-facturables, $5.5 .7-D 10 \ldots \ldots \ldots \ldots \ldots \ldots$

Description du compte, $03-5.5 .3 \ldots \ldots \ldots \ldots \ldots \ldots \ldots$

Écritures de journal, $5.5 .3-D 8 \ldots \ldots \ldots \ldots \ldots \ldots \ldots$

Écritures de journal, $5.5 .6-\mathrm{D} 8 \ldots \ldots \ldots \ldots \ldots \ldots \ldots \ldots \ldots$

Écritures de journal, $5.5 .7-D 8 \ldots \ldots \ldots \ldots \ldots \ldots \ldots$

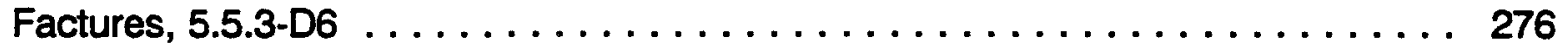

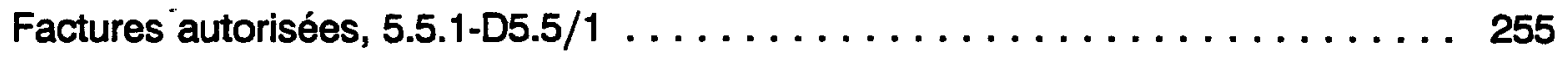

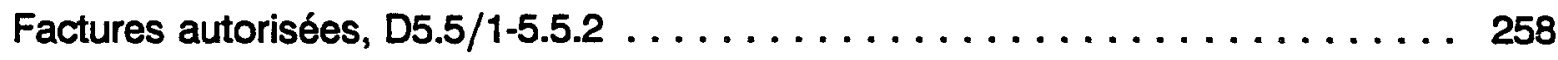




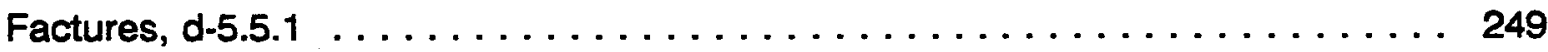

Factures, $06-5.5 .4 \ldots \ldots \ldots \ldots \ldots \ldots \ldots \ldots \ldots \ldots \ldots$

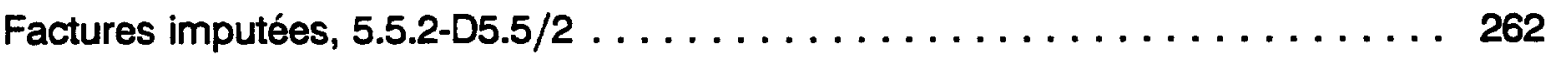

Factures imputées, D5.5/2-5.5.3 . . . . . . . . . . . . . . . 265

Factures payables, $06.5 .5 .6 \ldots \ldots \ldots \ldots \ldots \ldots$

Factures payées, $5.5 .5-D 5.5 / 3 \ldots \ldots \ldots \ldots \ldots \ldots \ldots$

Information fournisseurs, $5.5 .8-D 5 \ldots \ldots \ldots \ldots \ldots \ldots \ldots \ldots \ldots$

Information sur paiement, 5.5.6-D6 . . . . . . . . . . . . . . . 300

Liste des factures à payer, $5.5 .4-5.5 .5 \ldots \ldots \ldots \ldots \ldots$. . . . . . . . . . . 287

Montants, D5.5/6-5.5.6 . . . . . . . . . . . . . . . . . . . . . . . 299

Nom du fournisseur, $05-5.5 .3 \ldots \ldots \ldots \ldots \ldots \ldots \ldots$

Nom du fournisseur, $05-5.5 .4 \ldots \ldots \ldots \ldots \ldots \ldots \ldots$

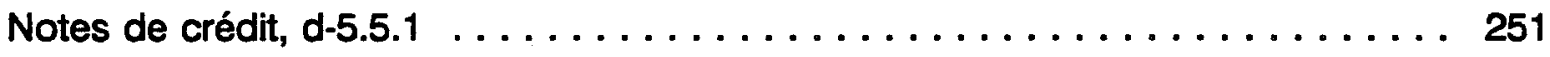

Numéro d'écritures, $D 9-5.5 .3 \ldots \ldots \ldots \ldots \ldots \ldots \ldots \ldots \ldots \ldots$

Numéro de compte, D3-5.5.2 . . . . . . . . . . . . . . . . 260

Numéro du client, D2-5.5.3 . . . . . . . . . . . . . . . . . . . . . 269

Paiements partiels ou retenus, $5.5 .5-D 6 \ldots \ldots \ldots \ldots$

\section{Processus 5.6, Produire les états finan-}

ciers

Diagramme de flux de données 
Processus de traitement $\ldots \ldots \ldots \ldots \ldots \ldots \ldots \ldots \ldots \ldots \ldots, 314$

Enregistrer les écritures automatiques, $5.6 .2 \ldots \ldots \ldots \ldots \ldots \ldots \ldots \ldots \ldots$

Produire auxiliaire des T.E.C., $5.6 .5 \ldots \ldots \ldots \ldots \ldots \ldots \ldots \ldots \ldots \ldots . \ldots \ldots$

Produire l'auxiliaire comptes à payer, $5.6 .7 \ldots \ldots \ldots \ldots \ldots \ldots \ldots \ldots \ldots$

Produire l'auxiliaire des C.A.R., $5.5 .6 \ldots \ldots \ldots \ldots \ldots \ldots \ldots \ldots \ldots \ldots, \ldots \ldots \ldots$

Produire le grand-livre, $5.6 .3 \ldots \ldots \ldots \ldots \ldots \ldots \ldots \ldots \ldots \ldots \ldots \ldots \ldots$

Produire le grand-livre cumulatif, $5.6 .4 \ldots \ldots \ldots \ldots \ldots \ldots \ldots \ldots \ldots \ldots \ldots$

Produire les états financiers, $5.6 .8 \ldots \ldots \ldots \ldots \ldots \ldots \ldots \ldots \ldots \ldots \ldots \ldots$

Saisir les régularisations, $5.6 .1 \ldots \ldots \ldots \ldots \ldots \ldots \ldots \ldots \ldots \ldots \ldots \ldots \ldots \ldots \ldots \ldots$

Flux de données $\ldots \ldots \ldots \ldots \ldots \ldots \ldots \ldots \ldots \ldots \ldots \ldots \ldots$

Auxiliaire des comptes à payer, $5.6 .7-g \ldots \ldots \ldots \ldots \ldots \ldots \ldots \ldots \ldots . \ldots . \ldots 369$

Auxiliaire des comptes a recevoir, $5.6 .5-\mathrm{g} \ldots \ldots \ldots \ldots \ldots \ldots \ldots \ldots \ldots 4$

Auxiliaire des travaux en cours, $5.6 .5-\mathrm{g} \ldots \ldots \ldots \ldots \ldots \ldots \ldots \ldots \ldots 35$

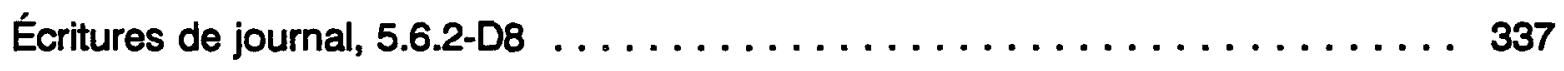

Écritures de journal, D5.6/1-5.6.2 $\ldots \ldots \ldots \ldots \ldots \ldots \ldots \ldots \ldots \ldots \ldots . \ldots \ldots$

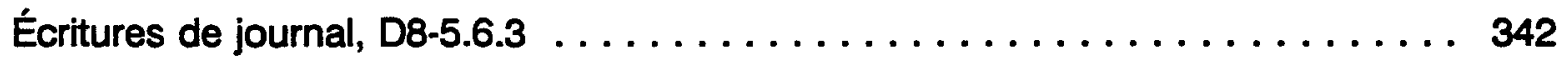

Écritures de journal, $D 8-5.6 .4 \ldots \ldots \ldots \ldots \ldots \ldots \ldots \ldots \ldots \ldots \ldots \ldots$

Écritures de journal, $D 8-5.6 .8 \ldots \ldots \ldots \ldots \ldots \ldots \ldots \ldots \ldots \ldots \ldots \ldots \ldots \ldots$

États financiers, $5.6 .8-g \ldots \ldots \ldots \ldots \ldots \ldots \ldots \ldots \ldots \ldots \ldots \ldots \ldots \ldots \ldots \ldots$

Grand-livre, $5.6 .3-g \ldots \ldots \ldots \ldots \ldots \ldots \ldots \ldots \ldots \ldots \ldots \ldots \ldots \ldots . \ldots \ldots$

Grand-livre cumulatif, $5.6 .4-g \quad \ldots \ldots \ldots \ldots \ldots \ldots \ldots \ldots \ldots \ldots \ldots \ldots \ldots$

Information sur les comptes, $012-5.6 .8 \ldots \ldots \ldots \ldots \ldots \ldots \ldots \ldots \ldots \ldots . \ldots . \ldots \ldots$

Nom des comptes, $012-5.6 .3 \ldots \ldots \ldots \ldots \ldots \ldots \ldots \ldots \ldots \ldots \ldots . \ldots . \ldots . \ldots . \ldots$ 


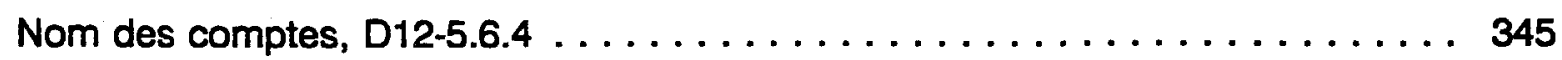

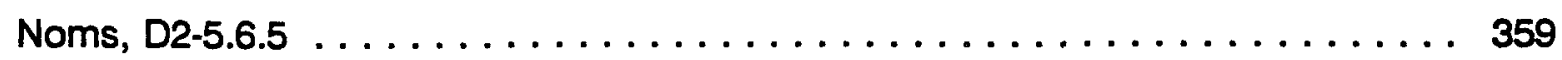

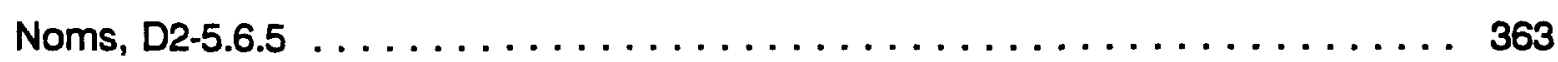

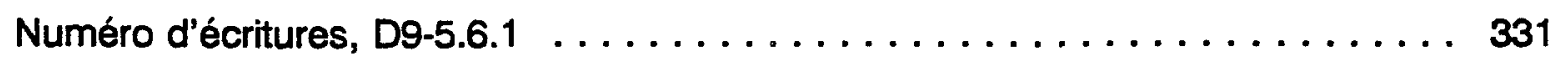

Numéro de comptes, $D 12-5.6 .1 \ldots \ldots \ldots \ldots \ldots \ldots \ldots \ldots \ldots \ldots \ldots \ldots \ldots$

Numéros de clients, D2-5.6.5 $\ldots \ldots \ldots \ldots \ldots \ldots \ldots \ldots \ldots \ldots \ldots \ldots$

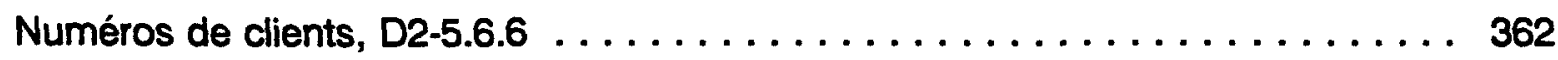

Numéros de comptes, D12-5.6.3 $\ldots \ldots \ldots \ldots \ldots \ldots \ldots \ldots \ldots \ldots \ldots .340$

Numéros de comptes, $012-5.6 .4 \ldots \ldots \ldots \ldots \ldots \ldots \ldots \ldots \ldots \ldots \ldots \ldots \ldots$

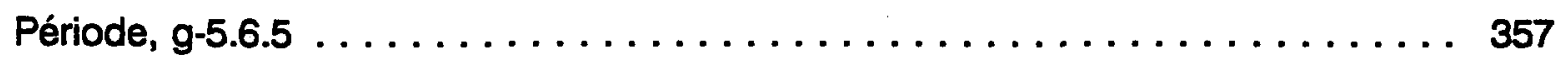

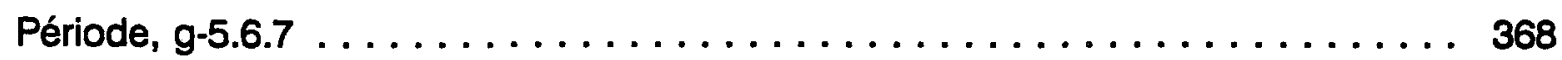

Régularisations, $5.6 .1-\mathrm{D} 8 \ldots \ldots \ldots \ldots \ldots \ldots \ldots \ldots \ldots \ldots \ldots \ldots \ldots \ldots \ldots \ldots$

Régularisations, g-5.6.1 $\ldots \ldots \ldots \ldots \ldots \ldots \ldots \ldots \ldots \ldots \ldots \ldots \ldots \ldots \ldots \ldots$

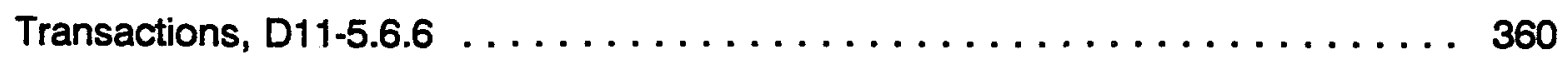

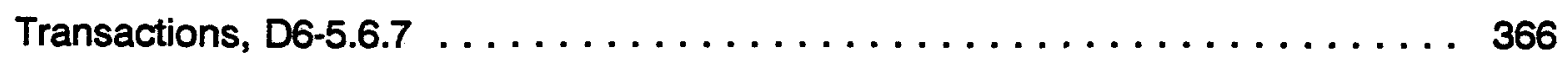

Transactions, $\mathbf{D} 7-5.6 .5 \ldots \ldots \ldots \ldots \ldots \ldots \ldots \ldots \ldots \ldots \ldots \ldots \ldots \ldots \ldots \ldots$ 